
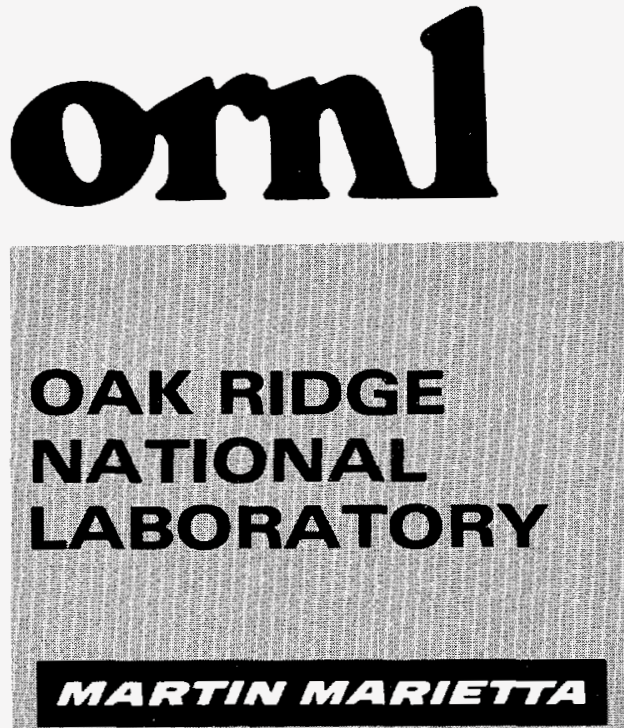

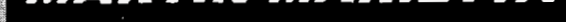
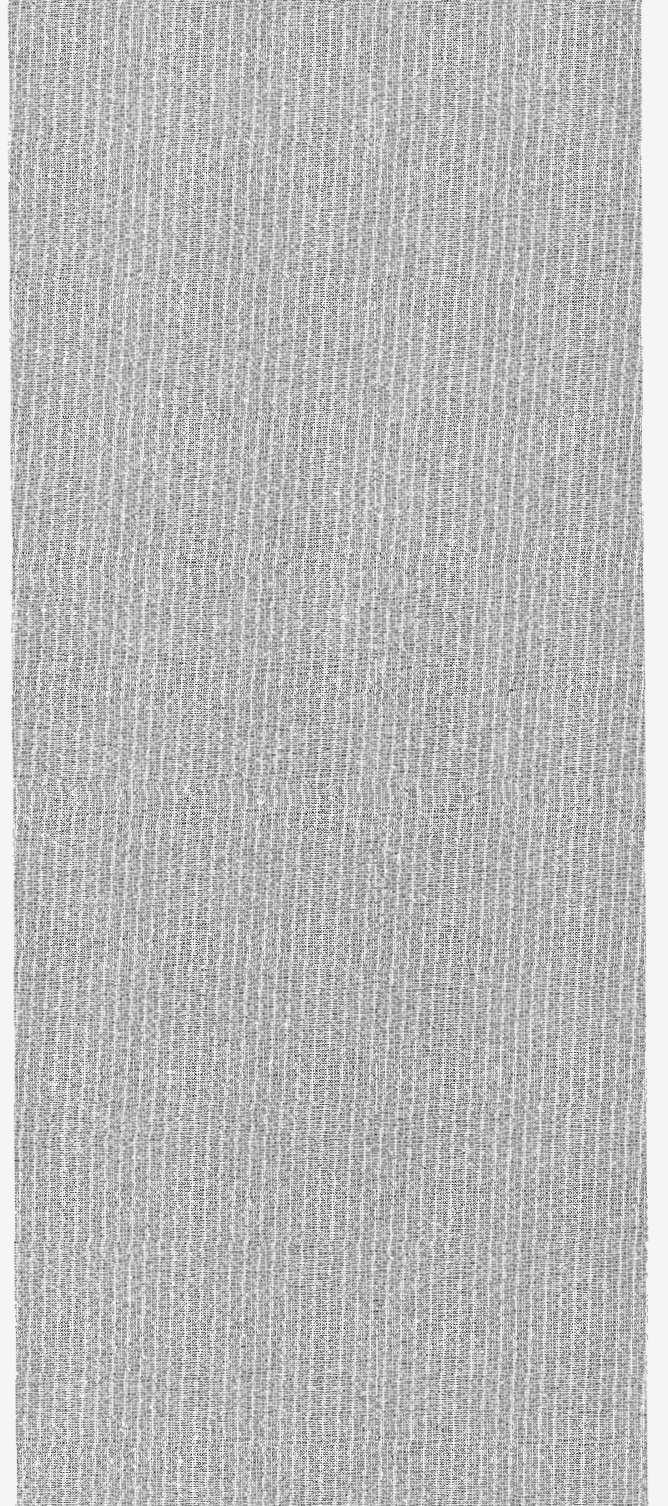

MARTIN MARIETTA ENERGY SYSTEMS, INC.

FOR THE UNITED STATES

OEPARTMENT OF ENEREY
ORNL/TM-10701

Dist. Category UC-535

\title{
Analysis of the JASPER Program Radial Shield Attenuation Experiment
}

C. O. Slater

\section{MASTER}


This report has been reproduced directly from the best available copy.

Available to DOE and DOE contractors from the Office of Scientific and Technical information, P.O. Box 62, Oak Ridge, TN 37831; prices available from (615) 576-8401, FTS 626-8401.

This report was prepared as an account of work sponsored by an agency of the United States Government. Neither the United States Government nor any agency thereof, nor any of their employees, makes any warranty, express or implied, or assumes any legal liability or responsibility for the accuracy, completeness, or usefulness of any information, apparatus, product, or process disclosed, or represents that its use would not infringe privately owned rights. Reference herein to any specific commercial product, process, or service by trade name, trademark, manufacturer, or otherwise, does not necessarily constitute or imply its endorsement, recommendation, or favoring by the United States Government or any agency thereof. The views and opinions of authors expressed herein do not necessarily state or reflect those of the United States Government or any agency thereof. 
ORNL/TM-10701

Dist. Category UC-535'

\title{
Engineering Physics and Mathematics
}

\section{Analysis of the JASPER Program Radial Shield Attenuation Experiment}

\author{
C. O. Slater
}

Date Published_January 1993

\section{DISCLAIMER}

\begin{abstract}
This report was prepared as an account of work sponsored by an agency of the United States Government. Neither the United States Government nor any agency thereof, nor any of their employees, makes any warranty, express or implied, or assumes any legal liability or responsibility for the accuracy, completeness, or usefulness of any information, apparatus, product, or process disclosed, or represents that its use would not infringe privately owned rights. Reference herein to any specific commercial product, process, or service by trade name, trademark, manufacturer, or otherwise does not necessarily constitute or imply its endorsement, recommendation, or favoring by the United States Government or any agency thereof. The views and opinions of authors expressed herein do not necessarily state or reflect those of the United States Government or any agency thereof.
\end{abstract}

Prepared by the

OAK RIDGE NATIONAL LABORATORY

Oak Ridge, Tennessee 37831

managed by

MARTIN MARIETTA ENERGY SYSTEMS, INC.

for the

U.S. DEPARTMENT OF ENERGY

under contract DE-AC05-84OR21400 


\section{Hatsons}




\section{DISCLAIMER}

Portions of this document may be illegible in electronic image products. Images are produced from the best available original document. 


\section{TABLE OF CONTENTS}

LIST OF FIGURES $\ldots \ldots \ldots \ldots \ldots \ldots \ldots \ldots \ldots \ldots \ldots \ldots \ldots$

LIST OF TABLES $\ldots \ldots \ldots \ldots \ldots \ldots \ldots \ldots \ldots \ldots \ldots \ldots \ldots \ldots \ldots \ldots$

ABSTRACT $\ldots \ldots \ldots \ldots \ldots \ldots \ldots \ldots \ldots \ldots \ldots \ldots \ldots \ldots \ldots \ldots \ldots \ldots$

ACKNOWLEDGMENTS $\ldots \ldots \ldots \ldots \ldots \ldots \ldots \ldots \ldots \ldots \ldots \ldots \ldots \ldots \ldots$

I. INTRODUCTION $\ldots \ldots \ldots \ldots \ldots \ldots \ldots \ldots \ldots \ldots \ldots \ldots \ldots \ldots \ldots \ldots$

2. EXPERIMENTAL DESIGN ......................

3. ANALYSIS OF THE EXPERIMENT $\ldots \ldots \ldots \ldots \ldots \ldots \ldots \ldots \ldots$

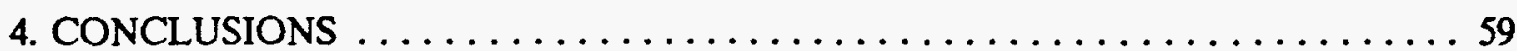

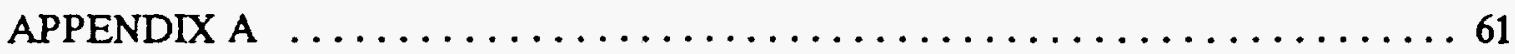

APPENDIX B $\ldots \ldots \ldots \ldots \ldots \ldots \ldots \ldots \ldots \ldots \ldots \ldots \ldots \ldots \ldots \ldots$

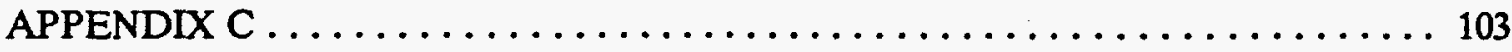

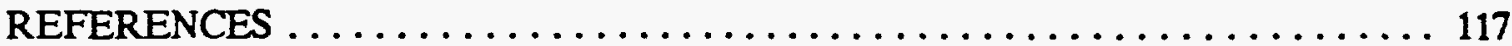





\section{LIST OF FIGURES}

$\underline{\text { Page }}$

Fig. 1. The Tower Shielding Reactor-II (TSR-II) with its wide-beam collimator . . . . 4

Fig. 2. Comparison of ENDF/B-V (Mat. 1160) and LLNL (Mat. 8811) "B total cross sections in the 61 -group structure $\ldots \ldots \ldots \ldots \ldots \ldots \ldots \ldots \ldots$

Fig. 3. Calculated versus measured $\mathrm{E}>0.05 \mathrm{MeV}$ neutron spectra on centerline $178.8 \mathrm{~cm}$ behind Configuration I.A . . . . . . . . . . . . . 13

Fig. 4. Calculated versus measured $\mathrm{E}>0.05 \mathrm{MeV}$ neutron spectra on centerline $47.3 \mathrm{~cm}$ behind Configuration II.D . . . . . . . . . . . . . . 14

Fig. 5. Calculated versus measured $\mathrm{E}>0.05 \mathrm{MeV}$ neutron spectra on centerline

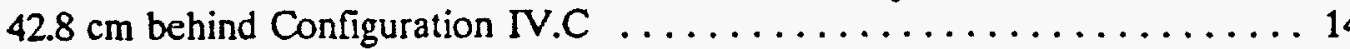

Fig. 6. Calculated versus measured $\mathrm{E}>0.05 \mathrm{MeV}$ neutron spectra on centerline $51.1 \mathrm{~cm}$ behind Configuration VI.A ................. 15

Fig. 7. Calculated versus measured $\mathrm{E}>0.05 \mathrm{MeV}$ neutron spectra on centerline $63.2 \mathrm{~cm}$ behind Configuration VI.F . . . . . . . . . . . . . 15

Fig. 8. Comparison of normalized calculated neutron spectra behind the two

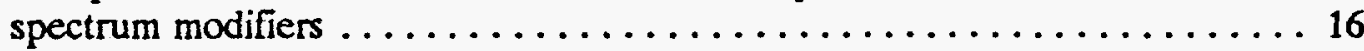

Fig. 9. Comparison of calculated and measured 5-in. Bonner ball count rates for a horizontal traverse $30 \mathrm{~cm}$ behind Configuration I.A (C/E range: 0.74-1.06) . . 33

Fig. 10. Comparison of calculated and measured 5 -in. Bonner ball count rates for a horizontal traverse $30 \mathrm{~cm}$ behind Configuration II.A (C/E range: 0.75-0.92) . 34

Fig. 11. Comparison of calculated and measured 5-in. Bonner ball count rates for a horizontal traverse $30 \mathrm{~cm}$ behind Configuration II.D (C/E range: 0.28-0.58) . 35

Fig. 12. Comparison of calculated and measured 5-in. Bonner ball count rates for a horizontal traverse $30 \mathrm{~cm}$ behind Configuration II.E (C/E range: 0.46-0.59) . . 36

Fig. 13. Comparison of calculated and measured 5-in. Bonner ball count rates for a horizontal traverse $30 \mathrm{~cm}$ behind Configuration III.A (C/E range: 0.96-1.09) . . 37

Fig. 14. Comparison of calculated and measured 5-in. Bonner ball count rates for a horizontal traverse $30 \mathrm{~cm}$ behind Configuration III.B (C/E range: 0.81-0.94) . 38

Fig. 15. Comparison of calculated and measured 5-in. Bonner ball count rates for a horizontal traverse $30 \mathrm{~cm}$ behind Configuration III.C (C/E range: 0.77-0.89) . 39 


\section{LIST OF FIGURES (Cont'd)}

Fig. 16. Comparison of calculated and measured 5 -in. Bonner ball count rates for a horizontal traverse $30 \mathrm{~cm}$ behind Configuration III.E (C/E range: $0.66-0.82$ ) . 40

Fig. 17. Comparison of calculated and measured 5-in. Bonner ball count rates for a horizontal traverse $30 \mathrm{~cm}$ behind Configuration IV.A (C/E range: 0.76-0.87) . 41

Fig. 18. Comparison of calculated and measured 5-in. Bonner ball count rates for a horizontal traverse $30 \mathrm{~cm}$ behind Configuration IV.C (C/E range: $0.71-0.95$ ) . 42

Fig. 19. Comparison of calculated and measured 5-in. Bonner ball count rates for a horizontal traverse $30 \mathrm{~cm}$ behind Configuration IV.G (C/E range: 0.32-0.61) . 43

Fig. 20. Comparison of calculated and measured 5-in. Bonner ball count rates for a horizontal traverse $30 \mathrm{~cm}$ behind Configuration V.A (C/E range: 0.93-1.08) . 44

Fig. 21. Comparison of calculated and measured 5-in. Bonner ball count rates for a horizontal traverse $30 \mathrm{~cm}$ behind Configuration V.B (C/E range: 0.90-1.02) .. 45

Fig. 22. Comparison of calculated and measured 5-in. Bonner ball count rates for a horizontal traverse $30 \mathrm{~cm}$ behind Configuration V.D (C/E range: 0.87-1.01) . 46

Fig. 23. Comparison of calculated and measured 5-in. Bonner ball count rates for a horizontal traverse $30 \mathrm{~cm}$ behind Configuration V.G (C/E range: 0.87-1.06) . 47

Fig. 24. Comparison of calculated and measured 5-in. Bonner ball count rates for a horizontal traverse $30 \mathrm{~cm}$ behind Configuration $\mathrm{VJ}(\mathrm{C} / \mathrm{E}$ range: 1.13-1.28) . . 48

Fig. 25. Comparison of calculated and measured 5-in. Bonner ball count rates for a borizontal traverse $30 \mathrm{~cm}$ behind Configuration VI.A (C/E range: 0.72-0.87) . 49

Fig. 26. Comparison of calculated and measured 5-in. Bonner ball count rates for a horizontal traverse $30 \mathrm{~cm}$ behind Configuration VI.B (C/E range: 0.79-0.89) . 50

Fig. 27. Comparison of calculated and measured 5-in. Bonner ball count rates for a horizontal traverse $30 \mathrm{~cm}$ behind Configuration VI.D (C/E range: 0.79-0.87) . 51

Fig. 28. Comparison of calculated and measured 5-in. Bonner ball count rates for a horizontal traverse $30 \mathrm{~cm}$ behind Configuration VI.F (C/E range: 0.80-0.89) . 52

Fig. 29. Comparison of calculated and measured 5-in. Bonner ball count rates for a horizontal traverse $30 \mathrm{~cm}$ behind Configuration VII.A (C/E range: 0.70-0.82) . 53

Fig. 30. Comparison of calculated and measured 5-in. Bonner ball count rates for a horizontal traverse $30 \mathrm{~cm}$ behind Configuration VII.B (C/E range: 0.65-0.76) . 54 


\section{LIST OF FIGURES (Cont'd)}

Fig. 31. Comparison of calculated and measured 5-in. Bonner ball count rates for a horizontal traverse $30 \mathrm{~cm}$ behind Configuration VII.C (C/E range: $0.56-0.73$ ) . 55

Fig. 32. Comparison of calculated 61-broad-group and 174-fine-group fast-neutron spectra with the measured spectrum on centerline $47.3 \mathrm{~cm}$ behind Configuration II.D $\ldots \ldots \ldots \ldots \ldots \ldots \ldots \ldots \ldots \ldots \ldots \ldots \ldots$

Fig. 33. Comparison of calculated 61-broad-group and 174-fine-group fast-neutron spectra with the measured spectrum on centerline $63.2 \mathrm{~cm}$ behind Configuration VI.F

Fig. B1. Sketch of the configuration for measurements under section I.A of the experimental program plan

Fig. B2. Sketch of the configuration for measurements under section V.A of the experimental program plan

Fig. B3. Sketch of the configuration for measurements under sections II.A-C,E and II.D (3-5) of the experimental program plan

Fig. B4. Sketch of the configuration for measurements under sections II.D (1-2) of the experimental program plan $\ldots \ldots \ldots \ldots \ldots \ldots \ldots \ldots \ldots$

Fig. B5. Sketches of the stainless steel containers used to form the $\mathrm{B}_{4} \mathrm{C}$ shield slabs $\ldots 84$

Fig. B6. Sketch of the configuration for measurements under sections III.A,C-E of the experimental program plan $\ldots \ldots \ldots \ldots \ldots \ldots \ldots \ldots \ldots$

Fig. B7. Sketch of the configuration for measurements under section III.B of the experimental program plan

Fig. B8. Sketch of the configuration for measurements under section IV.A of the

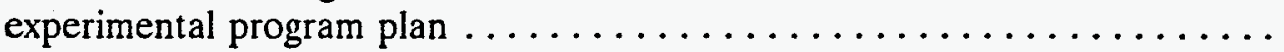

Fig. B9. Sketch of the configuration for measurements under section IV.B of the experimental program plan

Fig. B10. Sketch of the configuration for measurements under sections IV.C (1-2) of the experimental program plan $\ldots \ldots \ldots \ldots \ldots \ldots \ldots \ldots \ldots$

Fig. B11. Sketch of the configuration for measurements under sections IV.C (3-5) through IV.I of the experimental program plan 90 


\section{LIST OF FIGURES (Cont'd)}

Fig. B12. Sketch of the configuration for measurements under section IV.IA of the experimental program plan $\ldots \ldots \ldots \ldots \ldots \ldots \ldots \ldots \ldots \ldots \ldots \ldots \ldots \ldots$

Fig. B13. Sketch of the configuration for measurements under section IV.J of the experimental program plan $\ldots \ldots \ldots \ldots \ldots \ldots \ldots \ldots \ldots$

Fig. B14. Sketch of the configuration for measurements under section IV.K of the experimental program plan

Fig. B15. Sketch of the configuration for measurements under section IV.L of the experimental program plan $\ldots \ldots \ldots \ldots \ldots \ldots \ldots \ldots \ldots \ldots$

Fig. B16. Sketch of the configuration for measurements under section IV.M of the experimental program plan

Fig. B17. Sketch of the configuration for measurements under sections V.B-D of the

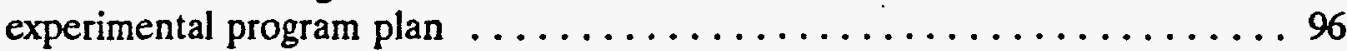

Fig. B18. Sketch of the configuration for measurements under sections V.E-G of the experimental program plan ..................... 97

Fig. B19. Sketch of the configuration for measurements under sections V.H-J of the experimental program plan $\ldots \ldots \ldots \ldots \ldots \ldots \ldots \ldots \ldots$

Fig. B20. Sketch of the configuration for measurements under sections VI.A (1-2) of the experimental program plan $\ldots \ldots \ldots \ldots \ldots \ldots \ldots \ldots . \ldots 99$

Fig. B21. Sketch of the configuration for measurements under sections VI.A (3-5) through VI.E and VI.F (3-5) of the experimental program plan . . . . . 100

Fig. B22. Sketch of the configuration for measurements under sections VI.F (1-2) of the experimental program plan .................... 101

Fig. B23. Sketch of the configuration for measurements under sections VII.A-D of the experimental program plan 


\section{LIST OF TABLES}

Page

Table 1. Atomic Densities $\left(\mathrm{b}^{-1} \cdot \mathrm{cm}^{-1}\right)$ for Constituents of Materials Used in the

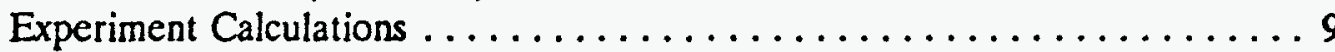

Table 2. Bulk Densities for $\mathrm{B}_{4} \mathrm{C}$ Powder in Slabs $\ldots \ldots \ldots \ldots \ldots \ldots \ldots \ldots \ldots$

Table 3. Comparison of Calculated and Measured Bonner Ball Count Rates $\left(\mathrm{s}^{-1} \cdot \mathrm{W}^{-1}\right)$ at the Spectrum Measurement Locations ............. 17

Table 4. Comparison of Calculated and Measured Bare $\mathrm{BF}_{3}$ Detector Count Rates $\left(\mathrm{s}^{-1} \cdot \mathrm{W}^{-1}\right)$ Close Behind Configurations . . . . . . . . . . . . . 19

Table 5. Comparison of Calculated and Measured Cd-Covered $\mathrm{BF}_{3}$ Count Rates

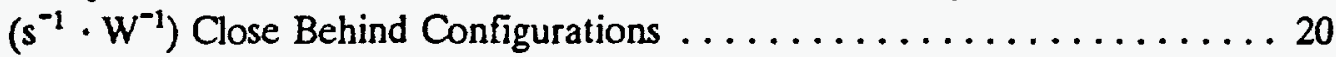

Table 6. Comparison of Calculated and Measured 3-in Bonner Ball Count Rates $\left(\mathrm{s}^{-1} . \mathrm{W}^{-1}\right)$ Close Behind Configurations . . . . . . . . . . . . . 21

Table 7. Comparison of Calculated and Measured 5-in. Bonner Ball Count Rates $\left(\mathrm{s}^{-1} \cdot \mathrm{W}^{-1}\right)$ Close Behind Configurations ..................... 22

Table 8. Comparison of Calculated and Measured 8-in. Bonner Ball Count Rates

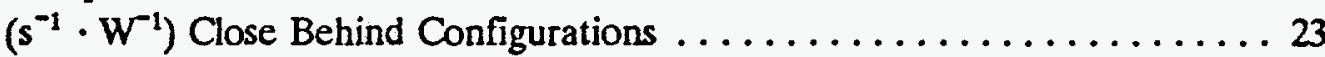

Table 9. Comparison of Calculated and Measured 10-in. Bonner Ball Count Rates $\left(\mathrm{s}^{-1} \cdot \mathrm{W}^{-1}\right)$ Close Behind Configurations ....................... 24

Table 10. Comparison of Calculated and Measured Bare $\mathrm{BF}_{3}$ Detector Count Rates $\left(\mathrm{s}^{-1} \cdot \mathrm{W}^{-1}\right)$ Behind Configurations at Large Distances from the TSR-II Core

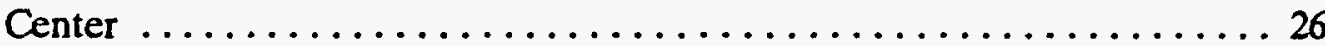

Table 11. Comparison of Calculated and Measured Cd-Covered $\mathrm{BF}_{3}$ Detector Count Rates $\left(\mathrm{s}^{-1} \cdot \mathrm{W}^{-1}\right)$ Behind Configurations at Large Distances from the TSR-II Core Center ................................ 27

Table 12. Comparison of Calculated and Measured 3-in. Bonner Ball Count Rates $\left(\mathrm{s}^{-1} \cdot \mathrm{W}^{-1}\right)$ Behind Configurations at Large Distances from the TSR-II Core Center ................................... 28

Table 13. Comparison of Calculated and Measured 5-in. Bonner Ball Count Rates $\left(\mathrm{s}^{-1} \cdot \mathrm{W}^{-1}\right)$ Behind Configurations at Large Distances from the TSR-II Core Center 


\section{LIST OF TABLES (Cont'd)}

$\underline{\text { Page }}$

Table 14. Comparison of Calculated and Measured 8-in. Bonner Ball Count Rates $\left(\mathrm{s}^{-1} \cdot \mathrm{W}^{-1}\right)$ Behind Configurations at Large Distances from the TSR-II

Core Center . . . . . . . . . . . . . . . . . . . 30

Table 15. Comparison of Calculated and Measured 10-in. Bonner Ball Count Rates $\left(\mathrm{s}^{-1} \cdot \mathrm{W}^{-1}\right)$ Behind Configurations at Large Distances from the TSR-II

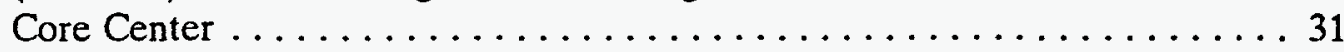

Table 16. Comparison of Calculated and Measured Bonner Ball Count Rates $\left(\mathrm{s}^{-1} \cdot \mathrm{W}^{-1}\right)$ in the Shielded Void Region of Several Configurations IV . . . 32

Table 17. Sensitivities of Neutron Fluxes to Neutron Cross Sections of Various Elements Comprising the Shields of Configuration II.D (1-2) . . . . . . 58

Table 18. Sensitivities of Neutron Fluxes to Neutron Cross Sections of Various Elements Comprising the Shields of Configuration IV.H $\ldots \ldots \ldots \ldots \ldots \ldots$

Table C1. 51-Group Response Function for the Bare $\mathrm{BF}_{3}$ Detector. Units are: (Counts $\cdot \mathrm{cm}^{2} /$ neutron) . . . . . . . . . . . . 104

Table C2. 51-Group Response Function for the Cd-Covered $\mathrm{BF}_{3}$ Detector.

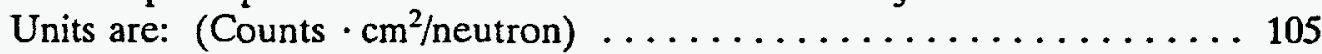

Table C3. 51-Group Response Function for the 3-inch Bonner Ball.

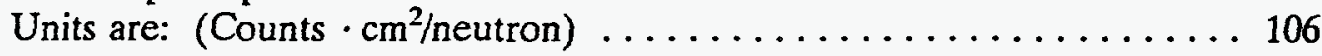

Table C4. 51-Group Response Function for the 5-inch Bonner Ball. Units are: (Counts $\cdot \mathrm{cm}^{2} /$ neutron) $\ldots \ldots \ldots \ldots \ldots \ldots \ldots \ldots$

Table C5. 51-Group Response Function for the 8-inch Bonner Ball. Units are: (Counts $\cdot \mathrm{cm}^{2} /$ neutron) 108

Table C6. 51-Group Response Function for the 10-inch Bonner Ball. Units are: (Counts $\cdot \mathrm{cm}^{2} /$ neutron) 109

Table C7. 61-Group Response Function for the Bare $\mathrm{BF}_{3}$ Detector.

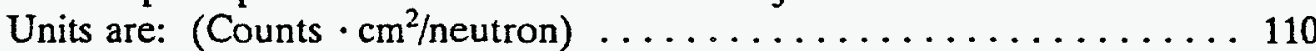

Table C8. 61-Group Response Function for the Cd-Covered $\mathrm{BF}_{3}$ Detector. Units are: (Counts $\mathrm{cm}^{2}$ /neutron) $\ldots \ldots \ldots \ldots \ldots \ldots \ldots \ldots \ldots \ldots \ldots$

Table C9. 61-Group Response Function for the 3-inch Bonner Ball. Units are: (Counts $\cdot \mathrm{cm}^{2} /$ neutron) 


\section{LIST OF TABLES (Cont'd)}

Table C10. 61-Group Response Function for the 5-inch Bonner Ball. Units are: (Counts $\cdot \mathrm{cm}^{2} /$ neutron) $\ldots \ldots \ldots \ldots \ldots \ldots \ldots \ldots \ldots \ldots \ldots$

Table C11. 61-Group Response Function for the 8-inch Bonner Ball.

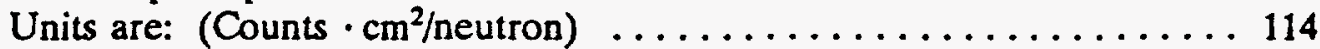

Table C12. 61-Group Response Function for the 10-inch Bonner Ball.

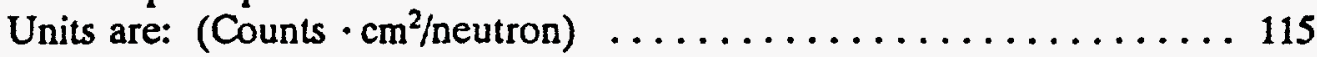




\begin{abstract}
The results of the analysis of the JASPER Program Radial Shield Attenuation Experiment are presented. The experiment was performed in 1986 at the ORNL Tower Shielding Facility. It is the first of six experiments in this cooperative Japanese and American program in support of shielding designs for advanced sodium-cooled reactors. Six different shielding configurations and subconfigurations thereof were studied. The configurations were calculated with the DOT-IV two-dimensional discrete ordinates radiation transport computer code using the $R-Z$ geometry option, a symmetric $S_{12}$ quadrature ( 96 directions), and cross sections from ENDF/B versions $I V$ and $V$ in either a 51- or 61-group structure. Auxiliary codes were used to compute detector responses and prepare cross sections and source input for the DOT-IV calculations. Calculated detector responses were compared with measured responses and the agreement was good to excellent in many cases. However, the agreement for configurations having thick steel or $\mathrm{B}_{4} \mathrm{C}$ regions or for some very large configurations was fair to poor. The disagreement was attributed to cross-section data, broad-group structure, or high background in the measurements. In particular, it is shown that two cross-section sets for ${ }^{11} \mathrm{~B}$ give very different results for neutron transmission through the thick $B_{4} C$ regions used in one set of experimental configurations. Implications for design calculations are given.
\end{abstract}




\section{ACKNOWLEDGMENTS}

The author greatly appreciates the discussions with D. T. Ingersoll, W. W. Engle, Jr., and F. J. Muckenthaler during the analysis of this experiment. The reviewers (Larry Williams and Mark Smith) are thanked for their valuable services. The program direction of D. T. Ingersoll and W. W. Engle, Jr. is appreciated. Due to a shutdown of the JASPER Program for a 3-year period at the TSF, this report has been in production for several years. The author thanks A. C. Alford and Gail Marvin for their earlier work in preparing the manuscript and C. R. Householder for her work in completing the final manuscript. 


\subsection{INTRODUCTION}

The shielding of components within liquid metal fast breeder reactor (LMFBR) designs is usually accomplished with combinations of stainless steel, boron carbide $\left(\mathrm{B}_{4} \mathrm{C}\right)$, and graphite, together with any thick sodium regions between the core and the component. Calculated radiation levels at those components can be quite uncertain due to: (1) the large number of mean-free-paths through which the radiation must be transported (deep penetration), (2) the three-dimensional complexities of the calculational geometry, and (3) the uncertainties associated with the methods and data used to calculate the radiation levels. A joint experimental program between the United States Department of Energy and the Japanese Power Reactor and Nuclear Fuel Development Corporation (PNC) was designed to address those concerns. The Japanese-American Shielding Program of Experimental Research (JASPER) consists of six experiments which would provide data that could be used to design LMFBR shields or verify calculational methods and data used to calculate radiation transport through those shields. Reported here are the results of the analysis of the first of the JASPER experiments, the Radial Shield Attenuation Experiment, which was performed in 1986 at the ORNL Tower Shielding Facility. ${ }^{1}$ 


\subsection{THE EXPERIMENTAL DESIGN}

The experiment consisted of slab mockups of six proposed LMFBR shield designs and sub-mockups thereof placed after a spectrum modifier that was positioned in front of the Tower Shielding Reactor, a schematic drawing of which is shown in Fig. 1. The sub-mockups were included to isolate the shielding effect of various portions of the shield and to provide checkpoints for the calculations. In this report, the configurations are identified by the section numbers in the Experimental Program Plan found in Appendix A. The configurations are described briefly.

Two spectrum modifiers were used. The first of the spectrum modifiers (SM1) is shown in Fig. B1. It consists of slabs of carbon steel $(10 \mathrm{~cm})$, aluminum $(9 \mathrm{~cm})$, and boral $(2.5 \mathrm{~cm})$ followed by two $10.1-\mathrm{cm}$-thick natural $\mathrm{UO}_{2}$ blankets. This is Configuration I.A of the experimental program plan. It is used for all except one set of configurations. The second of the spectrum modifiers (SM2) is shown in Fig. B2. It consists of carbon steel $(10 \mathrm{~cm})$, aluminum $(9 \mathrm{~cm})$, boral $(2.5 \mathrm{~cm})$, and sodium $(183 \mathrm{~cm}$ plus $7.62 \mathrm{~cm}$ of aluminum in the walls of the six tanks) and is designated Configuration V.A in the experimental program plan. It is used only for configurations under section $\mathrm{V}$ of the plan.

Configurations II are shown in Figs. B3 and B4. These configurations represent near-core shields containing stainless steel and $\mathrm{B}_{4} \mathrm{C}$, with a thick $\mathrm{B}_{4} \mathrm{C}$ region following a moderately thick stainless steel region. The five $\mathrm{B}_{4} \mathrm{C}$ slabs shown in Fig. B5 were used to create the three $15.2-\mathrm{cm}$-thick shield segments called for in the plan. Note that the experimental program plan gives nominal thicknesses for the slab combinations, while the figures show the actual thicknesses. Fig. B4 shows an arrangement required to measure the neutron spectrum behind configuration II.D. Because of a high gamma-ray field, 7.62 $\mathrm{cm}$ of lead had to be used behind the configuration in order to obtain good spectrum data. Other measurements were made without the lead slabs; therefore, there are two Configurations II.D, each being further defined by the measurement subsections pertaining to them [e.g. II.D $(1,2)$ and II.D(3-5)]. The same applies to the other configurations for which lead was added in order to measure the neutron spectrum. Note also that the aluminum slabs in the shield mockups represent sodium-filled regions between shield components. The slabs are thinner than the sodium-filled regions they represent because aluminum is the better neutron attenuator.

Configurations III are shown in Figs. B6 and B7. These configurations are called benchmark and consist of stainless steel or stainless steel and $\mathrm{B}_{4} \mathrm{C}$ slabs following SM1.

Configurations IV are shown in Figs. B8-B16. These configurations represent the shield for a pool-type LMFBR and they consist of slabs of stainless steel, graphite, and $\mathrm{B}_{4} \mathrm{C}$ all following SM1. Many of these shields were so optically thick that radiation coming around the shield mockup created a large background problem for the measurements. Thus, beginning at section IV.I of the program plan, the plan was altered so that measurements would be made in a completely enclosed and shielded void region to reduce background interference (see Figs. B13-B16).

Configurations V are shown in Figs. B2 and B17-B19. They consist of SM2 and slabs of stainless steel, $B_{4} C$, and sodium and represent the shield for the intermediate heat exchanger in a pool-type LMFBR. The thicknesses shown for the sodium tanks are centerline measurements. The tanks are nominally $31.75 \mathrm{~cm}$ thick with $0.635-\mathrm{cm}$ end wall 


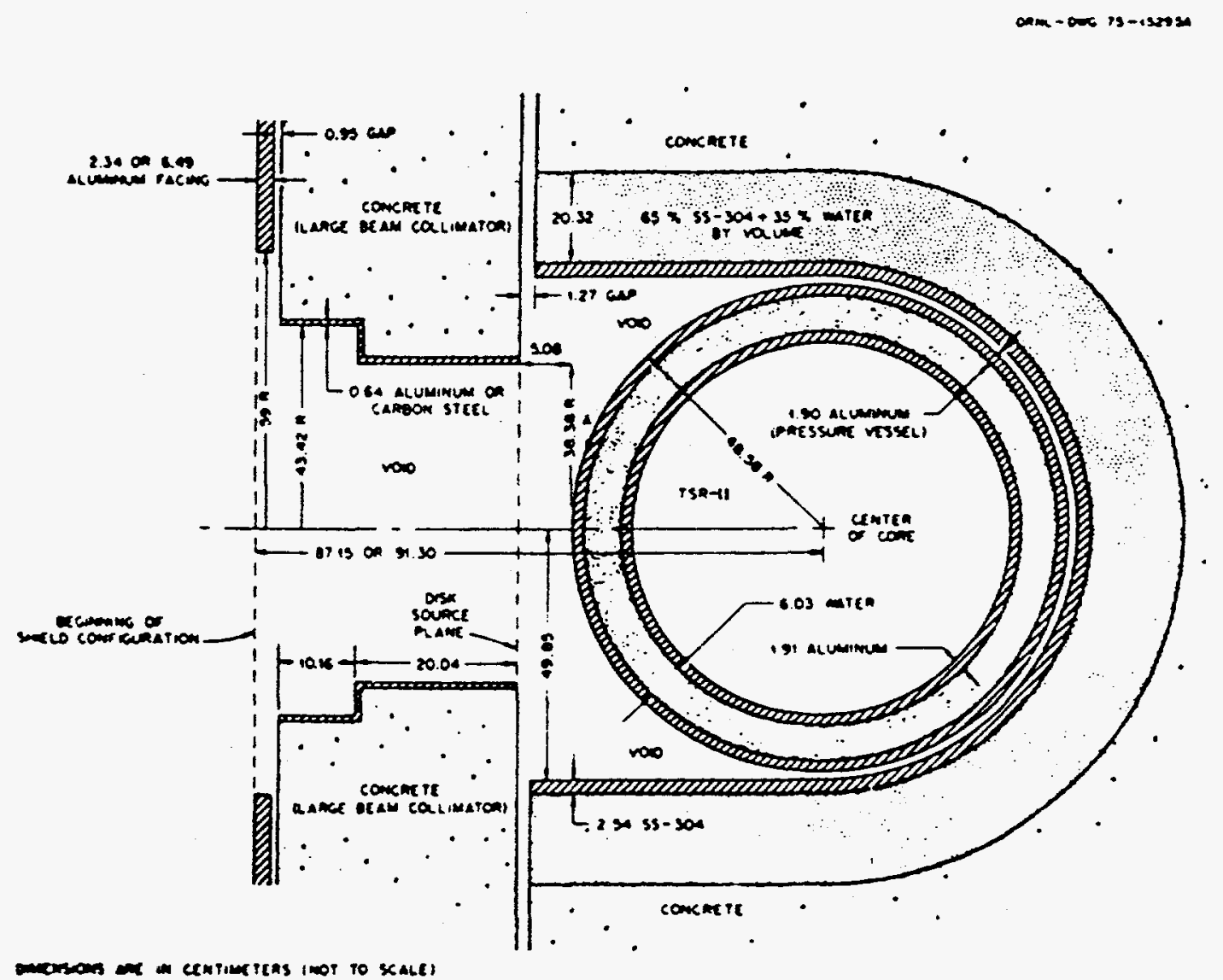

Fig. 1. The Tower Shielding Reactor-II (TSR-II) with its wide-beam collimator. 
and $1.27-\mathrm{cm}$ side wall thicknesses. Bulging, sagging, and bending of the tanks causes the wide variations in the thicknesses shown in the figures.

Configurations VI are shown in Figs. B20-B22 and are called the graphite benchmark. The configurations consist of SM1 followed by about $15 \mathrm{~cm}$ of stainless steel and various thicknesses of graphite. The two spectrum measurements required either lead alone or $\mathrm{B}_{4} \mathrm{C}$ and lead to reduce the gamma-ray background.

Finally, Configurations VII are shown in Fig. B23. Like Configurations II, these also represent a near-core shield. However, the shield represented is one having a thick region of stainless steel followed by a moderately thick $\mathrm{B}_{4} \mathrm{C}$ region and sodium. The mockups are preceded by SM1.

As the program plan shows (Appendix A), measurements were made with the bare and $\mathrm{Cd}$-covered $\mathrm{BF}_{3}$ detectors, the 3-, 5-, 8-, and 10-in Bonner balls in situ, $30 \mathrm{~cm}$ behind configurations, at locations where spectra were measured (3-, 4-, 5-, and 10-in balls only), and behind configurations at large distances from the reactor center. The Bonner balls consist of cadmium-covered spherical shells of polyethylene around a 5.08-cm-diameter spherical $\mathrm{BF}_{3}$ chamber. Note that the bare and cd-covered $\mathrm{BF}_{3}$ detectors are special cases of the Bonner balls having no polyethylene around them. Measurements were made with the $\mathrm{PNC}{ }^{3} \mathrm{He}$ detectors at the Bonner ball positions described above. Spectra were measured behind five configurations (I.A, II.D, IV.C, VI.A, and VI.F) with the NE-213 and Benjamin (hydrogen counter) spectrometers. The NE-213 spectrometer covers an energy range of 0.8 to $15.0 \mathrm{MeV}$ and the three hydrogen counters $(1,3$, and 10 atmospheres) cover the energy range $50 \mathrm{keV}$ to $1.5 \mathrm{MeV}$. 



\subsection{ANALYSIS OF THE EXPERIMENT}

\subsection{Analysis Methods and Input Data}

\subsubsection{Calculational Methods}

Calculations of neutron transport through the configurations were performed mainly with the DOT-IV ${ }^{2}$ two-dimensional discrete ordinates radiation transport computer code using the R-Z geometry option. (For the Cray computer, on which some of the calculations were performed, "DORT" is the analogous code.) DOT-IV was also used to calculate neutron fluxes in a limited void region behind the configurations when that void was not included in the geometry mockup for the configuration. The void region was one large enough to contain all the detector positions close behind the configurations (i.e., the $30-\mathrm{cm}$ centerline and traverse measurements, other close-in measurements for Configurations IV, and all spectrum measurement positions except that for Configuration I.A). Diagnostic and parameter-study cases were run using the ANISN ${ }^{3}$ one-dimensional discrete ordinates radiation transport code with the spherical geometry option. This code was used to check the effects of group structure, cross-section sets or versions, and material densities on calculated detector responses. It was also used to generate the forward and adjoint fluxes used in the limited sensitivity calculations which were performed with the SWANLAKE ${ }^{4}$ code.

Calculated detector responses were obtained in one of three ways. First, the measured fast-neutron spectra were assumed to be point values; therefore, for comparisons with measured values, the DOT-IV-calculated fluxes were used without modification. Second, Bonner ball count rates close behind the configurations were obtained using the DOTWRTPN* computer code (called DTWTPN on the Cray computer). This code calculates detector count rates by averaging DOT-IV point detector count rates over the reactor-side hemisphere of the Bonner balls. Third, Bonner ball count rates at detectors large distances behind the configurations were obtained using the DISKTRAN* computer code (called DSKTRN on the Cray computer). DISKTRAN uses the DOT-IV angular fluxes from the top or bottom of a cylinder to calculate neutron fluxes at points in a void region outside the cylinder and folds those fluxes with detector response functions to give calculated detector count rates. Because the Bonner balls are not point detectors, the detector locations were adjusted forward of the ball centers to the "centers of detection"s for each of the balls. DISKTRAN was also used to calculate the neutron fluxes at the spectrum location behind Configuration I.A.

The DOT-IV calculations used boundary fluxes as sources and most of the time the fluxes required some processing in order to be used in a particular calculation. The boundary fluxes were processed by the BSPRP2* and VFL2BDRY* (called VFL2BD on the Cray computer) computer codes. The code BSPRP2 processes DOT-IV boundary flux files for input to subsequent DOT-IV calculations. The flux files may be altered to (1) select specific boundary fluxes from a multiple boundary flux file, (2) create a source for a different spatial mesh or quadrature, (3) normalize the source or correct it for

* Computer codes written by the author. 
streaming effects, or (4) combine several of the above features. The code VFL2BDRY reads top and bottom boundary fluxes from a DOT-IV R-Z flux and moments file and creates a top or bottom boundary source file for a subsequent DOT-IV calculation. While scale factors by group and interval can be applied to the boundary fluxes, no mesh or quadrature change is allowed. One may, however, follow up with BSPRP2 if such changes are desired. VFL2BDRY was used in this experiment to prepare boundary sources for follow-on DOT-IV calculations of neutron fluxes in the void region behind configurations, although some of the same sources could have been used as internal boundary sources for bootstrapped calculations of some of the larger configurations. When a change was made in the group structure used in the analysis, the previously generated boundary sources were converted to the new group structure using the Cray computer code CHBSGP written by the author. The code uses lethargy weighting to calculate boundary fluxes for the new group structure.

A final code used, TSFDISKS, is explained is Sect. 3.1.4.

\subsubsection{Material Compositions}

The material compositions used in the calculations are based on the material analyses given in Ref. 1 or data from reports on the analysis of previous Tower Shielding Facility (TSF) experiments. The atom densities of the various elements comprising the materials are shown in Table 1 . The table lists the $\mathrm{B}_{4} \mathrm{C}$ atom densities for a material density of $1.0 \mathrm{~g} / \mathrm{cm}^{3}$. Table 2 shows the material densities for the five $\mathrm{B}_{4} \mathrm{C}$ slabs used in the experimental mockups. Mixtures for the slabs were formed by multplying the $\mathrm{B}_{4} \mathrm{C}$ mixture in Table 1 by the appropriate material density in Table 2.

\subsubsection{Cross Sections and Detector Response Data}

Initial calculations were performed with a 51-neutron-group structure using a crosssection library created in several steps for LMFBR reactor physics and shielding analyses. The data were mainly from ENDF/B-IV and were adequate for calculating some of the configurations as well as previous TSF experiments. However, the calculations showed very large underpredictions for Configurations II, which contained large amounts of $\mathrm{B}_{4} \mathrm{C}$ and stainless steel. A study of various cross section sets led to data sets for Fe and ${ }^{11} \mathrm{~B}$ that gave results superior to any obtained with the old data or with data from libraries having other group structures. The important differences in the two ${ }^{11} \mathrm{~B}$ cross-section sets occur mainly above $0.4 \mathrm{MeV}$ as the plot of the total cross section in Fig. 2 shows. The deeper cross-section window for the energy range $0.5-1.0 \mathrm{MeV}$ and the lower cross section around $2 \mathrm{keV}$ probably are responsible for the significantly increased neutron transmission through the $\mathrm{B}_{4} \mathrm{C}$ slabs when using the newer ${ }^{11} \mathrm{~B}$ cross-section data. Because the new cross section data were available for a group structure different from that used in the original calculations, the group structure for the calculations was then changed to a 61 neutron-group structure for which a new cross-section library had been created. ${ }^{6}$ Cross section data for Fe was obtained from a separate file created by $\mathrm{C}$. Y. Fu. ${ }^{7}$ This new $\mathrm{Fe}$ data has improved inelastic cross sections and generally gives more high-energy neutron transmission. 
Table 1. Atomic densities $\left(\mathrm{b}^{-1} \cdot \mathrm{cm}^{-1}\right)$ for constituents of materials used in the experiment calculations

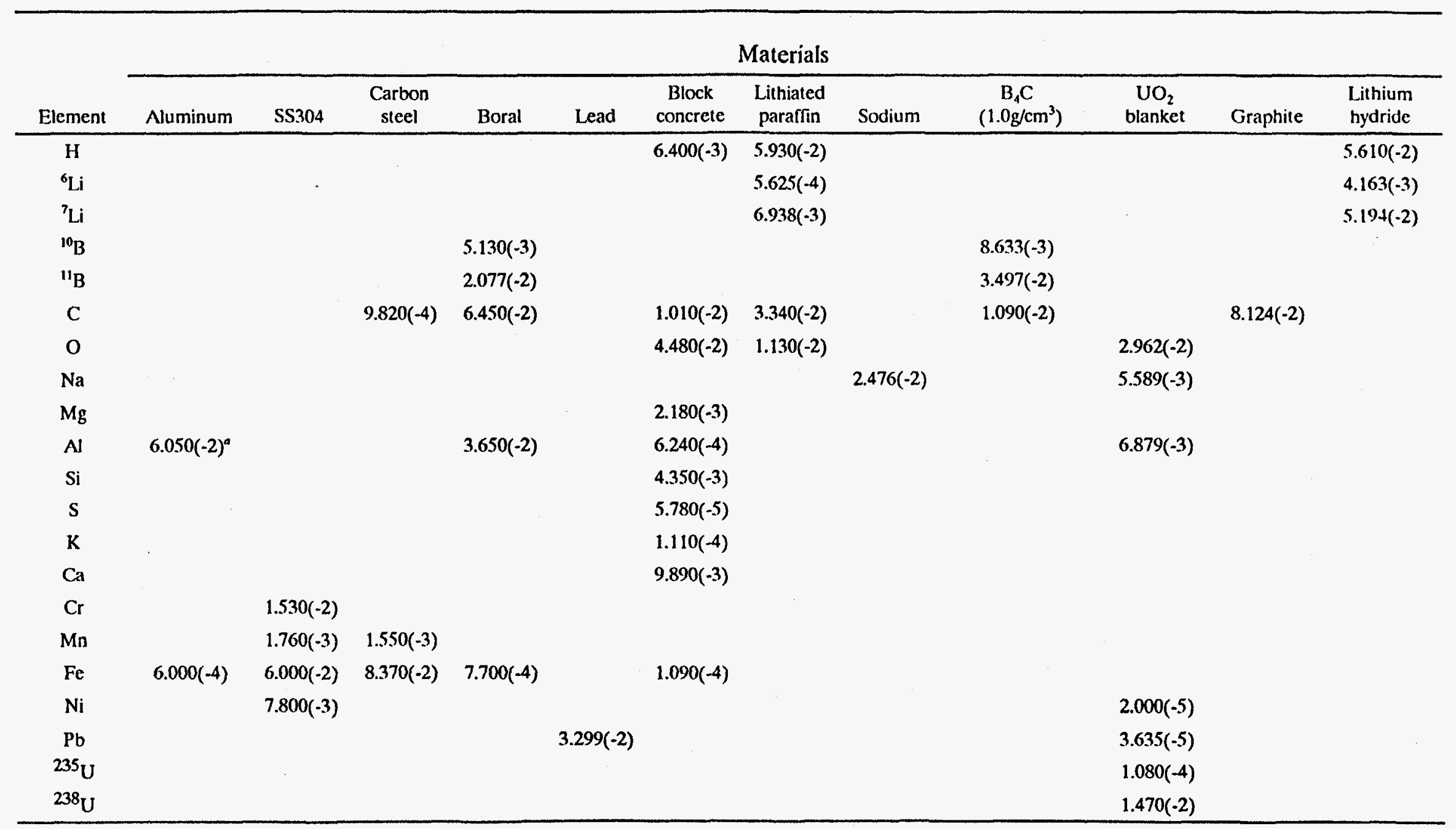

${ }^{a}$ Read as $6.050 \times 10^{-2}$. 
Table 2. Bulk densities for $B_{4} C$ powder in slabs.

\begin{tabular}{c|c}
\hline Slab designation & Density $\left(\mathrm{g} / \mathrm{cm}^{3}\right)$ \\
\hline $1 W$ & 1.42 \\
$2 W$ & 1.39 \\
$3 W$ & 1.37 \\
$4 W$ & 1.41 \\
$5 W$ & 1.44 \\
\hline
\end{tabular}

Bonner ball response functions in the 51 group structure were collapsed from Maerker's 100 -group data. ${ }^{8}$ These data are shown along with the group energy boundaries in Tables C1-C6. Data for the 61-group-structure were obtained from the 51-group-data using the AMP code. ${ }^{9}$ These data are shown in Tables $\mathrm{C} 7-\mathrm{C} 12$ along with the group energy boundaries.

\subsubsection{Reactor Source Calculation}

The source for the experiment was provided by the Tower Shielding Reactor II (TSR-II) with its wide-beam configuration as illustrated in Fig. 1. Maerker ${ }^{10}$ calculated the absolute neutron source on the surface of the TSR-II and presented the results as one-dimensional (1-D) angular fluxes for the eight outward angles of an $S_{16}$ quadrature set and for the 51-group structure. The 1-D angular fluxes were projected onto a symmetric $S_{12}$ two-dimensional (2-D) quadrature ( 96 directions) on side and end cylindrical surfaces surrounding the core to form an equivalent internal boundary source for a DOT-IV calculation of neutron transport from the reactor through its collimator and onto the spectrum modifier. The projection of the source from the 1-D to the 2-D geometry was performed with the TSFDISKS code written by the author. The DOT-IV calculation was performed on a geometry model containing the TSR-II, its collimator, and the iron, aluminum and boral portion of the spectrum modifiers (Figs. B1 and B2) and boundary fluxes were saved at the interface between the reactor collimator and the spectrum modifier. ${ }^{11}$ The output boundary fluxes were then used as the source for Configurations I.A, I.A, III.A-C, V.A, and VI.A. Boundary fluxes output from some of these calculations were then used as sources for larger configurations of which they are subsets. Configurations IV and VII at the outset used boundary fluxes from Configuration VI.A since they are identical out to the stainless steel slabs beyond the $\mathrm{UO}_{2}$ blankets. The source, which is located on the surface of the aluminum beyond the blankets, should not be affected by shield mockups beyond the stainless steel slabs. Internal boundary fluxes output from two axial locations in Configuration IV.J were used to calculate smaller and larger Configurations IV in order to reduce calculational effort. Likewise, boundary fluxes at two locations in Configuration V.A were used to calculate larger Configurations V. 


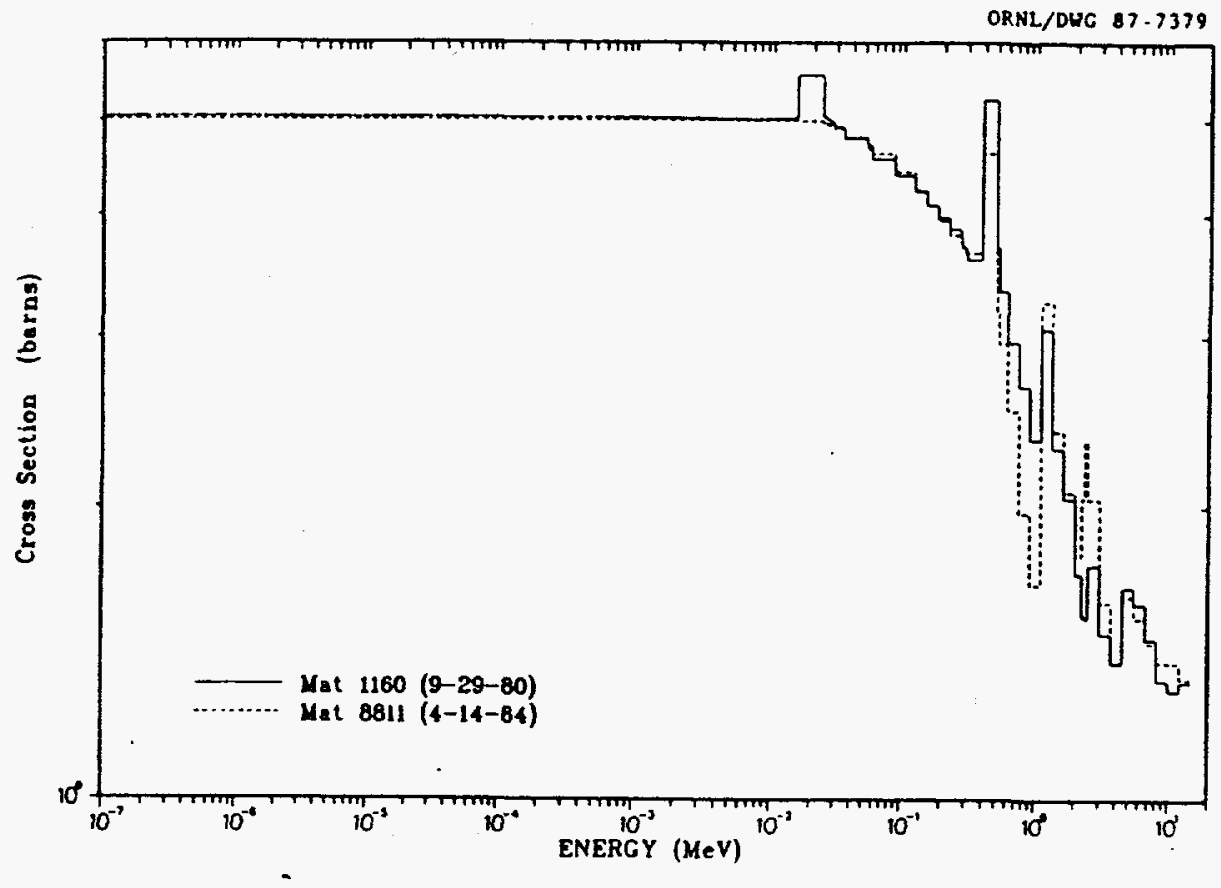

Fig. 2. Comparison of ENDF/B-V (Mat. 1160) and LLNL (Mat. 8811) ${ }^{11} \mathrm{~B}$ total cross sections in the 61-group structure. 


\subsection{Comparisons of Calculated and Measured Results}

In comparing the calculated and measured results, qualitative judgments are made as to the agrecment between the calculated and measured results. First of all, the results are generally compared according to the ratio of the calculated to the measured result. Since the quoted uncertainty for the Bonner ball measurements is $\pm 10 \%$, agreement is said to be excellent when calculation-to-experiment ratio ratios ( $C / E$ 's) lie between 0.9 and 1.1. For this analysis, agreement is good for $C / E$ 's between 0.8 and 0.9 or 1.1 and 1.2; fair for $\mathrm{C} / \mathrm{E}$ 's between 0.6 and 0.8 or 1.2 and 1.4 ; and poor for $\mathrm{C} / \mathrm{E}$ 's less than 0.6 or greater than 1.4 .

\subsubsection{Neutron Spectra Behind Configurations}

Neutron spectra were measured behind five configurations: I.A, II.D, IV.C, VI.A, and VI.F. For all except Configuration I.A, lead or $B_{4} C$ and lead were used to reduce gamma-ray background in order to obtain good spectrum data. For Configuration I.A the spectrum was measured a great distance behind the configuration to reduce the gamma-ray interference. Calculated and measured $\mathrm{E}>0.05 \mathrm{MeV}$ neutron spectra are compared in Figs. 3-7. The calculated results are in good agreement with the measured results, with the integrals of the calculated spectra being $11-16 \%$ smaller than the integrals of the measured spectra. However, the calculation-to-experiment ratio $(\mathrm{C} / \mathrm{E})$ for Configuration II.D appears to better than the plot indicates, since the calculated spectrum is significantly above measured in the 3 to $10 \mathrm{MeV}$ range and is below measured below $0.8 \mathrm{MeV}$. The compensating effects of the higher and lower fluxes probably gives Configuration II.D a higher $\mathrm{C} / \mathrm{E}$ than that for some of the configurations where the shapes of the calculated and measured flux curves agree better. While the newer ${ }^{11} \mathrm{~B}$ cross-section set causes a great improvement in the $C / E$ for Configuration II.D (from 0.53 to 0.88 ), these results indicate that additional cross-section measurements or evaluations may be required to improve the high-energy cross sections. In the meantime, however, the newer set is recommended over the old for cases where the source incident on the shield is rather hard and the shield contains thick $\mathrm{B}_{4} \mathrm{C}$ regions. Calculations of the Alternate Shielding Materials Experiment ${ }^{11,12}$ configurations containing $B_{4} C$ with the old and new cross-section sets showed little difference in the results, mainly because fast neutrons were only a small part of the source incident on the $\mathrm{B}_{4} \mathrm{C}$. For Configuration II.D, the TSR-I neutron spectrum was hardened by the $\mathrm{UO}_{2}$ blankets and thermalized little by the steel preceding the first $\mathrm{B}_{4} \mathrm{C}$ slab. The normalized neutron spectrum behind the blankets in the near-core spectrum modifier (Configuration I.A) is compared to that behind deep-pool sodium spectrum modifier (Configuration V.A) in Fig. 8. The configuration I.A spectrum is rather hard with most of the flux above $3 \mathrm{keV}$ while the configuration V.A spectrum is much softer.

\subsubsection{Bonner Ball Count Rates at Spectrometer Locations}

Calculated and Measured 3-, 4-, 5-, and 10-in Bonner ball count rates behind configurations at the same locations where spectrum measurements were taken are shown in Table 3. For Configuration I.A, the agreement is excellent for the 3-and 5-in Bonner balls and good for the 10-in Bonner ball. For the other configurations the agreement for the 4-, 5-, and 10-in Bonner balls is generally good, except for the fair agreement for the 
ORNL-DWG 88-6236

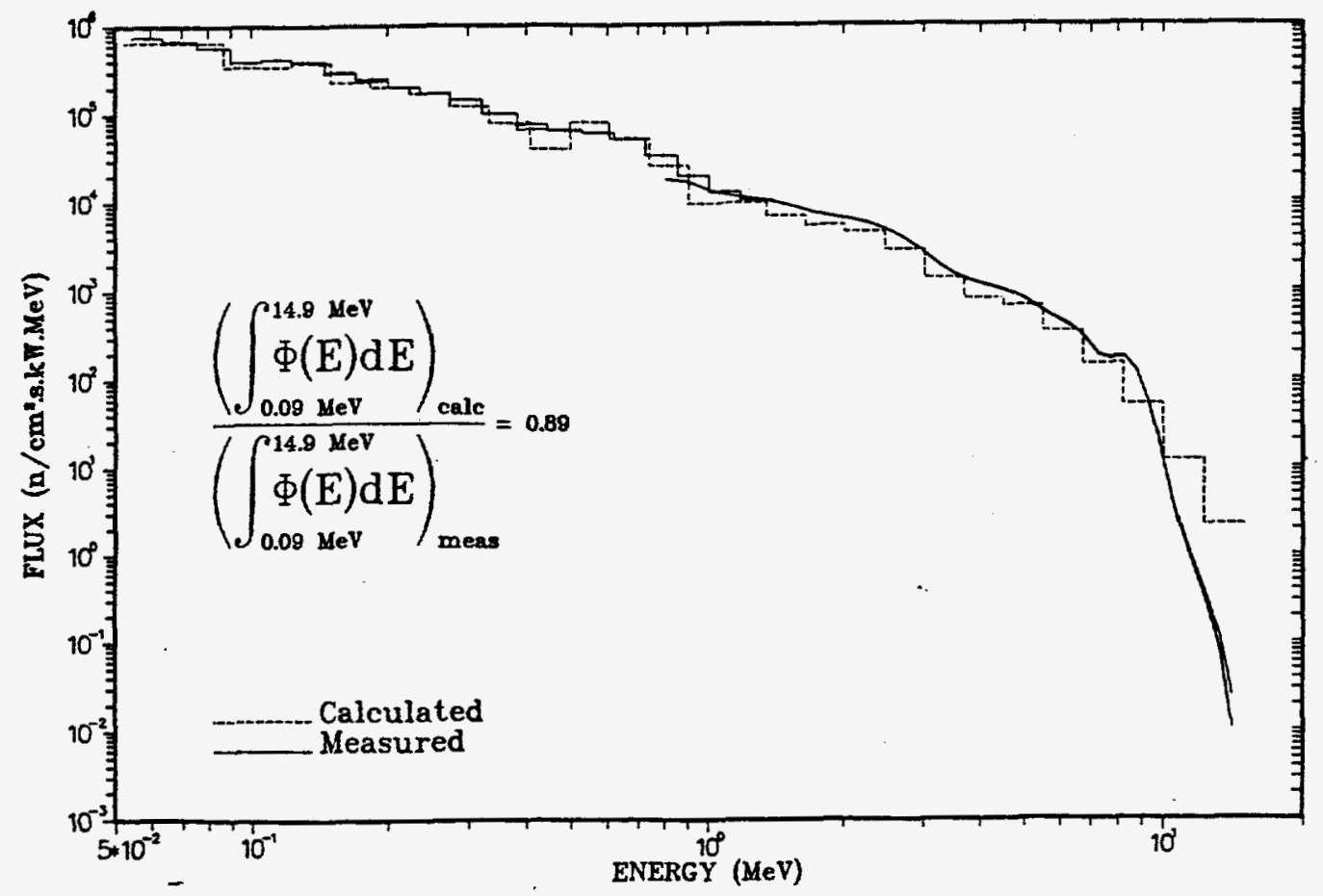

Figure 3. Calculated versus measured $\mathrm{E}>0.05 \mathrm{MeV}$ neutron spectra on centerline $178.8 \mathrm{~cm}$ behind Configuration I.A. 


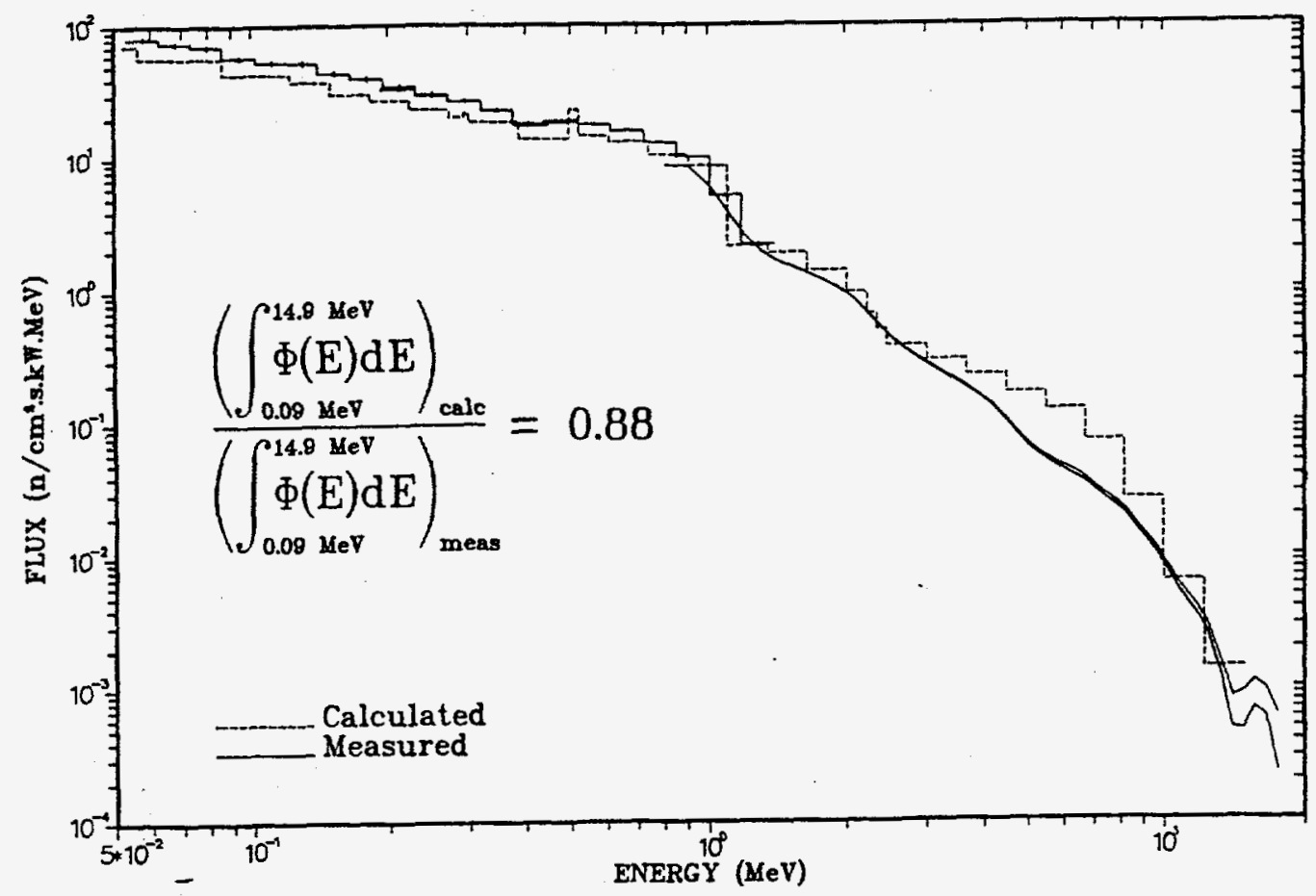

Fig. 4. Calculated versus measured $\mathrm{E}>0.05 \mathrm{MeV}$ neutron spectra on centerline $47.3 \mathrm{~cm}$ behind Configuration II.D.

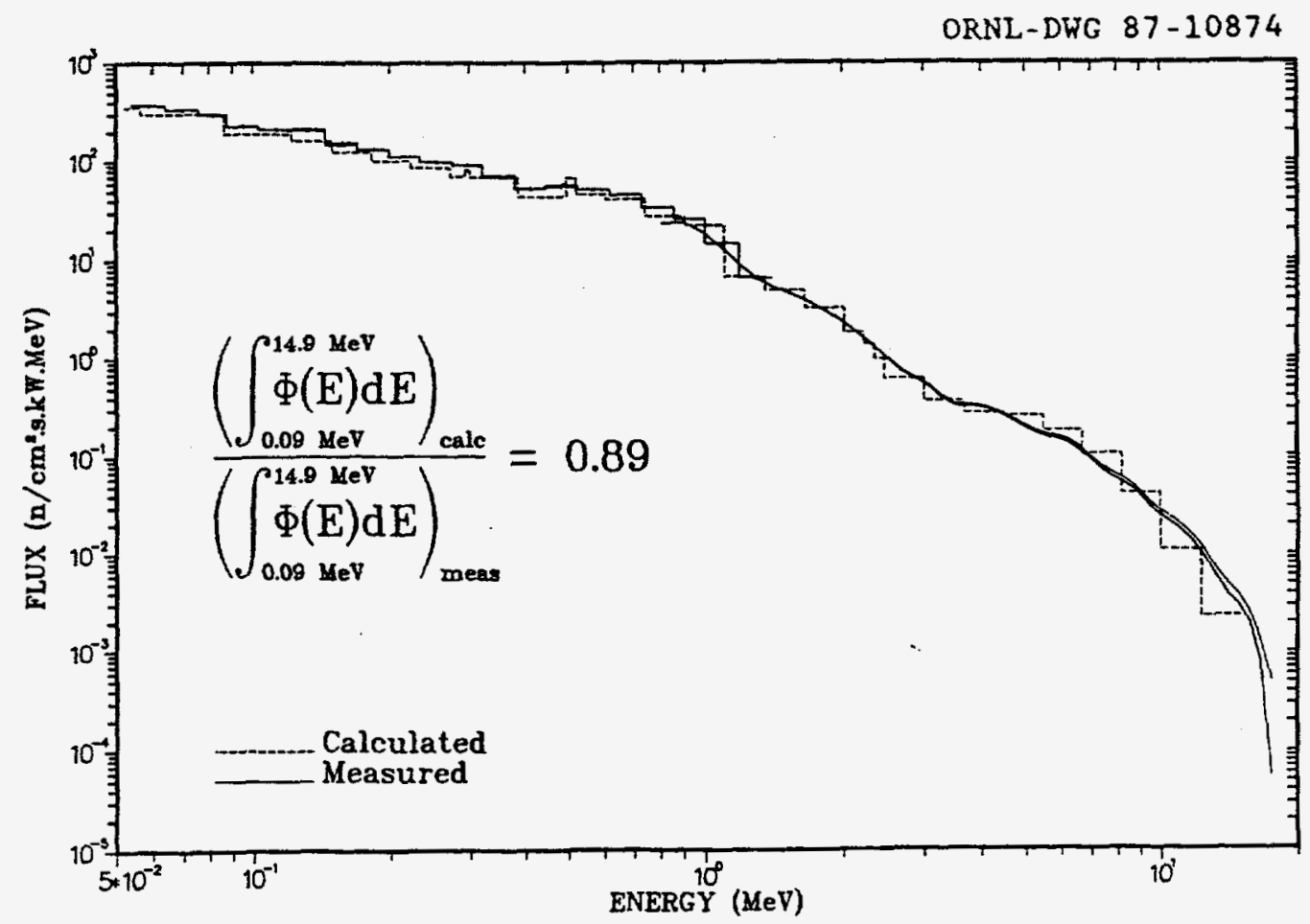

Fig. 5. Calculated versus measured $\mathbf{E}>0.05 \mathrm{MeV}$ neutron spectra on centerline $42.8 \mathrm{~cm}$ behind Configuration IV.C. 
ORNL-DWG 87-10871

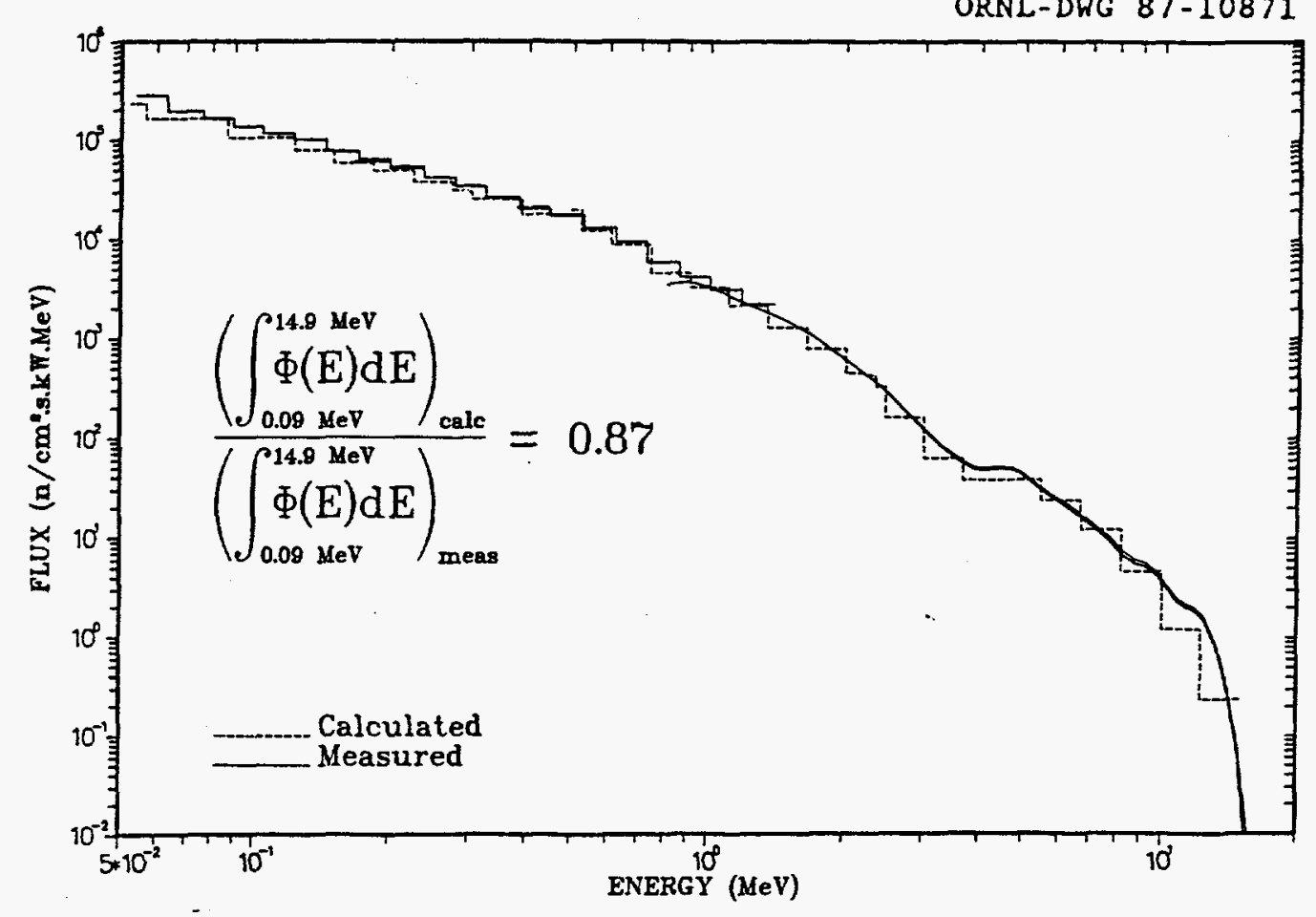

Fig. 6. Calculated versus measured $\mathrm{E}>0.05 \mathrm{MeV}$ neutron spectra on centerline $51.1 \mathrm{~cm}$ behind Configuration VI.A.

ORNL-DWG 87-10873

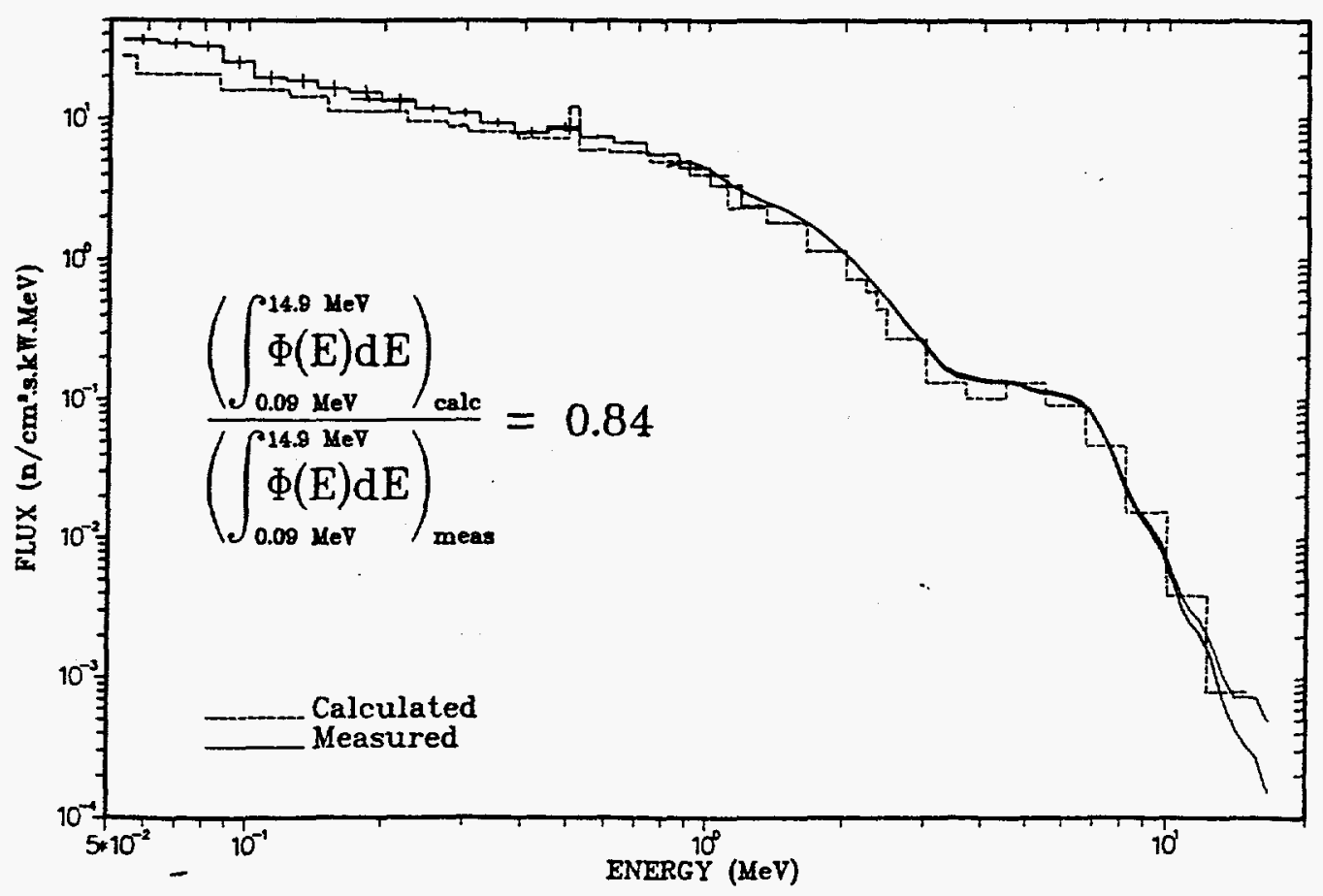

Fig. 7. Calculated versus measured $E>0.05 \mathrm{MeV}$ neutron spectra on centerline $63.2 \mathrm{~cm}$ behind Configuration VI.F. 


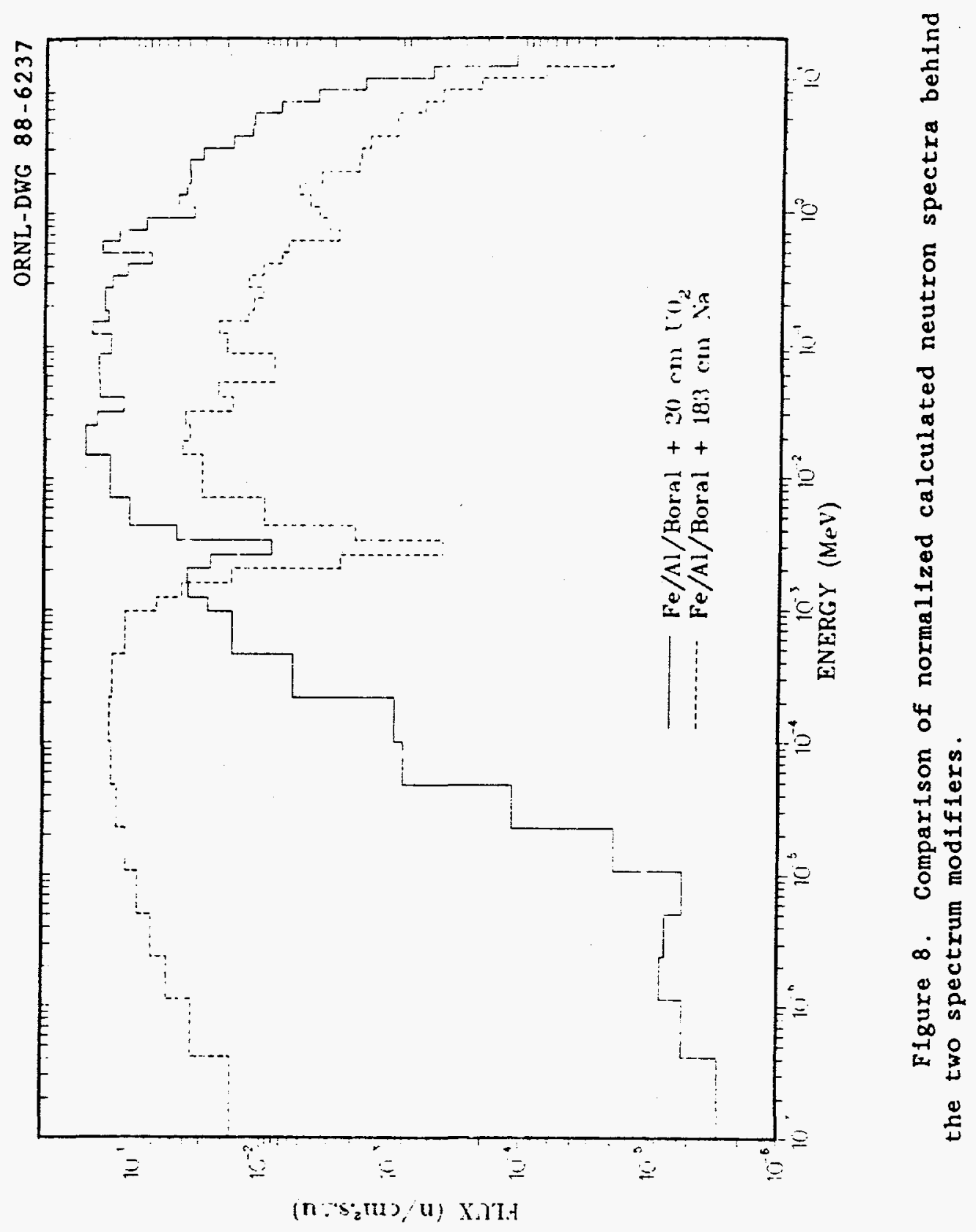


Table 3. Comparison of Calculated and Measured Bonner Ball Count Rates $\left(\mathrm{s}^{-1} \cdot \mathrm{W}_{+}^{-1}\right)$ at the Spectrum Measurement Locations

\begin{tabular}{|c|c|c|c|c|}
\hline \multirow[b]{2}{*}{ Configuration } & \multicolumn{4}{|c|}{ Detector } \\
\hline & 3-in. BB & 4-in. BB & 5-in. BB & 10-in. BB \\
\hline \multicolumn{5}{|l|}{ I.A } \\
\hline Calculated & $6.44(+1)^{a}$ & & $3.03(+2)$ & $1.02(+2)$ \\
\hline Measured & $6.86(+1)$ & & $3.13(+2)$ & $1.20(+2)$ \\
\hline$C / E^{b}$ & 0.94 & & 0.97 & 0.85 \\
\hline \multicolumn{5}{|l|}{ II.D } \\
\hline Calculated & $4.58(-3)$ & & $3.26(-2)$ & $1.77(-2)$ \\
\hline $\begin{array}{l}\text { Measured } \\
\text { C/E }\end{array}$ & $\begin{array}{l}6.68(-3) \\
0.69\end{array}$ & & $\begin{array}{l}4.10(-2) \\
0.80\end{array}$ & $\begin{array}{l}2.07(-2) \\
0.86\end{array}$ \\
\hline \multicolumn{5}{|l|}{ IV.C } \\
\hline Calculated & $2.61(-2)$ & & $1.44(-1)$ & $5.90(-2)$ \\
\hline Measured & $3.36(-2)$ & & $1.76(-1)$ & $6.95(-2)$ \\
\hline $\mathrm{C} / \mathrm{E}$ & 0.78 & & 0.82 & \\
\hline \multicolumn{5}{|l|}{ VI.A } \\
\hline Calculated & & $2.04(+2)$ & $2.25(+2)$ & $4.42(+1)$ \\
\hline Measured & & $2.56(+2)$ & $2.82(+2)$ & $6.00(+1)$ \\
\hline$C / E$ & & 0.80 & 0.80 & 0.74 \\
\hline \multicolumn{5}{|l|}{ VI.F } \\
\hline Calculated & $3.34(-3)$ & & $1.80(-2)$ & $9.54(-3)$ \\
\hline Measured & $4.51(-3)$ & & $2.22(-2)$ & $1.12(-2)$ \\
\hline $\mathrm{C} / \mathrm{E}$ & 0.74 & & 0.81 & 0.85 \\
\hline
\end{tabular}

${ }^{a}$ Read as $6.44 \times 10^{+1}$.

${ }^{b}$ Calculation-to-experiment ratio. 
10-in Bonner ball behind Configuration VI.A. The agreement is fair for the 3-in Bonner ball behind Configurations II.D, IV.C, and VI.F. The reasons for the low C/E's is not understood, particularly since, as will be seen later, C/E's were much better at $30 \mathrm{~cm}$ behind those same configurations in the absence of the lead required to make the spectrum measurements. While this suggests the lead cross sections may be causing the problems, unaccounted background may also be contributing to the discrepancy. Configuration I.A results had background subtracted and the agreement was excellent. Background was not measured at the close-in detector positions because the configuration mockups are thought to shield the detector from radiation coming around the concrete peripheral shield. Yet measurements for some of the Configurations IV did show significant background even for detector positions close behind the configurations.

\subsubsection{Bonner Ball Count Rates $30 \mathrm{~cm}$ Behind Configurations}

Calculated and measured count rates are compared in Tables 4-9 for the bare and Cd-covered $\mathrm{BF}_{3}$ detectors and the 3-, 5-, 8-, and 10-in Bonner balls, respectively. Except for the special case for Configuration II.A, the calculated results for Configurations I, II, III, and V were obtained using the 51-group cross-section library. Thus, the results for the larger Configurations II are naturally low because of the " $\mathrm{B}$ problem mentioned earlier. The results for the other configurations were obtained using the 61-group crosssection library.

The worst agreement between calculated and measured results is generally seen for the bare and $\mathrm{Cd}$-covered $\mathrm{BF}_{3}$ detectors (Tables 4 and 5). For Configuration II.E, the agreement between the calculated and measured bare and $\mathrm{Cd}$-covered $\mathrm{BF}_{3}$ detector count rates is very poor with $C / E$ values of 0.06 and 0.28 , respectively. The disagreement is attributed in part to the use of the old ${ }^{11} \mathrm{~B}$ cross sections as explained in section 3.2.2. Also, due to the presence of large amounts of $\mathrm{B}_{4} \mathrm{C}$ in the configuration, the low-energy neutron flux transmitted through the shield is less than or comparable to that transmitted through the surrounding concrete or that air-scattered around the configuration. Therefore, background radiation can make the measured result considerably higher than the calculated result. The Configurations $\mathrm{V}$ containing $\mathrm{B}_{4} \mathrm{C}$ also show low $\mathrm{C} / \mathrm{E}$ values for these detectors for the same reasons given above. For Configuration VII.D, the low C/E values are attributed to unaccounted background.

For the other detectors, the agreement is good to excellent except for 1) the underpredictions behind some Configurations $I I$ and $I V, 2)$ the overpredictions behind Configurations V.E-V.J, and 3) the fair-to-good agreement behind Configurations VII. Again, the underpredictions for Configurations $\Pi$ are attributed to the use of the old ${ }^{11} B$ cross sections and background problems. Some Configurations IV also have background problems as Tables 6-9 show $C / E$ improvement for cases where background was removed from measurements close behind the configurations. A reason for the overprediction behind Configurations V.E.V.J is not apparent. Perhaps, some combination of peaks and valleys in the sodium and $B_{4} C$ cross sections allow preferential transmission of neutrons in certain energy ranges and the multigroup cross sections do not model the cross sections well enough to accurately calculate the neutron transmission. The use of the broad-group structure to calculate neutron transport through the thick steel region probably causes the part of the problem for Configurations VII. 
Table 4. Comparison of Calculated and Measured Bare $\mathrm{BF}_{3}$ Detector Count Rates $\left(\mathrm{s}^{-1} \cdot \mathrm{W}^{-1}\right)$ Close Behind Configurations

\begin{tabular}{lclll}
\hline & $\begin{array}{c}\text { Distance }(\mathrm{cm}) \\
\text { behind } \\
\text { Configuration }\end{array}$ & Calculated & Measured & C/E \\
\hline I.A & 30 & $7.73(-5)^{b}$ & $1.30(-3)$ & 0.06 \\
V.A & 30 & $4.79(+0)$ & $5.98(+0)$ & 0.80 \\
V.B & 30 & $1.25(+0)$ & $1.61(+0)$ & 0.78 \\
V.C & 30 & $7.46(-1)$ & $9.37(-1)$ & 0.80 \\
V.D & 30 & $3.41(-1)$ & $4.12(-1)$ & 0.83 \\
V.E & 30 & $2.27(-3)$ & $1.09(-2)$ & 0.21 \\
V.F & 30 & $6.14(-3)$ & $1.33(-2)$ & 0.46 \\
V.G & 30 & $3.64(-3)$ & $8.53(-3)$ & 0.43 \\
V.H & 30 & $5.53(-4)$ & $3.77(-3)$ & 0.15 \\
V.I & 30 & $1.62(-3)$ & $3.79(-3)$ & 0.43 \\
V.J & 30 & $1.41(-3)$ & $3.11(-3)$ & 0.45 \\
VI.A(3-5) & 30 & $2.41(+1)$ & $2.87(+1)$ & 0.84 \\
VI.B & 30 & $7.65(+1)$ & $8.40(+1)$ & 0.91 \\
VI.C & 30 & $9.70(+1)$ & $1.19(+2)$ & 0.82 \\
VI.D & 30 & $8.76(+1)$ & $1.13(+2)$ & 0.78 \\
VI.E & 30 & $6.63(+1)$ & $9.70(+1)$ & 0.68 \\
VI.F(3-5) & 30 & $4.50(+1)$ & $7.52(+1)$ & 0.60 \\
VII.D & 30 & $3.78(-3)$ & $1.01(-2)$ & 0.37 \\
\hline
\end{tabular}

${ }^{a}$ Calculation-to-experiment ratio.

${ }^{b}$ Read as $7.73 \times 10^{-5}$. 
Table 5. Comparison of Calculated and Measured Cd-Covered $\mathrm{BF}_{3}$ Detector Count Rates $\left(\mathrm{s}^{-1} \cdot \mathrm{W}^{-1}\right)$ Close Behind Configurations

\begin{tabular}{lclll}
\hline Configuration & $\begin{array}{c}\text { Distance }(\mathrm{cm}) \\
\text { behind } \\
\text { configuration }\end{array}$ & Calculated & Measured & C/E \\
\hline I.A & 30 & $4.28(-5)^{b}$ & $1.53(-4)$ & 0.28 \\
V.A & 30 & $2.73(+0)$ & $2.92(+0)$ & 0.93 \\
V.B & 30 & $9.97(-1)$ & $1.11(+0)$ & 0.90 \\
V.C & 30 & $4.72(-1)$ & $5.23(-1)$ & 0.90 \\
V.D & 30 & $2.01(-1)$ & $2.21(-1)$ & 0.91 \\
V.E & 30 & $2.22(-3)$ & $4.32(-3)$ & 0.51 \\
V.F & 30 & $3.20(-3)$ & $4.91(-3)$ & 0.65 \\
V.G & 30 & $2.38(-3)$ & $3.49(-3)$ & 0.68 \\
V.H & 30 & $5.12(-4)$ & $1.02(-3)$ & 0.50 \\
V.I & 30 & $9.35(-4)$ & $1.18(-3)$ & 0.79 \\
V.J & 30 & $8.73(-4)$ & $9.23(-4)$ & 0.95 \\
VI.A(3-5) & 30 & $9.28(+0)$ & $1.25(+1)$ & 0.74 \\
VI.B & 30 & $9.93(+0)$ & $1.30(+1)$ & 0.76 \\
VI.C & 30 & $5.68(+0)$ & $7.42(+0)$ & 0.77 \\
VI.D & 30 & $2.44(+0)$ & $3.09(+0)$ & 0.79 \\
VI.E & 30 & $8.61(-1)$ & $1.08(+0)$ & 0.80 \\
VI.F(3-5) & 30 & $2.65(-1)$ & $3.39(-1)$ & 0.78 \\
VII.D & 30 & $2.19(-3)$ & $4.00(-3)$ & 0.55 \\
\hline
\end{tabular}

${ }^{a}$ Calculation-to-experiment ratio.

${ }^{b}$ Read as $4.28 \times 10^{-5}$. 
Table 6. Comparison of Calculated and Measured 3-in Bonner Ball Count Rates $\left(\mathrm{s}^{-1} . \mathrm{W}^{-1}\right)$ Close Behind Configurations

\begin{tabular}{|c|c|c|c|c|}
\hline Configuration & $\begin{array}{l}\text { Distance }(\mathrm{cm}) \text { Behind } \\
\text { Configuration }\end{array}$ & Calculated & Measured & $C / E^{\bullet}$ \\
\hline I.A & 30 & $6.06(+2)^{6}$ & $5.92(+2)$ & 1.02 \\
\hline II. $A^{c}$ & 30 & $1.91(+2)$ & $2.00(+2)$ & 0.96 \\
\hline II. $A^{d}$ & 30 & $1.79(+2)$ & $200(+2)$ & 0.90 \\
\hline II.B & 30 & $3.86 x+0)$ & $4.35(+0)$ & 0.89 \\
\hline II.C & 30 & $1.41(-1)$ & $1.78(-1)$ & 0.79 \\
\hline II.D(3-5) & 30 & $6.19(-3)$ & $1.03(-2)$ & 0.60 \\
\hline II.E & 30 & $3.10(-3)$ & $5.20(-3)$ & 0.60 \\
\hline III.A & 30 & $3.22(+2)$ & $288(+2)$ & 1.12 \\
\hline III.B & 30 & $7.07(+1)$ & $7.26(+1)$ & 0.97 \\
\hline III.C & 30 & $1.44(+2)$ & $1.55(+2)$ & 0.93 \\
\hline III.D & 30 & $3.10(+1)$ & $3.49(+1)$ & 0.89 \\
\hline III.E & 30 & $9.10(+0)$ & $1.07(+1)$ & 0.85 \\
\hline IV.A & 30 & $9.65(+1)$ & $1.06(+2)$ & 0.91 \\
\hline IV.B & 30 & $2.15(+1)$ & $240(+1)$ & 0.90 \\
\hline IV.C(3-5) & 30 & $4.54(-2)$ & $5.09(-2)$ & 0.89 \\
\hline IV.D & 30 & $2.83(-2)$ & $3.11(-2)$ & 0.91 \\
\hline IV.E & 30 & $1.80(-2)$ & $1.91(-2)$ & 0.94 \\
\hline IV.F & 30 & $4.51(-3)$ & $5.43(-3)$ & 0.83 \\
\hline IV.F & 72.4 & $2.08(-3)$ & $2.41(-3)$ & 0.86 \\
\hline IV.G & 30 & $7.68(-4)$ & $1.26(-3)$ & 0.61 \\
\hline IV.G $G^{e}$ & 53.6 & $4.65(-4)$ & $6.67(-4)$ & 0.70 \\
\hline IV.H & 30 & $284(-4)$ & $7.15(-4)$ & 0.40 \\
\hline IV.He & 52.1 & $1.78(-4)$ & $2.95(-4)$ & 0.60 \\
\hline IVI & 30 & $1.20(-4)$ & $5.07(-4)$ & 0.24 \\
\hline IV.I & 49.5 & $8.17(-5)$ & $1.52(-4)$ & 0.54 \\
\hline V.A & 30 & $3.84(+1)$ & $3.44(+1)$ & 1.12 \\
\hline V.B & 30 & $1.33(+1)$ & $1.25(+1)$ & 1.06 \\
\hline V.C & 30 & $5.52(+0)$ & $5.42(+0)$ & 1.02 \\
\hline V.D & 30 & $213(+0)$ & $1.97(+0)$ & 1.08 \\
\hline V.E & 30 & $2.25(-1)$ & $1.99(-1)$ & 1.13 \\
\hline V.F & 30 & $1.32(-1)$ & $1.27(-1)$ & 1.04 \\
\hline V.G & 30 & $6.85(-2)$ & $6.33(-2)$ & 1.08 \\
\hline V.H & 30 & $6.38(-2)$ & $5.43(-2)$ & 1.17 \\
\hline V.I & 30 & $4.26(-2)$ & $3.40(-2)$ & 1.25 \\
\hline VJ & 30 & $2.46(-2)$ & $1.34(-2)$ & 1.84 \\
\hline VI.A(3-5) & .30 & $1.89(+2)$ & $2.01(+2)$ & 0.94 \\
\hline VI.B & 30 & $1.25(+2)$ & $1.35(+2)$ & 0.93 \\
\hline V.c & 30 & $5.70(+1)$ & $6.30(+1)$ & 0.90 \\
\hline VI.D & 30 & $2.11(+1)$ & $2.23(+1)$ & 0.95 \\
\hline VI.E & 30 & $6.74(+0)$ & $7.44(+0)$ & 0.91 \\
\hline VI. $F(3-5)$ & 30 & $1.93(+0)$ & $2.10(+0)$ & 0.92 \\
\hline VII.A & 30 & $5.82(+1)$ & $6.57(+1)$ & 0.89 \\
\hline VII.B & 30 & $1.57(+1)$ & $1.92(+1)$ & 0.82 \\
\hline VIl.C & 30 & $2.31(-1)$ & $3.04(-1)$ & 0.76 \\
\hline VII.D & 30 & $1.25(-1)$ & $1.52(-1)$ & 0.82 \\
\hline
\end{tabular}

${ }^{2}$ Calculation-to-experiment ratio.

${ }^{b}$ Read as $6.06 \times 10^{+2}$.

'Calculated result obtained using 51 groups.

'Calculated result obtained using 61 groups.

The measured result has background subtracted. 
Table 7. Comparison of Calculated and Measured 5-in. Bonner Ball Count Rates $\left(s^{-1} \cdot W^{-1}\right)$ Close Behind Configurations

\begin{tabular}{|c|c|c|c|c|}
\hline Configuration & $\begin{array}{c}\text { Distance }(\mathrm{cm}) \text { behind } \\
\text { configuration }\end{array}$ & Calculated & Measured & $C / E^{\prime}$ \\
\hline I.A & 30 & $294(+3)^{6}$ & $286(+3)$ & 1.08 \\
\hline II. $A^{c}$ & 30 & $7.64(+2)$ & $8.31(+2)$ & 0.92 \\
\hline II. $A^{d}$ & 30 & $7.50(+2)$ & $8.31(+2)$ & 0.90 \\
\hline II.B & 30 & $218(+1)$ & $264(+1)$ & 0.83 \\
\hline II.C & 30 & $8.65(-1)$ & $1.26(+0)$ & 0.69 \\
\hline II.D(3-5) & 30 & $4.20(-2)$ & $7.16(-2)$ & 0.59 \\
\hline II.E & 30 & $1.47(-2)$ & $247(-2)$ & 0.60 \\
\hline III.A & 30 & $1.32(+3)$ & $1.33(+3)$ & 0.99 \\
\hline III.B & 30 & $3.76(+2)$ & $4.12(+2)$ & 0.91 \\
\hline III.C & 30 & $5.59(+2)$ & $6.26(+2)$ & 0.89 \\
\hline III.D & 30 & $1.68(+2)$ & $2.02(+2)$ & 0.83 \\
\hline III.E & 30 & $5.07(+1)$ & $6.13(+1)$ & 0.83 \\
\hline IV.A & 30 & $218(+2)$ & $2.45(+2)$ & 0.89 \\
\hline IV.B & 30 & $3.81(+1)$ & $4.42(+1)$ & 0.86 \\
\hline IV.C(3-5) & 30 & $2.61(-1)$ & $298(-1)$ & 0.88 \\
\hline TV.D & 30 & $1.44(-1)$ & $1.64(-1)$ & 0.88 \\
\hline IV.E & 30 & $4.45(-2)$ & $4.93(-2)$ & 0.90 \\
\hline IV.F & 30 & $8.55(-3)$ & $1.08(-2)$ & 0.79 \\
\hline IV.F & 72.4 & $3.97(-3)$ & $4.72(-3)$ & 0.84 \\
\hline IV.G & 30 & $1.30(-3)$ & $220(-3)$ & 0.59 \\
\hline IV.Ge & 53.6 & $7.98(-4)$ & $1.14(-3)$ & 0.70 \\
\hline IV.H & 30 & $5.42(-4)$ & $1.32(-3)$ & 0.41 \\
\hline IV.He & 52.1 & $3.44(-4)$ & $5.57(-4)$ & 0.62 \\
\hline IV.I & 30 & $2.08(-4)$ & $8.95(-4)$ & 0.23 \\
\hline $\mathrm{NVI}^{\mathrm{e}}$ & 49.5 & $1.42(-4)$ & $291(-4)$ & 0.49 \\
\hline V.A & 30 & $6.04(+1)$ & $5.61(+1)$ & 1.08 \\
\hline V.B & 30 & $208(+1)$ & $2.03(+1)$ & 1.02 \\
\hline V.C & 30 & $7.97(+0)$ & $7.66(+0)$ & 1.04 \\
\hline V.D & 30 & $288(+0)$ & $268(+0)$ & 1.07 \\
\hline V.E & 30 & $1.03(+0)$ & $9.02(-1)$ & 1.14 \\
\hline V.F & 30 & $4.16(-1)$ & $3.91(-1)$ & 1.06 \\
\hline V.G & 30 & $1.69(-1)$ & $1.50(-1)$ & 1.13 \\
\hline V.H & 30 & $3.37(-1)$ & $283(-1)$ & 1.19 \\
\hline V.I & 30 & $1.47(-1)$ & $1.19(-1)$ & 1.24 \\
\hline VJ & 30 & $6.31(-2)$ & $4.94(-2)$ & 1.28 \\
\hline VI.A(3-5) & 30 & $4.58(+2)$ & $5.15(+2)$ & 0.89 \\
\hline VI.B & 30 & $2.16(+2)$ & $2.46(+2)$ & 0.88 \\
\hline VI.C & 30 & $8.23(+1)$ & $9.45(+1)$ & 0.87 \\
\hline VI.D & 30 & $271(+1)$ & $2.97(+1)$ & 0.91 \\
\hline VIE & 30 & $8.00(+0)$ & $9.04(+0)$ & 0.88 \\
\hline V.F(3-5) & 30 & $219(+0)$ & $242(+0)$ & 0.90 \\
\hline VIIA & 30 & $2.13(+2)$ & $2.61(+2)$ & 0.82 \\
\hline VII.B & 30 & $5.59(+1)$ & $7.69(+1)$ & 0.73 \\
\hline VII.C & 30 & $1.20(+0)$ & $1.61(+0)$ & 0.75 \\
\hline VII.D & 30 & $4.36(-1)$ & $5.46(-1)$ & 0.80 \\
\hline
\end{tabular}

Calculation-to-experiment ratio.

read as $294 \times 10^{+3}$.

'Calculated result obtained using 51 groups.

¿Calculated result obtained using 61 groups.

The measured result has background subtracted. 
Table 8. Comparison of Calculated and Measured 8-in. Bonner

Ball Count Rates $\left(8^{-1} \cdot W^{-1}\right)$ Clase Behind Configurations

\begin{tabular}{|c|c|c|c|c|}
\hline Configuration & $\begin{array}{c}\text { Distance }(\mathrm{cm}) \text { behind } \\
\text { configuration }\end{array}$ & Calculated & Measured & $C / E^{\prime}$ \\
\hline IA & 30 & $2.01(+3)^{6}$ & $2.10(+3)$ & 0.96 \\
\hline II. $A^{c}$ & 30 & $4.95(+2)$ & $5.68(+2)$ & 0.87 \\
\hline II. $A^{d}$ & 30 & $5.02(+2)$ & $5.68(+2)$ & 0.88 \\
\hline II.B & 30 & $1.62(+1)$ & $2.04(+1)$ & 0.79 \\
\hline II.C & 30 & $6.94(-1)$ & $1.02(+0)$ & 0.68 \\
\hline II.D(3-5) & 30 & $3.73(-2)$ & $6.68(-2)$ & 0.56 \\
\hline II.E & 30 & $1.08(-2)$ & $1.85(-2)$ & 0.58 \\
\hline III. $\mathbf{A}$ & 30 & $8.65(+2)$ & $9.17(+2)$ & 0.94 \\
\hline III.B & 30 & $2.71(+2)$ & $3.17(+2)$ & 0.85 \\
\hline III.C & 30 & $3.60(+2)$ & $4.24(+2)$ & 0.85 \\
\hline III.D & 30 & $1.18(+2)$ & $1.47(+2)$ & 0.80 \\
\hline III.E & 30 & $3.61(+1)$ & $4.57(+1)$ & 0.79 \\
\hline IV.A & 30 & $1.06(+2)$ & $1.25(+2)$ & 0.85 \\
\hline IV.B & 30 & $1.60(+1)$ & $1.87(+1)$ & 0.86 \\
\hline N.C(3-5) & 30 & $203(-1)$ & $2.15(-1)$ & 0.94 \\
\hline IV.D & 30 & $1.06(-1)$ & $1.21(-1)$ & 0.88 \\
\hline TV.E & 30 & $2.34(-2)$ & $2.64(-2)$ & 0.89 \\
\hline IV.F & 30 & $3.86(-3)$ & $4.82(-3)$ & 0.80 \\
\hline IV.FE & 72.4 & $1.82(-3)$ & $2.20(-3)$ & 0.83 \\
\hline IV.G & 30 & $5.52(-4)$ & $9.34(-4)$ & 0.59 \\
\hline IV.G $G^{e}$ & 53.6 & $3.44(-4)$ & $4.90(-4)$ & 0.70 \\
\hline IV.H & 30 & $252(-4)$ & $5.83(-4)$ & 0.43 \\
\hline IV.He & 52.1 & $1.63(-4)$ & $2.48(-4)$ & 0.66 \\
\hline IV.I & 30 & $8.67(-5)$ & $3.73(-4)$ & 0.23 \\
\hline IV.I & 49.5 & $6.03(-5)$ & $1.20(-4)$ & 0.50 \\
\hline V.A & 30 & $2.20(+1)$ & $2.12(+1)$ & 1.04 \\
\hline V.B & 30 & $7.86(+0)$ & $7.94(+0)$ & 0.99 \\
\hline V.C & 30 & $2.85(+0)$ & $2.79(+0)$ & 1.02 \\
\hline V.D & 30 & $9.88(-1)$ & $9.37(-1)$ & 1.05 \\
\hline V.E & 30 & $7.06(-1)$ & $5.93(-1)$ & 1.19 \\
\hline V.F & 30 & $2.35(-1)$ & $2.12(-1)$ & 1.11 \\
\hline V.G & 30 & $8.16(-2)$ & $7.20(-2)$ & 1.13 \\
\hline V.H & 30 & $258(-1)$ & $2.11(-1)$ & 1.22 \\
\hline V.I & 30 & $9.01(-2)$ & $7.18(-2)$ & 1.25 \\
\hline VJ & 30 & $3.22(-2)$ & $2.52(-2)$ & 1.28 \\
\hline VI.A(3-5) & 30 & $2.25(+2)$ & $261(+2)$ & 0.86 \\
\hline VI.B & 30 & $8.76(+1)$ & $1.03(+2)$ & 0.85 \\
\hline VI.C & 30 & $3.03(+1)$ & $3.62(+1)$ & 0.84 \\
\hline VI.D & 30 & $9.46(+0)$ & $1.06(+1)$ & 0.89 \\
\hline VI.E & 30 & $272(+0)$ & $3.08(+0)$ & 0.88 \\
\hline VI.F(3-5) & 30 & $7.44(-1)$ & $8.44(-1)$ & 0.88 \\
\hline VIIA & 30 & $1.34(+2)$ & $1.69(+2)$ & 0.79 \\
\hline VII.B & 30 & $3.38(+1)$ & $4.64(+1)$ & 0.73 \\
\hline VII.C & 30 & $7.90(-1)$ & $1.08(+0)$ & 0.73 \\
\hline VII.D & 30 & $2.37(-1)$ & $3.11(-1)$ & 0.76 \\
\hline
\end{tabular}

'Calculation-to-experiment ratio.

${ }^{b}$ Read as $2.01 \times 10^{+3}$.

'Calculated result obtained using 51 groups.

${ }^{\circ}$ Calculated result obtained using 61 groups.

'The measured result has background subtracted. 
Table 9. Comparison of Calculated and Measured 10-in. Bonner Ball Count Rates $\left(\mathrm{s}^{-1} \cdot \mathrm{W}^{-1}\right)$ Close Behind Configurations

\begin{tabular}{|c|c|c|c|c|}
\hline Configuration & $\begin{array}{c}\text { Distance }(\mathrm{cm}) \text { behind } \\
\text { configuration }\end{array}$ & Calculated & Measured & $C / E^{*}$ \\
\hline I.A & 30 & $1.00(+3)^{6}$ & $1.13(+3)$ & 0.89 \\
\hline II. A $^{\mathrm{C}}$ & 30 & $2.37(+2)$ & $271(+2)$ & 0.87 \\
\hline II. $A^{d}$ & 30 & $2.44(+2)$ & $271(+2)$ & 0.90 \\
\hline II.B & 30 & $8.23(+0)$ & $1.07(+1)$ & 0.77 \\
\hline II.C & 30 & $3.75(-1)$ & $5.76(-1)$ & 0.65 \\
\hline II.D(3-5) & 30 & $2.17(-2)$ & $3.89(-2)$ & 0.56 \\
\hline II.E & 30 & $5.76(-3)$ & $9.63(-3)$ & 0.60 \\
\hline IIIA & 30 & $4.18(+2)$ & $4.59(+2)$ & 0.91 \\
\hline III.B & 30 & $1.35(+2)$ & $1.63(+2)$ & 0.83 \\
\hline III.C & 30 & $1.71(+2)$ & $2.13(+2)$ & 0.80 \\
\hline III.D & 30 & $5.68(+1)$ & $7.36(+1)$ & 0.77 \\
\hline III.E & 30 & $1.75(+1)$ & $237(+1)$ & 0.74 \\
\hline IVA & 30 & $4.59(+1)$ & $5.42(+1)$ & 0.85 \\
\hline T.B & 30 & $6.64(+0)$ & $7.94(+0)$ & 0.84 \\
\hline IV.C(3-5) & 30 & $1.09(-1)$ & $1.16(-1)$ & 0.94 \\
\hline IV.D & 30 & $5.48(\cdot 2)$ & $6.35(-2)$ & 0.86 \\
\hline IV.E & 30 & $1.07(-2)$ & $1.20(-2)$ & 0.89 \\
\hline IV.F & 30 & $1.67(-3)$ & $2.10(-3)$ & 0.80 \\
\hline $\mathrm{IV}^{\mathrm{Fe}} \mathrm{e}^{\mathrm{e}}$ & 72.4 & $7.97(-4)$ & $9.41(-4)$ & 0.85 \\
\hline IV.G & 30 & $2.36(-4)$ & $3.95(-4)$ & 0.60 \\
\hline IV.G G $^{e}$ & 53.6 & $1.49(-4)$ & $203(-4)$ & 0.73 \\
\hline IV.H & 30 & $1.11(-4)$ & $248(-4)$ & 0.45 \\
\hline IV.He & 52.1 & $7.26(-5)$ & $1.04(-4)$ & 0.70 \\
\hline IV.I & 30 & $3.60(-5)$ & $1.55(-4)$ & 0.23 \\
\hline IV.I ${ }^{e}$ & 49.5 & $2.53(-5)$ & $4.70(-5)$ & 0.54 \\
\hline V.A & 30 & $8.67(+0)$ & $8.24(+0)$ & 1.05 \\
\hline V.B & 30 & $3.19(+0)$ & $3.20(+0)$ & 1.00 \\
\hline V.C & 30 & $1.13(+0)$ & $1.09(+0)$ & 1.04 \\
\hline V.D & 30 & $3.86(-1)$ & $3.65(-1)$ & 1.06 \\
\hline V.E & 30 & $3.57(-1)$ & $298(-1)$ & 1.20 \\
\hline V.F & 30 & $1.09(-1)$ & $9.76(-2)$ & 1.12 \\
\hline V.G & 30 & $3.54(-2)$ & $3.13(-2)$ & 1.13 \\
\hline V.H & 30 & $1.39(-1)$ & $1.14(-1)$ & 1.22 \\
\hline V.I & 30 & $4.38(-2)$ & $3.51(-2)$ & 1.25 \\
\hline VJ & 30 & $1.44(-2)$ & $1.16(-2)$ & 1.24 \\
\hline VI.A(3-5) & 30 & $9.66(+1)$ & $1.10(+2)$ & 0.88 \\
\hline VI.B & 30 & $3.57(+1)$ & $4.25(+1)$ & 0.84 \\
\hline VI.C & 30 & $1.21(+1)$ & $1.47(+1)$ & 0.82 \\
\hline VI.D & 30 & $3.78(+0)$ & $4.32(+0)$ & 0.88 \\
\hline VI.E & 30 & $1.10(+0)$ & $1.25(+0)$ & 0.88 \\
\hline VI.F(3-5) & 30 & $3.07(-1)$ & $3.58(-1)$ & 0.86 \\
\hline VIIA & 30 & $6.21(+1)$ & $7.77(+1)$ & 0.80 \\
\hline VII.B & 30 & $1.52(+1)$ & $213(+1)$ & 0.71 \\
\hline VII.C & 30 & $3.62(-1)$ & $5.03(-1)$ & 0.72 \\
\hline VII.D & 30 & $1.00(-1)$ & $1.37(-1)$ & 0.73 \\
\hline
\end{tabular}

${ }^{2}$ Calculation-to-experiment ratio.

${ }^{b}$ Read as $1.00 \times 10^{+3}$.

'Calculated result obtained using 51 groups.

${ }^{\circ}$ Calculated result obtained using 61 groups.

The measured result has background subtracted. 


\subsubsection{Bonner Ball Count Rates Behind Configurations at Large Distances from the TSR-II Core Center}

Calculated bare and $\mathrm{Cd}$-covered $\mathrm{BF}_{3}$ detector and 3-, 5-, 8-, and 10-in Bonner ball count rates behind configurations at large distances from the TSR-II core center are compared to measured count rates respectively in Tables $10-15$. The agreement is similar to that $30 \mathrm{~cm}$ behind the configurations but slightly worse. The agreement for those Configurations $V$ that were largely overpredicted at $30 \mathrm{~cm}$ is much better. The general downward trend in the $\mathrm{C} / \mathrm{E}$ values seems to indicate some underprediction of the fluxes at the distant detectors.

\subsubsection{Bonner Ball Count Rates Inside Configurations}

Because of high background behind the very large Configurations IV, measurements for those configurations were made with Bonner balls in a void region within the configuration mockup and completely surrounded by shielding. Configuration IV.I was measured both with and without the shielding around the detectors, while Configurations IV.J through IV.M were all measured in situ. Calculated and measured Bonner ball count rates are compared in Table 16. Poor agreement is shown for the bare $\mathrm{BF}_{3}$ detector, while fair to good agreement is shown for the $\mathrm{Cd}$-covered $\mathrm{BF}_{3}$ detector and good to excellent agreement is shown for the 3-, 5-, 8-, and 10-in Bonner balls. If Configuration IV.J is excluded, one sees improved $\mathrm{C} / \mathrm{E}$ values with increasing configuration size for all detectors except the bare detector. Boundary sources for all configurations except Configuration IV.J were derived from output from the Configuration IV.J DOT-IV calculation. This would be an explanation for higher C/E values for Configuration IV.IA and lower $\mathrm{C} / \mathrm{E}$ values for Configurations IV.K through IV.M, but not the trend shown in Table 16. A possible explanation for the upward trend in the $\mathrm{C} / \mathrm{E}$ values is an overprediction of the transport through sodium. As the thickness of sodium preceding the void increases, the $\mathrm{C} / \mathrm{E}$ also increases.

\subsubsection{5-in Bonner Ball Horizontal Traverses $30 \mathrm{~cm}$ Behind Configurations}

Plotted curves of calculated and measured count rates for 5 -in Bonner ball horizontal traverses $30 \mathrm{~cm}$ behind configurations are shown in Figs. 9-31. The calculated and measured curve shapes are in reasonably good agreement, although the calculated results tend to be more underpredictive at the outer edges of the traverse. The 2-D modeling of the square slabs probably affects the comparisons at the outer edges of the traverses. The top-of-range $\mathrm{C} / \mathrm{E}$ values are good to excellent except for Configurations II.D and II.E, for which calculations were performed with the 51-group structure and the old ${ }^{11} \mathrm{~B}$ cross section set; Configuration IV.G, for which background was high; and for Configurations VII.B and VII.C, for which agreement was fair. Note that the traverses behind Configurations V (Figs. 20-24) indicate little effect on the radial variation of the count rate due to the nonuniformity of the sodium tanks. 
Table 10. Comparison of Calculated and Measured Bare $\mathrm{BF}_{3}$ Detector Count Rates $\left(s^{-1} \cdot \mathrm{W}^{-1}\right)$ Bchind Configurations at Large Distances from the TSR-II Core Center

\begin{tabular}{|c|c|c|c|c|}
\hline Configuration & $\begin{array}{c}\text { Distance }(\mathrm{cm}) \\
\text { from TSR-II } \\
\text { core center }\end{array}$ & Calculated & Measured & $\mathrm{C} / \mathrm{E}^{\mathrm{a}}$ \\
\hline I.A & 304.8 & $8.23(-5)^{6}$ & $3.80(-4)$ & 0.22 \\
\hline V.A & 501.3 & $7.10(-1)$ & $9.40(-1)$ & 0.76 \\
\hline V.B & 501.3 & $1.75(-1)$ & $2.32(-1)$ & 0.75 \\
\hline V.C & 501.3 & $1.71(-1)$ & $1.94(-1)$ & 0.88 \\
\hline V.D & 501.3 & $1.07(-1)$ & $1.45(-1)$ & 0.74 \\
\hline V.E & 501.3 & $5.00(-4)$ & $3.70(-3)$ & 0.14 \\
\hline V.F & 501.3 & $2.12(-3)$ & $3.70(-3)$ & 0.57 \\
\hline V.G & 501.3 & $1.51(-3)$ & $3.21(-3)$ & 0.47 \\
\hline V.H & 501.3 & $1.53(-4)$ & $1.66(-3)$ & 0.09 \\
\hline V.I & 501.3 & $5.87(-4)$ & $9.40(-4)$ & 0.62 \\
\hline V.J & 501.3 & $6.94(-4)$ & $9.00(-4)$ & 0.77 \\
\hline VI.A(3-5) & 304.8 & $3.94(+0)$ & $4.98(+0)$ & 0.79 \\
\hline VI.B & 304.8 & $1.51(+1)$ & $1.69(+1)$ & 0.89 \\
\hline VI.C & 304.8 & $2.12(+1)$ & $2.79(+1)$ & 0.76 \\
\hline VI.D & 304.8 & $2.19(+1)$ & $3.07(+1)$ & 0.71 \\
\hline VI.E & 304.8 & $1.93(+1)$ & $3.10(+1)$ & 0.62 \\
\hline VI.F(3-5) & 304.8 & $1.55(+1)$ & $2.87(+1)$ & 0.54 \\
\hline VII.D & 304.8 & $3.00(-3)$ & $6.44(-3)$ & 0.47 \\
\hline
\end{tabular}

Calculation-to-experiment ratio.

${ }^{b}$ Read as $8.23 \times 10^{-5}$. 
Table 11. Comparison of Calculated and Measured $\mathrm{Cd}$-Covered $\mathrm{BF}_{3}$ Detector Count Rates $\left(\mathrm{s}^{-1} \cdot \mathrm{W}^{-1}\right)$ Behind Configurations at Large Distances from the TSR-II Core Center

\begin{tabular}{ccccc}
\hline Configuration & $\begin{array}{c}\text { Distance (cm) } \\
\text { from TSR-II } \\
\text { core center }\end{array}$ & Calculated & Measured & C/E \\
\hline I.A & 304.8 & $3.95(-5)^{6}$ & $8.30(-5)$ & 0.48 \\
V.A & 501.3 & $3.53(-1)$ & $4.34(-1)$ & 0.81 \\
V.B & 501.3 & $1.32(-1)$ & $1.58(-1)$ & 0.84 \\
V.C & 501.3 & $8.91(-2)$ & $1.13(-1)$ & 0.79 \\
V.D & 501.3 & $5.80(-2)$ & $7.14(-2)$ & 0.81 \\
V.E & 501.3 & $3.60(-4)$ & $1.20(-3)$ & 0.30 \\
V.F & 501.3 & $8.34(-4)$ & $1.40(-3)$ & 0.60 \\
V.G & 501.3 & $8.41(-4)$ & $1.34(-3)$ & 0.63 \\
V.H & 501.3 & $9.79(-5)$ & $3.85(-4)$ & 0.25 \\
V.I & 501.3 & $2.61(-4)$ & $3.56(-4)$ & 0.73 \\
V.J & 501.3 & $3.51(-4)$ & $3.65(-4)$ & 0.96 \\
VI.A(3-5) & 304.8 & $1.49(+0)$ & $2.27(+0)$ & 0.66 \\
VI.B & 304.8 & $1.90(+0)$ & $2.72(+0)$ & 0.70 \\
VI.C & 304.8 & $1.23(+0)$ & $1.79(+0)$ & 0.69 \\
VI.D & 304.8 & $6.06(-1)$ & $8.48(-1)$ & 0.71 \\
VI.E & 304.8 & $2.50(-1)$ & $3.51(-1)$ & 0.71 \\
VI.F(3-5) & 304.8 & $9.11(-2)$ & $1.27(-1)$ & 0.72 \\
VII.D & 304.8 & $1.64(-3)$ & $2.77(-3)$ & 0.59 \\
\hline
\end{tabular}

'Calculation-to-experiment ratio.

${ }^{b}$ Read as $3.95 \times 10^{-5}$. 
Table 12. Comparison of Calculated and Measured 3-in. Bonner Ball Count Rates $\left(\mathrm{s}^{-1} \cdot \mathrm{W}^{-1}\right)$ Bchind Configurations at Large Distances from the TSR-II Core Center

\begin{tabular}{|c|c|c|c|c|}
\hline Configuration & $\begin{array}{l}\text { Distance }(\mathrm{cm}) \text { behind } \\
\text { configuration }\end{array}$ & Calculated & Measured & $C / E^{*}$ \\
\hline I.A & 304.8 & $7.39(+1)^{6}$ & $7.11(+1)$ & 1.04 \\
\hline I.A & 457.2 & $2.15(+1)$ & $2.12(+1)$ & 1.01 \\
\hline II. $A^{c}$ & 304.8 & $2.94(+1)$ & $3.25(+1)$ & 0.90 \\
\hline II. $A^{d}$ & 304.8 & $2.77(+1)$ & $3.25(+1)$ & 0.85 \\
\hline Il.B & 304.8 & $7.95(-1)$ & $9.80(-1)$ & 0.81 \\
\hline II.C & 304.8 & $3.90(-2)$ & $5.35(-2)$ & 0.73 \\
\hline II.D(3-5) & 304.8 & $2.47(-3)$ & $4.26(-3)$ & 0.58 \\
\hline II.E & 304.8 & $2.43(-3)$ & $3.53(-3)$ & 0.69 \\
\hline III.A & 304.8 & $4.84(+1)$ & $4.34(+1)$ & 1.12 \\
\hline III.B & 304.8 & $1.20(+1)$ & $1.26(+1)$ & 0.95 \\
\hline III.C & 304.8 & $2.41(+1)$ & $2.64(+1)$ & 0.91 \\
\hline III.D & 304.8 & $5.88(+0)$ & $6.92(+0)$ & 0.85 \\
\hline III.E & 304.8 & $1.87(+0)$ & $2.26(+0)$ & 0.83 \\
\hline IV.A & 304.8 & $1.72(+1)$ & $2.07(+1)$ & 0.83 \\
\hline IV.B & 304.8 & $4.74(+0)$ & $6.00(+0)$ & 0.79 \\
\hline IV.C(3-5) & 304.8 & $1.49(-2)$ & $1.81(-2)$ & 0.82 \\
\hline IV.C(3-5) & 457.2 & $2.65(-3)$ & $3.72(-3)$ & 0.71 \\
\hline IV.D & 457.2 & $1.75(-3)$ & $2.36(-3)$ & 0.74 \\
\hline IV.E & 457.2 & $1.31(-3)$ & $1.68(-3)$ & 0.78 \\
\hline IV.F & 457.2 & $3.92(-4)$ & $5.06(-4)$ & 0.77 \\
\hline V.A & 501.3 & $4.77(+0)$ & $5.00(+0)$ & 0.95 \\
\hline V.B & 501.3 & $1.72(+0)$ & $1.76(+0)$ & 0.98 \\
\hline V.C & 501.3 & $1.02(+0)$ & $1.12(+0)$ & 0.91 \\
\hline V.D & 501.3 & $6.09(-1)$ & $6.22(-1)$ & 0.98 \\
\hline V.E & 501.3 & $3.11(-2)$ & $3.52(-2)$ & 0.88 \\
\hline V.F & 501.3 & $2.77(-2)$ & $3.08(-2)$ & 0.90 \\
\hline V.G & 501.3 & $2.21(-2)$ & $224(-2)$ & 0.99 \\
\hline V.H & 501.3 & $9.61(-3)$ & $1.11(-2)$ & 0.87 \\
\hline V.I & 501.3 & $9.48(-3)$ & $8.50(-3)$ & 1.12 \\
\hline V.J & 501.3 & $8.76(-3)$ & $6.86(-3)$ & 1.28 \\
\hline VI.A(3-5) & 304.8 & $3.01(+1)$ & $3.68(+1)$ & 0.82 \\
\hline VI.B & 304.8 & $2.37(+1)$ & $2.78(+1)$ & 0.85 \\
\hline VI.C & 304.8 & $1.23(+1)$ & $1.50(+1)$ & 0.82 \\
\hline VI.D & 304.8 & $5.26(+0)$ & $6.05(+0)$ & 0.87 \\
\hline VI.E & 304.8 & $1.96(+0)$ & $2.38(+0)$ & 0.82 \\
\hline VI.F(3-5) & 304.8 & $6.65(-1)$ & $7.92(-1)$ & 0.84 \\
\hline VII.A & 304.8 & $1.15(+1)$ & $1.37(+1)$ & 0.84 \\
\hline VII.B & 304.8 & $3.81(+0)$ & $5.11(+0)$ & 0.75 \\
\hline VII.C & 304.8 & $7.97(-2)$ & $1.18(-1)$ & 0.68 \\
\hline VII.D & 304.8 & $8.44(-2)$ & $1.01(-1)$ & 0.84 \\
\hline
\end{tabular}

Calculation-to-experiment ratio.

'Read as $7.39 \times 10^{+1}$.

'Calculated result obtained using 51 groups.

${ }^{d}$ Calculated result obtained using 61 groups. 
Table 13. Comparison of Calculated and Measured 5-in. Bonner Ball Count Rates $\left(s^{-1} \cdot W^{-1}\right)$ Behind Configurations at Large Distances from the TSR-II Core Center

\begin{tabular}{|c|c|c|c|c|}
\hline Configuration & $\begin{array}{l}\text { Distance }(\mathrm{cm}) \text { behind } \\
\text { configuration }\end{array}$ & Calculated & Measured & $C / E^{*}$ \\
\hline I.A & 304.8 & $3.48(+2)^{6}$ & $3.63(+2)$ & 0.96 \\
\hline I.A & 457.2 & $9.99(+1)$ & $1.13(+2)$ & 0.88 \\
\hline $11 . A^{c}$ & 304.8 & $1.15(+2)$ & $1.35(+2)$ & 0.85 \\
\hline II. $A^{d}$ & 304.8 & $1.13(+2)$ & $1.35(+2)$ & 0.84 \\
\hline II.B & 304.8 & $4.38(+0)$ & $5.94(+0)$ & 0.74 \\
\hline II.C & 304.8 & $2.41(-1)$ & $3.81(-1)$ & 0.63 \\
\hline II.D(3-5) & 304.8 & $1.68(-2)$ & $2.98(-2)$ & 0.56 \\
\hline II.E & 304.8 & $1.16(-2)$ & $1.74(-2)$ & 0.67 \\
\hline III.A & 304.8 & $1.83(+2)$ & $1.93(+2)$ & 0.95 \\
\hline III.B & 304.8 & $5.96(+1)$ & $7.15(+1)$ & 0.83 \\
\hline III.C & 304.8 & $8.88(+1)$ & $1.08(+2)$ & 0.82 \\
\hline III.D & 304.8 & $3.03(+1)$ & $3.90(+1)$ & 0.78 \\
\hline III.E & 304.8 & $1.02(+1)$ & $1.36(+1)$ & 0.75 \\
\hline IV.A & 304.8 & $3.84(+1)$ & $4.85(+1)$ & 0.79 \\
\hline IV.B & 304.8 & $8.40(+0)$ & $1.10(+1)$ & 0.76 \\
\hline IV.C(3-5) & 304.8 & $8.66(-2)$ & $1.07(-1)$ & 0.81 \\
\hline IV.C(3-5) & 457.2 & $1.51(-2)$ & $2.21(-2)$ & 0.68 \\
\hline IV.D & 457.2 & $8.79(-3)$ & $1.23(-2)$ & 0.71 \\
\hline IV.E & 457.2 & $3.20(-3)$ & $4.26(-3)$ & 0.75 \\
\hline IV.F & 457.2 & $7.39(-4)$ & $1.07(-3)$ & 0.69 \\
\hline V.A & 501.3 & $7.37(+0)$ & $8.18(+0)$ & 0.90 \\
\hline V.B & 501.3 & $2.67(+0)$ & $2.75(+0)$ & 0.97 \\
\hline V.C & 501.3 & $1.45(+0)$ & $1.61(+0)$ & 0.90 \\
\hline V.D & 501.3 & $8.18(-1)$ & $8.17(-1)$ & 1.00 \\
\hline V.E & 501.3 & $1.43(-1)$ & $1.46(-1)$ & 0.98 \\
\hline V.F & 501.3 & $8.46(-2)$ & $8.71(-2)$ & 0.97 \\
\hline V.G & 501.3 & $5.36(-2)$ & $5.15(-2)$ & 1.04 \\
\hline V.H & 501.3 & $5.08(-2)$ & $5.05(-2)$ & 1.01 \\
\hline V.I & 501.3 & $3.19(-2)$ & $2.93(-2)$ & 1.09 \\
\hline VJ & 501.3 & $220(-2)$ & $1.79(-2)$ & 1.23 \\
\hline VI.A(3-5) & 304.8 & $7.33(+1)$ & $9.29(+1)$ & 0.79 \\
\hline VI.B & 304.8 & $4.09(+1)$ & $5.10(+1)$ & 0.80 \\
\hline VI.C & 304.8 & $1.78(+1)$ & $2.24(+1)$ & 0.79 \\
\hline VI.D & 304.8 & $6.76(+0)$ & $7.94(+0)$ & 0.85 \\
\hline VI.E & 304.8 & $2.34(+0)$ & $2.89(+0)$ & 0.81 \\
\hline VI.F(3.5) & 304.8 & $7.54(-1)$ & $9.70(-1)$ & 0.78 \\
\hline VII.A & 304.8 & $4.10(+1)$ & $5.48(+1)$ & 0.75 \\
\hline VII.B & 304.8 & $1.35(+1)$ & $2.00(+1)$ & 0.68 \\
\hline VII.C & 304.8 & $4.19(-1)$ & $6.07(-1)$ & 0.69 \\
\hline VII.D & 304.8 & $2.95(-1)$ & $3.64(-1)$ & 0.81 \\
\hline
\end{tabular}

Calculation-10-experiment ratio.

${ }^{b}$ Read as $3.48 \times 10^{+2}$.

cCalculated result obtained using 51 groups.

${ }^{d}$ Calculated result obtained using 61 groups. 
Table 14. Comparison of Calculated and Measured 8-in. Bonner Ball Count Rates $\left(\mathrm{s}^{-1} \cdot \mathrm{W}^{-1}\right)$ Behind Configurations at Large Distances from the TSR-II Core Center

\begin{tabular}{|c|c|c|c|c|}
\hline Configuration & $\begin{array}{c}\text { Distance }(\mathrm{cm}) \text { behind } \\
\text { configuration }\end{array}$ & Calculated & Measured & $C / E^{*}$ \\
\hline I.A & 304.8 & $2.36(+2)^{6}$ & $2.64(+2)$ & 0.89 \\
\hline I.A & 457.2 & $6.68(+1)$ & $8.05(+1)$ & 0.81 \\
\hline II. $A^{c}$ & 304.8 & $7.38(+1)$ & $9.18(+1)$ & 0.80 \\
\hline II. $A^{d}$ & 304.8 & $7.49(+1)$ & $9.18(+1)$ & 0.82 \\
\hline II.B & 304.8 & $3.30(+0)$ & $4.61(+0)$ & 0.72 \\
\hline Il.C & 304.8 & $1.98(-1)$ & $3.17(-1)$ & 0.62 \\
\hline II.D(3-5) & 304.8 & $1.53(-2)$ & $2.84(-2)$ & 0.54 \\
\hline II.E & 304.8 & $8.92(-3)$ & $1.32(-2)$ & 0.68 \\
\hline III.A & 304.8 & $1.18(+2)$ & $1.36(+2)$ & 0.87 \\
\hline III. B & 304.8 & $4.27(+1)$ & $5.46(+1)$ & 0.78 \\
\hline III.C & 304.8 & $5.67(+1)$ & $7.35(+1)$ & 0.77 \\
\hline III.D & 304.8 & $2.13(+1)$ & $2.94(+1)$ & 0.72 \\
\hline III.E & 304.8 & $7.31(+0)$ & $1.00(+1)$ & 0.73 \\
\hline IV.A & 304.8 & $1.87(+1)$ & $2.45(+1)$ & 0.76 \\
\hline IV.B & 304.8 & $3.57(+0)$ & $4.72(+0)$ & 0.76 \\
\hline IV.C (3-5) & 304.8 & $6.94(-2)$ & $7.91(-2)$ & 0.88 \\
\hline IV.C(3-5) & 457.2 & $1.19(-2)$ & $1.67(-2)$ & 0.71 \\
\hline IV.D & 457.2 & $6.52(-3)$ & $9.40(-3)$ & 0.69 \\
\hline IV.E & 457.2 & $1.70(-3)$ & $2.35(-3)$ & 0.72 \\
\hline IV.F & 457.2 & $3.37(-4)$ & $4.72(-4)$ & 0.71 \\
\hline V.A & 501.3 & $2.68(+0)$ & $3.10(+0)$ & 0.86 \\
\hline V.B & 501.3 & $1.01(+0)$ & $1.10(+0)$ & 0.92 \\
\hline V.C & 501.3 & $5.20(-1)$ & $5.86(-1)$ & 0.89 \\
\hline V.D & 501.3 & $2.82(-1)$ & $2.92(-1)$ & 0.97 \\
\hline V.E & 501.3 & $1.01(-1)$ & $9.90(-2)$ & 1.02 \\
\hline V.F & 501.3 & $4.85(-2)$ & $4.94(-2)$ & 0.98 \\
\hline V.G & 501.3 & $2.63(-2)$ & $2.55(-2)$ & 1.03 \\
\hline V.H & 501.3 & $4.00(-2)$ & $3.81(-2)$ & 1.05 \\
\hline V.I & 501.3 & $1.98(-2)$ & $1.79(-2)$ & 1.11 \\
\hline VJ & 501.3 & $1.13(-2)$ & $9.50(-3)$ & 1.19 \\
\hline VI.A(3-5) & 304.8 & $3.65(+1)$ & $4.81(+1)$ & 0.76 \\
\hline VI.B & 304.8 & $1.68(+1)$ & $2.15(+1)$ & 0.78 \\
\hline VI.C & 304.8 & $6.61(+0)$ & $8.65(+0)$ & 0.76 \\
\hline VI.D & 304.8 & $2.39(+0)$ & $2.93(+0)$ & 0.82 \\
\hline VI.E & 304.8 & $8.08(-1)$ & $1.00(+0)$ & 0.81 \\
\hline VI.F(3-5) & 304.8 & $2.61(-1)$ & $3.18(-1)$ & 0.82 \\
\hline VII.A & 304.8 & $2.55(+1)$ & $3.50(+1)$ & 0.73 \\
\hline VII.B & 304.8 & $8.23(+0)$ & $1.25(+1)$ & 0.66 \\
\hline VII.C & 304.8 & $2.83(-1)$ & $4.16(-1)$ & 0.68 \\
\hline VII.D & 304.8 & $1.65(-1)$ & $2.05(-1)$ & 0.80 \\
\hline
\end{tabular}

${ }^{2}$ Calculation-to-experiment ratio.

${ }^{\text {bRead as }} 2.36 \times 10^{42}$.

'Calculated result obtained using 51 groups.

${ }^{\circ}$ Calculated result obtained using 61 groups. 
Table 15. Comparison of Calculated and Measured 10-in. Bonner Ball Count Rates $\left(s^{-1} \cdot W^{-1}\right)$ Behind Configurations at Large Distances from the TSR-II Core Center

\begin{tabular}{|c|c|c|c|c|}
\hline Configuration & $\begin{array}{c}\text { Distance }(\mathrm{cm}) \text { behind } \\
\text { configuration }\end{array}$ & Calculated & Measured & $\mathrm{C} / \mathrm{E}^{\prime}$ \\
\hline I.A & 304.8 & $1.17(+2)^{6}$ & $1.32(+2)$ & 0.89 \\
\hline I.A & 457.2 & $3.29(+1)$ & $4.08(+1)$ & 0.81 \\
\hline II. $A^{e}$ & 304.8 & $3.53(+1)$ & $4.39(+1)$ & 0.80 \\
\hline II. $A^{d}$ & 304.8 & $3.64(+1)$ & $4.39(+1)$ & 0.83 \\
\hline II.B & 304.8 & $1.70(+0)$ & $2.44(+0)$ & 0.70 \\
\hline II.C & 304.8 & $1.09(-1)$ & $1.81(-1)$ & 0.60 \\
\hline II.D(3-5) & 304.8 & $9.07(-3)$ & $1.65(-2)$ & 0.55 \\
\hline II.E & 304.8 & $4.96(-3)$ & $6.88(-3)$ & 0.72 \\
\hline III.A & 304.8 & $5.71(+1)$ & $6.91(+1)$ & 0.83 \\
\hline III.B & 304.8 & $2.15(+1)$ & $2.69(+1)$ & 0.80 \\
\hline III.C & 304.8 & $2.69(+1)$ & $3.63(+1)$ & 0.74 \\
\hline III.D & 304.8 & $1.04(+1)$ & $1.44(+1)$ & 0.72 \\
\hline III.E & 304.8 & $3.59(+0)$ & $5.27(+0)$ & 0.68 \\
\hline IV.A & 304.8 & $8.18(+0)$ & $1.05(+1)$ & 0.78 \\
\hline IV.B & 304.8 & $1.49(+0)$ & $1.97(+0)$ & 0.76 \\
\hline IV.C(3-5) & 304.8 & $3.79(-2)$ & $4.35(-2)$ & 0.87 \\
\hline IV.C(3-5) & 457.2 & $6.45(-3)$ & $9.12(-3)$ & 0.71 \\
\hline IV.D & 457.2 & $3.41(-3)$ & $4.94(-3)$ & 0.69 \\
\hline IV.E & 457.2 & $7.82(-4)$ & $1.05(-3)$ & 0.74 \\
\hline IV.F & 457.2 & $1.48(-4)$ & $1.94(-4)$ & 0.76 \\
\hline V.A & 501.3 & $1.05(+0)$ & $1.18(+0)$ & 0.89 \\
\hline V.B & 501.3 & $4.14(-1)$ & $4.42(-1)$ & 0.94 \\
\hline V.C & 501.3 & $2.08(-1)$ & $2.29(-1)$ & 0.91 \\
\hline V.D & 501.3 & $1.11(-1)$ & $1.17(-1)$ & 0.95 \\
\hline V.E & 501.3 & $5.23(-2)$ & $4.99(-2)$ & 1.05 \\
\hline V.F & 501.3 & $2.30(-2)$ & $2.28(-2)$ & 1.01 \\
\hline V.G & 501.3 & $1.16(-2)$ & $1.10(-2)$ & 1.05 \\
\hline V.H & 501.3 & $2.23(-2)$ & $2.08(-2)$ & 1.07 \\
\hline V.I & 501.3 & $9.90(-3)$ & $8.85(-3)$ & 1.12 \\
\hline V.J & 501.3 & $5.17(-3)$ & $4.33(-3)$ & 1.19 \\
\hline VI.A(3-5) & 304.8 & $1.59(+1)$ & $2.09(+1)$ & 0.76 \\
\hline VI.B & 304.8 & $6.89(+0)$ & $8.92(+0)$ & 0.77 \\
\hline VI.C & 304.8 & $2.67(+0)$ & $3.49(+0)$ & 0.77 \\
\hline VI.D & 304.8 & $9.67(-1)$ & $1.17(+0)$ & 0.83 \\
\hline VI.E & 304.8 & $3.31(-1)$ & $4.02(-1)$ & 0.82 \\
\hline VI.F(3-5) & 304.8 & $1.10(-1)$ & $1.34(-1)$ & 0.82 \\
\hline VII.A & 304.8 & $1.19(+1)$ & $1.61(+1)$ & 0.74 \\
\hline VII.B & 304.8 & $3.74(+0)$ & $5.63(+0)$ & 0.66 \\
\hline VII.C & 304.8 & $1.31(-1)$ & $1.98(-1)$ & 0.66 \\
\hline VII.D & 304.8 & $7.14(-2)$ & $9.03(-2)$ & 0.79 \\
\hline
\end{tabular}

${ }^{2}$ Calculation-to-experiment ratio.

${ }^{b}$ Read as $1.17 \times 10^{+2}$.

'Calculated result obtained using 51 groups.

${ }^{\mathrm{d} C a l c u l a t e d}$ result obtained using 61 groups. 
Table 16. Comparison of Calculated and Mcasured Bonner Ball Count Rates $\left(\mathrm{s}^{-1} \cdot \mathrm{W}^{-1}\right)$ in the Shielded Void Region of Several Configurations IV

\begin{tabular}{|c|c|c|c|c|c|}
\hline \multirow[b]{2}{*}{ Detector } & \multicolumn{5}{|c|}{ Configuration } \\
\hline & IV.IA & IV.J & IV.K & IV.L & IV.M \\
\hline \multicolumn{6}{|l|}{$\overline{\text { Bare } \mathrm{BF}_{3}}$} \\
\hline Calculated & $3.28(-5)^{2}$ & $1.59(-5)$ & $6.84(-6)$ & $2.90(-6)$ & $1.24(-6)$ \\
\hline Measured & $6.54(-5)$ & $2.92(-5)$ & $1.38(-5)$ & $6.18(-6)$ & $3.00(-6)$ \\
\hline $\mathrm{C} / \mathrm{E}^{\mathrm{b}}$ & 0.50 & 0.54 & 0.50 & 0.47 & 0.41 \\
\hline \multicolumn{6}{|l|}{ Cd-cov. $\mathrm{BF}_{3}$} \\
\hline Calculated & $1.95(-5)$ & $8.54(-6)$ & $3.56(-6)$ & $1.45(-6)$ & $6.07(-7)$ \\
\hline Measured & $2.85(-5)$ & $1.12(-5)$ & $4.85(-6)$ & $1.92(-6)$ & $7.34(-7)$ \\
\hline $\mathrm{C} / \mathrm{E}$ & 0.68 & 0.76 & 0.73 & 0.76 & 0.83 \\
\hline \multicolumn{6}{|l|}{ 3-in. $B^{c}$} \\
\hline Calculated & $2.50(-4)$ & $1.02(-4)$ & $4.02(-5)$ & $1.54(-5)$ & $6.08(-6)$ \\
\hline Measured & $3.12(-4)$ & $1.14(-4)$ & $4.83(-5)$ & $1.76(-5)$ & $6.60(-6)$ \\
\hline $\mathrm{C} / \mathrm{E}$ & 0.80 & 0.89 & 0.83 & 0.88 & 0.92 \\
\hline \multicolumn{6}{|l|}{ 5-in. BB } \\
\hline Calculated & $4.24(-4)$ & $1.59(-4)$ & $5.85(-5)$ & $2.12(-5)$ & $7.88(-6)$ \\
\hline Measured & $5.40(-4)$ & $1.82(-4)$ & $7.04(-5)$ & $2.51(-5)$ & $8.50(-6)$ \\
\hline $\mathrm{C} / \mathrm{E}$ & 0.79 & 0.87 & 0.83 & 0.84 & 0.93 \\
\hline \multicolumn{6}{|l|}{ 8-in. BB } \\
\hline Calculated & $1.71(-4)$ & $5.98(-5)$ & $2.08(-5)$ & $7.21(-6)$ & $2.59(-6)$ \\
\hline Measured & $2.11(-4)$ & $6.60(-5)$ & $2.40(-5)$ & $8.20(-6)$ & $2.66(-6)$ \\
\hline $\mathrm{C} / \mathrm{E}$ & 0.81 & 0.91 & 0.87 & 0.88 & 0.97 \\
\hline \multicolumn{6}{|l|}{ 10-in. BB } \\
\hline Calculated & $6.93(-5)$ & $2.35(-5)$ & $8.01(-6)$ & $2.75(-6)$ & $9.83(-7)$ \\
\hline Measured & $8.82(-5)$ & $2.48(-5)$ & $9.34(-6)$ & $3.12(-6)$ & $1.04(-6)$ \\
\hline$C / E$ & 0.79 & 0.95 & 0.86 & 0.88 & 0.95 \\
\hline
\end{tabular}

Read as $3.28 \times 10^{-5}$.

${ }^{b}$ Calculation-to-experiment ratio.

'Bonner ball. 


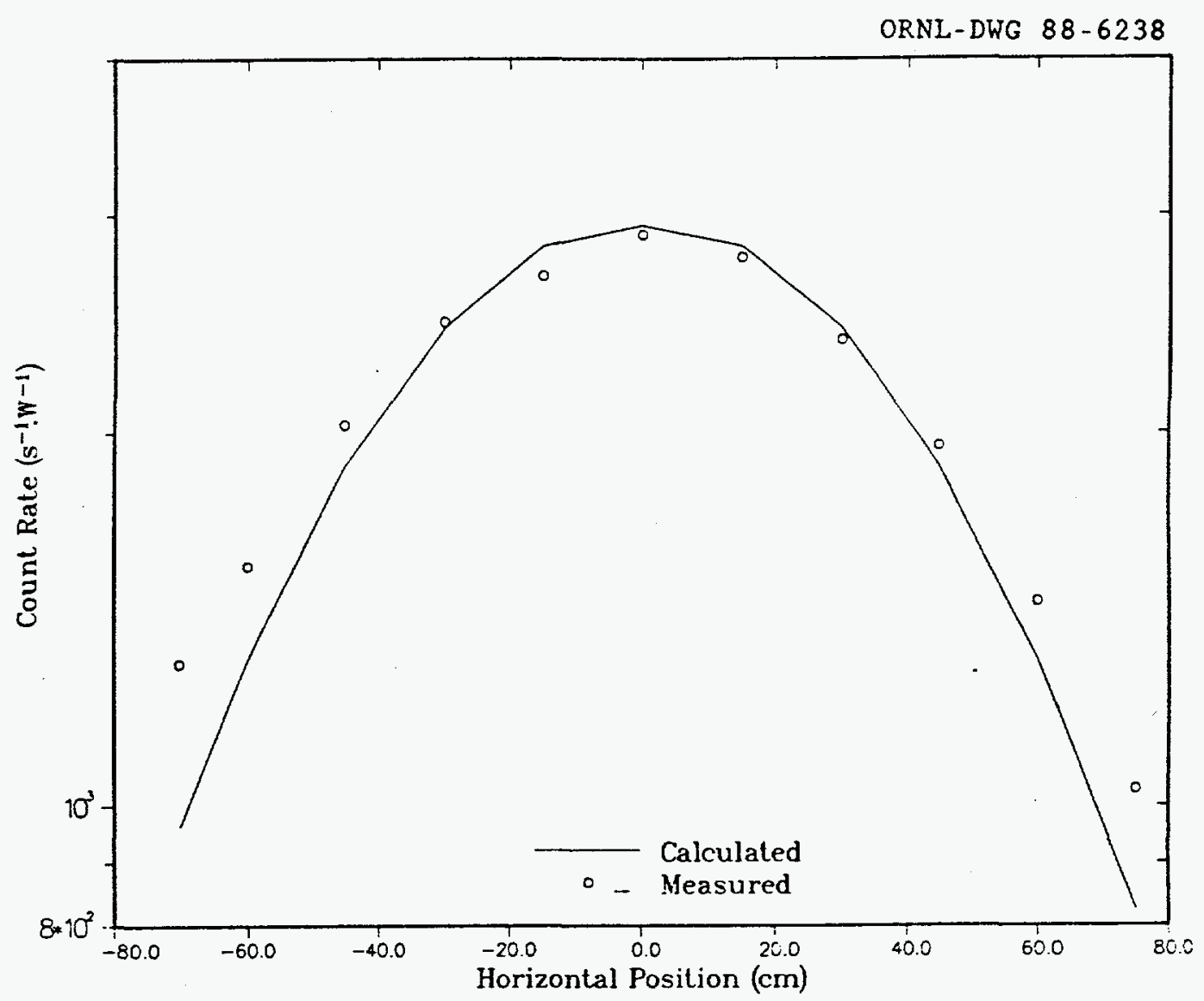

Fig. 9. Comparison of calculated and measured 5-in. Bonner ball count rates for a horizontal traverse $30 \mathrm{~cm}$ behind Configuration I.A (C/E range: 0.74-1.06) 


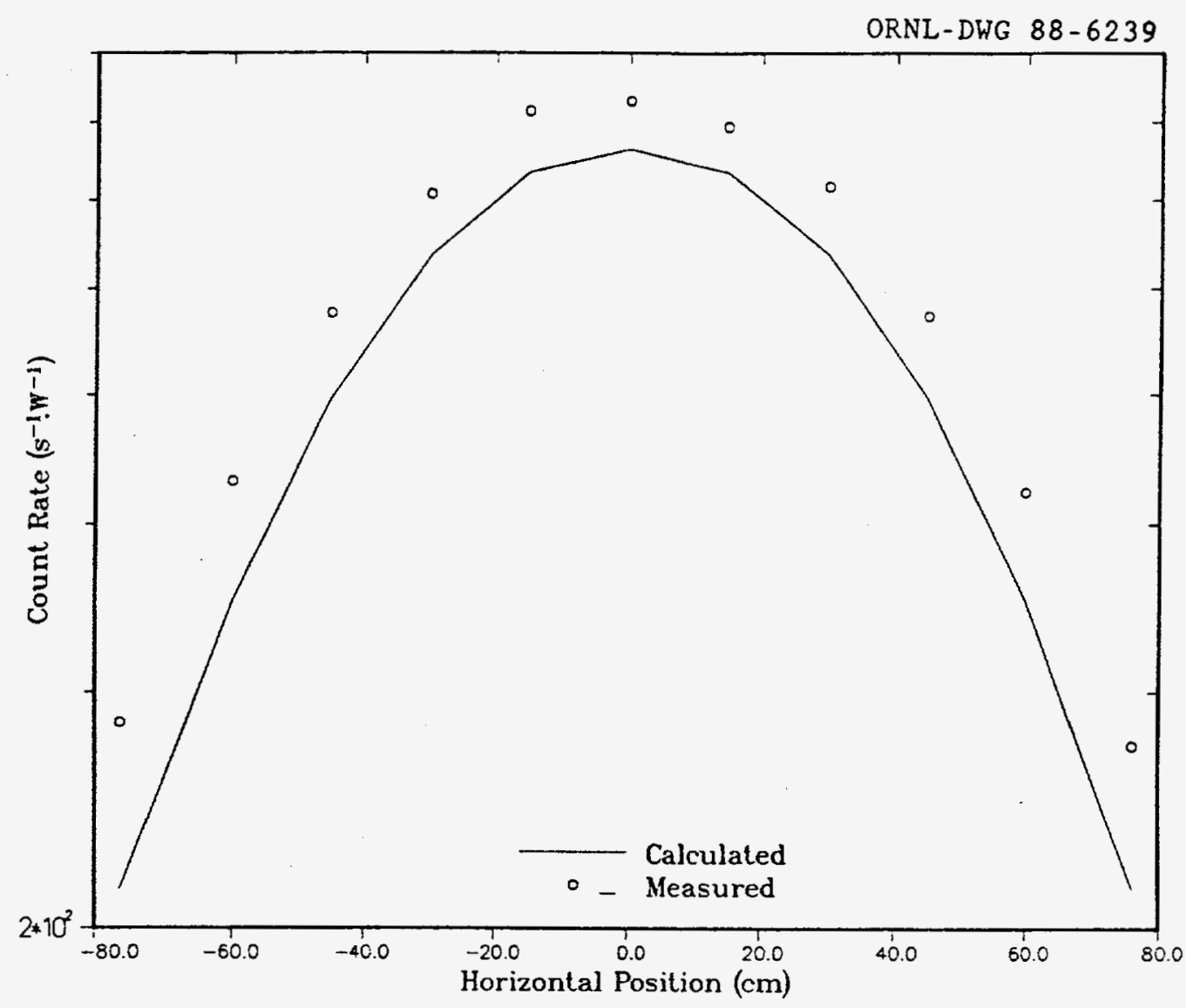

Fig. 10. Comparison of calculated and measured 5-in. Bonner ball count rates for a horizontal traverse $30 \mathrm{~cm}$ behind Configuration II.A (C/E range: $0.75-0.92)$ 


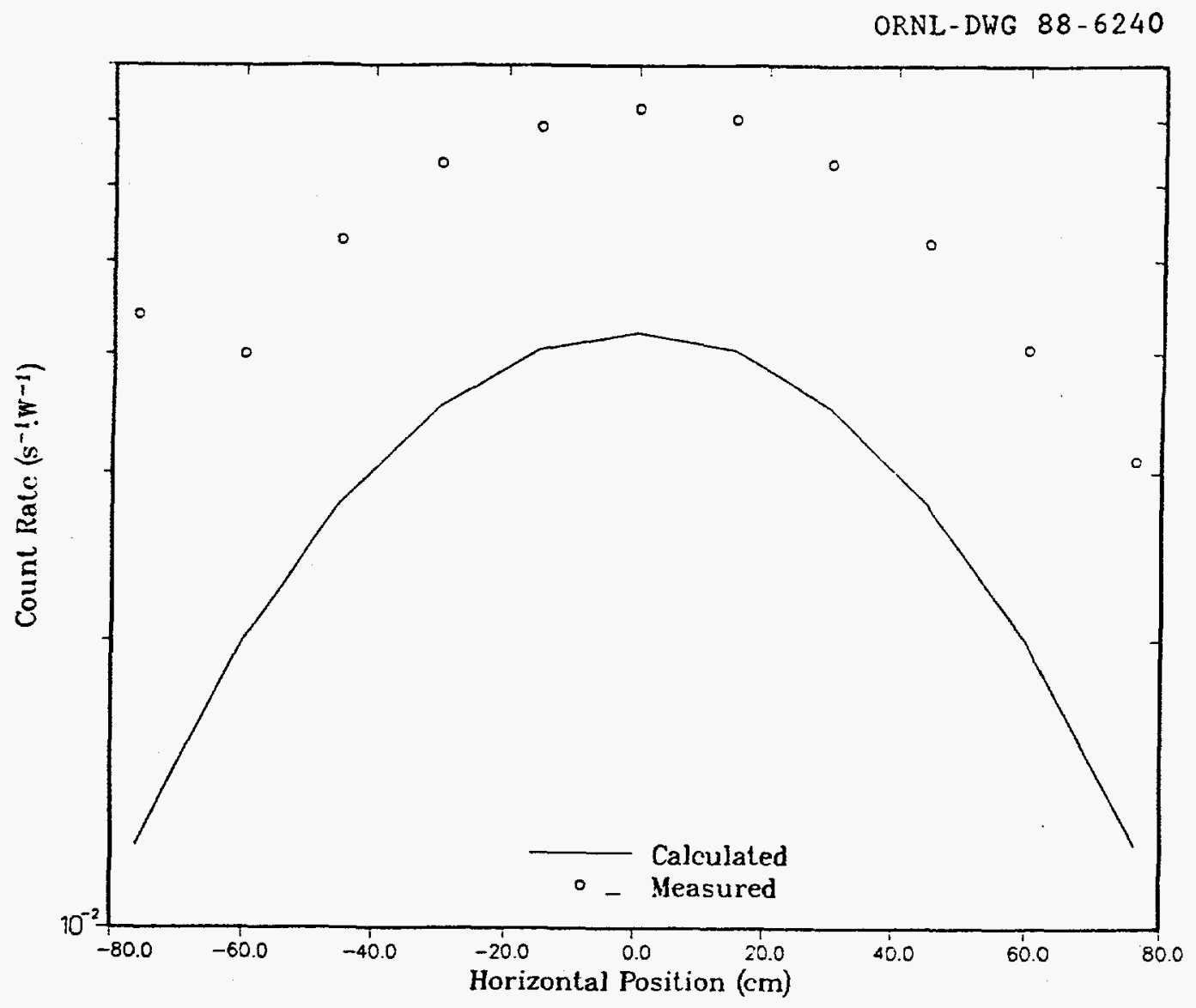

Fig. 11. Comparison of calculated and measured 5 -in. Bonner ball count rates for a horizontal traverse $30 \mathrm{~cm}$ behind Configuration II.D (C/E range: $0.28-0.58$ ) 
ORNL-DWG $88-6241$

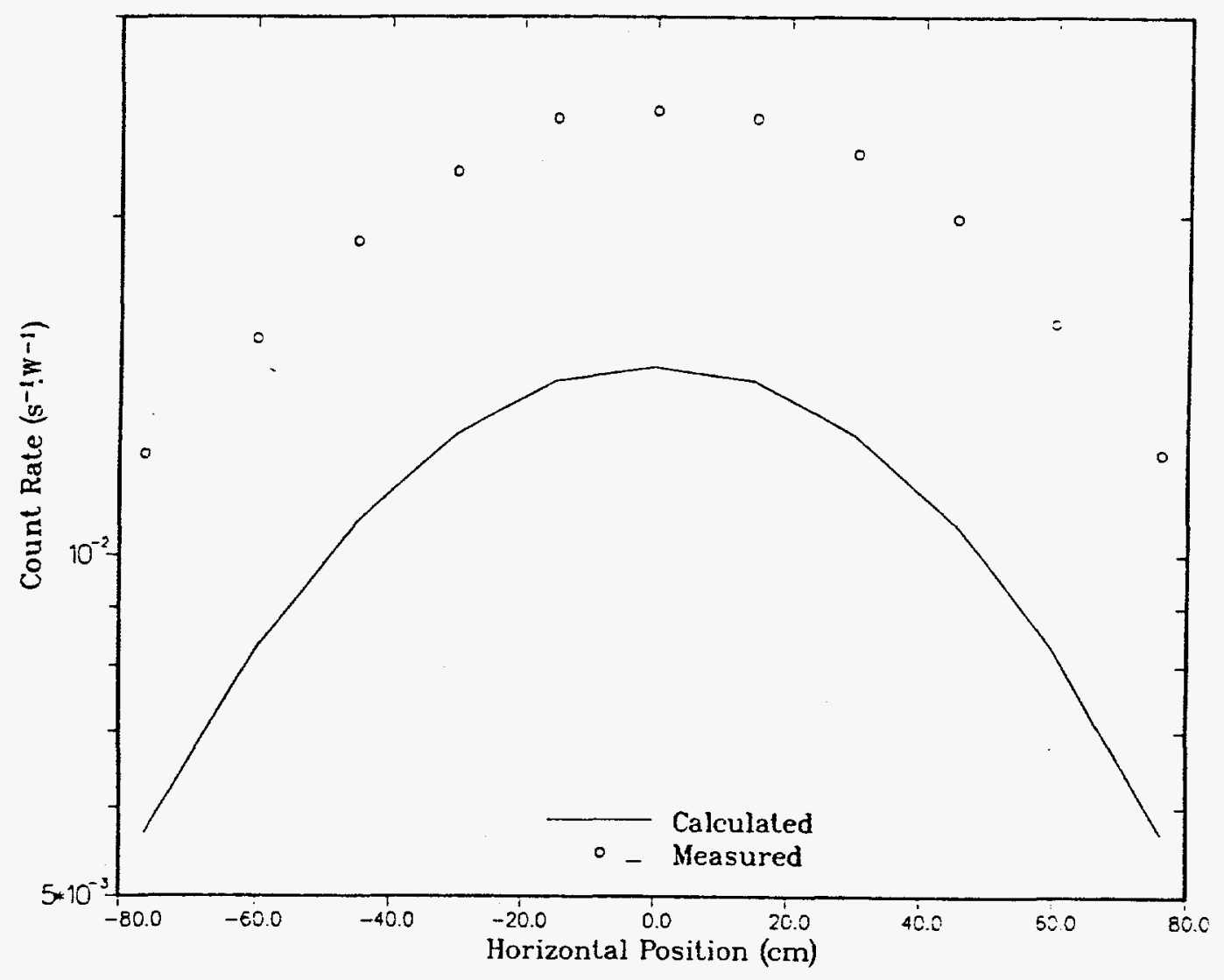

Fig. 12. Comparison of calculated and measured 5-in. Bonner ball count rates for a horizontal traverse $30 \mathrm{~cm}$ behind Configuration II.E (C/E range: 0.46-0.59) 


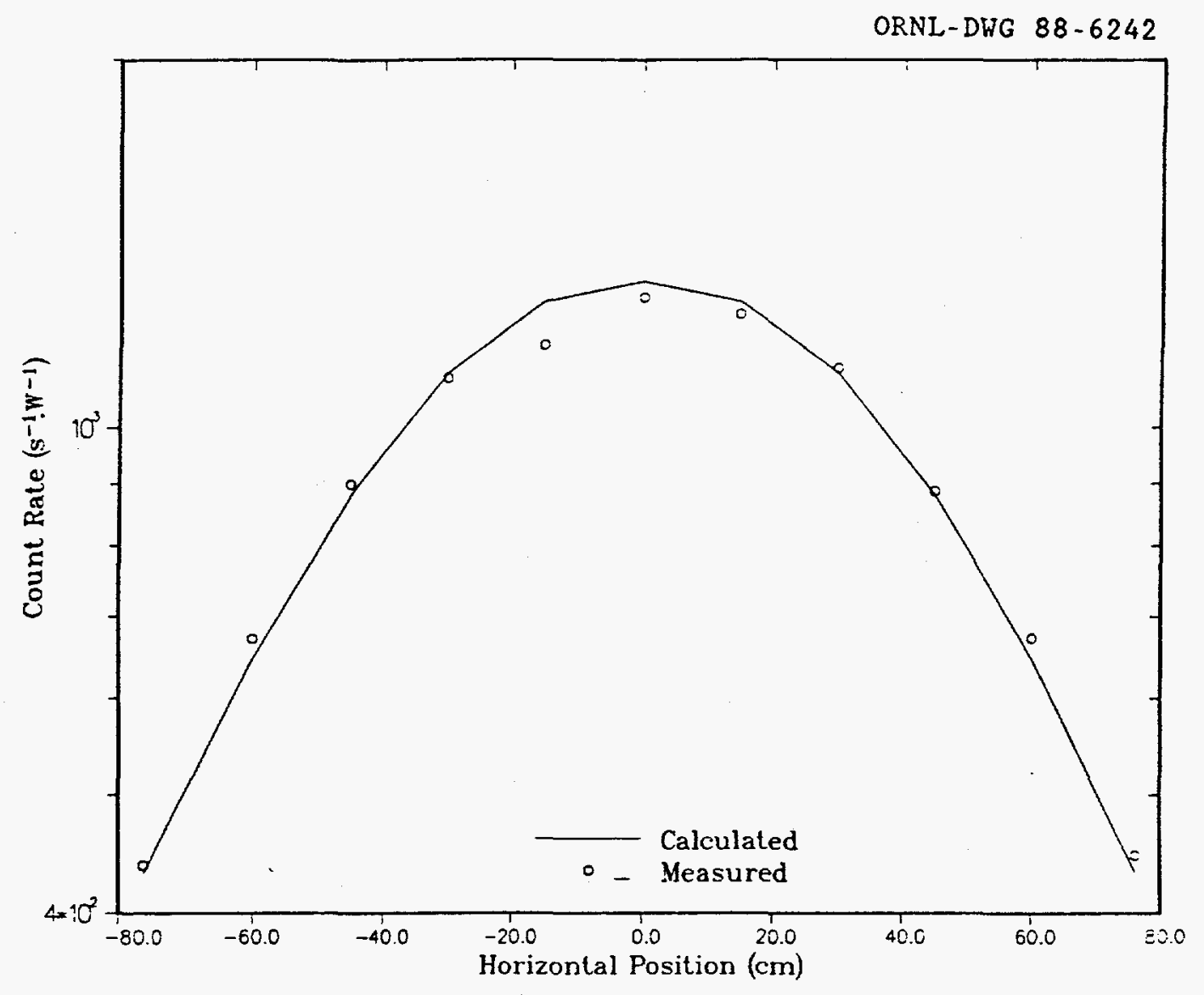

Fig. 13. Comparison of calculated and measured 5-in. Bonner ball count rates for a horizontal traverse $30 \mathrm{~cm}$ behind Configuration III.A (C/E range: 0.96-1.09) 


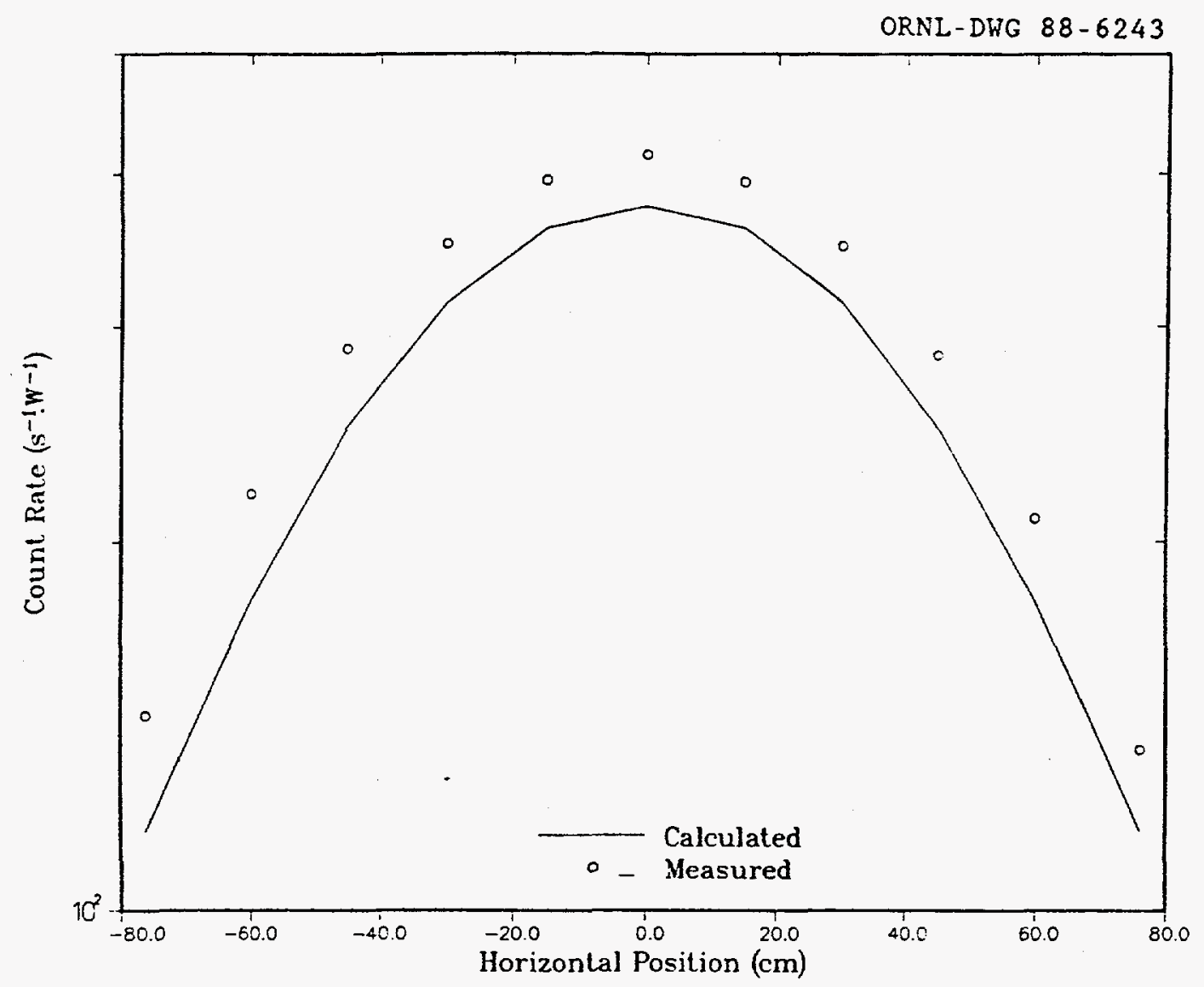

Fig. 14. Comparison of calculated and measured 5-in. Bonner ball count rates for a horizontal traverse $30 \mathrm{~cm}$ behind Configuration III.B (C/E range: $0.81-0.94$ ) 


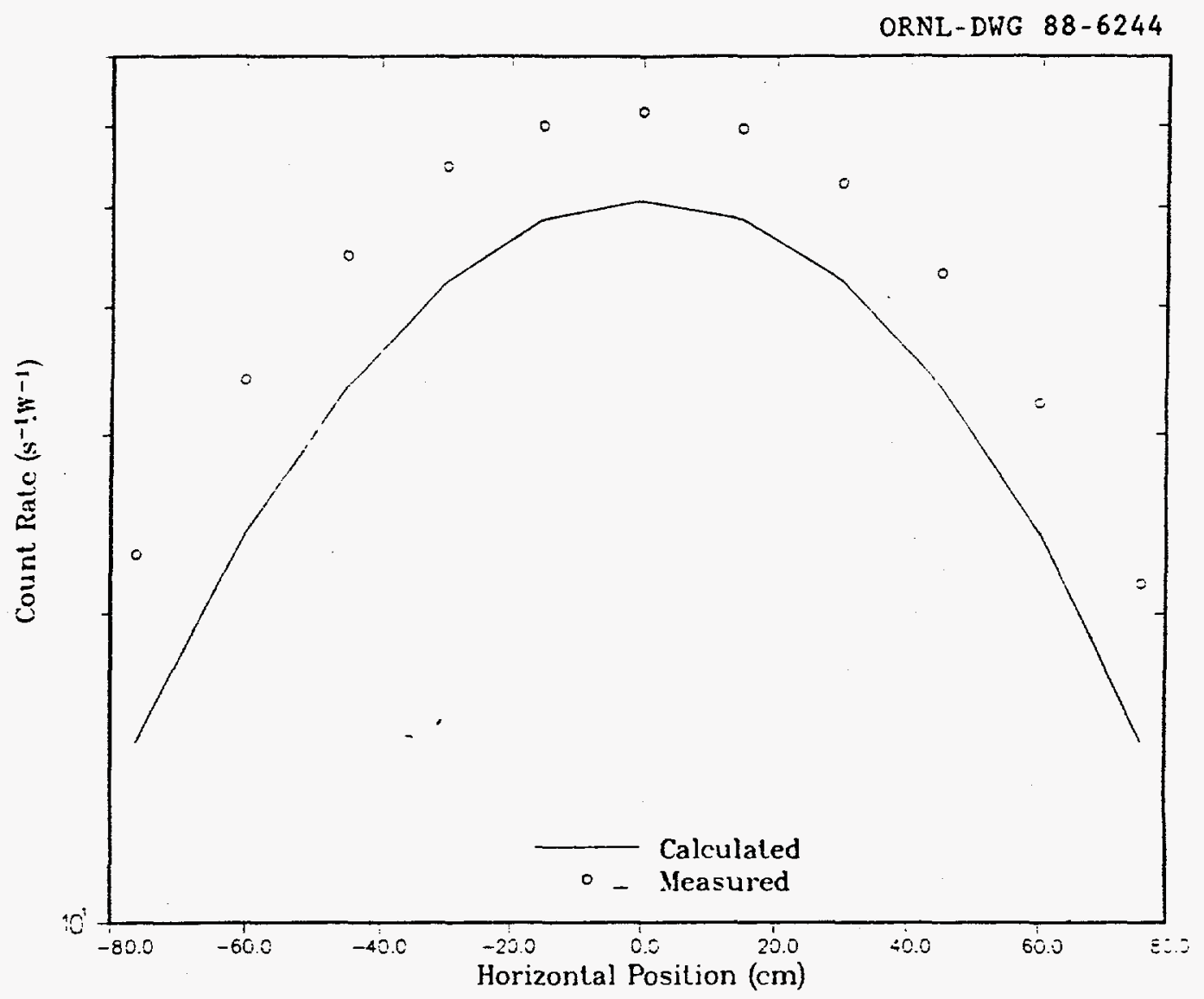

Fig. 15. Comparison of calculated and measured 5-in. Bonner ball count rates for a horizontal traverse $30 \mathrm{~cm}$ behind Configuration III.C (C/E range: $0.77-0.89$ ) 


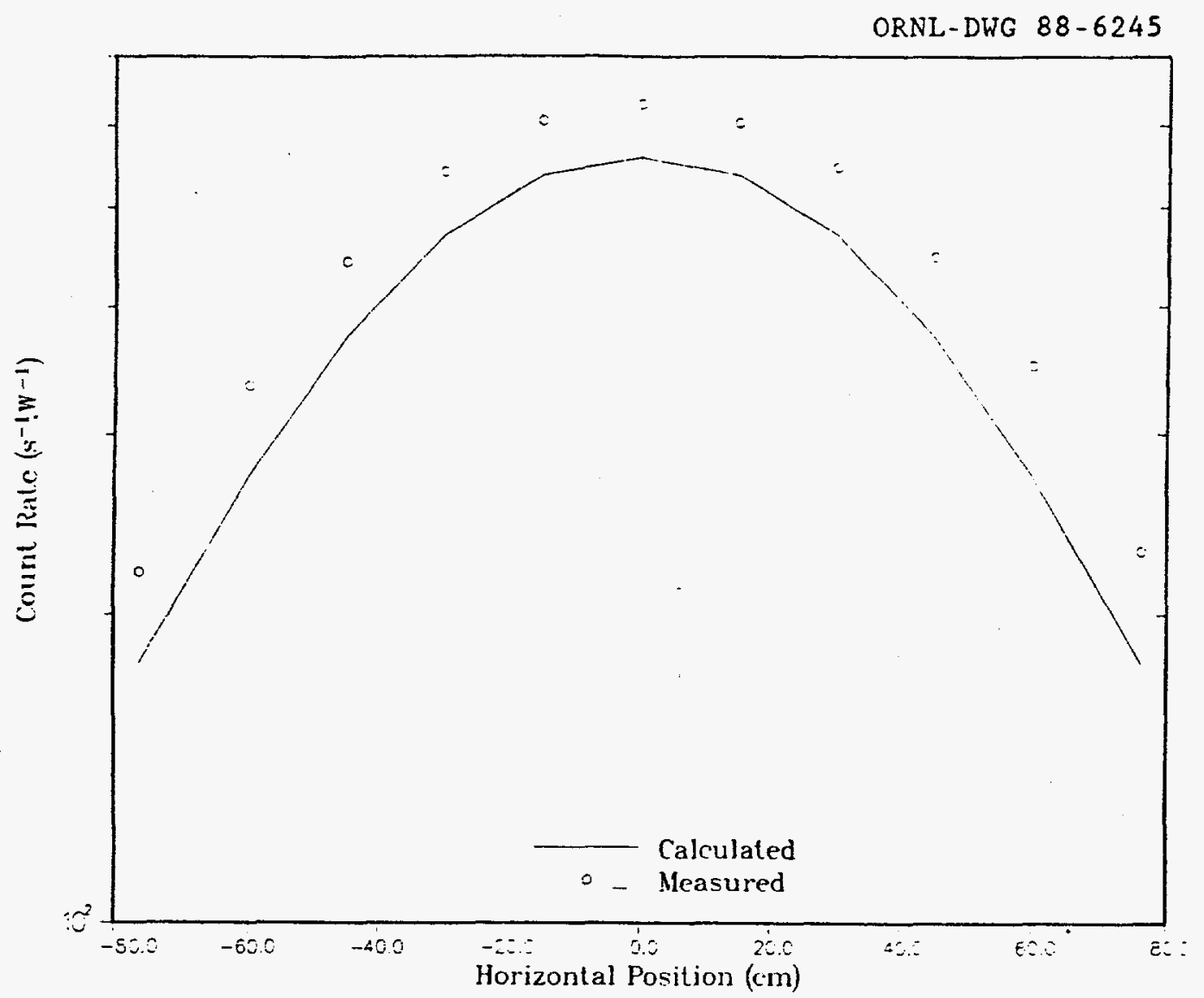

Fig. 16. Comparison of calculated and measured 5-in. Bonner ball count rates for a horizontal traverse $30 \mathrm{~cm}$ behind Configuration III.E (C/E range: $0.66-0.82$ ) 
ORNL-DWG $88-6246$

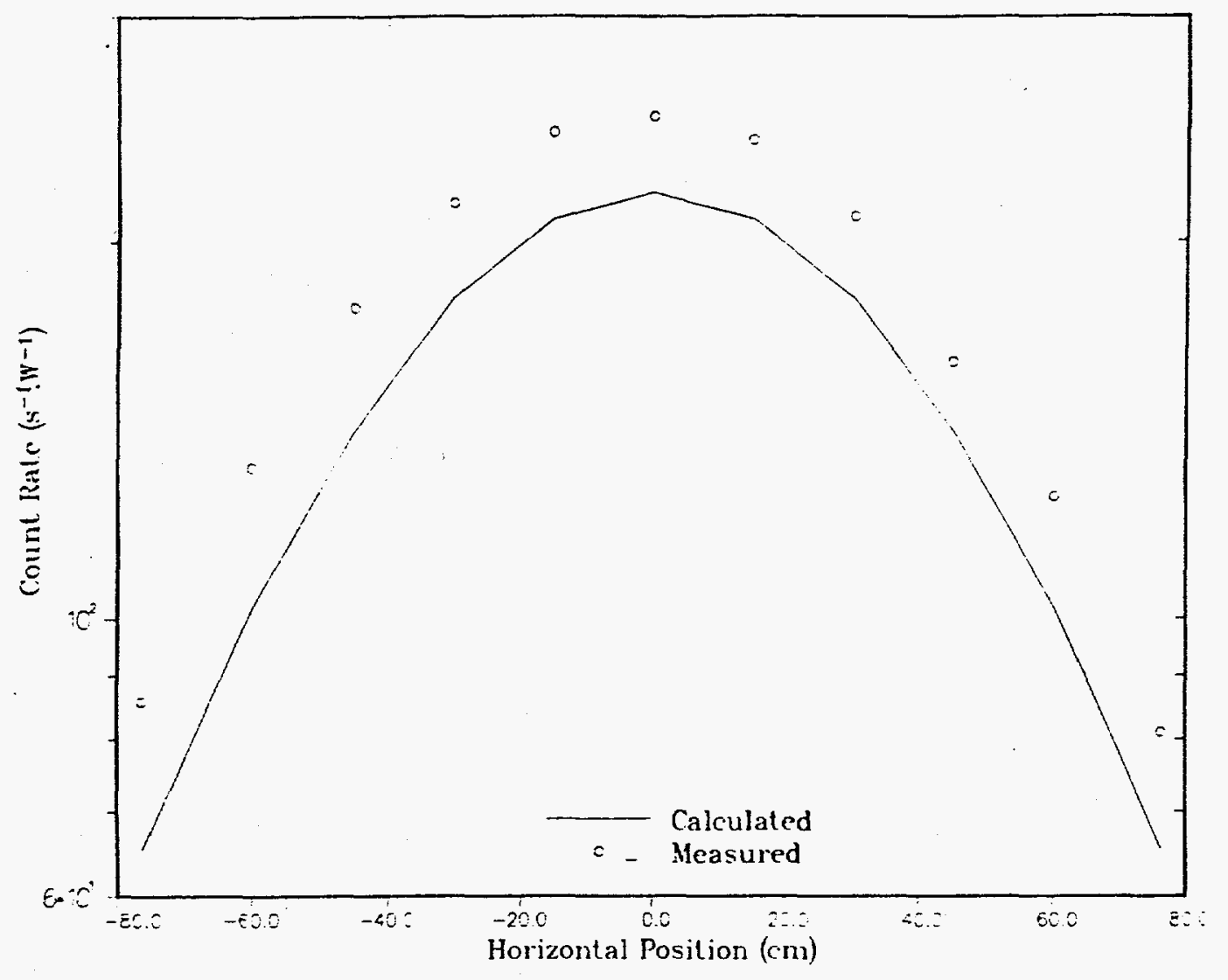

Fig. 17. Comparison of calculated and measured 5-in. Bonner ball count rates for a horizontal traverse $30 \mathrm{~cm}$ behind Configuration IV.A (C/E range: 0.76-0.87) 


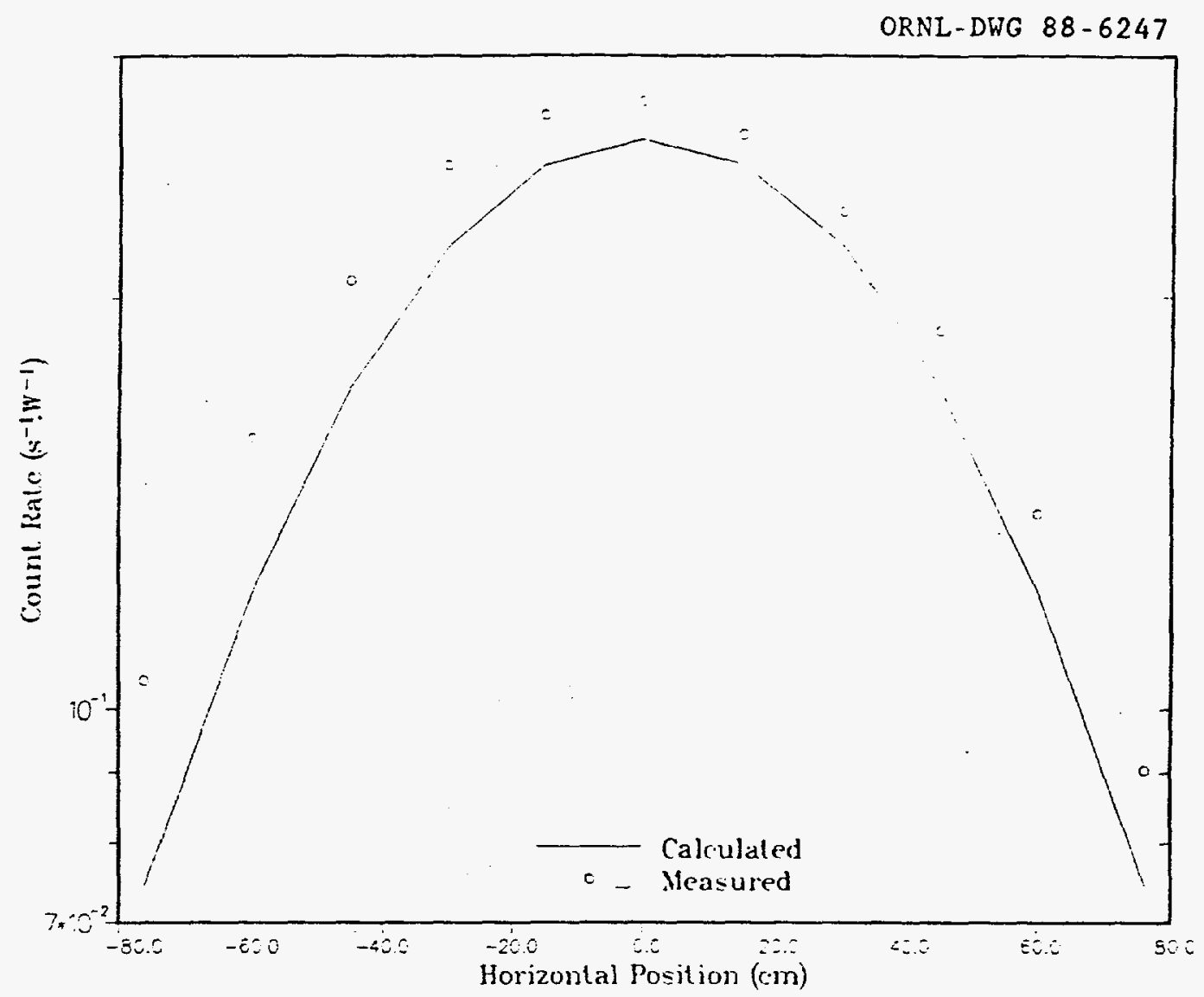

Fig. 18. Comparison of calculated and measured 5 -in. Bonner ball count rates for a horizontal traverse $30 \mathrm{~cm}$ behind Configuration IV.C (C/E range: $0.71-0.95$ ) 


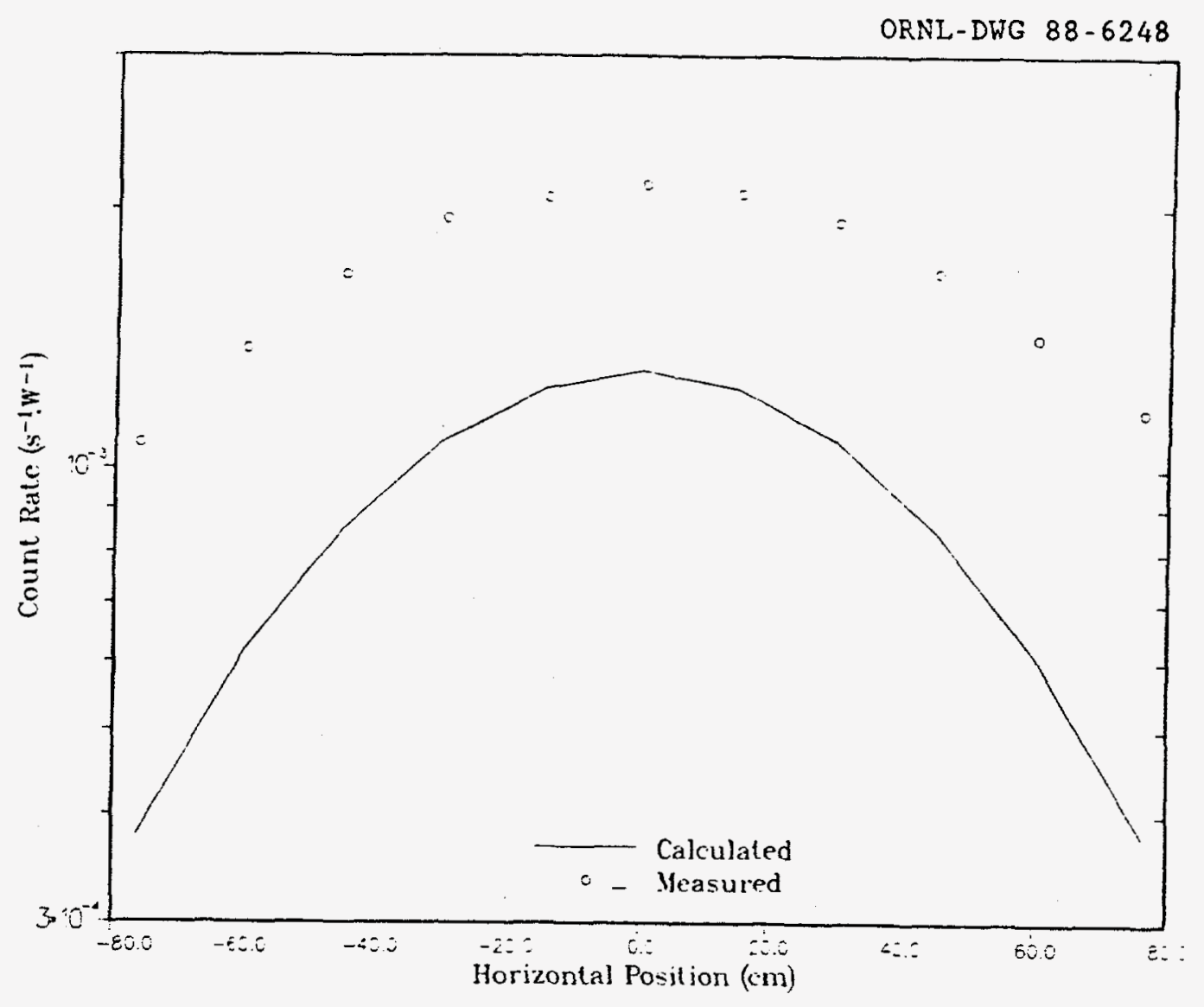

Fig. 19. Comparison of calculated and measured 5 -in. Bonner ball count rates for a horizontal traverse $30 \mathrm{~cm}$ behind Configuration IV.G (C/E range: $0.32-0.61$ ) 


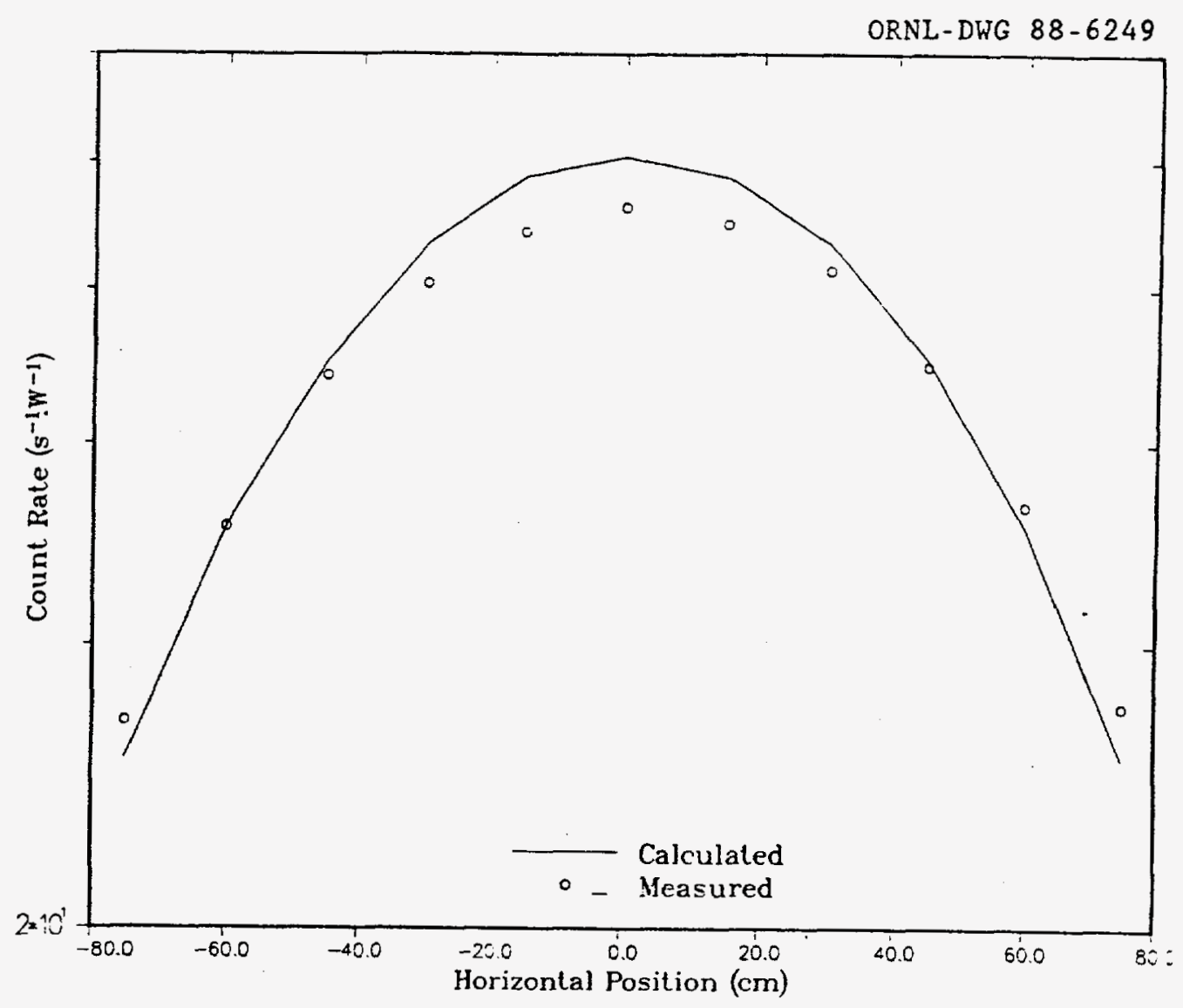

Fig. 20. Comparison of calculated and measured 5-in. Bonner ball count rates for a horizontal traverse $30 \mathrm{~cm}$ behind Configuration V.A (C/E range: 0.93-1.08) 


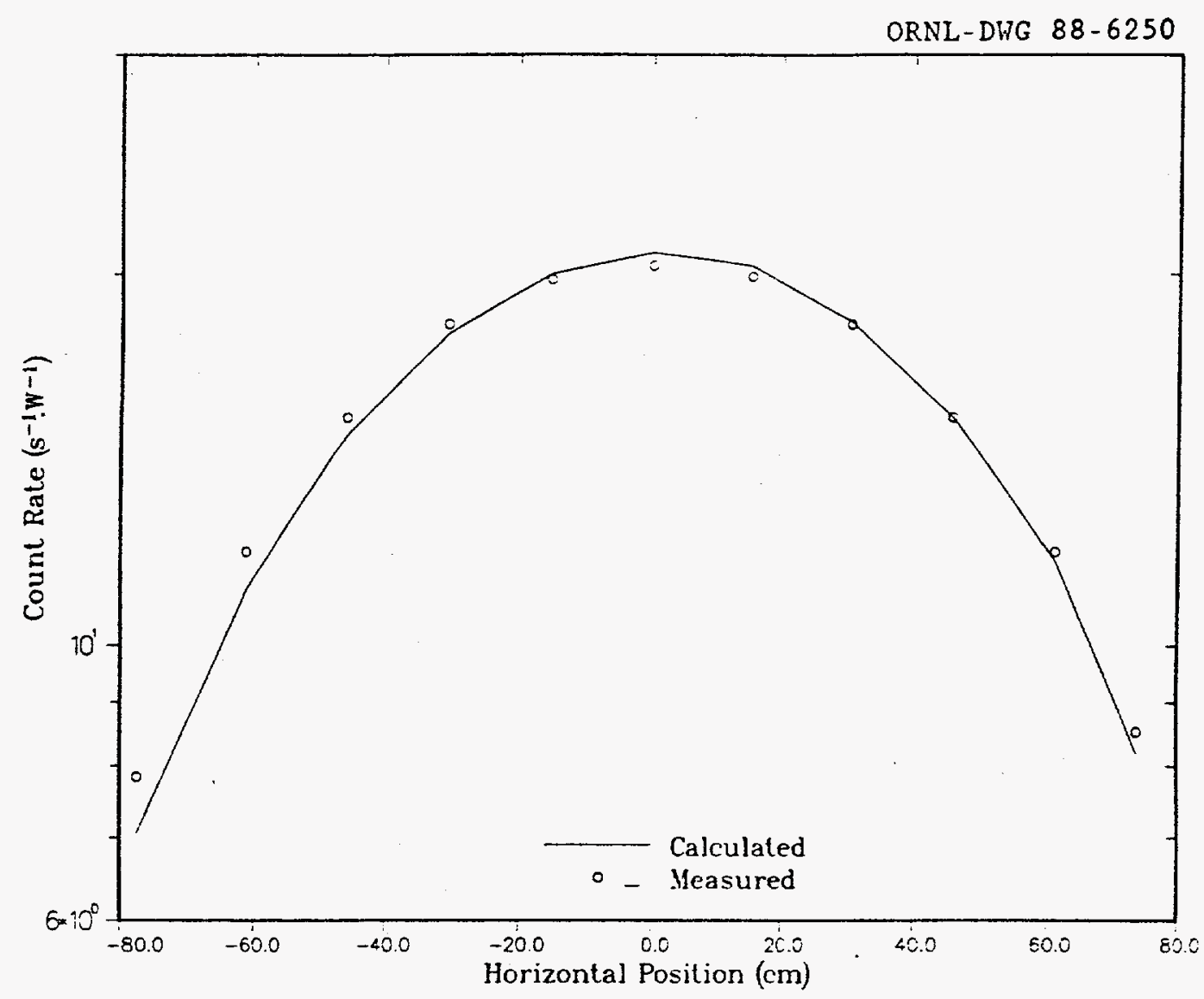

Fig. 21. Comparison of calculated and measured 5-in. Bonner ball count rates for a horizontal traverse $30 \mathrm{~cm}$ behind Configuration V.B (C/E range: 0.90-1.02) 


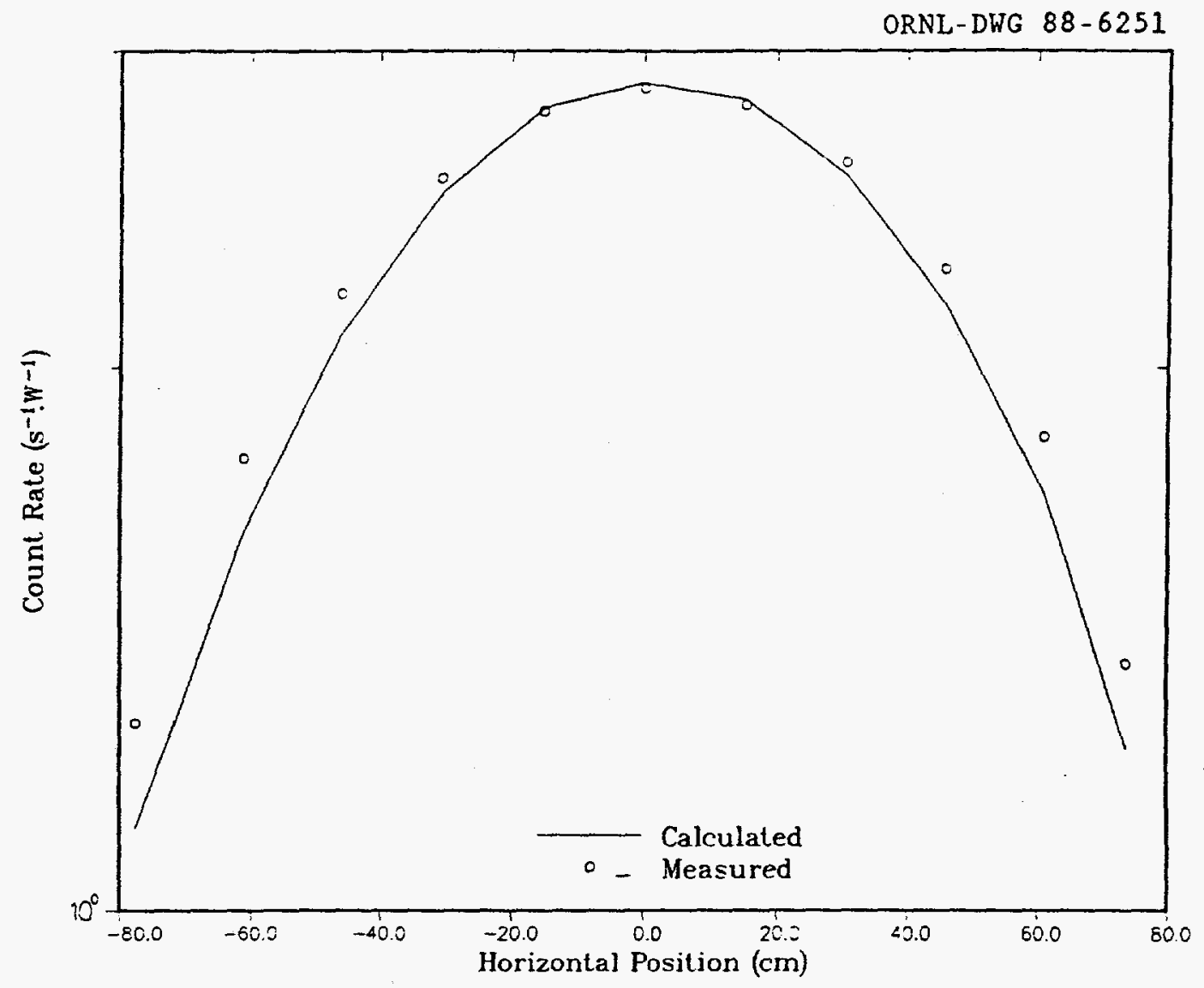

Fig. 22. Comparison of calculated and measured 5-in. Bonner ball count rates for a horizontal traverse $30 \mathrm{~cm}$ behind Configuration V.D (C/E range: $0.87-1.01$ ) 


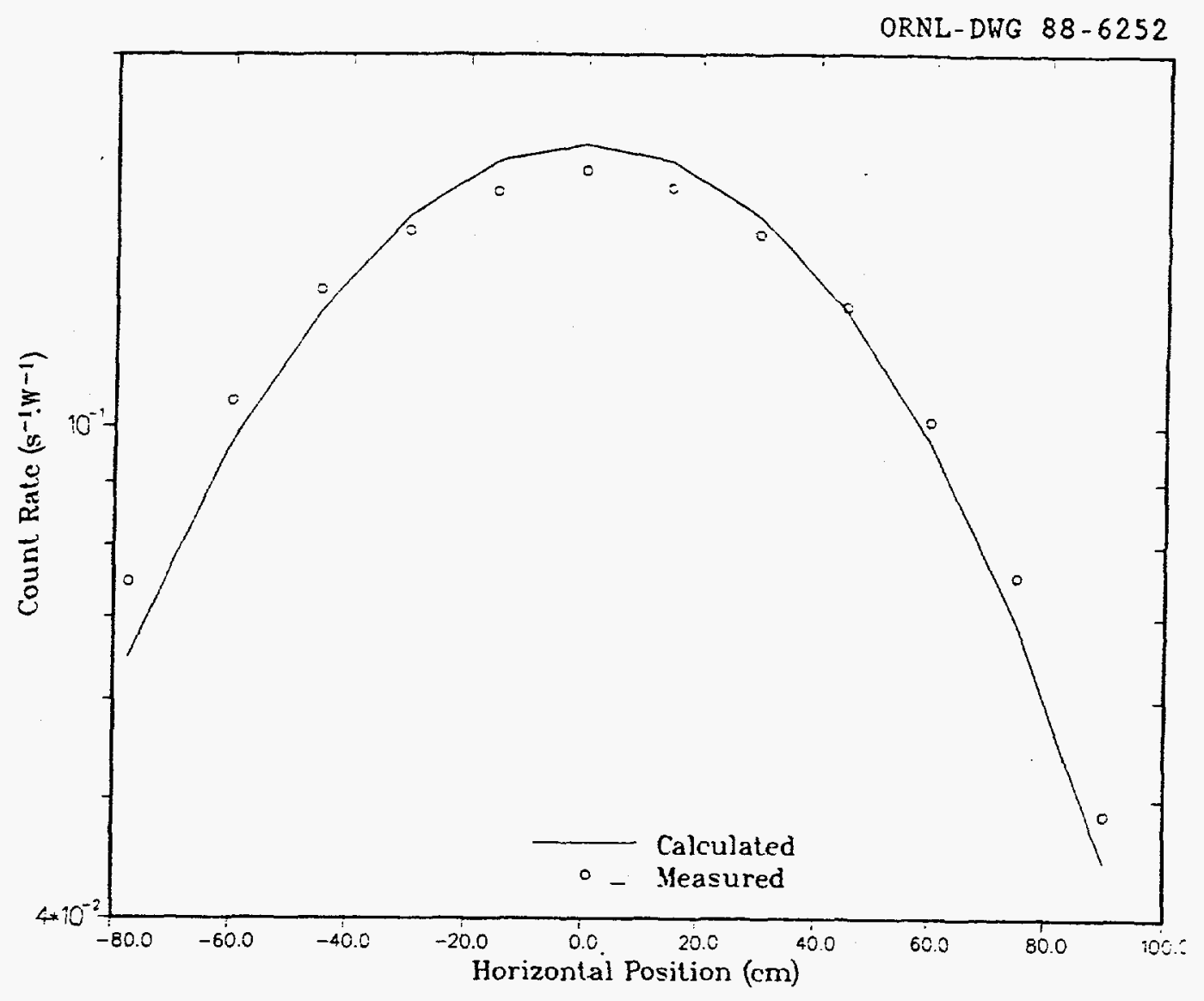

Fig. 23. Comparison of calculated and measured 5-in. Bonner ball count rates for a horizontal traverse $30 \mathrm{~cm}$ behind Configuration V.G (C/E range: $0.87-1.06$ ) 


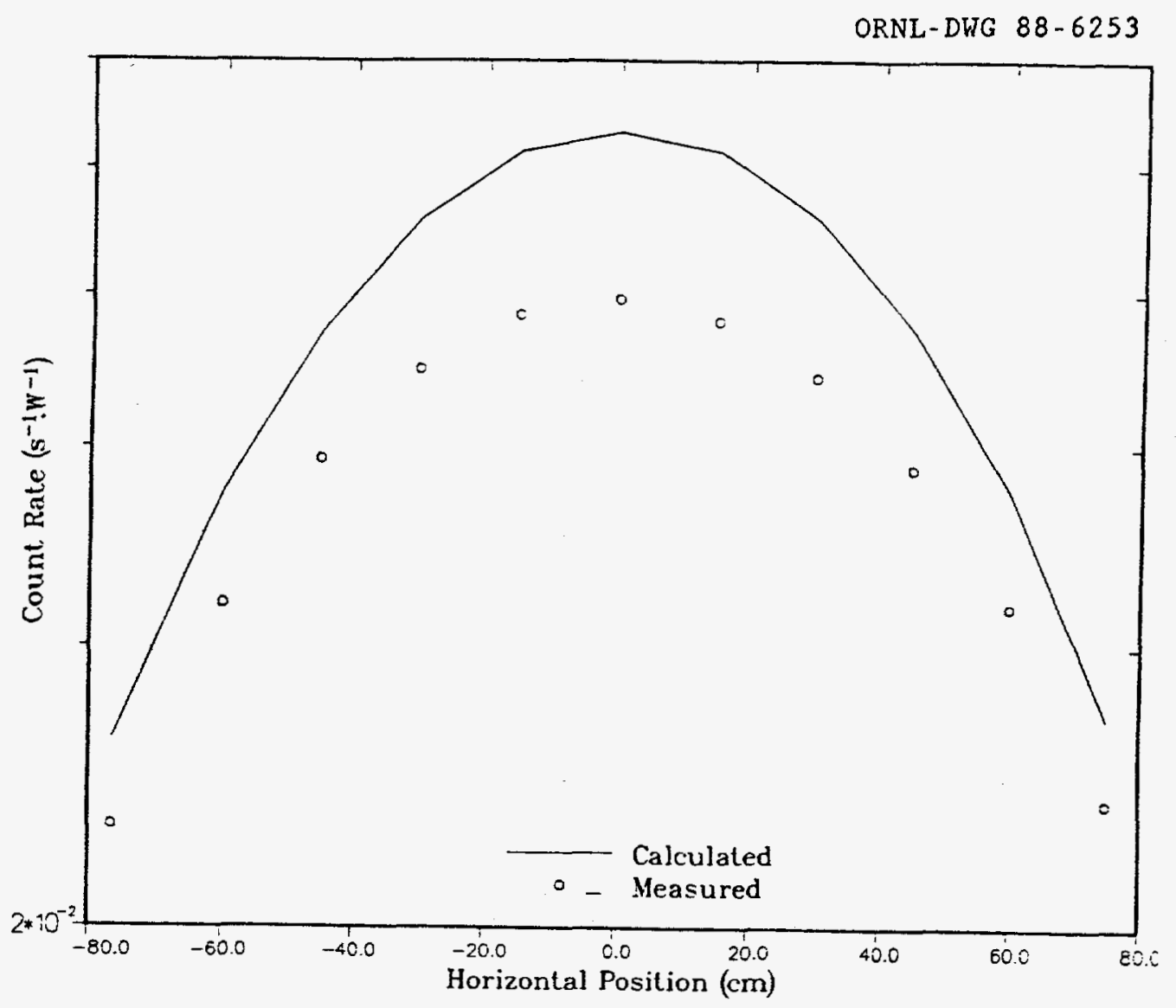

Fig. 24. Comparison of calculated and measured 5 -in. Bonner ball count rates for a horizontal traverse $30 \mathrm{~cm}$ behind Configuration V.J (C/E range: 1.13-1.2S) 


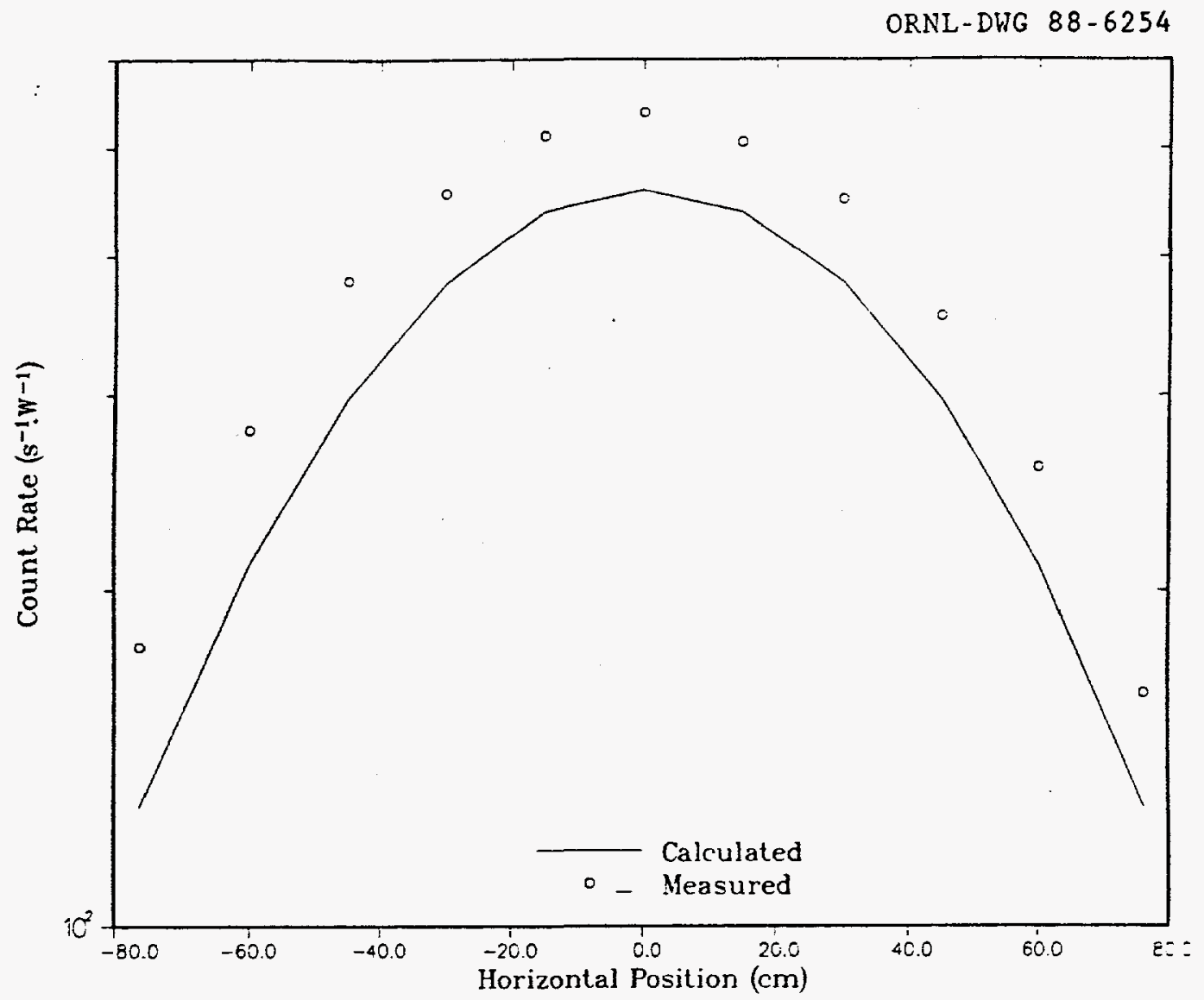

Fig. 25. Comparison of calculated and measured 5-in. Bonner ball count rates for a horizontal traverse $30 \mathrm{~cm}$ behind Configuration VI.A (C/E range: $0.72-0.87$ ) 


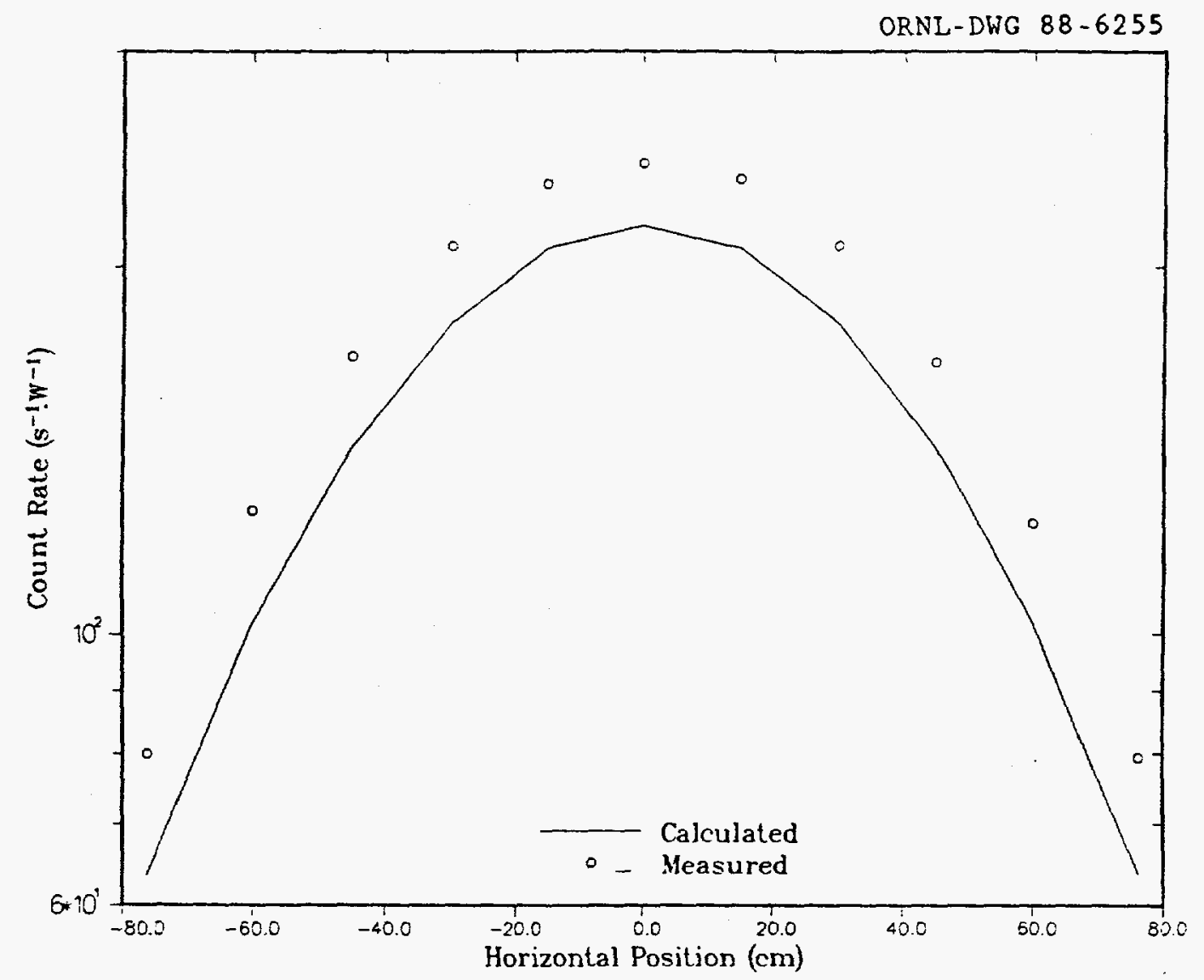

Fig. 26. Comparison of calculated and measured 5-in. Bonner ball count rates for a horizontal traverse $30 \mathrm{~cm}$ behind Configuration VI.B (C/E range: 0.79-0.89) 


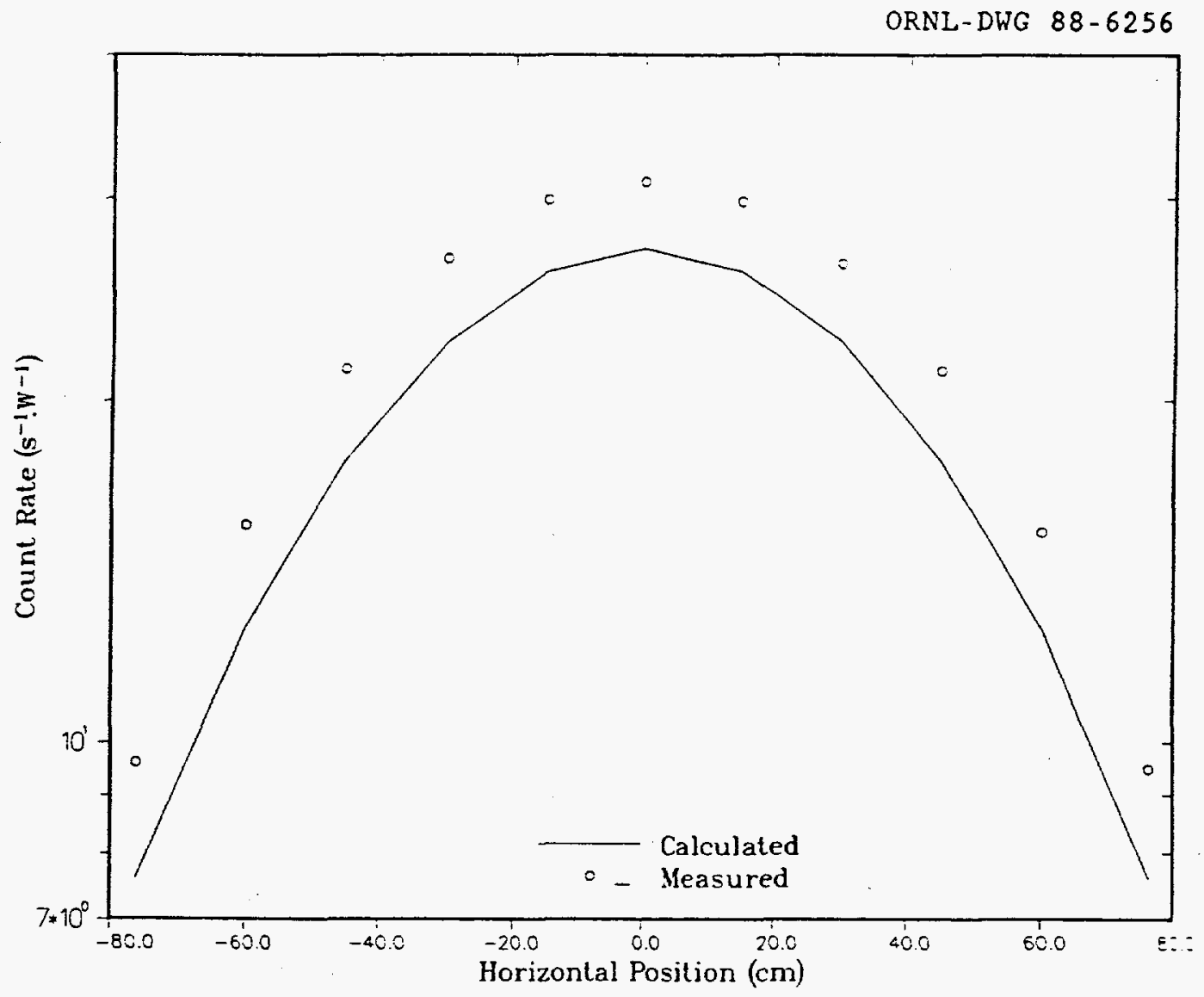

Fig. 27. Comparison of calculated and measured 5-in. Bonner ball count rates for a horizontal traverse $30 \mathrm{~cm}$ behind Configuration VI.D (C/E range: 0.79-0.87) 


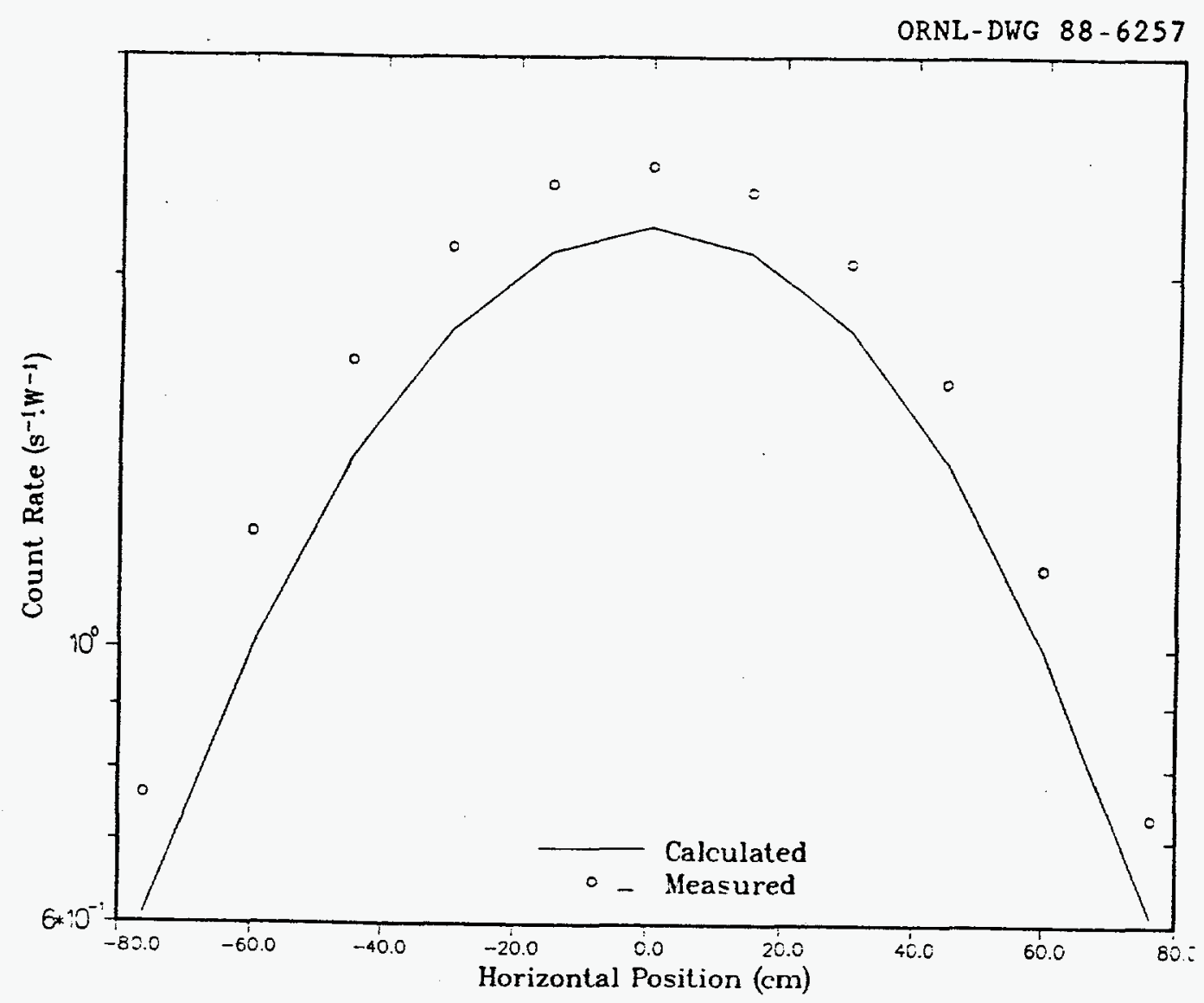

Fig. 28. Comparison of calculated and measured 5 -in. Bonner ball count rates for a horizontal traverse $30 \mathrm{~cm}$ behind Configuration VI.F (C/E range: $0.80-0.89$ ) 


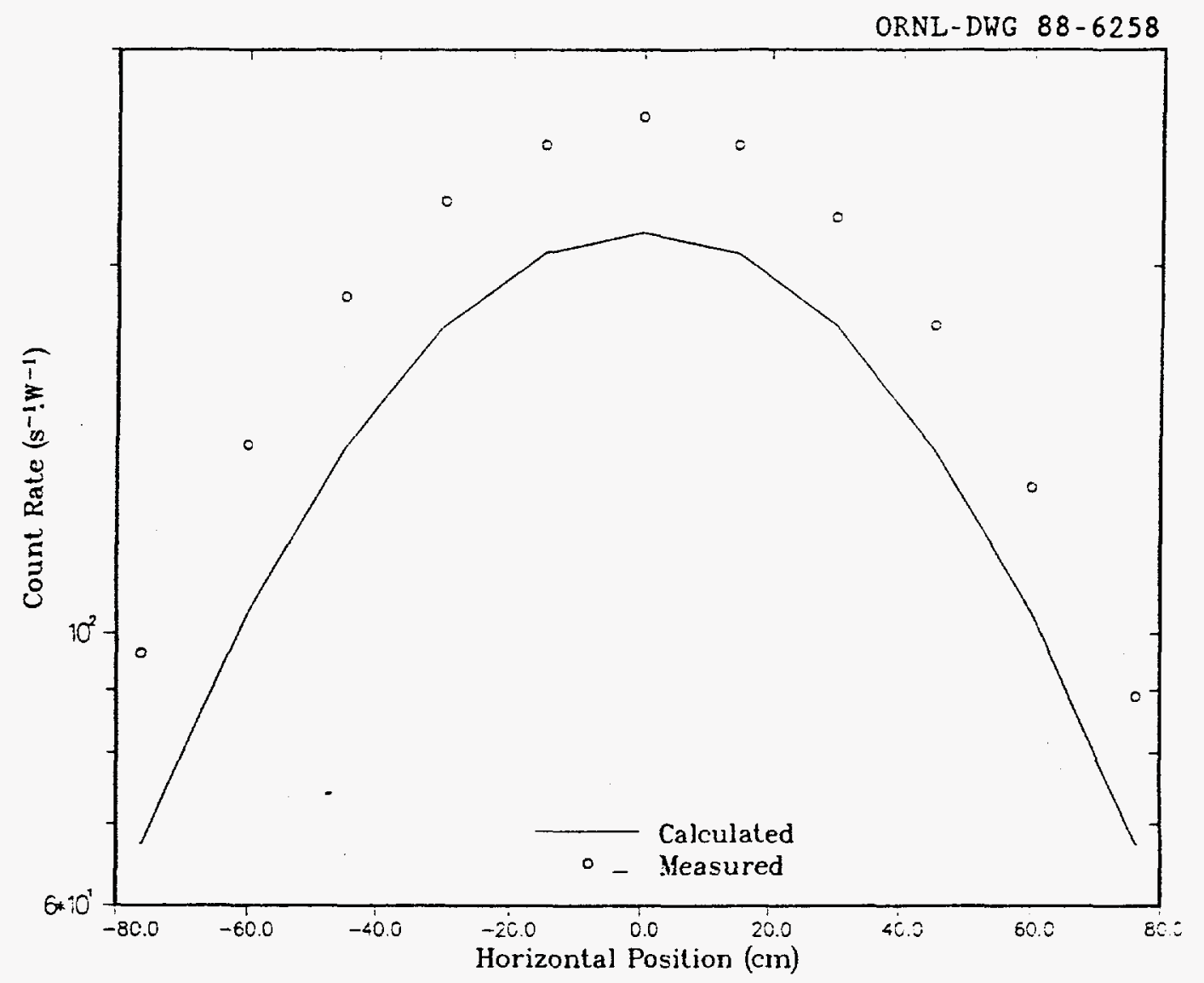

Fig. 29. Comparison of calculated and measured 5-in. Bonner ball count rates for a horizontal traverse $30 \mathrm{~cm}$ behind Configuration VII.A (C/E range: $0.70-0.82$ ) 


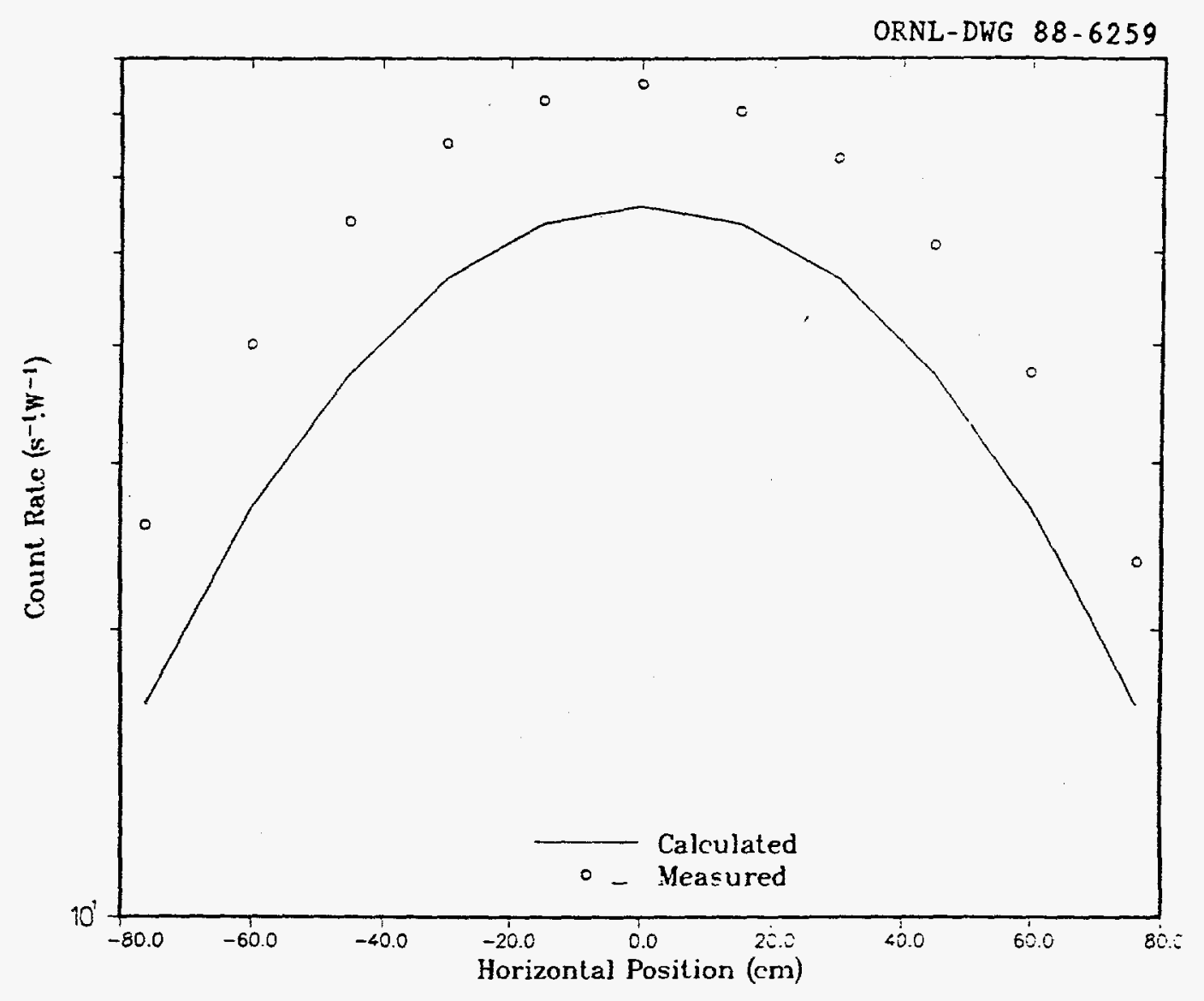

Fig. 30. Comparison of calculated and measured 5 -in. Bonner ball count rates for a horizontal traverse $30 \mathrm{~cm}$ behind Configuration VII.B (C/E range: $0.65-0.76$ ) 


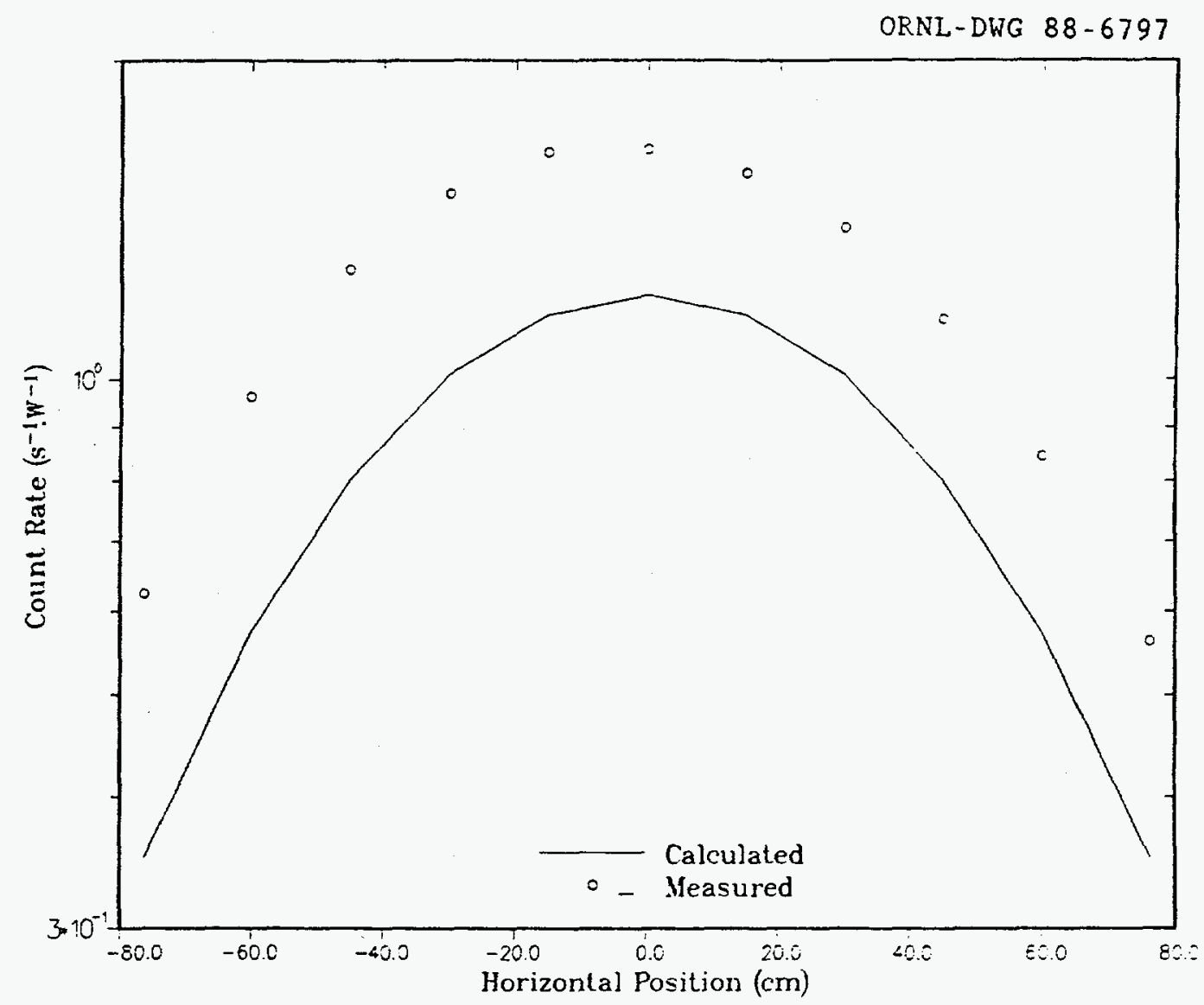

Fig. 31. Comparison of calculated and measured 5-in. Bonner ball count rates for a horizontal traverse $30 \mathrm{~cm}$ behind Configuration VII.C (C/E range: $0.56-0.73$ ) 


\subsection{Discrepancies Between Calculated and Measured Spectra}

The group structure was analyzed as a possible cause of the discrepancy between the calculated and measured neutron spectra. ANISN 1-D spherical geometry calculations were performed for Configurations II.D $(1,2)$ and VI.F $(1,2)$ using the 61-group library and the 174-group library from which it was derived. The 61-group spectra were normalized to the DOT-IV spectra and the 174-group spectra were scaled upward by the factors used to normalize the 61 -group spectra (this factor was about 1.25 for both configurations). The two calculated spectra are compared with the measured spectra in Figs. 32 and 33 for Configurations II.D $(1,2)$ and VI.F $(1,2)$, respectively. The integrated fast-neutron flux for the 174-group calculations was found to be a factor of 1.03 higher for Configuration II.D $(1,2)$ and a factor of 1.26 higher for Configuration VI.F $(1,2)$. Thus, the group structure shows little effect for Configuration II.D $(1,2)$ but a significant improvement in radiation transmission for Configuration VI.F $(1,2)$. The C/E for Configuration II.D $(1,2)$ would increase from 0.88 to 0.91 , while that for Configuration VI.F $(1,2)$ would increase from 0.84 to 1.06 , probably due to the better representation of the carbon cross section with the fine-group structure.

\subsection{Cross-Section Sensitivities}

Because of the discrepancies between the calculated and measured detector responses, cross-section sensitivities were studied in order to determine the importance of the cross sections of various elements to calculated radiation levels. Two configurations, II.D $(1,2)$ and IV.H, were studied. Configuration II.D $(1,2)$ contained large amounts of steel and $\mathrm{B}_{4} \mathrm{C}$ while Configuration IV.H contained large amounts of steel and graphite. Sensitivities were calculated for the neutron flux in three energy ranges: $\mathrm{E}>1.1 \mathrm{MeV}, \mathrm{E}$ $>0.12 \mathrm{MeV}$, and total ( $\mathrm{E}>0.0 \mathrm{MeV}$ ). The results are shown in Table 17 for Configuration II.D(1,2) and Table 18 for Configuration IV.H. Cross sections for $\mathrm{Fe},{ }^{11} \mathrm{~B}$, $\mathrm{A}$, and ${ }^{238} \mathrm{U}$ are most important for Configuration $\Pi \mathrm{I}$. $(1,2)$. Iron shows a high-energy effect while boron shows mostly a low-energy effect. For Configuration IV.H, the most important cross sections are those for $\mathrm{Fe}, \mathrm{C}, \mathrm{Al}, \mathrm{Cr}$, and ${ }^{238} \mathrm{U}$. The only positive sensitivity shown is for ${ }^{235} \mathrm{U}$, which is responsible the fission neutron source in the blankets. 
CENL/DHE B7.12763

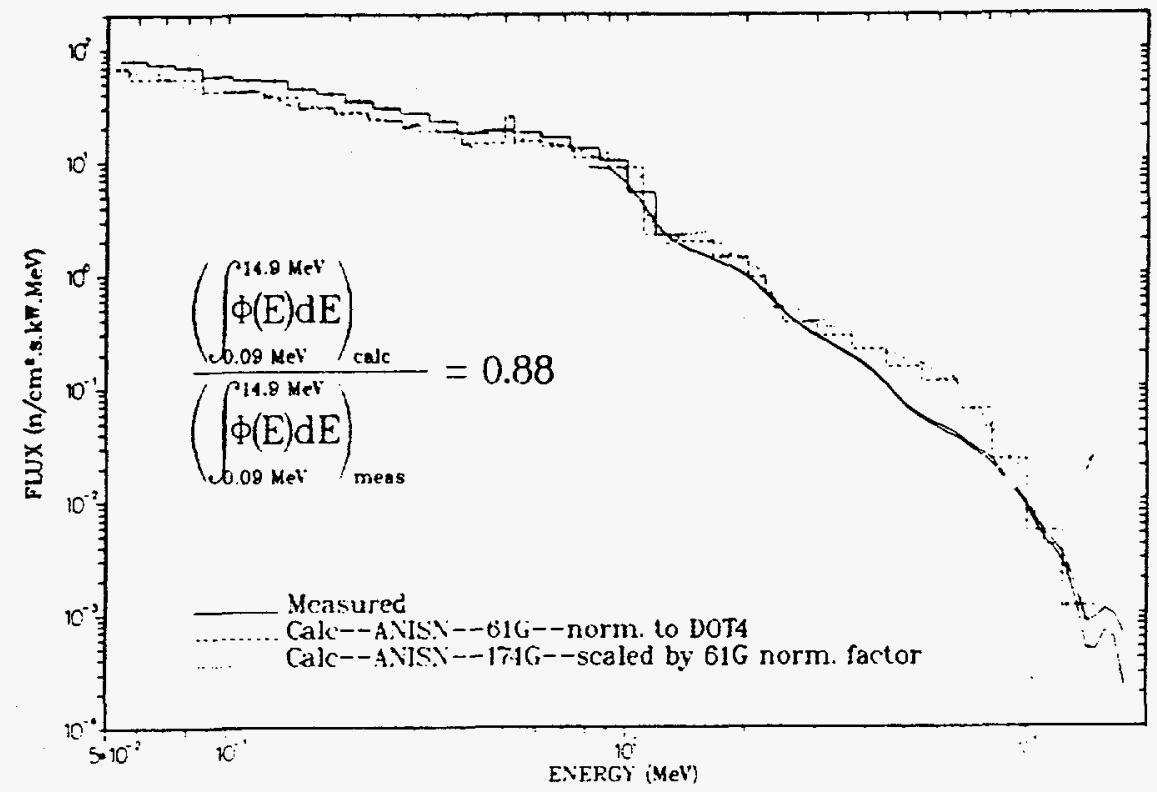

Fig. 32. Comparison of calculated 61-broad-group and 174-fine-group fast-neutron spectra with the measured spectrum on centerline $47.3 \mathrm{~cm}$ behind Configuration II.D

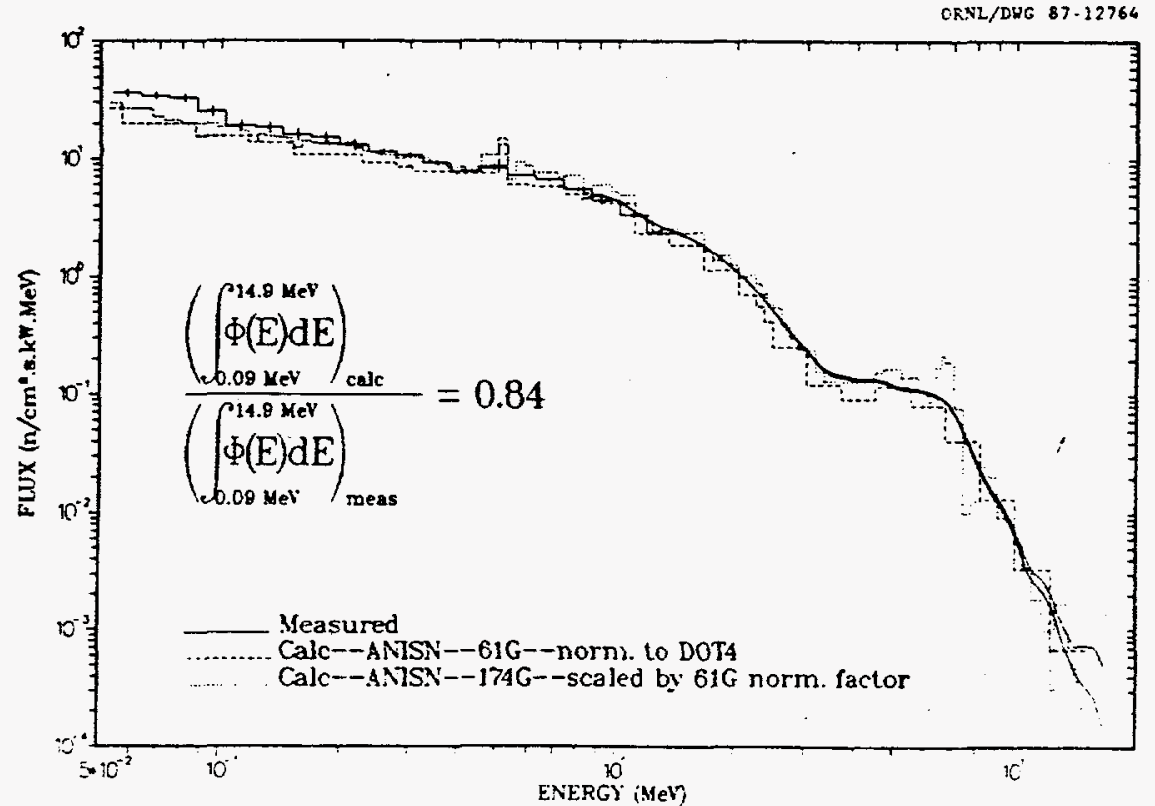

Fig. 33. Comparison of calculated 61-broad-group and 174-fine-group fast-neutron spectra with the measured spectrum on centerline $63.2 \mathrm{~cm}$ behind Configuration VI.F 
Table 17. Sensitivities of Neutron Fluxes to Neutron Cross Sections of Various Elements Comprising the Shields of Configuration II.D (1-2)

\begin{tabular}{cccc}
\hline & \multicolumn{3}{c}{ Response } \\
\cline { 3 - 4 } Element & $E>1.1 \mathrm{MeV}$ Flux & \multicolumn{1}{l}{ E.12 MeV Flux } & Total Flux \\
\hline $\mathrm{B}-10$ & -0.859 & -1.285 & -1.623 \\
$\mathrm{~B}-11$ & -2.585 & -3.147 & -3.218 \\
$\mathrm{C}$ & -0.766 & -1.037 & -1.029 \\
$\mathrm{O}$ & -0.543 & -0.602 & -0.631 \\
$\mathrm{Na}$ & -0.158 & -0.158 & -0.161 \\
$\mathrm{Al}$ & -1.478 & -1.434 & -1.433 \\
$\mathrm{~Pb}$ & -0.576 & -0.366 & -0.357 \\
$\mathrm{U}-235$ & 0.238 & 0.263 & 0.264 \\
$\mathrm{U}-238$ & -1.237 & -1.246 & -1.238 \\
$\mathrm{Cr}$ & -0.842 & -0.675 & -0.635 \\
$\mathrm{Ni}$ & -0.436 & -0.351 & -0.341 \\
$\mathrm{Mn}$ & -0.127 & -0.115 & -0.114 \\
$\mathrm{Fe}$ & -4.811 & -4.057 & -3.861 \\
\hline
\end{tabular}

Table 18. Sensitivities of Neutron Fluxes to Neutron Cross Sections of Various Elements Comprising the Shields of Configuration IV.H

\begin{tabular}{cccc}
\hline & \multicolumn{3}{c}{ Response } \\
\cline { 3 - 4 } Element & $E>1.1 \mathrm{MeV}$ Flux & $\mathrm{E}>0.12 \mathrm{MeV}$ Flux & Total Flux \\
\hline B-10 & -0.301 & -0.377 & -0.635 \\
$\mathrm{~B}-11$ & -0.849 & -1.021 & -1.393 \\
$\mathrm{C}$ & -4.891 & -6.551 & -7.780 \\
$\mathrm{O}$ & -0.579 & -0.567 & -0.683 \\
$\mathrm{Na}$ & -0.165 & -0.163 & -0.183 \\
$\mathrm{Al}$ & -2.112 & -2.067 & -2.112 \\
$\mathrm{U}-235$ & 0.213 & 0.247 & 0.299 \\
$\mathrm{U}-238$ & -1.269 & -1.287 & -1.431 \\
$\mathrm{Cr}$ & -1.858 & -1.543 & -1.500 \\
$\mathrm{Ni}$ & -0.974 & -0.823 & -1.092 \\
$\mathrm{Mn}$ & -0.260 & -0.244 & -0.410 \\
$\mathrm{Fe}$ & -8.814 & -7.282 & -7.602 \\
\hline
\end{tabular}




\subsection{CONCLUSIONS}

Measured data from the Radial Shield Altenuation Experiment have been analyzed. Calculations were performed with the DOT-IV two-dimensional discrete ordinates radiation transport code for each of the experimental configurations of the Radial Shield Attenuation Experiment and calculated results were compared with measured results. In general, the calculated results were in good agreement with the measured results. Some cases of disagreement were attributed to a high background contribution to the measured results. However, in other cases, the data appeared to be lacking. Configurations containing large amounts of $\mathrm{B}_{4} \mathrm{C}$ gave low calculated results because of over-attenuation by the ENDF/B versions IV and $V{ }^{11} B$ data set. $A$ LENDL set, which differed from the ENDF set mainly at high energies, gave much improved results but substantially overpredicted portions of the neutron spectra and substantially underpredicted others. It is preferable to the ENDF set, but the need for better high-energy cross-section definition for this set is indicated by the results obtained even with a fine-group structure. The broad-group structure was found to be partially responsible for the underprediction of radiation transmission through graphite. The integrated fast-neutron spectrum obtained using a fine-group structure indicated much better agreement with the measured spectrum for a configuration with much graphite. Detector responses for configurations having $\mathrm{B}_{4} \mathrm{C}$ preceding sodium tended to be overpredicted. Finally, detector responses for Configurations VII (with 30 to $45 \mathrm{~cm}$ stainless steel) were slightly underpredicted. Larger differences than those seen here were noted between fine-group and broad-group results when the 61-group library was tested using 1-D mockups containing slabs of steel and sodium ${ }^{6}$. Thus, this slight underprediction is attributed to the broad-group structure.

The results of this analysis have a few implications for shield design analyses.

(1) Analyses using the ENDF ${ }^{11} \mathrm{~B}$ cross section set will probably underpredict neutron transmission through $\mathrm{B}_{4} \mathrm{C}$ shields. Though not perfect, the LENDL ${ }^{11} \mathrm{~B}$ set is preferable to the ENDF set.

(2) Analyses of $\mathrm{B}_{4} \mathrm{C}$ shields in deep sodium are likely to overpredict neutron transmission through the $\mathrm{B}_{4} \mathrm{C}$ and the sodium that follows.

(3) Fine group calculations may be required for accurate calculation of neutron transmission through thick steel or graphite shields. 


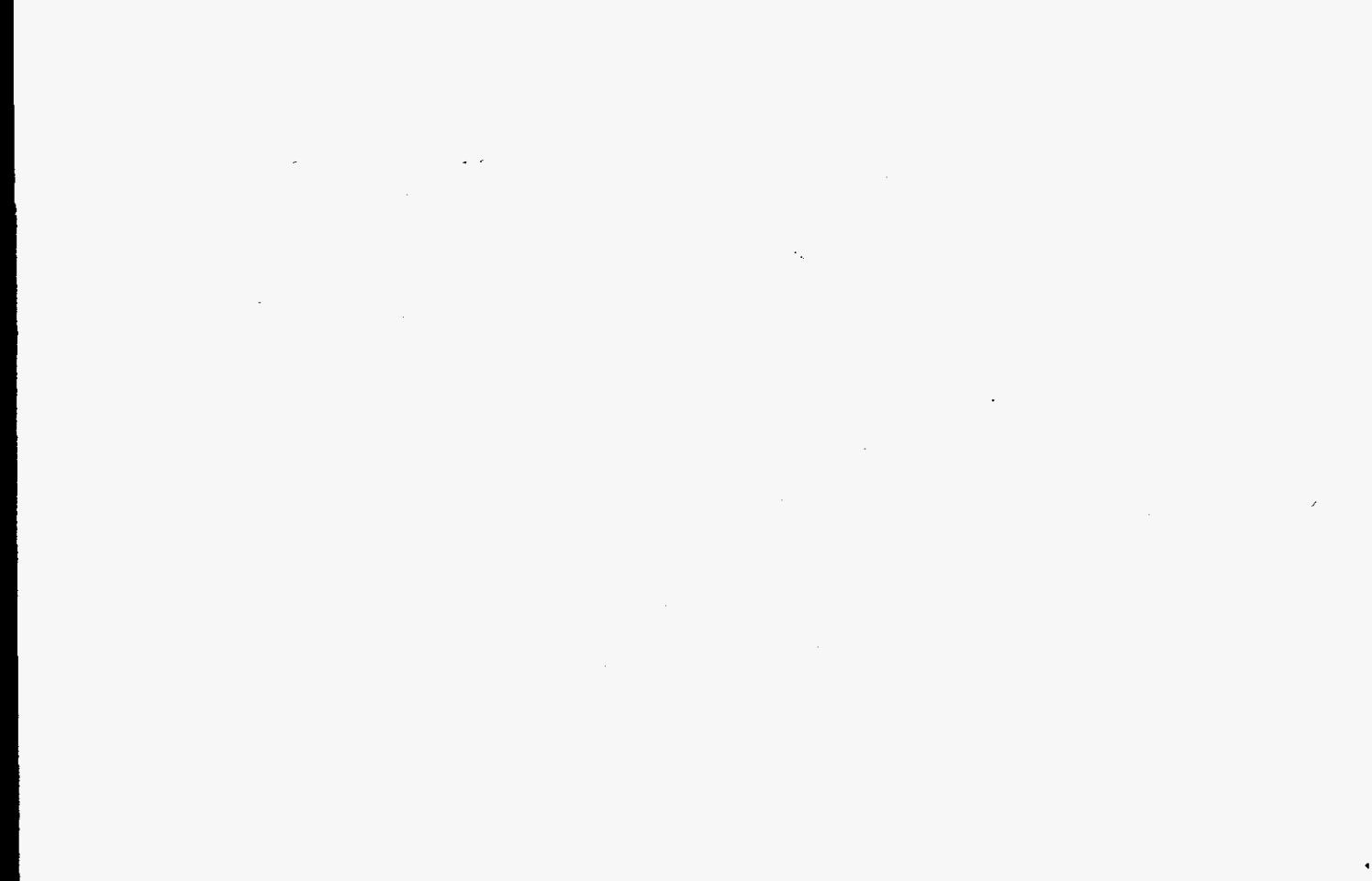


APPENDIX A

EXPERIMENTAL PROGRAM PLAN FOR THE

JASPER RADIAL SHIELD ATTENUATION EXPERIMENT 


\section{Bxperimental Program Plan for the JASPER Radial Shield Attenuation Experiment}

I. Spectrum Modifier (SM) for near core shield designs

A. SMl $(10.27 \mathrm{~cm} \mathrm{Fe}+9.17 \mathrm{~cm} \mathrm{Al}+2.54 \mathrm{~cm}$ Boral + 20.32cm Radial Blanket)

1. NE-213 and hydrogen counter measurements on centerline as close as feasible to SMI.

2. 3-, 5-, 10-in Bonner ball on centerline at same location as NE-213.

3. 5-in BB horizontal traverse at $30 \mathrm{~cm}$ behind configuration.

4. 3-, 5-, 8-, 10-in BB measurements on centerline:
a. at $30 \mathrm{~cm}$ behind configuration
b. at $304.8 \mathrm{~cm}$ from reactor centerline (Foreground and Background)
c. at $457.2 \mathrm{~cm}$ from reactor centerline (Foreground and Background).

5. INS ${ }^{3} \mathrm{He}$ BB measurements on centerline:
a. at $30 \mathrm{~cm}$ behind configuration
b. at $304.8 \mathrm{~cm}$ from reactor centerline (Foreground and Background)
c. at $457.2 \mathrm{~cm}$ from reactor centerline (Foreground and Background).

II. SM + Stainless Steel and $\mathrm{B}_{4} \mathrm{C}$ combinations (near core shield)

A. $\mathrm{SMI}+1.27 \mathrm{~cm} \mathrm{Al}+15.24 \mathrm{~cm} \mathrm{SS}$

1. 5-in $\mathrm{BB}$ horizontal traverse at $30 \mathrm{~cm}$ behind configuration.

2. 3-, 5-, 8-, 10-in BB measurements on centerline:
a. at $30 \mathrm{~cm}$ behind configuration
b. at $304.8 \mathrm{~cm}$ from reactor centerline (Foreground and Background).


B. $\mathrm{SMl}+1.27 \mathrm{~cm} \mathrm{Al}+15.24 \mathrm{~cm} \mathrm{SS}+1.27 \mathrm{~cm} \mathrm{Al}+15.24 \mathrm{~cm} \mathrm{~B} \mathrm{~B}_{4} \mathrm{C}+$ $2.54 \mathrm{~cm}$ SS

1. 3-, 5-, 8-, 10-in BB measurements on centerline:

a. at $30 \mathrm{~cm}$ behind configuration

b. at $304.8 \mathrm{~cm}$ from reactor centerline (Foreground and Background).

C. $\quad \mathrm{SMI}+1.27 \mathrm{~cm} \mathrm{Al}+15.24 \mathrm{~cm} \mathrm{SS}+1.27 \mathrm{~cm} \mathrm{Al}+15.24 \mathrm{~cm} \mathrm{~B} \mathrm{~B}_{4} \mathrm{C}+$ $2.54 \mathrm{~cm} \mathrm{SS}+1.27 \mathrm{~cm} \mathrm{Al}+10.16 \mathrm{~cm} \mathrm{~B} \mathrm{~B}_{4} \mathrm{C}+5.08 \mathrm{~cm} \mathrm{~B} \mathrm{~B}_{4} \mathrm{C}$

1. 3-, 5-, 8-, 10-in BB measurements on centerline:

a. at $30 \mathrm{~cm}$ behind configuration

b. at $304.8 \mathrm{~cm}$ from reactor centerline (Foreground and Background).

D. $\quad \mathrm{SMI}+1.27 \mathrm{~cm} \mathrm{Al}+15.24 \mathrm{~cm} \mathrm{SS}+1.27 \mathrm{~cm} \mathrm{Al}+15.24 \mathrm{~cm} \mathrm{~B} \mathrm{~B}_{4} \mathrm{C}+$ $2.54 \mathrm{~cm} \mathrm{SS}+1.27 \mathrm{~cm} \mathrm{Al}+10.16 \mathrm{~cm} \mathrm{~B} \mathrm{~B}_{4} \mathrm{C}+5.08 \mathrm{~cm} \mathrm{~B} \mathrm{~B}_{4} \mathrm{C}+1.27 \mathrm{~cm} \mathrm{Al}+$ $10.16 \mathrm{~cm} \mathrm{~B} \mathrm{~B}_{4} \mathrm{C}+5.08 \mathrm{~cm} \mathrm{~B} \mathrm{~B}_{4} \mathrm{C}$

1. NE-213 and hydrogen counter measurements on centerline as close as feasible behind configuration.

2. 3-, 5-, 10-in BB measurements on centerline at same location as $\mathrm{NE}-213$.

3. 5-in BB horizontal traverse at $30 \mathrm{~cm}$ behind configuration.

4. 3-, 5-, 8-, 10-in BB measurements on centerline:

a. at $30 \mathrm{~cm}$ behind configuration

b. at $304.8 \mathrm{~cm}$ from reactor centerline (Foreground and Background) .

5. INS ${ }^{3} \mathrm{He} \mathrm{BB}$ measurements on centerline:

a. at $30 \mathrm{~cm}$ behind configuration

b. at $304.8 \mathrm{~cm}$ from reactor centerline (Foreground and Background).

E. $\quad \mathrm{SMI}+1.27 \mathrm{~cm} \mathrm{Al}+15.24 \mathrm{~cm} \mathrm{SS}+1.27 \mathrm{~cm} \mathrm{Al}+15.24 \mathrm{~cm} \mathrm{~B} \mathrm{~B}_{4} \mathrm{C}+$

$2.54 \mathrm{~cm} \mathrm{SS}+1.27 \mathrm{~cm} \mathrm{Al}+10.16 \mathrm{~cm} \mathrm{~B} \mathrm{~B}_{4} \mathrm{C}+5.08 \mathrm{~cm} \mathrm{~B} \mathrm{~B}_{4} \mathrm{C}+1.27 \mathrm{~cm} \mathrm{Al}+$ $10.16 \mathrm{~cm} \mathrm{~B} \mathrm{~B}_{4} \mathrm{C}+5.08 \mathrm{~cm} \mathrm{~B} \mathrm{~B}_{4} \mathrm{C}+30.48 \mathrm{~cm} \mathrm{Na}$

1. 5-in BB horizontal traverse at $30 \mathrm{~cm}$ behind configuration. 
2. Bare, Cd-covered, 3-, 5-, 8-, 10-in BB measurements on centerline:
a. at $30 \mathrm{~cm}$ behind configuration
b. at $304.8 \mathrm{~cm}$ from reactor centerline (Foreground and Background).

3. Bare detector on centerline at $30 \mathrm{~cm}$ behind configuration inside partial cadmium enclosure that surrounded the detector except on the side of the last slab in the configuration.

4. Bare detector on centerline at $501.3 \mathrm{~cm}$ point with cadmium sheet over face of last slab in the configuration.

III. $\mathrm{SM}+\mathrm{SS}$ and $\mathrm{B}_{4} \mathrm{C}$

A. SMI $+10.16 \mathrm{~cm} \mathrm{SS}$

1. 5-in BB horizontal traverse at $30 \mathrm{~cm}$ behind configuration.

2. 3-, 5-, 8-, 10-in $\mathrm{BB}$ measurements on centerline:

a. at $30 \mathrm{~cm}$ behind configuration

b. at $304.8 \mathrm{~cm}$ from reactor centerline (Foreground and Background).

B. $\mathrm{SMI}+10.16 \mathrm{~cm} \mathrm{SS}+5.08 \mathrm{~cm} \mathrm{~B} \mathrm{~B}_{4} \mathrm{C}$

1. 5-in BB horizontal traverse at $30 \mathrm{~cm}$ behind configuration.

2. 3-, 5-, 8-, 10-in BB measurements on centerline:

a. at $30 \mathrm{~cm}$ behind configuration

b. at $304.8 \mathrm{~cm}$ from reactor centerline (Foreground and Background) .

C. $\quad \mathrm{SMI}+20.32 \mathrm{~cm} \mathrm{SS}$

1. 5-in $\mathrm{BB}$ horizontal traverse at $30 \mathrm{~cm}$ behind configuration.

2. 3-, 5-, 8-, 10-in BB measurements on centerline:

a. at $30 \mathrm{~cm}$ behind configuration

b. at $304.8 \mathrm{~cm}$ from reactor centerline (Foreground and Background) . 
D. $\quad \mathrm{SMI}+20.32 \mathrm{~cm} \mathrm{SS}+5.08 \mathrm{~cm} \mathrm{~B} \mathrm{~B}_{4} \mathrm{C}$

1. 3-, 5-, 8-, 10-in BB measurements on centerline:

a. at $30 \mathrm{~cm}$ behind configuration

b. at $304.8 \mathrm{~cm}$ from reactor centerline (Foreground and Background).

E. $\quad \mathrm{SMZ}+20.32 \mathrm{~cm} \mathrm{SS}+5.08 \mathrm{~cm} \mathrm{~B}_{4} \mathrm{C}+5.08 \mathrm{~cm} \mathrm{~B} \mathrm{~B}_{4} \mathrm{C}$

1. 5-in $\mathrm{BB}$ horizontal traverse at $30 \mathrm{~cm}$ behind configuration.

2. 3-, 5-, 8-, 10-in BB measurements on centerline:

a. at $30 \mathrm{~cm}$ behind configuration

b. at $304.8 \mathrm{~cm}$ from reactor centerline (Foreground and Background).

IV. $S M+$ Graphite and $B_{4} C$ (tank type shield reference)

A. $\quad \mathrm{SMI}+1.27 \mathrm{~cm} \mathrm{Al}+15.24 \mathrm{~cm} \mathrm{SS}+1.27 \mathrm{~cm} \mathrm{Al}+10.16 \mathrm{~cm} \mathrm{C}+5.08 \mathrm{~cm}$ SS

1. 5-in $\mathrm{BB}$ horizontal traverse at $30 \mathrm{~cm}$ behind configuration.

2. 3-, 5-, 8-, 10-in BB measurements on centerline:

a. at $30 \mathrm{~cm}$ behind configuration

b. at $304.8 \mathrm{~cm}$ from reactor centerline (Foreground and Background).

B. $\quad \mathrm{SMI}+1.27 \mathrm{~cm} \mathrm{Al}+15.24 \mathrm{~cm} \mathrm{SS}+1.27 \mathrm{~cm} \mathrm{Al}+10.16 \mathrm{~cm} \mathrm{C}+5.08 \mathrm{~cm}$ $\mathrm{SS}+1.27 \mathrm{~cm} \mathrm{Al}+10.16 \mathrm{~cm} \mathrm{C}+5.08 \mathrm{~cm} \mathrm{SS}$

1. 3-, 5-, 8-, 10-in BB measurements on centerline:

a. at $30 \mathrm{~cm}$ behind configuration

b. at $304.8 \mathrm{~cm}$ from reactor centerline (Foreground and Background).

C. $\mathrm{SMl}+1.27 \mathrm{~cm} \mathrm{Al}+15.24 \mathrm{~cm} \mathrm{SS}+1.27 \mathrm{~cm} \mathrm{Al}+10.16 \mathrm{~cm} \mathrm{C}+5.08 \mathrm{~cm}$ $\mathrm{SS}+1.27 \mathrm{~cm} \mathrm{AI}+10.16 \mathrm{~cm} \mathrm{C}+5.08 \mathrm{~cm} \mathrm{SS}+1.27 \mathrm{~cm} \mathrm{AI}+15.24 \mathrm{~cm}$ $\mathrm{B}_{4} \mathrm{C}+2.54 \mathrm{~cm} \mathrm{SS}$

1. Ne-213 and hydrogen counter measurements on centerline as close as feasible behind SS. 
2. 3-, 5-, 10-in BB measurements on centerline at same location as NE-213.

3. 5-in BB horizontal traverse at $30 \mathrm{~cm}$ behind configuration.

4. 3-, 5-, 8-, 10-in BB measurements on centerline:
a. at $30 \mathrm{~cm}$ behind configuration
b. at $304.8 \mathrm{~cm}$ from reactor centerline (Foreground and Background)
c. at $457.2 \mathrm{~cm}$ from reactor centerline (Foreground and Background) .

5. INS ${ }^{3} \mathrm{He} \mathrm{BB}$ measurements on centerline:
a. at $30 \mathrm{~cm}$ behind configuration
b. at $304.8 \mathrm{~cm}$ from reactor centerline (Foreground and Background)
c. at $457.2 \mathrm{~cm}$ from reactor centerline (Foreground and Background).

D. $\quad \mathrm{SMI}+1.27 \mathrm{~cm} \mathrm{Al}+15.24 \mathrm{~cm} \mathrm{SS}+1.27 \mathrm{~cm} \mathrm{Al}+10.16 \mathrm{~cm} \mathrm{C}+5.08 \mathrm{~cm}$ $\mathrm{SS}+1.27 \mathrm{~cm} \mathrm{Al}+10.16 \mathrm{~cm} \mathrm{C}+5.08 \mathrm{~cm} \mathrm{SS}+1.27 \mathrm{~cm} \mathrm{Al}+15.24 \mathrm{~cm}$ $\mathrm{B}_{4} \mathrm{C}+2.54 \mathrm{~cm} \mathrm{SS}+2.54 \mathrm{~cm} \mathrm{Al}+5.08 \mathrm{~cm} \mathrm{SS}$

1. 3-, 5-, 8-, 10-in BB measurements on centerline:
a. at $30 \mathrm{~cm}$ behind configuration
b. at $457.2 \mathrm{~cm}$ from reactor centerline (Foreground and Background).

E. $\quad \mathrm{SMI}+1.27 \mathrm{~cm} \mathrm{Al}+15.24 \mathrm{~cm} \mathrm{SS}+1.27 \mathrm{~cm} \mathrm{Al}+10.16 \mathrm{~cm} \mathrm{C}+5.08 \mathrm{~cm}$ $\mathrm{SS}+1.27 \mathrm{~cm} \mathrm{Al}+10.16 \mathrm{~cm} \mathrm{C}+5.08 \mathrm{~cm} \mathrm{SS}+1.27 \mathrm{~cm} \mathrm{Al}+15.24 \mathrm{~cm}$ $\mathrm{B}_{4} \mathrm{C}+2.54 \mathrm{~cm} \mathrm{SS}+2.54 \mathrm{~cm} \mathrm{Al}+5.08 \mathrm{~cm} \mathrm{SS}+1.27 \mathrm{~cm} \mathrm{Al}+$ $10.16 \mathrm{~cm} \mathrm{C}+5.08 \mathrm{~cm}$ SS

1. 3-, 5-, 8-, 10-in BB measurements on centerline:
a. at $30 \mathrm{~cm}$ behind configuration
b. at $457.2 \mathrm{~cm}$ from reactor centerline (Foreground and Background).

F. $\quad \mathrm{SMI}+1.27 \mathrm{~cm} \mathrm{Al}+15.24 \mathrm{~cm} \mathrm{SS}+1.27 \mathrm{~cm} \mathrm{Al}+10.16 \mathrm{~cm} \mathrm{C}+5.08 \mathrm{~cm}$ $\mathrm{SS}+1.27 \mathrm{~cm} \mathrm{Al}+10.16 \mathrm{~cm} \mathrm{C}+5.08 \mathrm{~cm} \mathrm{SS}+1.27 \mathrm{~cm} \mathrm{Al}+15.24 \mathrm{~cm}$ $\mathrm{B}_{4} \mathrm{C}+2.54 \mathrm{~cm} \mathrm{SS}+2.54 \mathrm{~cm} \mathrm{Al}+5.08 \mathrm{~cm} \mathrm{SS}+1.27 \mathrm{~cm} \mathrm{Al}+$ $10.16 \mathrm{~cm} \mathrm{C}+5.08 \mathrm{~cm} \mathrm{SS}+1.27 \mathrm{~cm} \mathrm{Al}+10.16 \mathrm{~cm} \mathrm{C}+5.08 \mathrm{~cm} \mathrm{SS}$ 
1. 3-, 5-, 8-, 10-in BB measurements on centerline:

a. at $30 \mathrm{~cm}$ behind configuration

b. at $457.2 \mathrm{~cm}$ from reactor centerline (Foreground and Background).

2. 3-, 5-, 8-, 10-in BB measurements on centerline as close as feasible:

a. Foreground (same location as background)

b. Background $(30.48 \mathrm{~cm} \mathrm{LiH}$ between detector and configuration).

G. $\mathrm{SMl}+1.27 \mathrm{~cm} \mathrm{Al}+15.24 \mathrm{~cm} \mathrm{SS}+1.27 \mathrm{~cm} \mathrm{Al}+10.16 \mathrm{~cm} \mathrm{C}+5.08 \mathrm{~cm}$ $\mathrm{SS}+1.27 \mathrm{~cm} \mathrm{Al}+10.16 \mathrm{~cm} \mathrm{C}+5.08 \mathrm{~cm} \mathrm{SS}+1.27 \mathrm{~cm} \mathrm{Al}+15.24 \mathrm{~cm}$ $\mathrm{B}_{4} \mathrm{C}+2.54 \mathrm{~cm} \mathrm{SS}+2.54 \mathrm{~cm} \mathrm{Al}+5.08 \mathrm{~cm} \mathrm{SS}+1.27 \mathrm{~cm} \mathrm{Al}+10.16 \mathrm{~cm}$ $\mathrm{C}+5.08 \mathrm{~cm} \mathrm{SS}+1.27 \mathrm{~cm} \mathrm{Al}+10.16 \mathrm{~cm} \mathrm{C}+5.08 \mathrm{~cm} \mathrm{SS}+1.27 \mathrm{~cm} \mathrm{Al}$ $+10.16 \mathrm{~cm} \mathrm{C}+5.08 \mathrm{~cm}$ SS

1. 3-, 5-, 8-, 10-in BB measurements on centerline as close as feasible:
a. Foreground (same location as for background)
b. Background $(30.48 \mathrm{~cm} \mathrm{LiH}$ between detector and configuration).

2. INS ${ }^{3} \mathrm{He} \mathrm{BB}$ measurements on centerline as close as feasible:
a. Foreground (same location as for background)
b. Background (30.48cm IiH between detector and configuration).

H. $\quad \mathrm{SMI}+1.27 \mathrm{~cm} \mathrm{Al}+15.24 \mathrm{~cm} \mathrm{SS}+1.27 \mathrm{~cm} \mathrm{Al}+10.16 \mathrm{~cm} \mathrm{C}+5.08 \mathrm{~cm}$ $\mathrm{SS}+1.27 \mathrm{~cm} \mathrm{Al}+10.16 \mathrm{~cm} \mathrm{C}+5.08 \mathrm{~cm} \mathrm{SS}+1.27 \mathrm{~cm} \mathrm{Al}+15.24 \mathrm{~cm}$ $\mathrm{B}_{4} \mathrm{C}+2.54 \mathrm{~cm} \mathrm{SS}+2.54 \mathrm{~cm} \mathrm{Al}+5.08 \mathrm{~cm} \mathrm{SS}+1.27 \mathrm{~cm} \mathrm{Al}+10.16 \mathrm{~cm}$ $\mathrm{C}+5.08 \mathrm{~cm} \mathrm{SS}+1.27 \mathrm{~cm} \mathrm{Al}+10.16 \mathrm{~cm} \mathrm{C}+5.08 \mathrm{~cm} \mathrm{SS}+1.27 \mathrm{~cm} \mathrm{Al}$ $+10.16 \mathrm{~cm} \mathrm{C}+5.08 \mathrm{~cm} \mathrm{SS}+5.08 \mathrm{~cm} \mathrm{SS}$

1. 3-, 5-, 8-, 10-in $\mathrm{BB}$ measurements on centerline as close as feasible:
a. Foreground (same location as for background)
b. Background $(30.48 \mathrm{~cm} \mathrm{LiH}$ between detector and configuration).


I. $\quad \mathrm{SMI}+1.27 \mathrm{~cm} \mathrm{Al}+15.24 \mathrm{~cm} \mathrm{SS}+1.27 \mathrm{~cm} \mathrm{Al}+10.16 \mathrm{~cm} \mathrm{C}+5.08 \mathrm{~cm}$ $\mathrm{SS}+1.27 \mathrm{~cm} \mathrm{Al}+10.16 \mathrm{~cm} \mathrm{C}+5.08 \mathrm{~cm} \mathrm{SS}+1.27 \mathrm{~cm} \mathrm{Al}+15.24 \mathrm{~cm}$ $\mathrm{B}_{4} \mathrm{C}+2.54 \mathrm{~cm} \mathrm{SS}+2.54 \mathrm{~cm} \mathrm{Al}+5.08 \mathrm{~cm} \mathrm{SS}+1.27 \mathrm{~cm} \mathrm{Al}+10.16 \mathrm{~cm}$ $\mathrm{C}+5.08 \mathrm{~cm} \mathrm{SS}+1.27 \mathrm{~cm} \mathrm{Al}+10.16 \mathrm{~cm} \mathrm{C}+5.08 \mathrm{~cm} \mathrm{SS}+1.27 \mathrm{~cm} \mathrm{Al}$ $+10.16 \mathrm{~cm} \mathrm{C}+5.08 \mathrm{~cm} \mathrm{SS}+5.08 \mathrm{~cm} \mathrm{SS}+30.48 \mathrm{~cm} \mathrm{Na}$

1. bare, od-covered, 3-, 5-, 8-, 10-in BB measurements on centerline as close as feasible:
a. Foreground (same as for background)
b. Background $(30.48 \mathrm{~cm} \mathrm{IiH}$ between detector and configuration).

IA. $\mathrm{SMI}+1.27 \mathrm{~cm} \mathrm{Al}+15.24 \mathrm{~cm} \mathrm{SS}+1.27 \mathrm{~cm} \mathrm{Al}+10.16 \mathrm{~cm} \mathrm{C}+5.08 \mathrm{~cm}$ $\mathrm{SS}+1.27 \mathrm{~cm} \mathrm{Al}+10.16 \mathrm{~cm} \mathrm{C}+5.08 \mathrm{~cm} \mathrm{SS}+1.27 \mathrm{~cm} \mathrm{Al}+5.24 \mathrm{~cm}$ $\mathrm{B}_{4} \mathrm{C}+2.54 \mathrm{~cm} \mathrm{SS}+2.54 \mathrm{~cm} \mathrm{Al}+5.08 \mathrm{~cm} \mathrm{SS}+1.27 \mathrm{~cm} \mathrm{Al}+10.16 \mathrm{~cm}$ $\mathrm{C}+5.08 \mathrm{~cm} \mathrm{SS}+1.27 \mathrm{~cm} \mathrm{Al}+10.16 \mathrm{~cm} \mathrm{C}+5.08 \mathrm{~cm} \mathrm{SS}+1.27 \mathrm{~cm} \mathrm{Al}$ $+10.16 \mathrm{~cm} \mathrm{C}+5.08 \mathrm{~cm} \mathrm{SS}+5.08 \mathrm{~cm} \mathrm{SS}+30.48 \mathrm{~cm} \mathrm{Na}+30 \mathrm{~cm}$ Void + $30.48 \mathrm{~cm} \mathrm{Na}$

1. bare, co-covered, 3-, 5-, 8-, 10-in BB on centerline in $30 \mathrm{~cm}$ void.

J. $\mathrm{SMI}+1.27 \mathrm{~cm} \mathrm{Al}+15.24 \mathrm{~cm} \mathrm{SS}+1.27 \mathrm{~cm} \mathrm{Al}+10.16 \mathrm{~cm} \mathrm{C}+5.08 \mathrm{~cm} \mathrm{SS}+$ $1.27 \mathrm{~cm} \mathrm{Al}+10.16 \mathrm{~cm} \mathrm{C}+5.08 \mathrm{~cm} \mathrm{SS}+1.27 \mathrm{~cm} \mathrm{Al}+15.24 \mathrm{~cm} \mathrm{~B} \mathrm{~B}_{4} \mathrm{C}+$ $2.54 \mathrm{~cm} \mathrm{SS}+2.54 \mathrm{~cm} \mathrm{Al}+5.08 \mathrm{~cm} \mathrm{SS}+1.27 \mathrm{~cm} \mathrm{Al}+10.16 \mathrm{~cm} \mathrm{C}+5.08 \mathrm{~cm}$ $\mathrm{SS}+1.27 \mathrm{~cm} \mathrm{Al}+10.16 \mathrm{~cm} \mathrm{C}+5.08 \mathrm{~cm} \mathrm{SS}+1.27 \mathrm{~cm} \mathrm{Al}+10.16 \mathrm{~cm} \mathrm{C} \mathrm{+}$ $5.08 \mathrm{~cm} \mathrm{SS}+5.08 \mathrm{~cm} \mathrm{SS}+61 \mathrm{~cm} \mathrm{Na}+30 \mathrm{~cm} \mathrm{Void}+30.48 \mathrm{~cm} \mathrm{Na}$

1. bare, cd-covered, 3-, 5-, 8-, 10-in BB on centerline in $30 \mathrm{~cm}$ void.

2. INS ${ }^{3} \mathrm{He} \mathrm{BB}$ on centerline in $30 \mathrm{~cm}$ void.

K. $\mathrm{SMI}+1.27 \mathrm{~cm} \mathrm{Al}+15.24 \mathrm{~cm} \mathrm{SS}+1.27 \mathrm{~cm} \mathrm{Al}+10.16 \mathrm{~cm} \mathrm{C}+5.08 \mathrm{~cm} \mathrm{SS}+$ $1.27 \mathrm{~cm} \mathrm{Al}+10.16 \mathrm{~cm} \mathrm{C}+5.08 \mathrm{~cm} \mathrm{SS}+1.27 \mathrm{~cm} \mathrm{Al}+15.24 \mathrm{~cm} \mathrm{~B} \mathrm{~B}_{4} \mathrm{C}+$ $2.54 \mathrm{~cm} \mathrm{SS}+2.54 \mathrm{~cm} \mathrm{Al}+5.08 \mathrm{~cm} \mathrm{SS}+1.27 \mathrm{~cm} \mathrm{Al}+10.16 \mathrm{~cm} \mathrm{C}+5.08 \mathrm{~cm}$ $\mathrm{SS}+1.27 \mathrm{~cm} \mathrm{Al}+10.16 \mathrm{~cm} \mathrm{C}+5.08 \mathrm{~cm} \mathrm{SS}+1.27 \mathrm{~cm} \mathrm{Al}+10.16 \mathrm{~cm} \mathrm{C}+$ $5.08 \mathrm{~cm} \mathrm{SS}+5.08 \mathrm{~cm} \mathrm{SS}+91 \mathrm{~cm} \mathrm{Na}+30 \mathrm{~cm} \mathrm{Void}+30.48 \mathrm{~cm} \mathrm{Na}$

1. bare, cd-covered, 3-, 5-, 8-, 10-in BB on centerline in $30 \mathrm{~cm}$ void.

L. $\quad \mathrm{SMI}+1.27 \mathrm{~cm} \mathrm{Al}+15.24 \mathrm{~cm} \mathrm{SS}+1.27 \mathrm{~cm} \mathrm{Al}+10.16 \mathrm{~cm} \mathrm{C}+5.08 \mathrm{~cm} \mathrm{SS}+$ $1.27 \mathrm{~cm} \mathrm{Al}+10.16 \mathrm{~cm} \mathrm{C}+5.08 \mathrm{~cm} \mathrm{SS}+1.27 \mathrm{~cm} \mathrm{Al}+15.24 \mathrm{~cm} \mathrm{~B} \mathrm{~B}_{4}+$ $2.54 \mathrm{~cm} \mathrm{SS}+2.54 \mathrm{~cm} \mathrm{Al}+5.08 \mathrm{~cm} \mathrm{SS}+1.27 \mathrm{~cm} \mathrm{Al}+10.16 \mathrm{~cm} \mathrm{C}+5.08 \mathrm{~cm}$ $\mathrm{SS}+1.27 \mathrm{~cm} \mathrm{Al}+10.16 \mathrm{~cm} \mathrm{C}+5.08 \mathrm{~cm} \mathrm{SS}+1.27 \mathrm{~cm} \mathrm{Al}+10.16 \mathrm{~cm} \mathrm{C}+$ $5.08 \mathrm{~cm} \mathrm{SS}+5.08 \mathrm{~cm} \mathrm{SS}+122 \mathrm{~cm} \mathrm{Na}+30 \mathrm{~cm} \mathrm{Void}+30.48 \mathrm{~cm} \mathrm{Na}$

1. bare, od-covered, 3-, 5-, 8-, 10-in BB on centerline in $30 \mathrm{~cm}$ void. 
M. $\quad \mathrm{SMI}+1.27 \mathrm{~cm} \mathrm{Al}+15.24 \mathrm{~cm} \mathrm{SS}+1.27 \mathrm{~cm} \mathrm{Al}+10.16 \mathrm{~cm} \mathrm{C}+5.08 \mathrm{~cm} \mathrm{SS}+$ $1.27 \mathrm{~cm} \mathrm{Al}+10.16 \mathrm{~cm} \mathrm{C}+5.08 \mathrm{~cm} \mathrm{SS}+1.27 \mathrm{~cm} \mathrm{Al}+15.24 \mathrm{~cm} \mathrm{~B} \mathrm{~B}_{4} \mathrm{C}+$

$2.54 \mathrm{~cm} \mathrm{SS}+2.54 \mathrm{~cm} \mathrm{Al}+5.08 \mathrm{~cm} \mathrm{SS}+1.27 \mathrm{~cm} \mathrm{Al}+10.16 \mathrm{~cm} \mathrm{C}+5.08 \mathrm{~cm}$ $\mathrm{SS}+1.27 \mathrm{~cm} \mathrm{Al}+10.16 \mathrm{~cm} \mathrm{C}+5.08 \mathrm{~cm} \mathrm{SS}+1.27 \mathrm{~cm} \mathrm{Al}+10.16 \mathrm{~cm} \mathrm{C}+$

$5.08 \mathrm{~cm} \mathrm{SS}+5.08 \mathrm{~cm} \mathrm{SS}+152.4 \mathrm{~cm} \mathrm{Na}+30 \mathrm{~cm}$ Void $+30.48 \mathrm{~cm} \mathrm{Na}$

1. bare, cd-covered, 3-, 5-, 8-, 10-in BB on centerline in 30cm void.

2. INS ${ }^{3} \mathrm{He} \mathrm{BB}$ on centerline in $30 \mathrm{~cm}$ void.

V. $S M+S S+B_{4} C($ IHX Shield $)$

A. SML $(10.27 \mathrm{~cm} \mathrm{Fe}+9.17 \mathrm{~cm} \mathrm{Al}+2.54 \mathrm{~cm} \mathrm{Boral}+182.88 \mathrm{~cm} \mathrm{Na})$

1. 5 -in $\mathrm{BB}$ horizontal traverse through midplane at $30 \mathrm{~cm}$ behind configuration.

2. Bare, cd-covered, 3-, 5-, 8-, 10-in BB measurements on centerline:

a. at $30 \mathrm{~cm}$ behind configuration

b. at $501.3 \mathrm{~cm}$ from reactor centerline (Foreground and Background).

3. Bare detector on centerline at $30 \mathrm{~cm}$ behind configuration inside partial cadmium enclosure that surrounded the detector except on the side of the last slab in the configuration.

4. Bare detector on centerline at $501.3 \mathrm{~cm}$ point with cadmium sheet over face of last slab in the configuration.

5. INS ${ }^{3} \mathrm{He} \mathrm{BB}$ measurements on centerline:

a. at $30 \mathrm{~cm}$ behind configuration

b. at $501.3 \mathrm{~cm}$ from reactor centerline (Foreground and Background).

B. $\quad S M 2+5.08 \mathrm{~cm}$ SS

1. 5-in BB horizontal traverse through midplane at $30 \mathrm{~cm}$ behind configuration.

2. Bare, cd-covered, 3-, 5-, 8-, 10-in BB measurements on centerline:

a. at $30 \mathrm{~cm}$ behind configuration 

b. at $501.3 \mathrm{~cm}$ from reactor centerline (Foreground and Background).

3. Bare detector on centerline at $30 \mathrm{~cm}$ behind configuration inside partial cadmium enclosure that surrounded the detector except on the side of the last slab in the configuration.

4. Bare detector on centerline at $501.3 \mathrm{~cm}$ point with cacmium sheet over face of last slab in the configuration.

c. $\mathrm{SM} 2+5.08 \mathrm{~cm} \mathrm{SS}+30.48 \mathrm{~cm} \mathrm{Na}$

1. Bare, od-covered, 3-, 5-, 8-, 10-in BB measurements on centerline:
a. at $30 \mathrm{~cm}$ behind configuration
b. at $501.3 \mathrm{~cm}$ from reactor centerline (Foreground and Background).

2. Bare detector on centerline at $30 \mathrm{~cm}$ behind configuration inside partial cadmium enclosure that surrounded the detector except on the side of the last slab in the configuration.

3. Bare detector on centerline at $501.3 \mathrm{~cm}$ point with cadmium sheet over face of last slab in the configuration.

D. $\mathrm{SM} 2+5.08 \mathrm{~cm} \mathrm{SS}+61 \mathrm{~cm} \mathrm{Na}$

1. 5-in BB horizontal traverse through midplane at $30 \mathrm{~cm}$ behind configuration.

2. Bare, od-covered, 3-, 5-, 8-, 10-in BB measurements on centerline:
a. at $30 \mathrm{~cm}$ behind configuration
b. at $501.3 \mathrm{~cm}$ from reactor centerline (Foreground and Background).

3. Bare detector on centerline at $30 \mathrm{~cm}$ behind configuration inside partial cadmium enclosure that surrounded the detector except on the side of the last slab in the configuration.

4. Bare detector on centerline at $501.3 \mathrm{~cm}$ point with cadmium sheet over face of last slab in the configuration.

5. INS ${ }^{3} \mathrm{He} \mathrm{BB}$ measurements on centerline:

a. at $30 \mathrm{~cm}$ behind configuration 

b. at $501.3 \mathrm{~cm}$ from reactor centerline (Foreground and
Background).

E. $\quad \mathrm{SM} 2+5.08 \mathrm{~cm} \mathrm{~B} \mathrm{~B}_{4} \mathrm{C}+5.08 \mathrm{~cm} \mathrm{SS}$

1. Bare, od-covered, 3-, 5-, 8-, 10-in BB measurements on centerline:
a. at $30 \mathrm{~cm}$ behind configuration
b. at $501.3 \mathrm{~cm}$ from reactor centerline (Foreground and Background).

2. Bare detector on centerline at $30 \mathrm{~cm}$ behind configuration inside partial cadmium enclosure that surrounded the detector except on the side of the last slab in the configuration.

3. Bare detector on centerline at $501.3 \mathrm{~cm}$ point with cadmium sheet over face of last slab in the configuration.

F. $\quad \mathrm{SN2}+5.08 \mathrm{~cm} \mathrm{~B} \mathrm{~B}_{4} \mathrm{C}+5.08 \mathrm{~cm} \mathrm{SS}+30.48 \mathrm{~cm} \mathrm{Na}$

1. Bare, cd-covered, 3-, 5-, 8-, 10-in BB measurements on centerline:
a. at $30 \mathrm{~cm}$ behind configuration
b. at $501.3 \mathrm{~cm}$ from reactor centerline (Foreground and Background).

2. Bare detector on centerline at $30 \mathrm{~cm}$ behind configuration inside partial cadmium enclosure that surrounded the detector except on the side of the last slab in the configuration.

3. Bare detector on centerline at $501.3 \mathrm{~cm}$ point with cadmium sheet over face of last slab in the configuration.

G. $\quad \mathrm{SM} 2+5.08 \mathrm{~cm} \mathrm{~B} \mathrm{~B}_{4} \mathrm{C}+5.08 \mathrm{~cm} \mathrm{SS}+61 \mathrm{~cm} \mathrm{Na}$

1. 5-in BB horizontal traverse through midplane at $30 \mathrm{~cm}$ behind configuration.

2. Bare, cd-covered, 3-, 5-, 8-, 10-in BB measurements on centerline:
a. at $30 \mathrm{~cm}$ behind configuration
b. at $501.3 \mathrm{~cm}$ from reactor centerline (Foreground and Background).


3. Bare detector on centerline at $30 \mathrm{~cm}$ behind configuration inside partial cadmium enclosure that surrounded the detector except on the side of the last slab in the configuration.

4. Bare detector on centerline at $501.3 \mathrm{~cm}$ point with cadmium sheet over face of last slab in the configuration.

5. INS ${ }^{3} \mathrm{He} \mathrm{BB}$ measurements on centerline:
a. at $30 \mathrm{~cm}$ behind configuration
b. at $501.3 \mathrm{~cm}$ from reactor centerline (Foreground and Background).

H. $\quad S M 2+10.16 \mathrm{~cm} \mathrm{~B} B_{4} C+5.08 \mathrm{~cm} \mathrm{SS}$

1. Bare, cd-covered, 3-, 5-, 8-, 10-in BB measurements on centerline:
a. at $30 \mathrm{~cm}$ behind configuration
b. at $501.3 \mathrm{~cm}$ from reactor centerline (Foreground and Background).

2. Bare detector on centerline at $30 \mathrm{~cm}$ behind configuration inside partial cadnium enclosure that surrounded the detector except on the side of the last slab in the configuration.

3. Bare detector on centerline at $501.3 \mathrm{~cm}$ point with cadmium sheet over face of last slab in the configuration.

I. $\quad \mathrm{SN2}+10.16 \mathrm{~cm} \mathrm{~B} \mathrm{~B}_{4} \mathrm{C}+5.08 \mathrm{~cm} \mathrm{SS}+30.48 \mathrm{~cm} \mathrm{Na}$

1. Bare, cd-covered, 3-, 5-, 8-, 10-in BB measurements on centerline:
a. at $30 \mathrm{~cm}$ behind configuration
b. at $501.3 \mathrm{~cm}$ from reactor centerline (Foreground and Background).

2. Bare detector on centerline at $30 \mathrm{~cm}$ behind configuration inside partial cadmium enclosure that surroumded the detector except on the side of the last slab in the configuration.

3. Bare detector on centerline at $501.3 \mathrm{~cm}$ point with cadmium sheet over face of last slab in the configuration.

J. $\mathrm{SM} 2+10.16 \mathrm{~cm} \mathrm{~B} \mathrm{~B}_{4} \mathrm{C}+5.08 \mathrm{~cm} \mathrm{SS}+61 \mathrm{~cm} \mathrm{Na}$

1. 5-in BB horizontal traverse through midplane at $30 \mathrm{~cm}$ behind configuration. 
2. Bare, cd-covered, 3-, 5-, 8-, 10-in BB measurements on centerline:
a. at $30 \mathrm{~cm}$ behind configuration
b. at $501.3 \mathrm{~cm}$ from reactor centerline (Foreground and Background).

3. Bare detector on centerline at $30 \mathrm{~cm}$ behind configuration inside partial cadmium enclosure that surrounded the detector except on the side of the last slab in the configuration.

4. Bare detector on centerline at $501.3 \mathrm{~cm}$ point with cadmium sheet over face of last slab in the configuration.

5. INS ${ }^{3} \mathrm{He} \mathrm{BB}$ measurements on centerline:
a. at $30 \mathrm{~cm}$ behind configuration
b. at $501.3 \mathrm{~cm}$ from reactor centerline (Foreground and Background).

VI. SM + SS and Graphite (Graphite benchmark)

A. SMl $(10.27 \mathrm{~cm} \mathrm{Fe}+9.17 \mathrm{~cm} \mathrm{Al}+2.54 \mathrm{~cm}$ boral $+20.32 \mathrm{~cm}$ radial blanket) $+1.27 \mathrm{~cm} \mathrm{Al}+15.24 \mathrm{~cm} \mathrm{SS}+10.16 \mathrm{~cm}$ graphite

1. NE-213 and hydrogen counter measurements on centerline as close as feasible behind ss.

2. 3-, 5-, 10-in BB measurements on centerline at same location as NE-213.

3. 5-in BB horizontal traverse at $30 \mathrm{~cm}$ behind configuration.

4. Bare, ca-covered, 3-, 5-, 8-, 10-in BB on centerline:
a. at $30 \mathrm{~cm}$ behind configuration
b. at $304.8 \mathrm{~cm}$ from reactor centerline (Foreground and Background) .

5. Bare detector on centerline at $30 \mathrm{~cm}$ behind configuration inside partial cadmium enclosure that surrounded the detector except on the side of the last slab in the configuration.

6. Bare detector on centerline at $304.8 \mathrm{~cm}$ point with cadmium sheet over face of last slab in the configuration.

7. INS ${ }^{3} \mathrm{He} \mathrm{BB}$ measurements on centerline: 
a. at $30 \mathrm{~cm}$ behind configuration

b. at $304.8 \mathrm{~cm}$ from reactor centerline (Foreground and Background).

B. SMl $+1.27 \mathrm{~cm} \mathrm{Al}+15.24 \mathrm{~cm}$ SS $+20.32 \mathrm{~cm}$ graphite

1. 5-in $\mathrm{BB}$ horizontal traverse at $30 \mathrm{~cm}$ behind configuration.

2. Bare, od-covered, 3-, 5-, 8-, 10-in BB on centerline:

a. at $30 \mathrm{~cm}$ behind configuration

b. at $304.8 \mathrm{~cm}$ from reactor centerline (Foreground and Background).

3. Bare detector on centerline at $30 \mathrm{~cm}$ behind configuration inside partial cadmium enclosure that surrounded the detector except on the side of the last slab in the configuration.

4. Bare detector on centerline at $304.8 \mathrm{~cm}$ point with cadmium sheet over face of last slab in the configuration.

C. SMI $+1.27 \mathrm{~cm} \mathrm{Al}+15.24 \mathrm{~cm} \mathrm{SS}+30.48 \mathrm{~cm}$ graphite

1. Bare, od-covered, 3-, 5-, 8-, 10-in BB on centerline:

a. at $30 \mathrm{~cm}$ behind configuration

b. at $304.8 \mathrm{~cm}$ from reactor centerline (Foreground and Background).

2. Bare detector on centerline at $30 \mathrm{~cm}$ behind configuration inside partial cadmium enclosure that surrounded the detector except on the side of the last slab in the configuration.

3. Bare detector on centerline at $304.8 \mathrm{~cm}$ point with cadmium sheet over face of last slab in the configuration.

D. SMI + $1.27 \mathrm{~cm} \mathrm{Al}+15.24 \mathrm{~cm} \mathrm{SS}+40.64 \mathrm{~cm}$ graphite

1. 5-in BB horizontal traverse at $30 \mathrm{~cm}$ behind configuration.

2. Bare, cd-covered, 3-, 5-, 8-, 10-in BB on centerline:

a. at $30 \mathrm{~cm}$ behind configuration

b. at $304.8 \mathrm{~cm}$ from reactor centerline (Foreground and Background). 
3. Bare detector on centerline at $30 \mathrm{~cm}$ behind configuration inside partial cadmium enclosure that surrounded the detector except on the side of the last slab in the configuration.

4. Bare detector on centerline at $304.8 \mathrm{~cm}$ point with cadmium sheet over face of last slab in the configuration.

5. INS ${ }^{3} \mathrm{He} \mathrm{BB}$ measurements on centerline:
a. at $30 \mathrm{~cm}$ behind configuration
b. at $304.8 \mathrm{~cm}$ from reactor centerline (Foreground and Background).

E. $\mathrm{SMI}+1.27 \mathrm{~cm} \mathrm{Al}+15.24 \mathrm{~cm} \mathrm{SS}+50.8 \mathrm{~cm}$ graphite

1. Bare, cd-covered, 3-, 5-, 8-, 10-in BB on centerline:
a. at $30 \mathrm{~cm}$ behind configuration
b. at $304.8 \mathrm{~cm}$ from reactor centerline (Foreground and Background).

2. Bare detector on centerline at $30 \mathrm{~cm}$ behind configuration inside partial cadmium enclosure that surroumded the detector except on the side of the last slab in the configuration.

3. Bare detector on centerline at $304.8 \mathrm{~cm}$ point with cadmium sheet over face of last slab in the configuration.

F. . SMI + $1.27 \mathrm{~cm} \mathrm{Al}+15.24 \mathrm{~cm} \mathrm{SS}+60.96 \mathrm{~cm}$ graphite

1. NE-213 and hydrogen counter measurements on centerline as close as feasible behind ss.

2. 3-, 5-, 10-in BB measurements on centerline at same location as NE-213.

3. 5-in $\mathrm{BB}$ horizontal traverse at $30 \mathrm{~cm}$ behind configuration.

4. Bare, cd-covered, 3-, 5-, 8-, 10-in BB on centerline:
a. at $30 \mathrm{~cm}$ behind configuration
b. at $304.8 \mathrm{~cm}$ from reactor centerline (Foreground and Background) .

5. Bare detector on centerline at $30 \mathrm{~cm}$ behind configuration inside partial cadmium enclosure that surrounded the detector except on the side of the last slab in the configuration. 
6. Bare detector on centerline at $304.8 \mathrm{~cm}$ point with cadmium sheet over face of last slab in the configuration.

7. INS ${ }^{3} \mathrm{He} \mathrm{BB}$ measurements on centerline:

a. at $30 \mathrm{~cm}$ behind configuration

b. at $304.8 \mathrm{~cm}$ from reactor centerline (Foreground and Background).

VII. SM + SS and $B_{4} C$ (near core shield)

A. SMl $(10.27 \mathrm{~cm} \mathrm{Fe}+9.17 \mathrm{~cm} \mathrm{Al}+2.54 \mathrm{~cm}$ boral $+20.32 \mathrm{~cm}$ radial

blanket) $+1.27 \mathrm{~cm} \mathrm{Al}+15.24 \mathrm{~cm} \mathrm{SS}+1.27 \mathrm{~cm} \mathrm{Al}+15.24 \mathrm{~cm} \mathrm{SS}$

1. 5-in BB horizontal traverse at $30 \mathrm{~cm}$ behind configuration.

2. 3-, 5-, 8-, 10-in BB measurements on centerline:

a. at $30 \mathrm{~cm}$ behind configuration

b. at $304.8 \mathrm{~cm}$ from reactor centerline (Foreground and Background).

B. $\mathrm{SMI}+1.27 \mathrm{~cm} \mathrm{Al}+15.24 \mathrm{~cm} \mathrm{SS}+1.27 \mathrm{~cm} \mathrm{Al}+15.24 \mathrm{~cm} \mathrm{SS}+1.27 \mathrm{~cm} \mathrm{Al}$ $+15.24 \mathrm{~cm}$ SS

1. 5-in $\mathrm{BB}$ horizontal traverse at $30 \mathrm{~cm}$ behind configuration.

2. 3-, 5-, 8-, 10-in $\mathrm{BB}$ measurements on centerline:

a. at $30 \mathrm{~cm}$ behind configuration

b. at $304.8 \mathrm{~cm}$ from reactor centerline (Foreground and Background).

C. $\mathrm{SMI}+1.27 \mathrm{~cm} \mathrm{Al}+15.24 \mathrm{~cm} \mathrm{SS}+1.27 \mathrm{~cm} \mathrm{Al}+15.24 \mathrm{~cm} \mathrm{SS}+1.27 \mathrm{~cm} \mathrm{Al}$ $+15.24 \mathrm{~cm} \mathrm{SS}+1.27 \mathrm{~cm} \mathrm{Al}+15.24 \mathrm{~cm} \mathrm{~B} \mathrm{~B}_{4}+2.54 \mathrm{~cm} \mathrm{SS}$

1. 5-in BB horizontal traverse at $30 \mathrm{~cm}$ behind configuration.

2. 3-, 5-, 8-, 10-in BB measurements on centerline:

a. at $30 \mathrm{~cm}$ behind configuration

b. at $304.8 \mathrm{~cm}$ from reactor centerline (Foreground and Background) .

3. TNS ${ }^{3} \mathrm{He}$ BB measurements on centerline:

a. at $30 \mathrm{~cm}$ behind configuration 
b. at $304.8 \mathrm{~cm}$ from reactor centerline (Foreground and Background).

D. $\mathrm{SMI}+1.27 \mathrm{~cm} \mathrm{Al}+15.24 \mathrm{~cm} \mathrm{SS}+1.27 \mathrm{~cm} \mathrm{Al}+15.24 \mathrm{~cm} \mathrm{SS}+1.27 \mathrm{~cm} \mathrm{Al}$

$+15.24 \mathrm{~cm} \mathrm{SS}+1.27 \mathrm{~cm} \mathrm{Al}+15.24 \mathrm{~cm} \mathrm{~B} \mathrm{~B}_{4} \mathrm{C}+1.54 \mathrm{~cm} \mathrm{SS}+30.48 \mathrm{~cm} \mathrm{Na}$

1. Bare, od-covered, 3-, 5-, 8-, 10-in BB measurements on centerline:

a. at $30 \mathrm{~cm}$ behind configuration

b. at $304.8 \mathrm{~cm}$ from reactor centerline (Foreground and Background).

2. Bare detector on centerline at $30 \mathrm{~cm}$ behind configuration inside partial cadmium enclosure that surrounded the detector except on the side of the last slab in the configuration.

3. Bare detector on centerline at $304.8 \mathrm{~cm}$ point with cadmium shell over face of last slab in the configuration. 

APPENDIX B

EXPERTMENTAL CONFIGURATIONS 


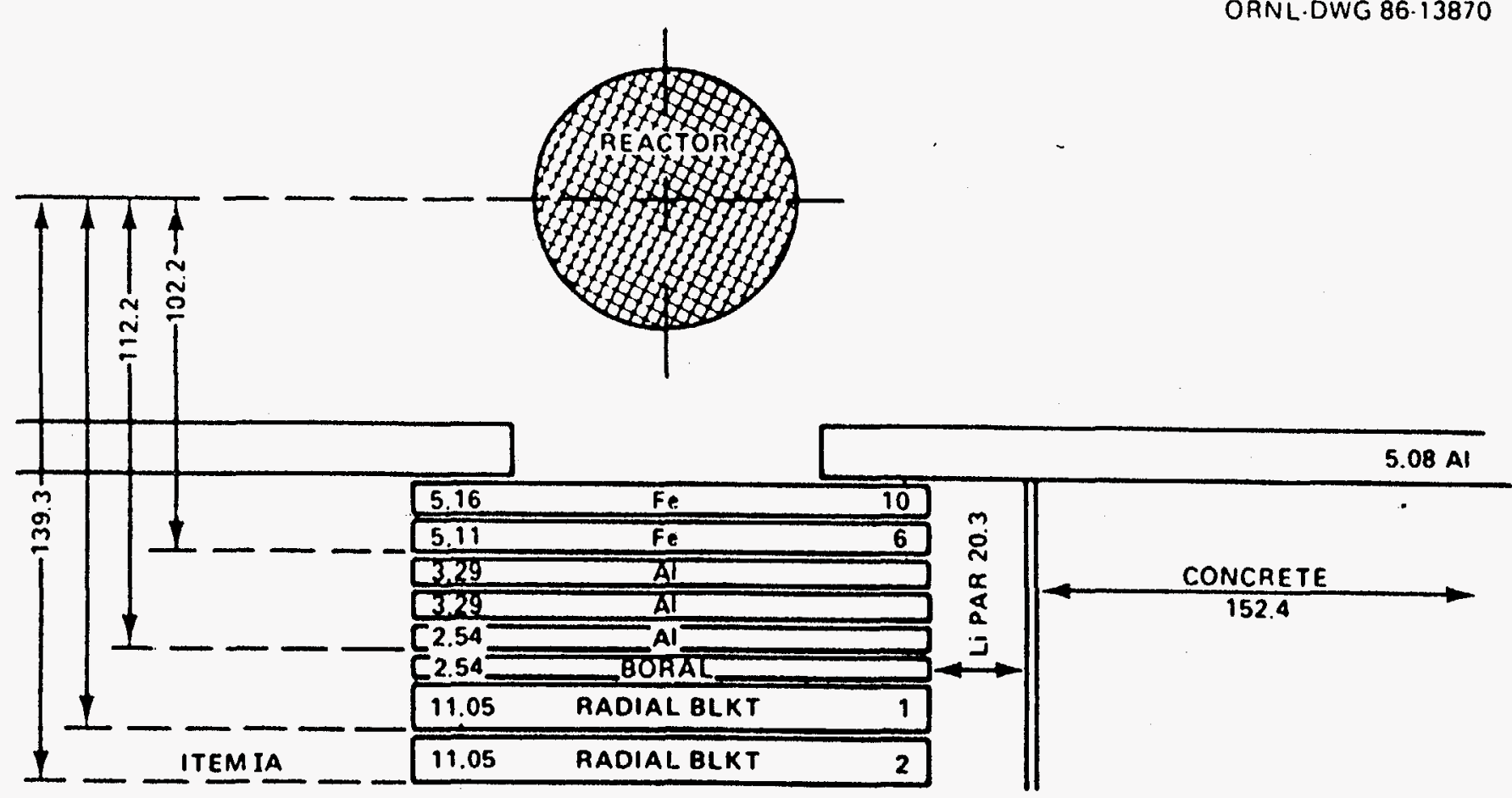

Figure Bl. Sketch of the configuration for measurements under section I.A of the experimental program plan. 


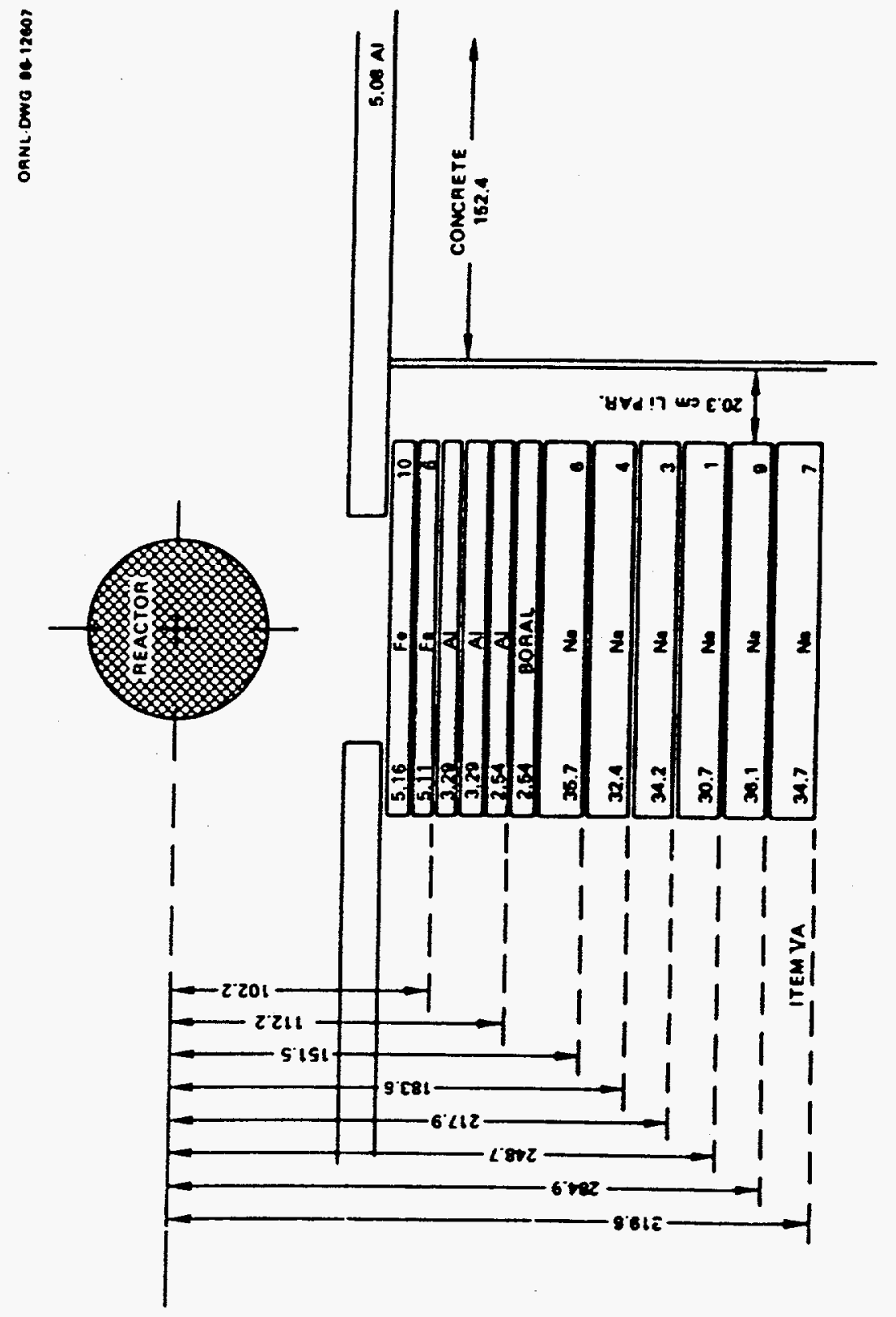

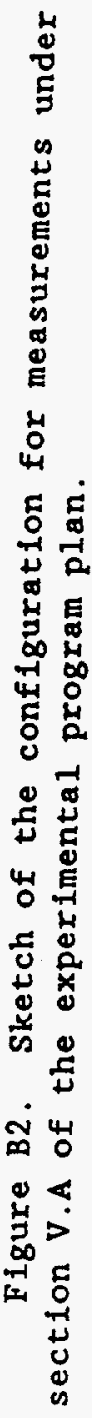


0
$\mathbf{m}$
0
0
0
0
$\vdots$
0
$\vdots$
0
0
0
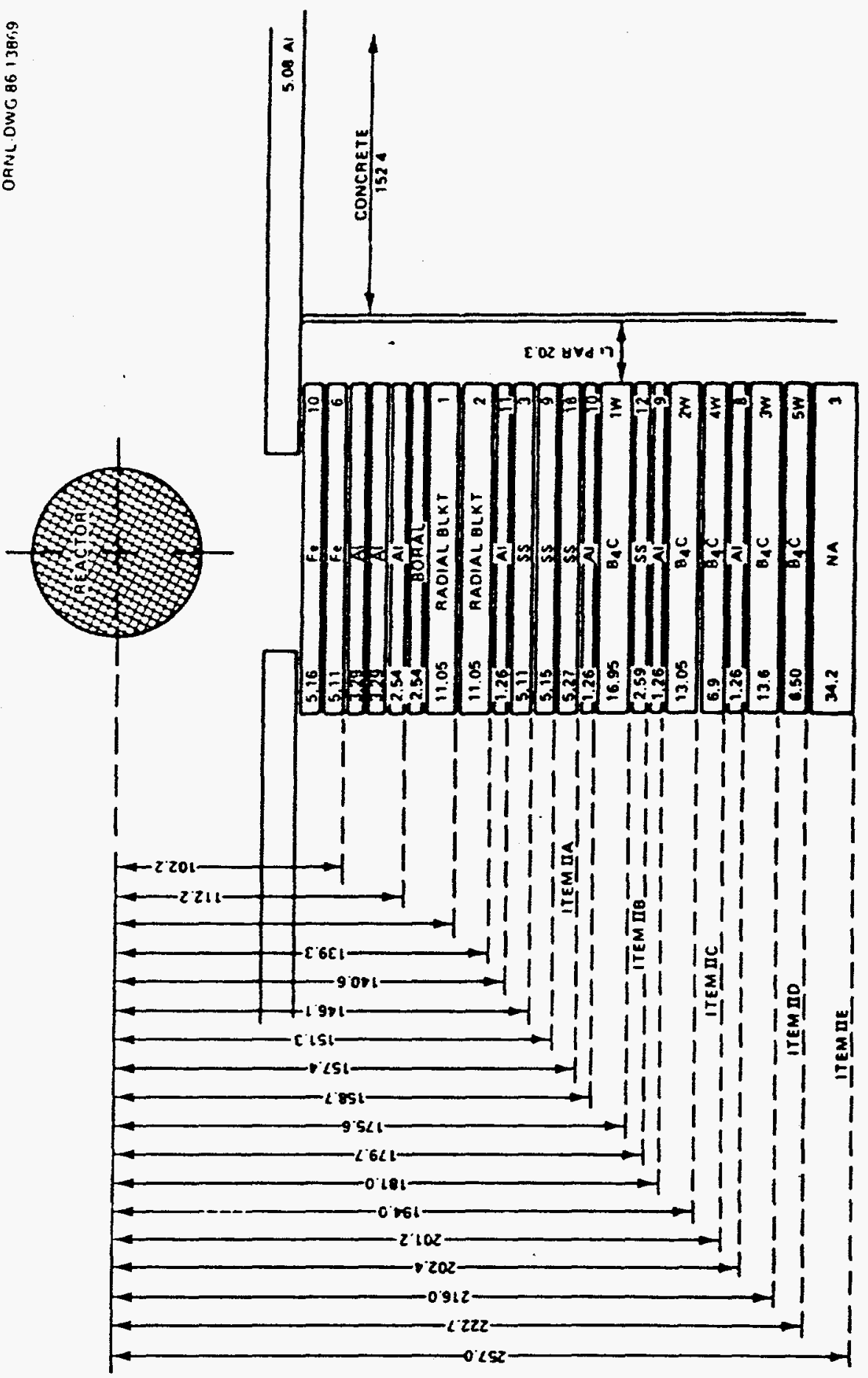

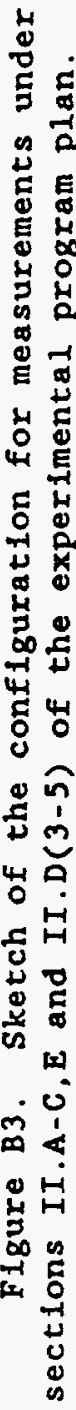




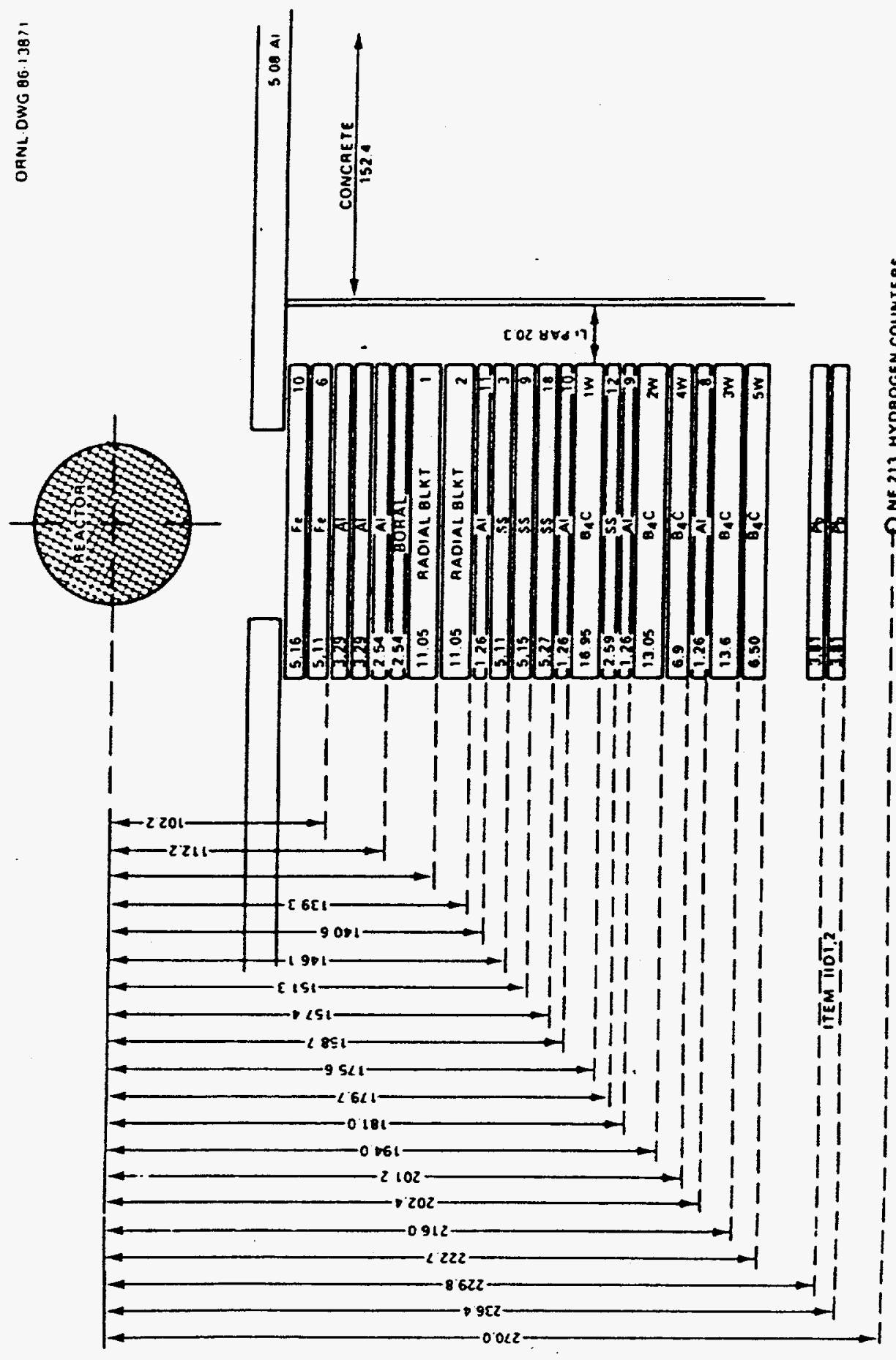

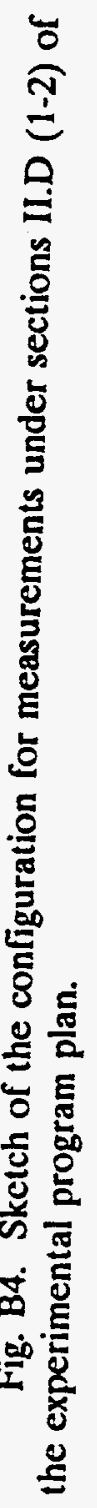




\section{B,C CONTAINERS}
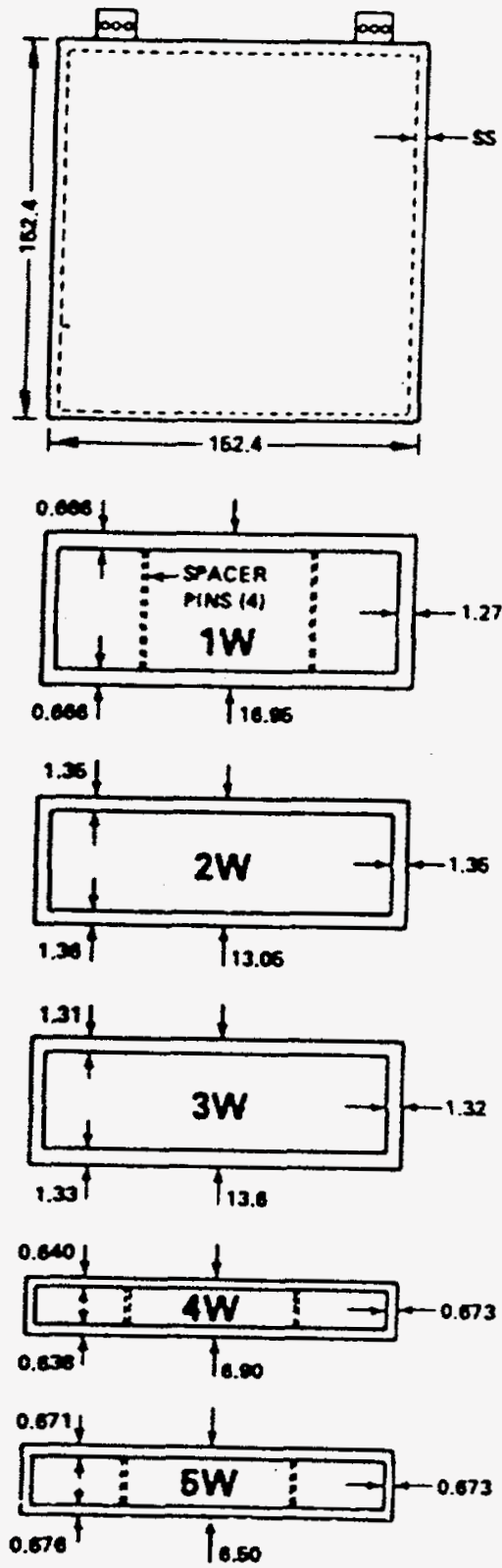

IALL DIMENSIONS ARE IN CENTIMETERS

Figure B5. Sketches of the stainless steel containers used to form the $B_{4} C$ shield slabs. 


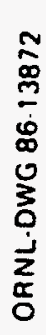
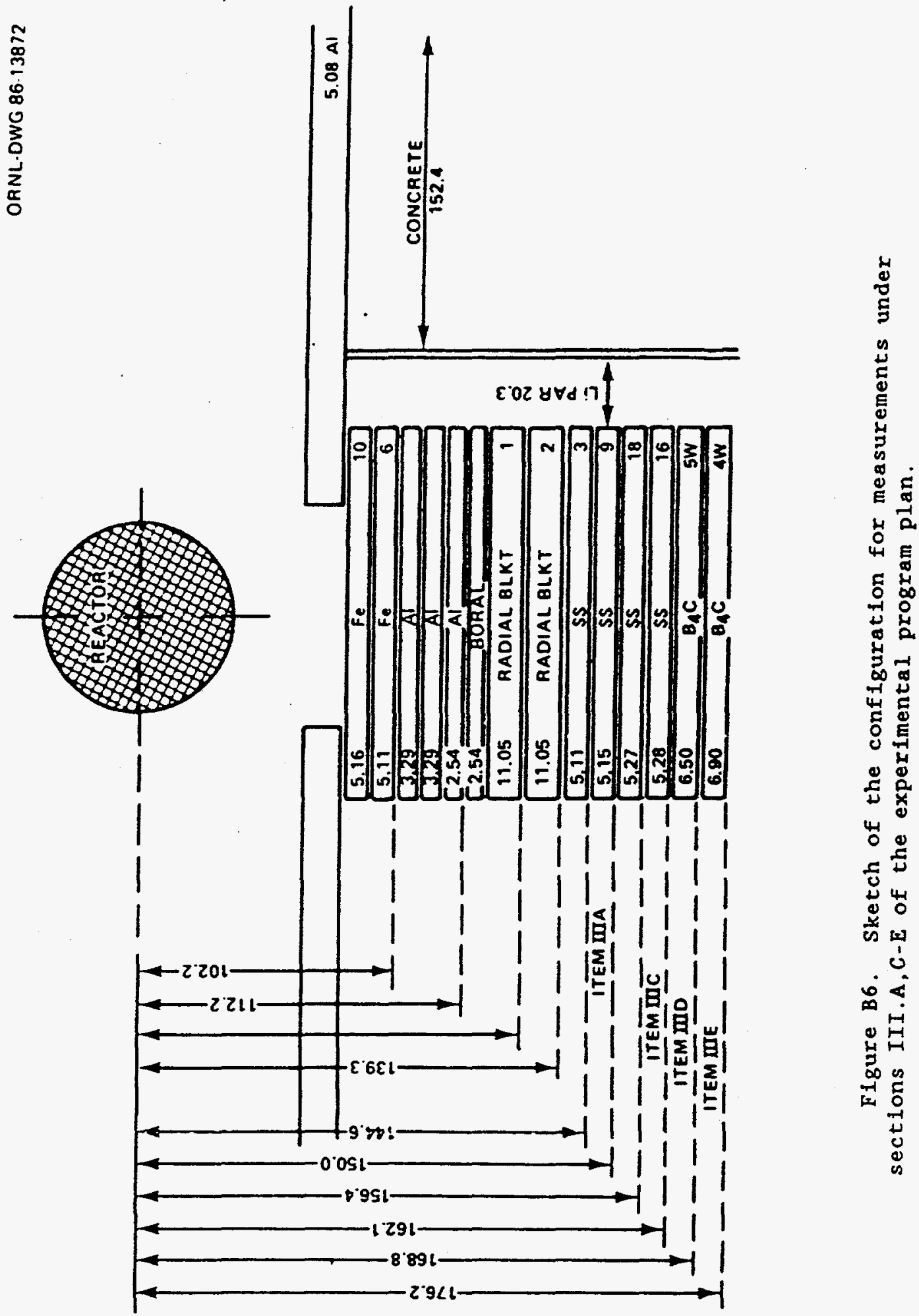

年 
$m$
0
0
$⿱$
$\dot{0}$
0
0
$\vdots$
0
$\vdots$
$\vdots$
0
0
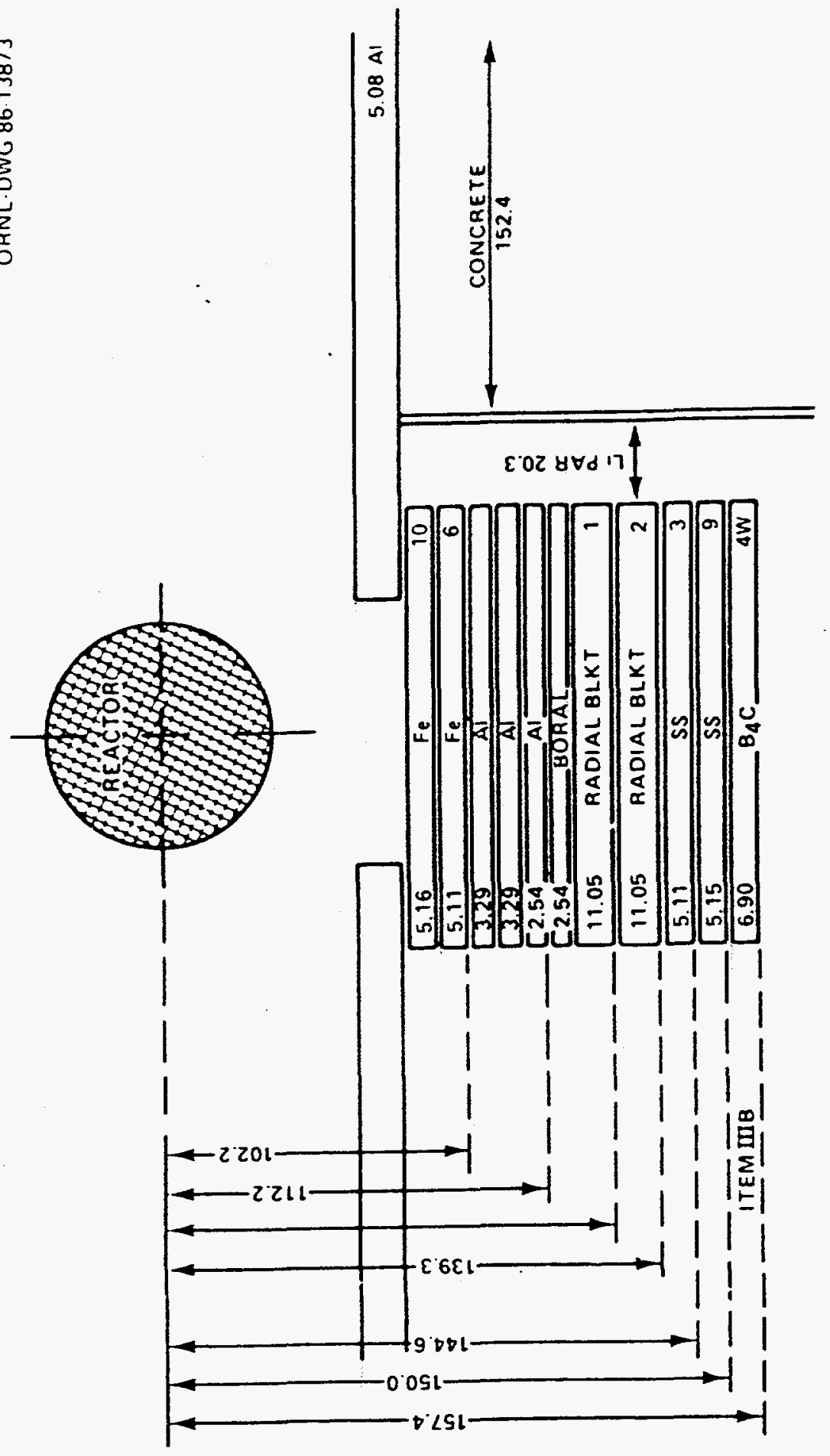

峁



के

동

号

苟

0

至

号

岂

H

도용

岁点

出出 
$\sigma$
$\sigma$
$\vdots$
0
$\infty$
0
$\vdots$
0
$\vdots$
$\vdots$
0
0

$m$
$\times$
$m$
0

$N U$
$\times \quad$
$N \quad$

ํㅜㄹ

号

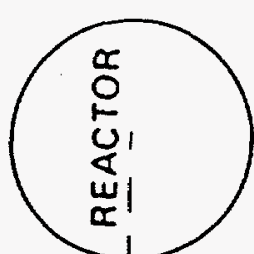

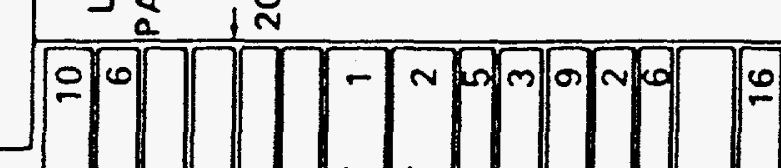

4

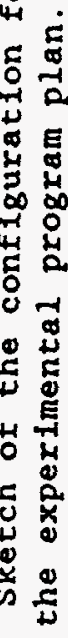

$\infty_{0}^{\infty}$

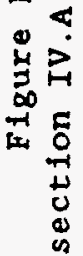


\begin{tabular}{l}
8 \\
\multirow{2}{0}{} \\
$\vdots$ \\
0 \\
0 \\
0 \\
$\vdots$ \\
0 \\
$\vdots$ \\
$z$ \\
0 \\
0
\end{tabular}

$\begin{array}{ll}m \\ \times & 0 \\ m & 0\end{array}$

$\begin{array}{ll}N & 0 \\ \times & 0 \\ N & 0\end{array}$

$\stackrel{\mathscr{D}}{\stackrel{\mathscr{J}}{g}}$

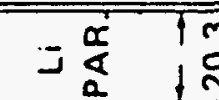

\%

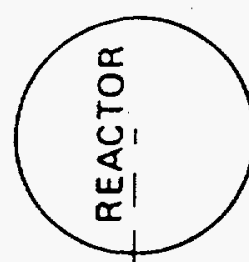

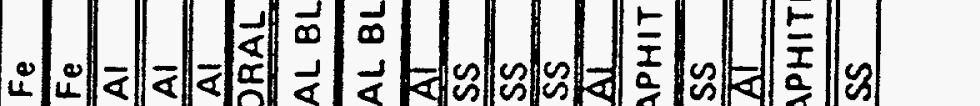

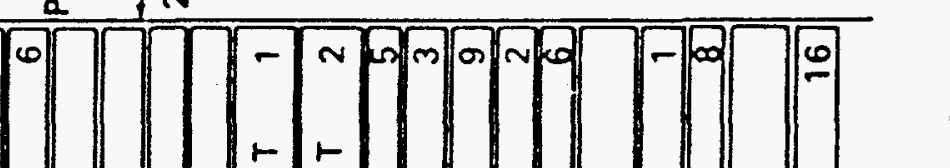

号

بै

동

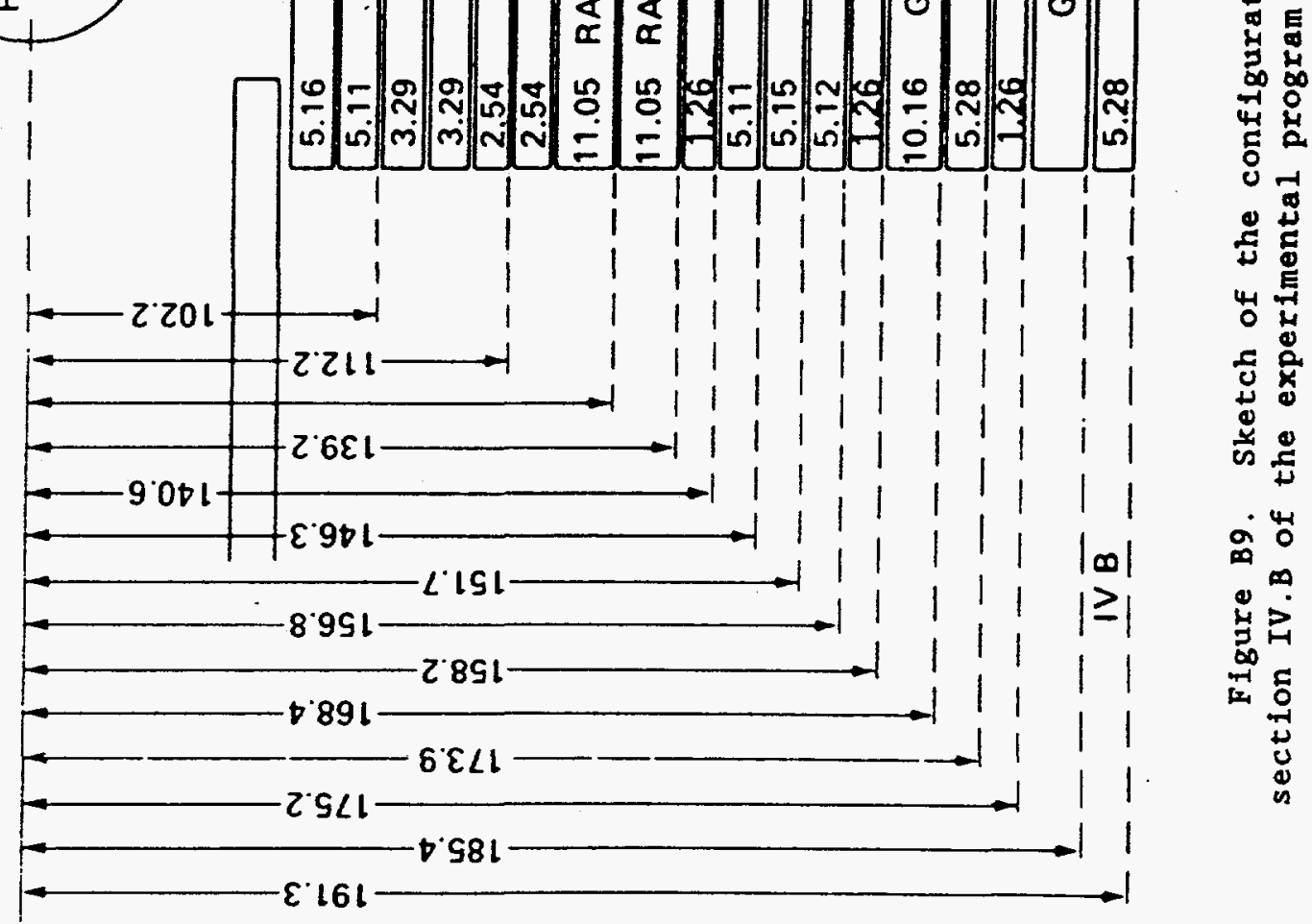




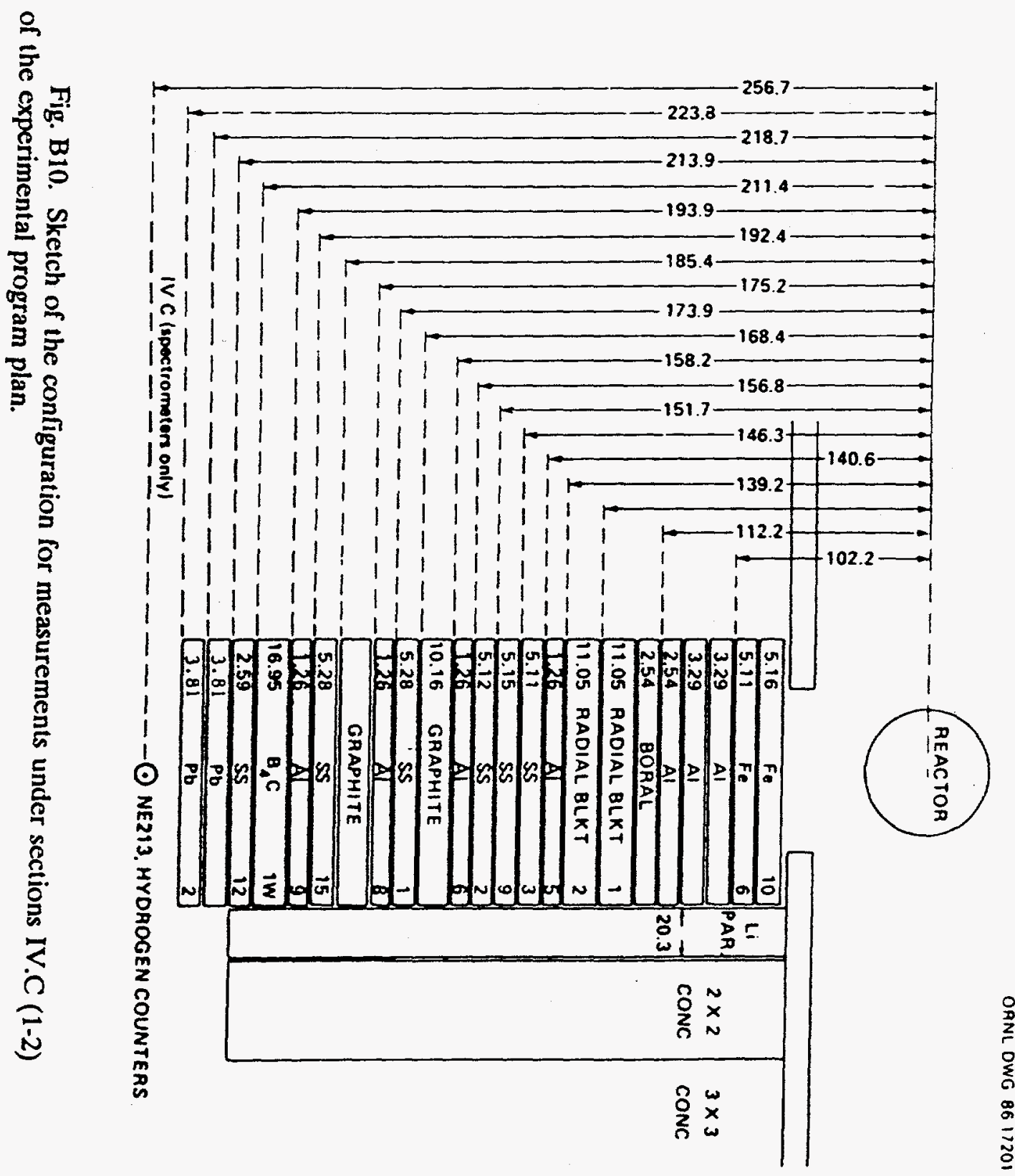




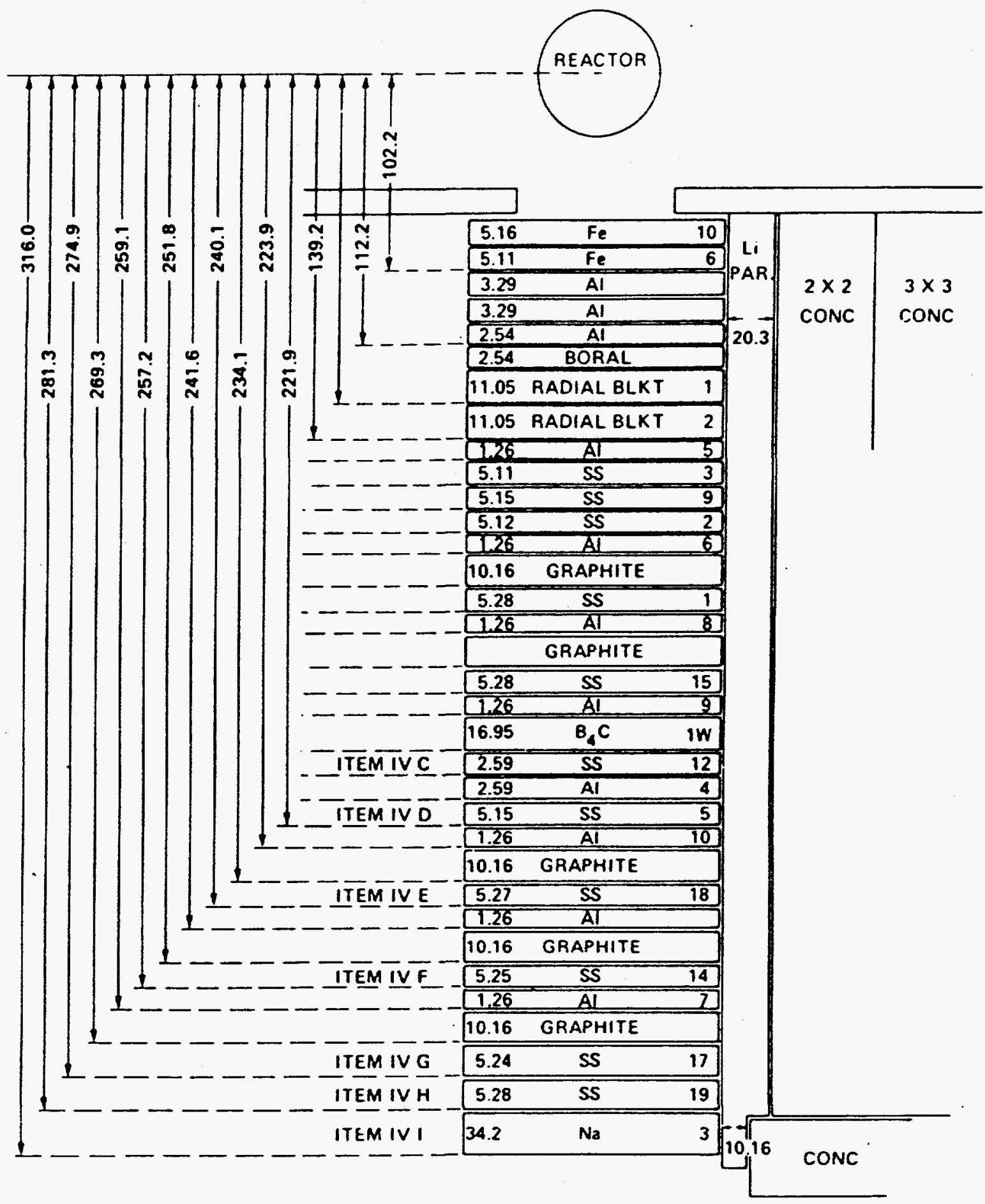

Figure B11. Sketch of the configuration for measurements under sections IV.C(3-5) through IV.I of the experimental program plan. 


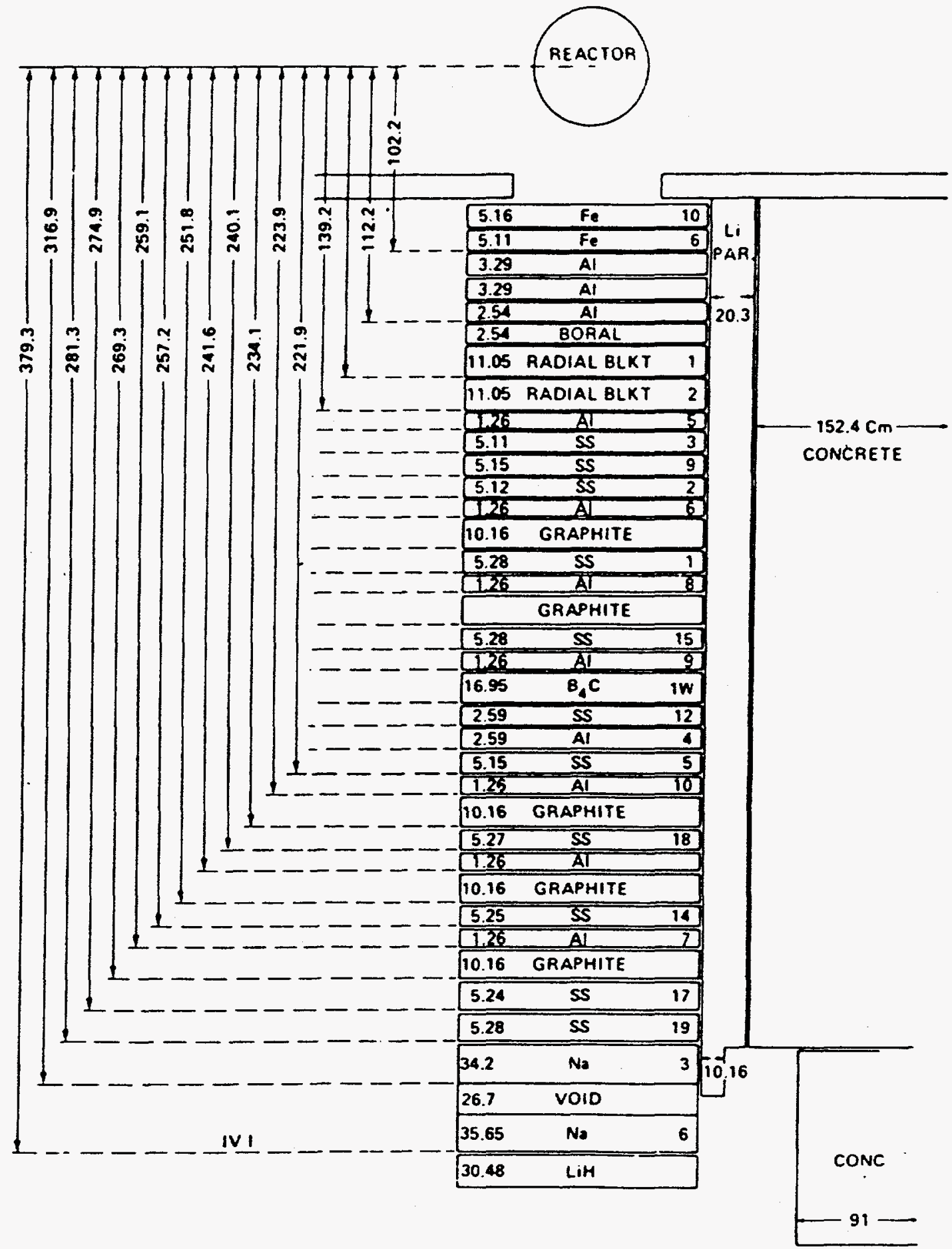

Figure B12. Sketch of the configuration for measurements under section IV. IA of the experimental program plan. 


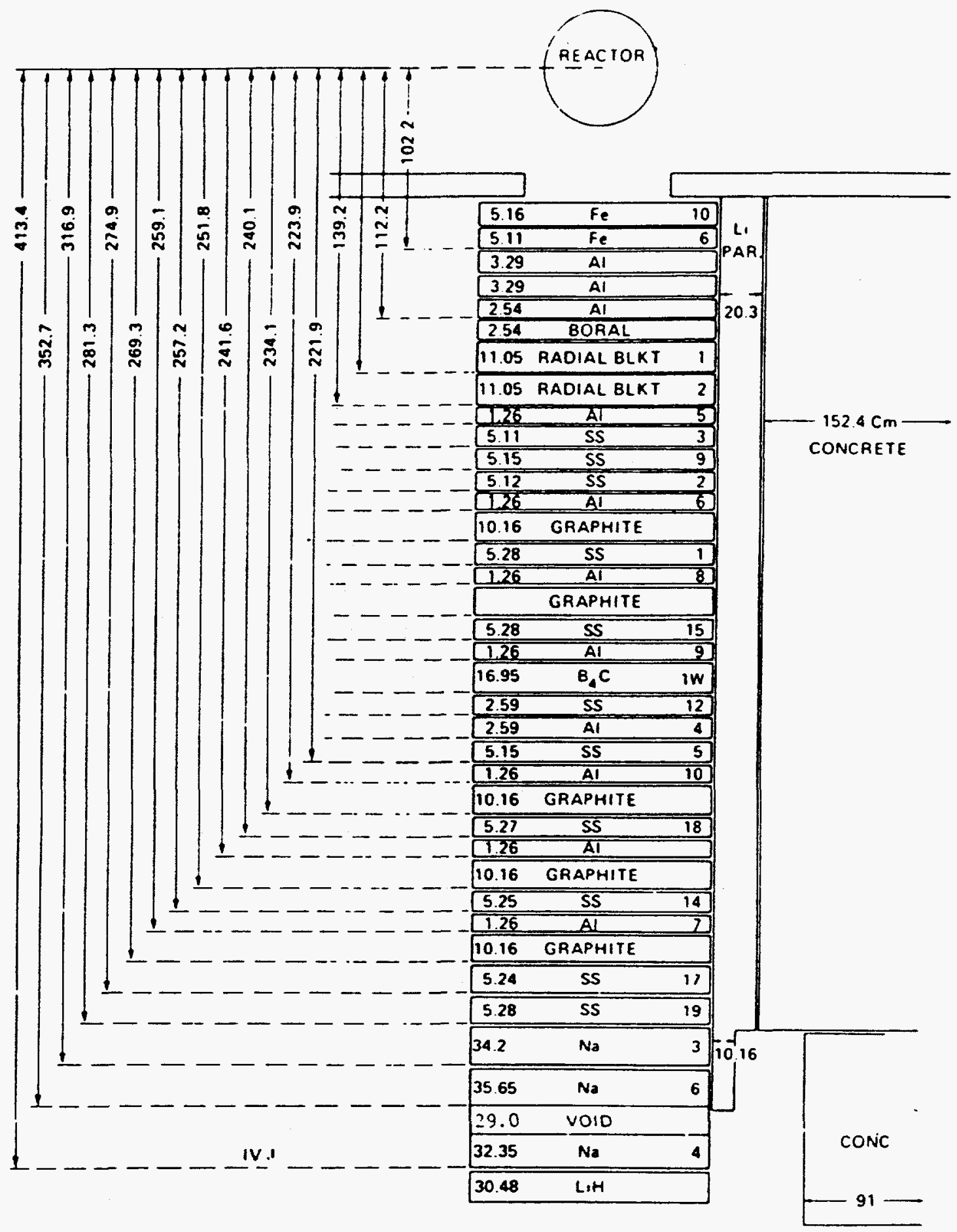

Figure B13. Sketch of the configuration for measurements under section IV.J of the experimental program plan. 
ORNL DWG 86 TIZOA

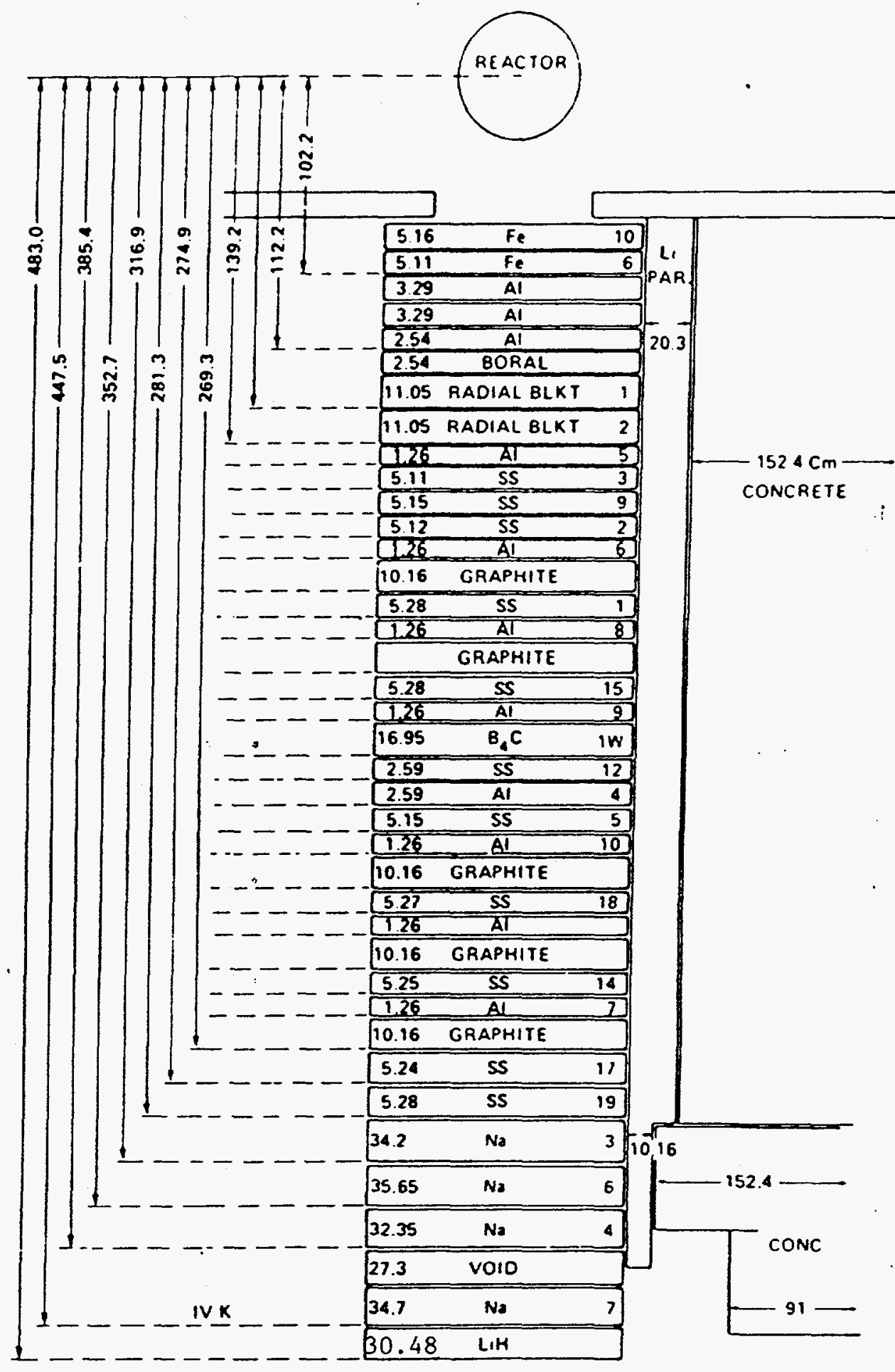

Figure B14. Sketch of the configuration for measurenents under section IV.K of the experimental program plan. 


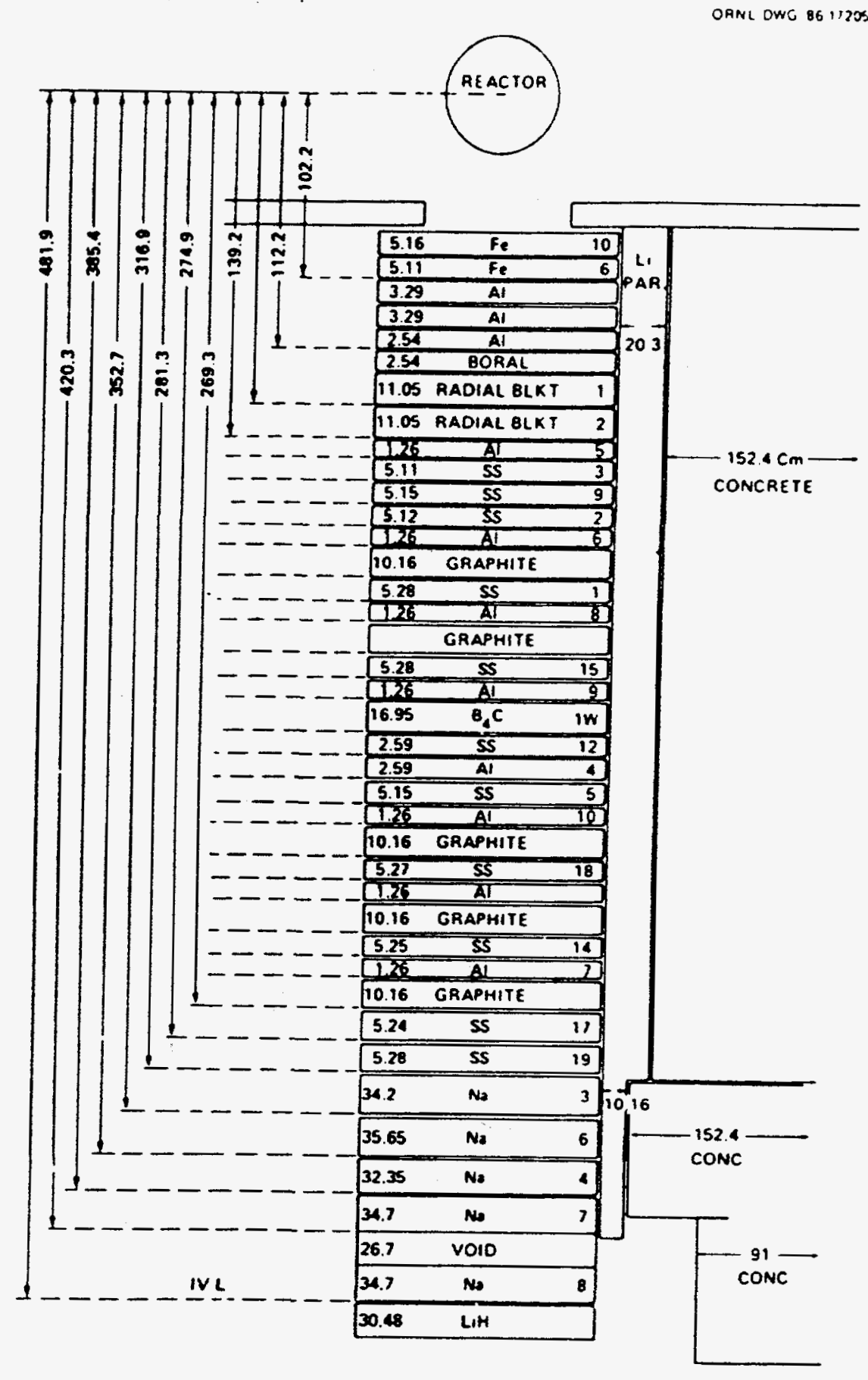

Figure B15. Sketch of the configuration for measurements under section IV.L of the experimental program plan. 


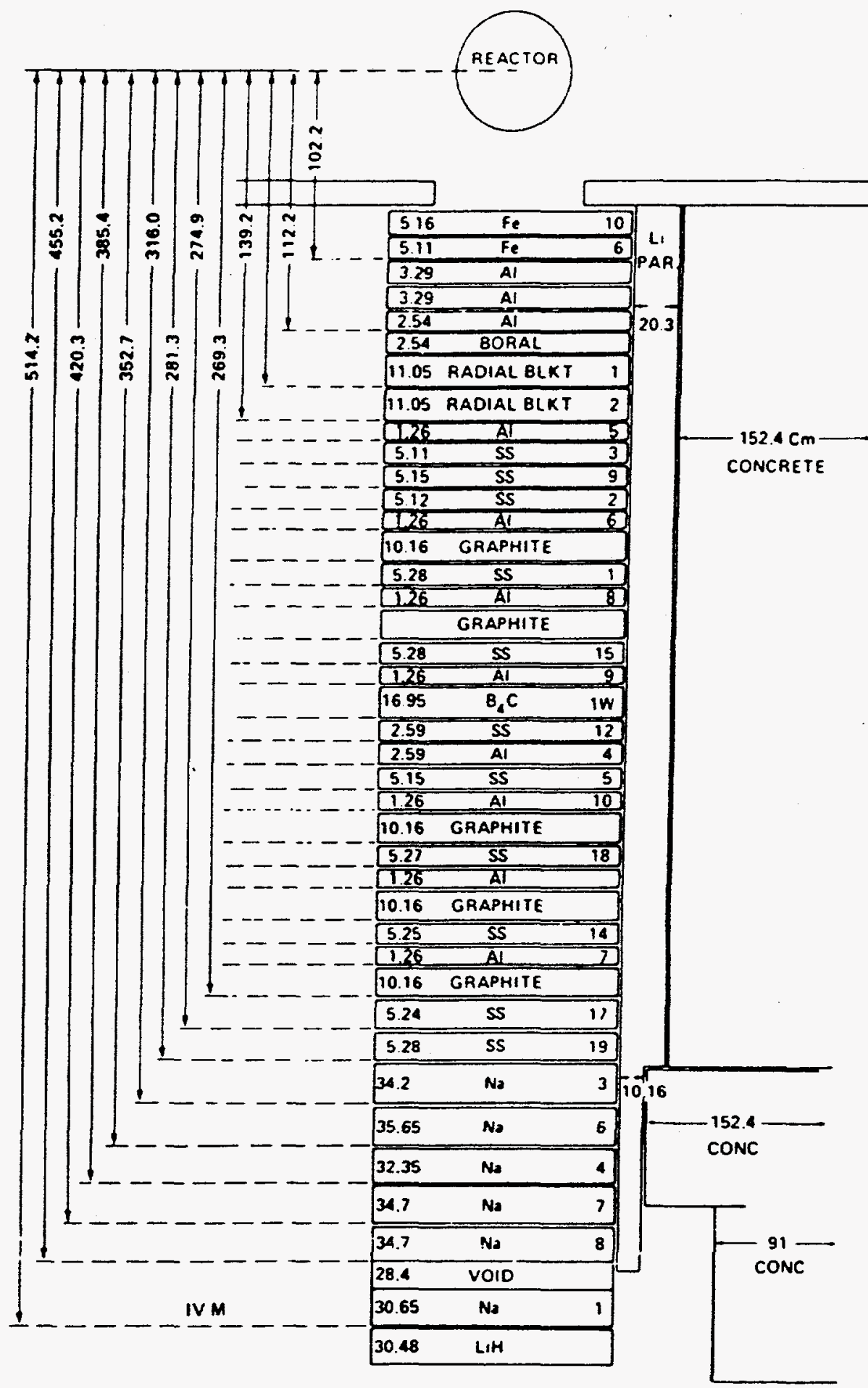

Figure B16. Sketch of the configuration for measurements under section IV.M of the experimental program plan. 


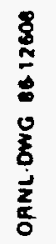

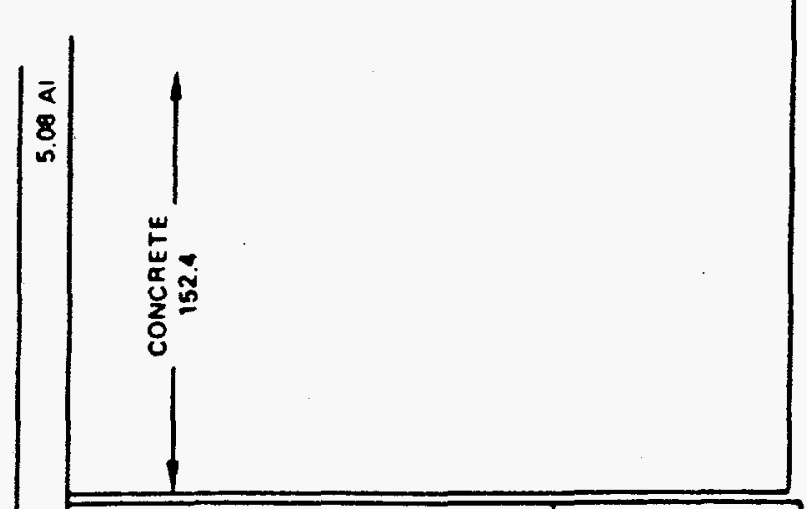

$\stackrel{4}{g}$
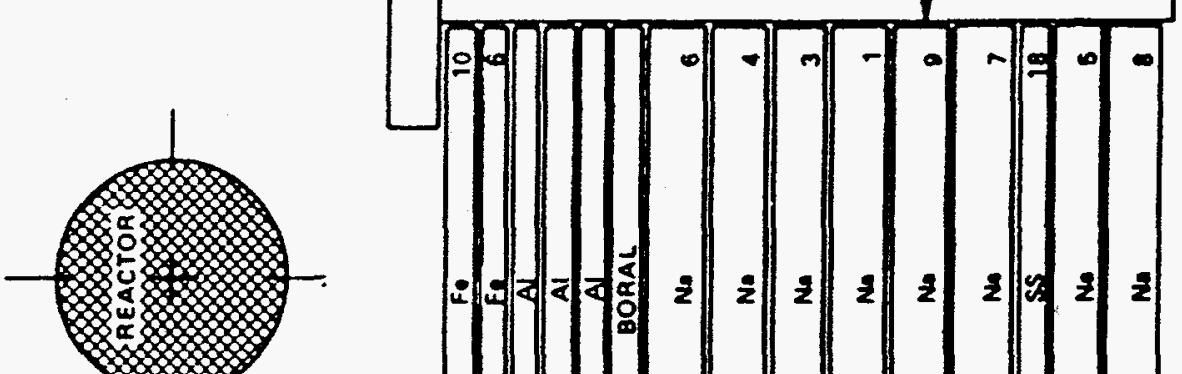

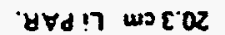

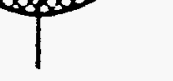

-
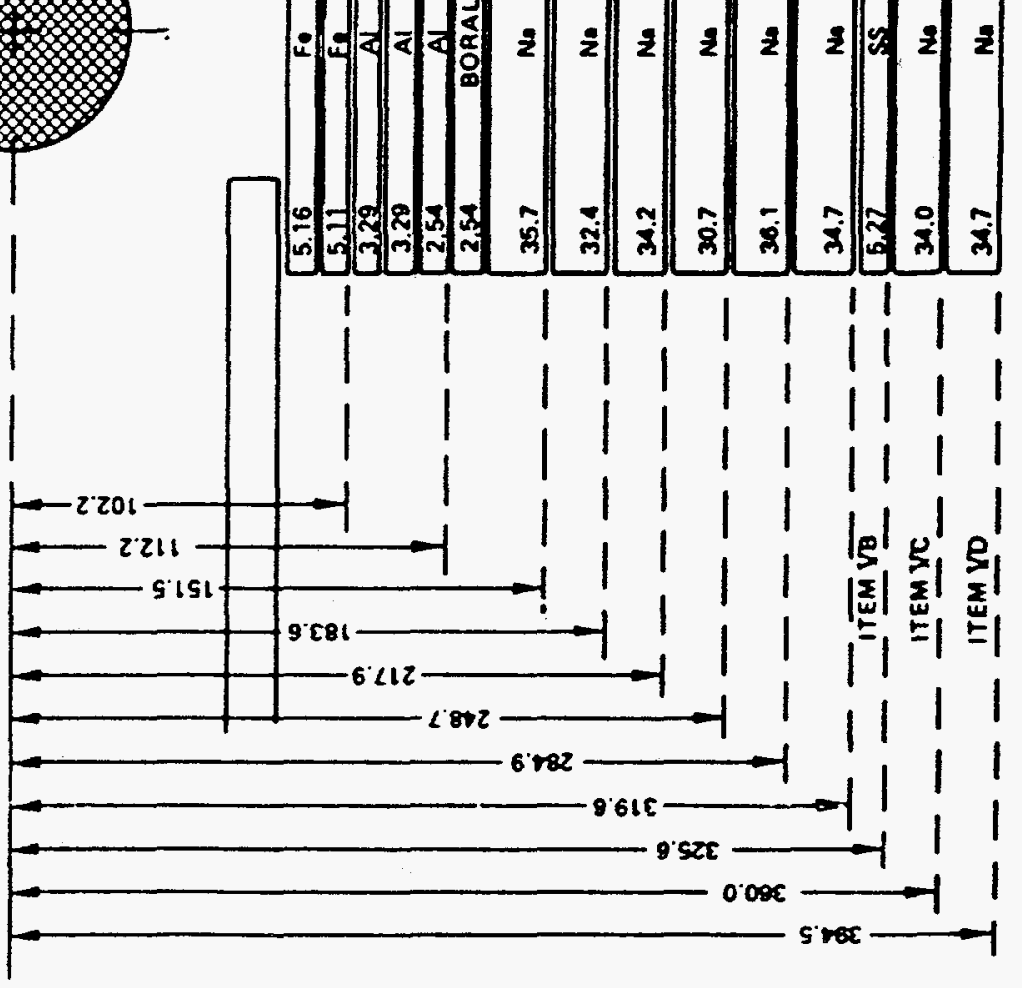

号

年.

동

홍

苛

怘这

ชี

ป 苟

实

岁芩

รี

㟧 $\frac{.}{4}$

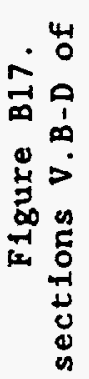




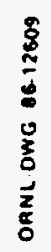
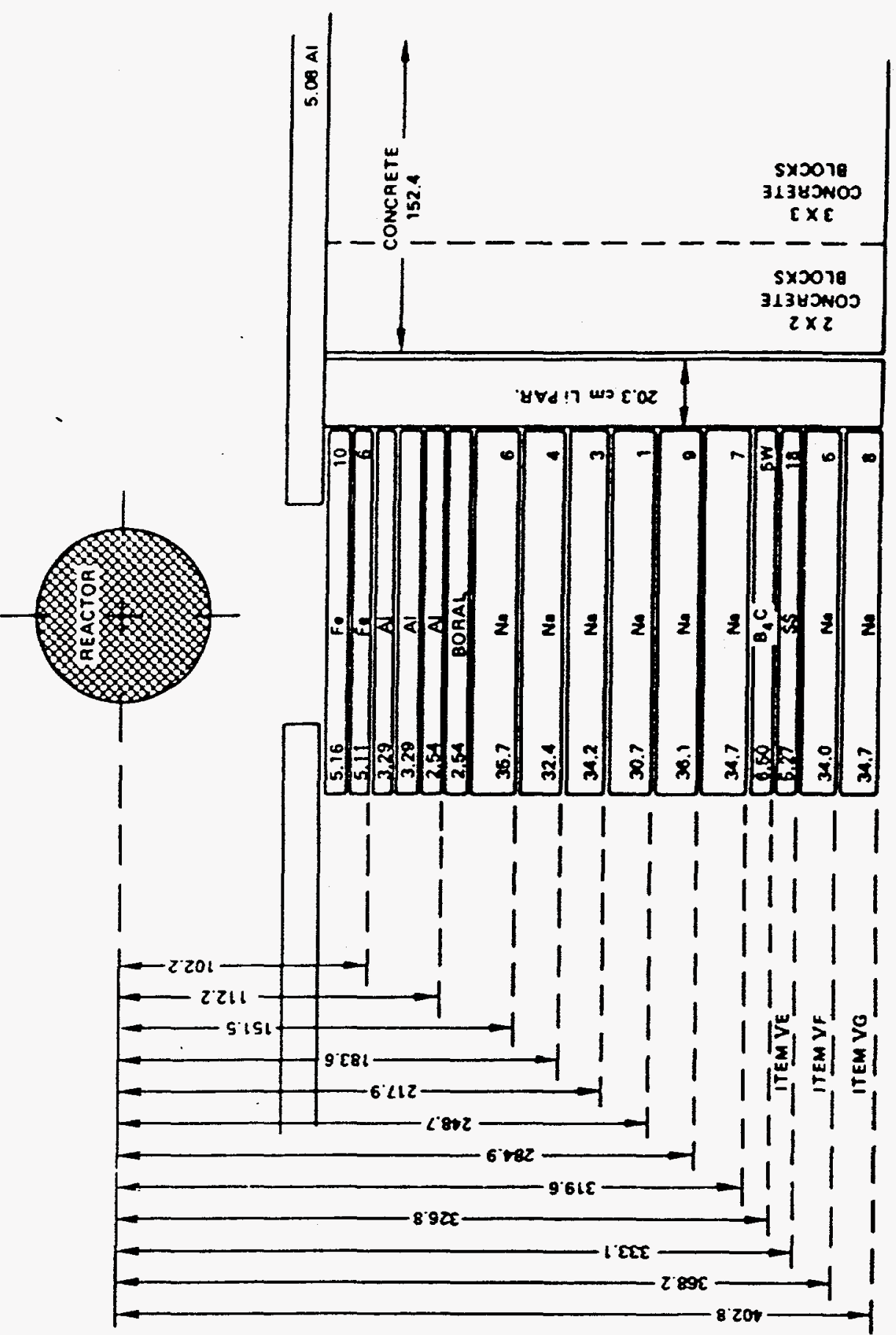

Figure B18. Sketch of the configuration for measurements under sections V.E-G of the experimental program plan. 
0
0
$\frac{1}{8}$
0
0
$\vdots$
$\vdots$
$\frac{2}{2}$
0
0

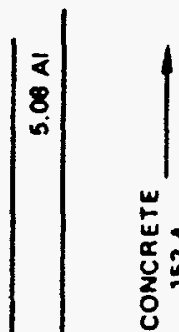
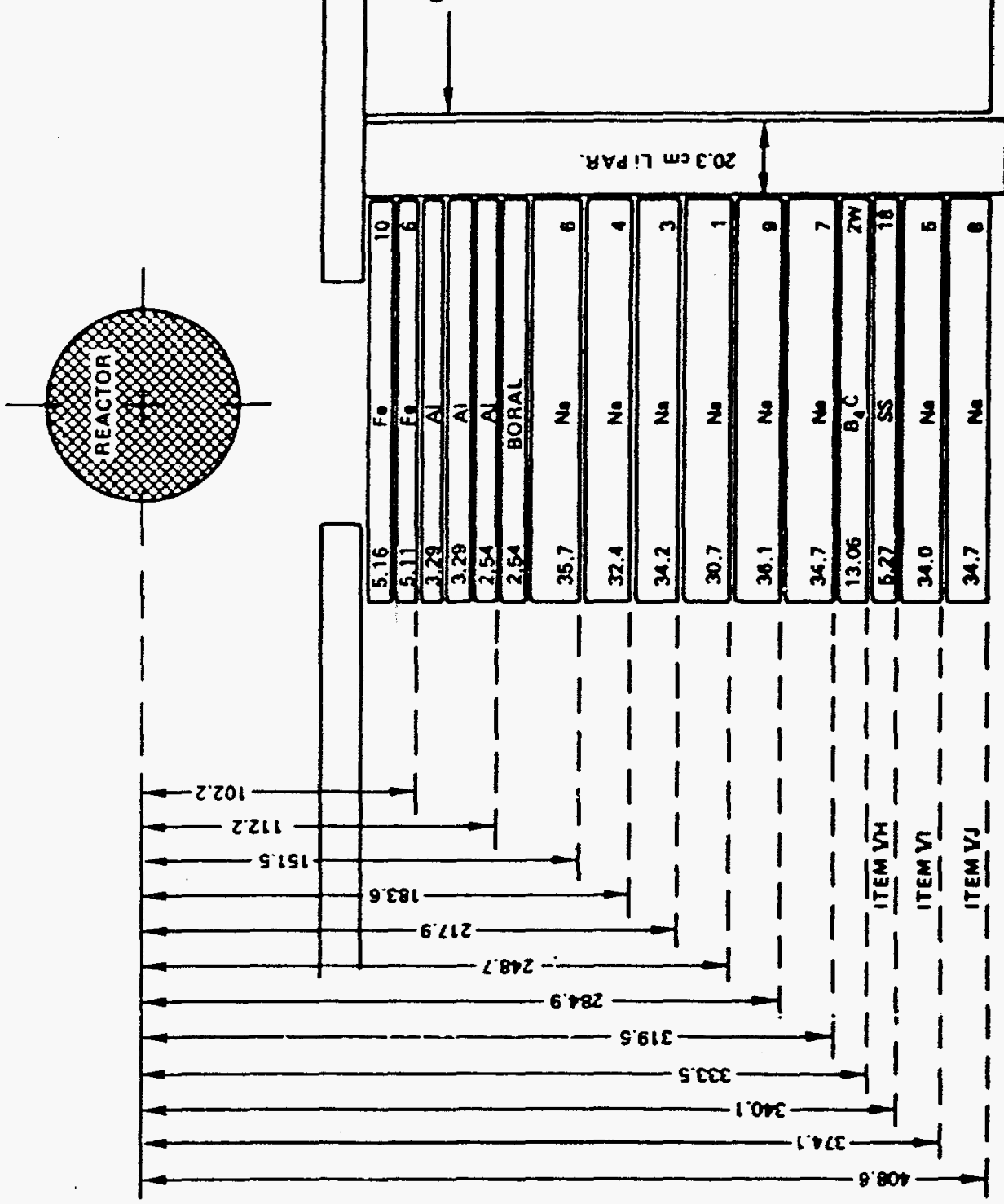

Figure B19. Sketch of the configuration for measurements under sections V.H-J of the experimental program plan. 


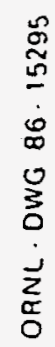

$\begin{array}{ll}m & U \\ \times & 0\end{array}$

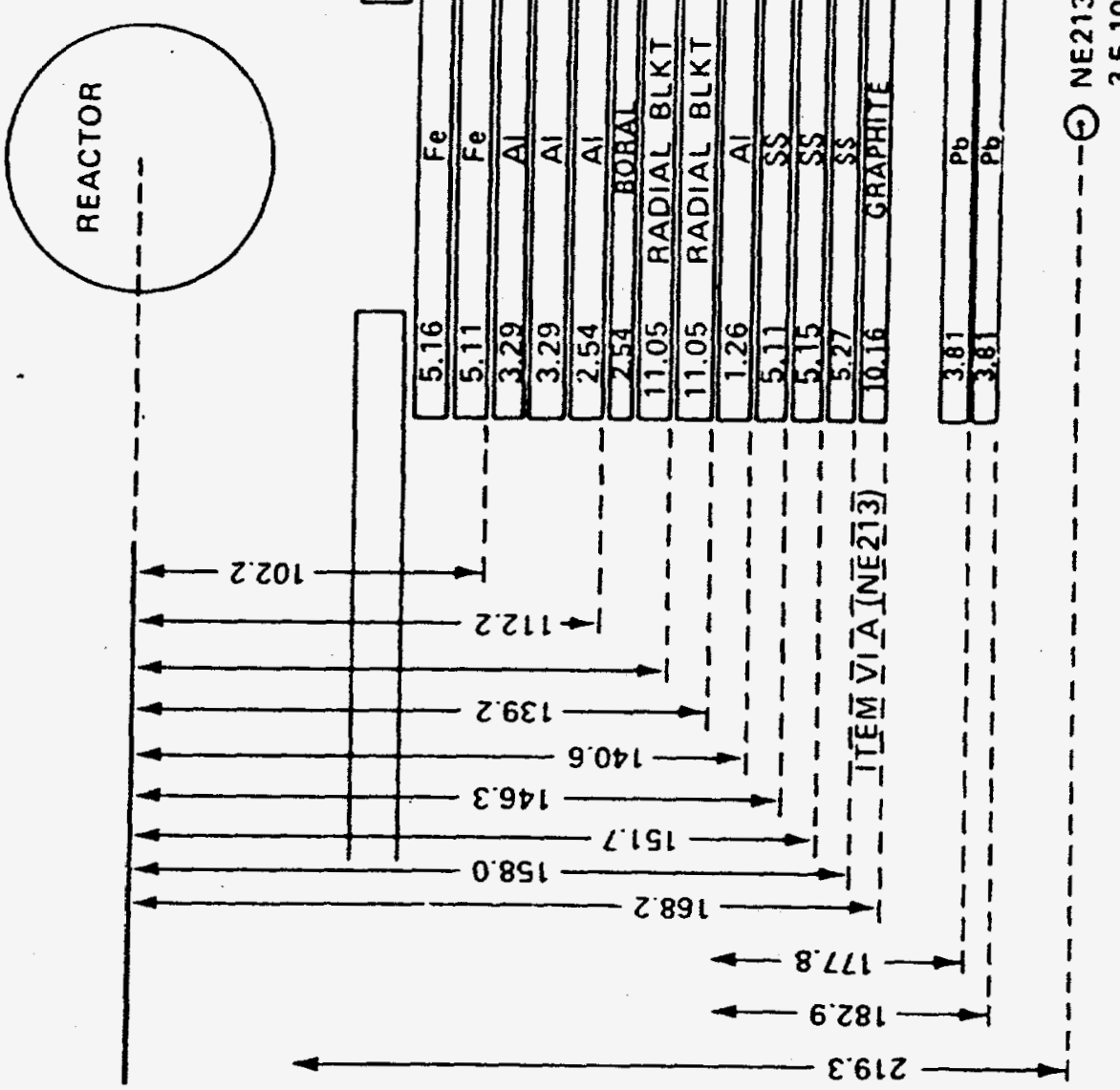

Fig. B20. Sketch of the configuration for measurements under sections IV.A (1-2) of the experimental program plan. 


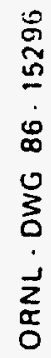

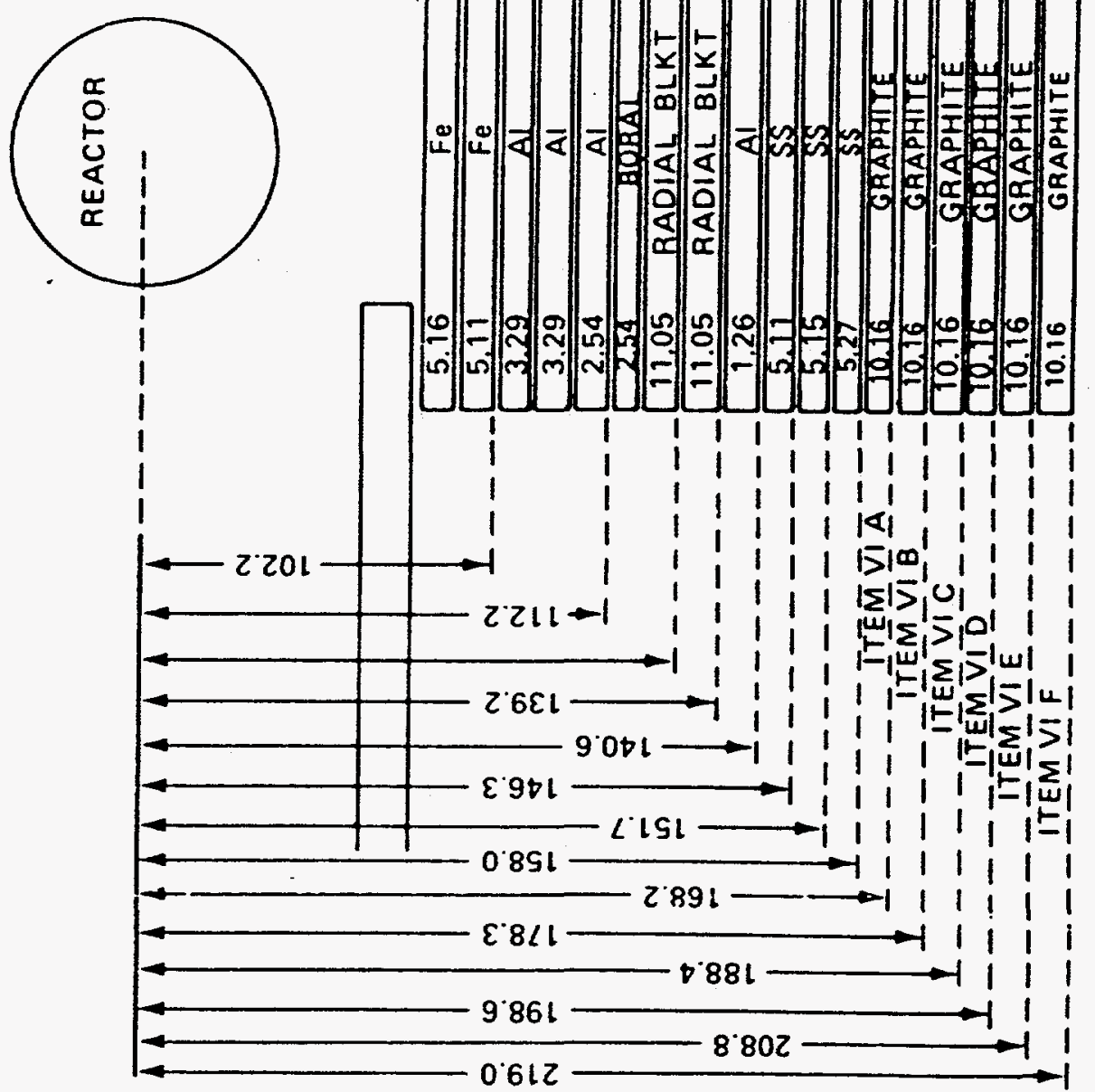

Figure B21. Sketch of the configuration for measurements under sections VI.A(3-5) through VI.E and VI.F(3-5) of the experimental program plan. 


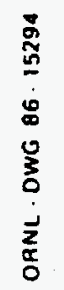$$
\begin{array}{ll}
m & 0 \\
\times & 8
\end{array}
$$

S

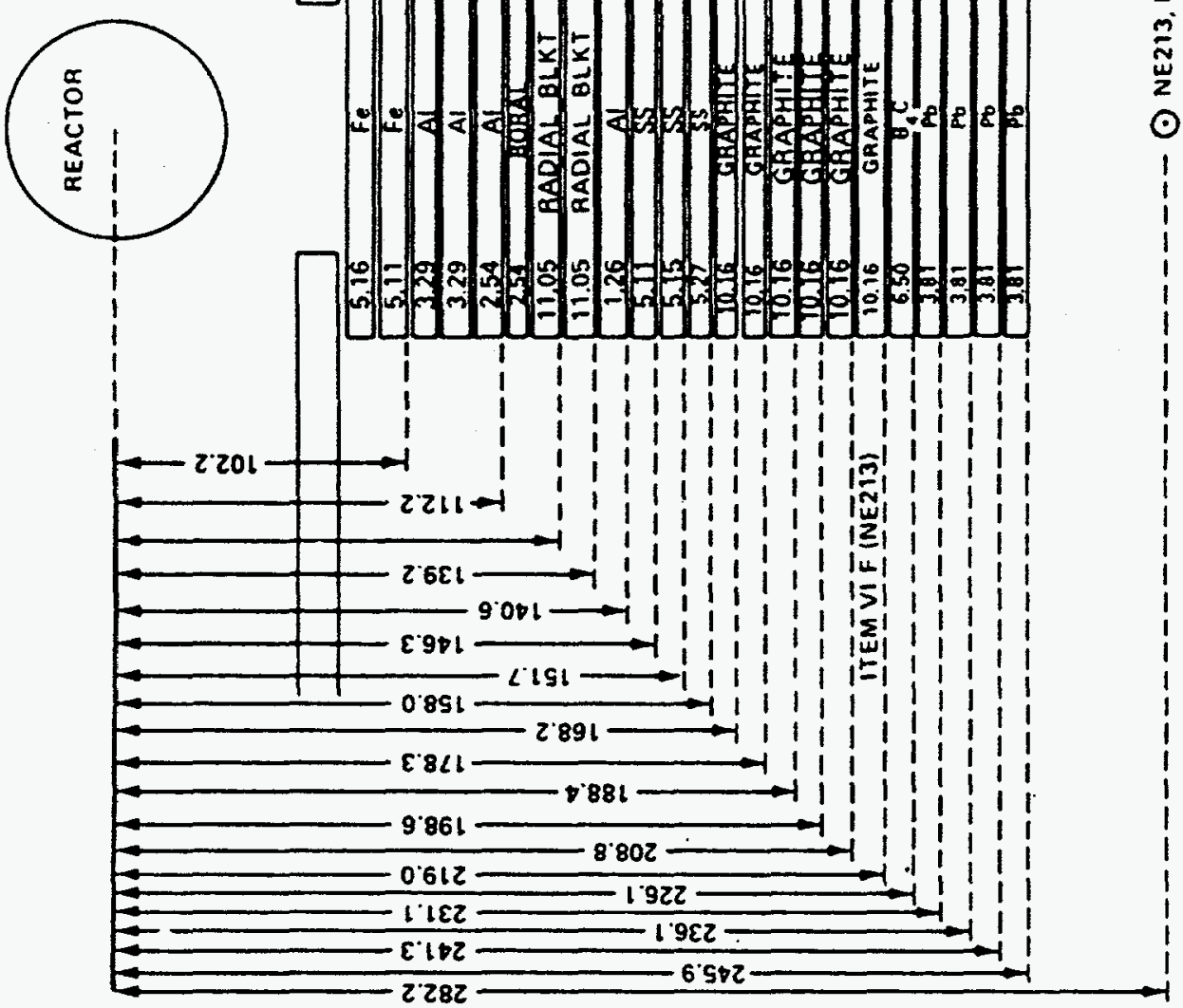

Fig. B22. Sketch of the configuration for measurements under sections VI.F (1-2) of the experimental program plan. 


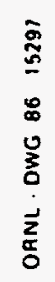

$\begin{array}{ll}m & y \\ \times & 8\end{array}$

$\begin{array}{ll}n & U \\ \times & \mathbf{Z} \\ N & 0\end{array}$

$=\frac{x}{\alpha}$

की

(

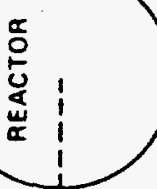

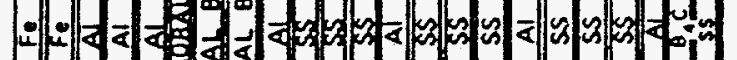

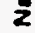
这
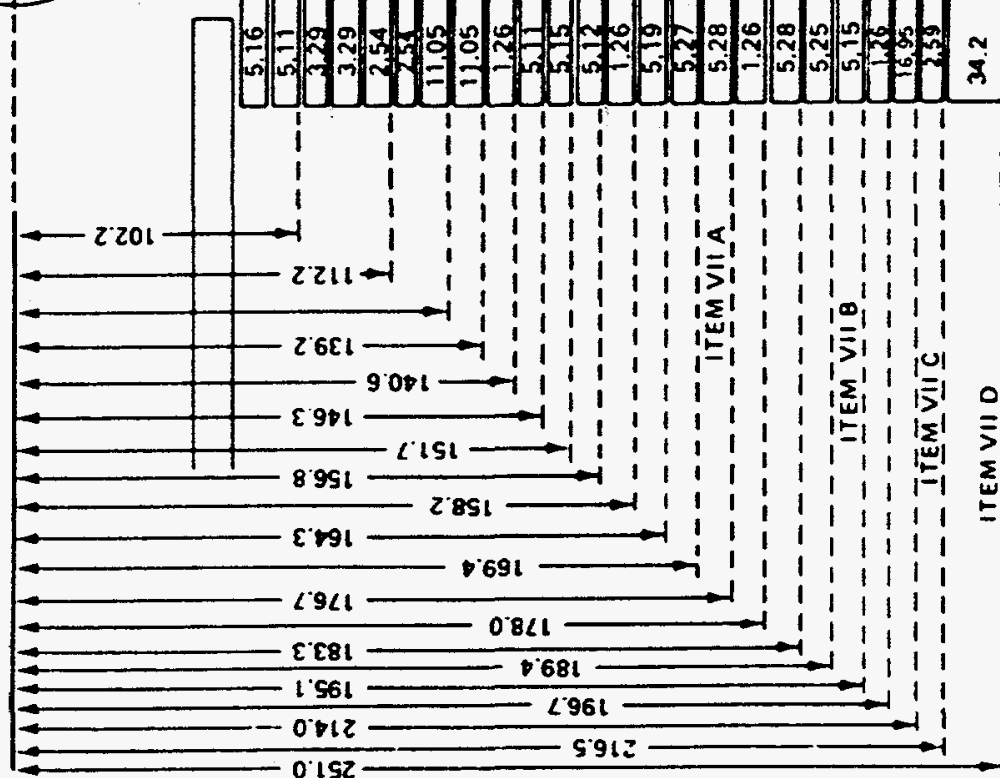

F1gure B23. Sketch of the configuration for measurements under sections VII.A-D of the experimental program plan. 
APPENDIX $C$

ENERGY GROUP STRUCTURES AND MULTIGROUP

DETECTOR RESPONSE FUNCTIONS USED IN CALCULATIONS 
Table C1. 51-Group Response Function for the Bare $\mathrm{BF}_{3}$ Detector. Units are: (Counts $\cdot \mathrm{cm}^{2} /$ neutron).

\begin{tabular}{|c|c|c|c|c|c|}
\hline Group & $\begin{array}{c}\text { Upper } \\
\text { Energy (eV) }\end{array}$ & Response & Group & $\begin{array}{c}\text { Upper } \\
\text { Energy (eV) }\end{array}$ & Response \\
\hline 1 & $1.49200 \mathrm{E}+07^{\mathrm{a}}$ & $6.08293 E-05$ & 27 & $5.24800 E+04$ & $1.28557 \mathrm{E}-03$ \\
\hline 2 & $1.22100 \mathrm{E}+07$ & 9.64387E-05 & 28 & $4.08600 \mathrm{E}+04$ & $1.45530 \mathrm{E}-03$ \\
\hline 3 & $1.00000 \mathrm{E}+07$ & $1.15173 \mathrm{E}-04$ & 29 & $3.18300 \mathrm{E}+04$ & $1.64764 \mathrm{E}-03$ \\
\hline 4 & $8.18700 \mathrm{E}+06$ & $1.38946 \mathrm{E}-04$ & 30 & $2.47900 \mathrm{E}+0.4$ & $1.87795 \mathrm{E}-03$ \\
\hline 5 & $6.70300 E+06$ & $1.82038 \mathrm{E}-04$ & 31 & $1.90300 \mathrm{E}+04$ & $2.11134 E-03$ \\
\hline 6 & $5.48800 \mathrm{E}+06$ & 1.88700 E- 04 & 32 & $1.50300 \mathrm{E}+04$ & $2.71548 E-03$ \\
\hline 7 & $4.49300 \mathrm{E}+06$ & $1.69184 \mathrm{E}-04$ & 33 & $7.10200 E+03$ & $3.67985 \mathrm{E}-03$ \\
\hline 8 & $3.67900 \mathrm{E}+06$ & $1.53397 \mathrm{E}-04$ & 34 & $4.30700 \mathrm{E}+03$ & $4.42658 \mathrm{E}-03$ \\
\hline 9 & $3.01200 E+06$ & $1.66163 \mathrm{E}-04$ & 35 & $3.35500 E+03$ & $5.00872 E-03$ \\
\hline 10 & $2.46600 \mathrm{E}-06$ & $1.94736 \mathrm{E}-04$ & 36 & $2.61300 E+03$ & $5.62130 \mathrm{E}-03$ \\
\hline 11 & $2.01900 E+06$ & $2.41792 \mathrm{E}-04$ & 37 & $2.03500 E+03$ & $6.42654 \mathrm{E}-03$ \\
\hline 12 & $1.65300 \mathrm{E}+06$ & $1.70912 \mathrm{E}-04$ & 38 & $1.58500 \mathrm{E}+03$ & $7.30401 E-03$ \\
\hline 13 & $1.35300 \mathrm{E}+06$ & $1.36139 \mathrm{E}-04$ & 39 & $1.23400 E+03$ & $8.27696 \mathrm{E}-03$ \\
\hline 14 & $1.10800 E+06$ & $1.45402 \mathrm{E}-04$ & 40 & $9.61100 \mathrm{E}+02$ & $1.06452 \mathrm{E}-02$ \\
\hline 15 & $9.07600 \mathrm{E}+05$ & $1.76148 E-04$ & 41 & $4.54000 \mathrm{E}+02$ & $1.55501 \mathrm{E}-02$ \\
\hline 16 & $7.42700 \mathrm{E}+05$ & $2.37209 \mathrm{E}-04$ & 42 & $2.14500 \mathrm{E}+02$ & $2.22467 \mathrm{E}-02$ \\
\hline 17 & $6.08100 \mathrm{E}+05$ & $3.25078 \mathrm{E}-04$ & 43 & $1.01300 \mathrm{E}+02$ & $3.18797 \mathrm{E}-02$ \\
\hline 18 & $4.97800 \mathrm{E}+05$ & 3.84333E-04 & 44 & $4.78500 \mathrm{E}+01$ & $4.73209 \mathrm{E}-02$ \\
\hline 19 & $4.07600 \mathrm{E}+05$ & $3.98219 \mathrm{E}-04$ & 45 & $2.26000 \mathrm{E}+01$ & $7.00329 E-02$ \\
\hline 20 & $3.33700 \mathrm{E}+05$ & 4.81773E-04 & 46 & $1.06800 \mathrm{E}+01$ & $1.01495 \mathrm{E}-01$ \\
\hline 21 & $2.73200 E+05$ & $6.04378 E-04$ & 47 & $5.04300 \mathrm{E}+\infty$ & $1.46607 \mathrm{E}-01$ \\
\hline 22 & $2.23700 \mathrm{E}+05$ & $6.75572 \mathrm{E}-04$ & 48 & $2.38200 E+\infty 0$ & $2.42090 \mathrm{E}-01$ \\
\hline 23 & $1.83200 \mathrm{E}+05$ & $7.35003 \mathrm{E}-04$ & 49 & $1.12500 \mathrm{E}+00$ & $3.58920 \mathrm{E}-01$ \\
\hline 24 & $1.50000 \mathrm{E}+05$ & 7.97585E-04 & 50 & $4.14000 \mathrm{E}-01$ & $5.86870 \mathrm{E}-01$ \\
\hline 25 & $1.22800 \mathrm{E}+05$ & $8.93137 \mathrm{E}-04$ & $51^{b}$ & $1.00000 \mathrm{E}-01$ & $1.11390 \mathrm{E}+\infty$ \\
\hline 26 & $8.65200 E+04$ & $1.08158 \mathrm{E}-03$ & & & \\
\hline
\end{tabular}

${ }^{2}$ Read as $1.49200 \times 10^{7}$.

b Lower energy boundary is $1.0 \times 10^{-5} \mathrm{eV}$. 
Table C2. 51-Group Response Function for the Cd-Covered $\mathrm{BF}_{3}$ Detector. Units are: (Counts $\cdot \mathrm{cm}^{2} /$ neutron).

\begin{tabular}{|c|c|c|c|c|c|}
\hline Group & $\begin{array}{c}\text { Upper } \\
\text { Energy (eV) }\end{array}$ & Response & Group & $\begin{array}{c}\text { Upper } \\
\text { Energy (eV) }\end{array}$ & Response \\
\hline 1 & $1.49200 \mathrm{E}+07^{2}$ & $6.08293 \mathrm{E}-05$ & 27 & $5.24800 \mathrm{E}+04$ & $1.28557 \mathrm{E}-03$ \\
\hline 2 & $1.22100 \mathrm{E}+07$ & $9.64387 \mathrm{E}-05$ & 28 & $4.08600 \mathrm{E}+04$ & $1.45530 \mathrm{E}-03$ \\
\hline 3 & $1.00000 E+07$ & 1.15173E-04 & 29 & $3.18300 \mathrm{E}+04$ & $1.64764 \mathrm{E}-03$ \\
\hline 4 & $8.18700 \mathrm{E}+06$ & $1.38946 \mathrm{E}-04$ & 30 & $2.47900 E+04$ & $1.87795 \mathrm{E}-03$ \\
\hline 5 & $6.70300 E+06$ & $1.82038 E-04$ & 31 & $1.90300 \mathrm{E}+04$ & $2.11134 \mathrm{E}-03$ \\
\hline 6 & $5.48800 \mathrm{E}+06$ & $1.88700 \mathrm{E}-04$ & 32 & $1.50300 \mathrm{E}+04$ & 2.71548E-03 \\
\hline 7 & $4.49300 \mathrm{E}+06$ & $1.69184 \mathrm{E}-04$ & 33 & $7.10200 \mathrm{E}+03$ & $3.67985 \mathrm{E}-03$ \\
\hline 8 & $3.67900 \mathrm{E}+06$ & $1.53397 \mathrm{E}-04$ & 34 & $4.30700 E+03$ & $4.42658 \mathrm{E}-03$ \\
\hline 9 & $3.01200 E+06$ & $1.66163 \mathrm{E}-04$ & 35 & $3.35500 \mathrm{E}+03$ & $5.00872 \mathrm{E}-03$ \\
\hline 10 & $2.46600 \mathrm{E}-06$ & $1.94736 \mathrm{E}-04$ & 36 & $2.61300 \mathrm{E}+03$ & $5.62130 \mathrm{E}-03$ \\
\hline 11 & $2.01900 \mathrm{E}+06$ & $2.41792 \mathrm{E}-04$ & 37 & $2.03500 E+03$ & $6.42654 \mathrm{E}-03$ \\
\hline 12 & $1.65300 \mathrm{E}+06$ & $1.70912 \mathrm{E}-04$ & 38 & $1.58500 \mathrm{E}+03$ & $7.30401 \mathrm{E}-03$ \\
\hline 13 & $1.35300 \mathrm{E}+06$ & $1.36139 \mathrm{E}-04$ & 39 & $1.23400 \mathrm{E}+03$ & 8.27696E-03 \\
\hline 14 & $1.10800 \mathrm{E}+06$ & $1.45402 E-04$ & 40 & $9.61100 \mathrm{E}+02$ & $1.06452 \mathrm{E}-02$ \\
\hline 15 & $9.07600 \mathrm{E}+05$ & $1.76148 \mathrm{E}-04$ & 41 & $4.54000 \mathrm{E}+02$ & $1.55501 \mathrm{E}-02$ \\
\hline 16 & $7.42700 \mathrm{E}+05$ & $2.37209 \mathrm{E}-04$ & 42 & $2.14500 \mathrm{E}+02$ & $2.22467 \mathrm{E}-02$ \\
\hline 17 & $6.08100 E+05$ & $3.25078 \mathrm{E}-04$ & 43 & $1.01300 \mathrm{E}+02$ & 3.18797E-02 \\
\hline 18 & $4.97800 \mathrm{E}+05$ & $3.84333 \mathrm{E}-04$ & 44 & $4.78500 \mathrm{E}+01$ & $4.73209 \mathrm{E}-02$ \\
\hline 19 & $4.07600 E+05$ & $3.98219 \mathrm{E}-04$ & 45 & $2.26000 E+01$ & $7.00329 E-02$ \\
\hline 20 & $3.33700 \mathrm{E}+05$ & $4.81773 E-04$ & 46 & $1.06800 E+01$ & $1.01495 \mathrm{E}-01$ \\
\hline 21 & $2.73200 E+05$ & $6.04378 \mathrm{E}-04$ & 47 & $5.04300 \mathrm{E}+00$ & $1.46607 \mathrm{E}-01$ \\
\hline 22 & $2.23700 \mathrm{E}+05$ & $6.75572 \mathrm{E}-04$ & 48 & $2.38200 E+00$ & $2.07692 \mathrm{E}-01$ \\
\hline 23 & $1.83200 E+05$ & 7.35003E-04 & 49 & $1.12500 \mathrm{E}+\infty 0$ & $2.28613 \mathrm{E}-01$ \\
\hline 24 & $1.50000 E+05$ & $7.97585 \mathrm{E}-04$ & 50 & $4.14000 \mathrm{E}-01$ & 0.0 \\
\hline 25 & $1.22800 \mathrm{E}+05$ & $8.93137 \mathrm{E}-04$ & $51^{b}$ & $1.00000 \mathrm{E}-01$ & 0.0 \\
\hline 26 & $8.65200 \mathrm{E}+04$ & $1.08158 \mathrm{E}-03$ & & & \\
\hline
\end{tabular}

${ }^{2}$ Read as $1.49200 \times 10^{7}$.

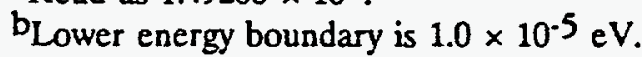


Table C3. 51-Group Response Function for the 3-inch Bonner Ball. Units are: (Counts $\cdot \mathrm{cm}^{2} /$ neutron).

\begin{tabular}{|c|c|c|c|c|c|}
\hline Group & $\begin{array}{c}\text { Upper } \\
\text { Energy (eV) }\end{array}$ & Response & Group & $\begin{array}{c}\text { Upper } \\
\text { Energy }(\mathrm{eV})\end{array}$ & Response \\
\hline 1 & $1.49200 \mathrm{E}+07^{2}$ & $3.44697 \mathrm{E}-03$ & 27 & $5.24800 \mathrm{E}+04$ & $2.60266 \mathrm{E}-01$ \\
\hline 2 & $1.22100 \mathrm{E}+07$ & $4.36754 \mathrm{E}-03$ & 28 & $4.08600 \mathrm{E}+04$ & $2.76680 \mathrm{E}-01$ \\
\hline 3 & $1.00000 \mathrm{E}+07$ & $5.50543 \mathrm{E}-03$ & 29 & $3.18300 \mathrm{E}+04$ & $2.92975 \mathrm{E}-01$ \\
\hline 4 & $8.18700 E+06$ & $6.86545 \mathrm{E}-03$ & 30 & $2.47900 \mathrm{E}+04$ & $3.10526 \mathrm{E}-01$ \\
\hline 5 & $6.70300 \mathrm{E}+06$ & $9.24691 \mathrm{E}-03$ & 31 & $1.90300 \mathrm{E}+04$ & $3.26311 \mathrm{E}-01$ \\
\hline 6 & $5.48800 \mathrm{E}+06$ & $1.10365 \mathrm{E}-02$ & 32 & $1.50300 \mathrm{E}+04$ & $3.61419 \mathrm{E}-01$ \\
\hline 7 & $4.49300 \mathrm{E}+06$ & $1.34925 \mathrm{E}-02$ & 33 & $7.10200 \mathrm{E}+03$ & $4.07614 \mathrm{E}-01$ \\
\hline 8 & $3.67900 \mathrm{E}+06$ & $1.69481 \mathrm{E}-02$ & 34 & $4.30700 E+03$ & 4.37120E-01 \\
\hline 9 & $3.01200 \mathrm{E}+06$ & $2.11136 \mathrm{E}-02$ & 35 & $3.35500 \mathrm{E}+03$ & $4.58520 \mathrm{E}-01$ \\
\hline 10 & $2.46600 \mathrm{E}-06$ & $2.61091 \mathrm{E}-02$ & 36 & $2.61300 E+03$ & $4.81026 \mathrm{E}-01$ \\
\hline 11 & $2.01900 \mathrm{E}+06$ & $3.19911 \mathrm{E}-02$ & 37 & $2.03500 \mathrm{E}+03$ & 5.04895E-01 \\
\hline 12 & $1.65300 \mathrm{E}+06$ & $3.88334 \mathrm{E}-02$ & 38 & $1.58500 \mathrm{E}+03$ & 5.30163E-01 \\
\hline 13 & $1.35300 E+06$ & 4.67591E-02 & 39 & $1.23400 \mathrm{E}+03$ & $5.55102 E-01$ \\
\hline 14 & $1.10800 \mathrm{E}+06$ & $5.58187 \mathrm{E}-02$ & 40 & $9.61100 E+02$ & $6.07197 \mathrm{E}-01$ \\
\hline 15 & $9.07600 \mathrm{E}+05$ & $6.59898 \mathrm{E}-02$ & 41 & $4.54000 \mathrm{E}+02$ & $6.98568 \mathrm{E}-01$ \\
\hline 16 & $7.42700 \mathrm{E}+05$ & $7.72060 \mathrm{E}-02$ & 42 & $2.14500 \mathrm{E}+02$ & $7.76309 \mathrm{E}-01$ \\
\hline 17 & $6.08100 \mathrm{E}+05$ & $8.94341 \mathrm{E}-02$ & 43 & $1.01300 \mathrm{E}+02$ & $7.87411 E-01$ \\
\hline 18 & $4.97800 \mathrm{E}+05$ & $1.02508 \mathrm{E}-01$ & 44 & $4.78500 \mathrm{E}+01$ & 8.18827E-01 \\
\hline 19 & $4.07600 \mathrm{E}+05$ & $1.16211 \mathrm{E}-01$ & 45 & $2.26000 \mathrm{E}+01$ & $9.40470 \mathrm{E}-01$ \\
\hline 20 & $3.33700 \mathrm{E}+05$ & $1.30279 \mathrm{E}-01$ & 46 & $1.06800 \mathrm{E}+01$ & $1.04548 \mathrm{E}-00$ \\
\hline 21 & $2.73200 \mathrm{E}+05$ & $1.44609 \mathrm{E}-01$ & 47 & $5.04300 E+\infty 0$ & $1.13841 E-\infty 0$ \\
\hline 22 & $2.23700 \mathrm{E}+05$ & $1.59106 \mathrm{E}-01$ & 48 & $2.38200 \mathrm{E}+\infty 0$ & $1.18444 \mathrm{E}-00$ \\
\hline 23 & $1.83200 \mathrm{E}+05$ & $1.73572 \mathrm{E}-01$ & 49 & $1.12500 \mathrm{E}+\infty 0$ & $8.66930 \mathrm{E}-01$ \\
\hline 24 & $1.50000 \mathrm{E}+05$ & $1.87950 \mathrm{E}-01$ & 50 & $4.14000 \mathrm{E}-01$ & 0.0 \\
\hline 25 & $1.22800 \mathrm{E}+05$ & $2.07008 \mathrm{E}-01$ & $51^{b}$ & $1.00000 \mathrm{E}-01$ & 0.0 \\
\hline 26 & $8.65200 E+04$ & 2.35597E-01 & & & \\
\hline
\end{tabular}

${ }^{2}$ Read as $1.49200 \times 10^{7}$.

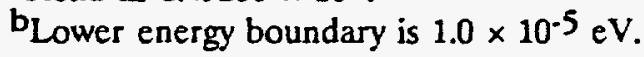


Table C4. 51-Group Response Function for the 5-inch Bonner Ball. Units are: (Counts $\cdot \mathrm{cm}^{2} /$ neutron).

\begin{tabular}{|c|c|c|c|c|c|}
\hline Group & $\begin{array}{c}\text { Upper } \\
\text { Energy (eV) }\end{array}$ & Response & Group & $\begin{array}{c}\text { Upper } \\
\text { Energy (eV) }\end{array}$ & Response \\
\hline 1 & $1.49200 \mathrm{E}+07^{\mathrm{a}}$ & $1.20669 \mathrm{E}-01$ & 27 & $5.24800 \mathrm{E}+04$ & $1.23880 \mathrm{E}+00$ \\
\hline 2 & $1.22100 \mathrm{E}+07$ & $1.55463 \mathrm{E}-01$ & 28 & $4.08600 \mathrm{E}+04$ & $1.23960 \mathrm{E}+00$ \\
\hline 3 & $1.00000 \mathrm{E}+07$ & $1.98617 \mathrm{E}-01$ & 29 & $3.18300 \mathrm{E}+04$ & $1.24050 \mathrm{E}+00$ \\
\hline 4 & $8.18700 E+06$ & $2.47844 \mathrm{E}-01$ & 30 & $2.47900 \mathrm{E}+04$ & $1.24352 \mathrm{E}+00$ \\
\hline 5 & $6.70300 E+06$ & $3.09165 \mathrm{E}-01$ & 31 & $1.90300 \mathrm{E}+04$ & $1.24570 \mathrm{E}+00$ \\
\hline 6 & $5.48800 E+06$ & $3.50830 \mathrm{E}-01$ & 32 & $1.50300 \mathrm{E}+04$ & $1.25470 \mathrm{E}+00$ \\
\hline 7 & $4.49300 E+06$ & $4.11506 \mathrm{E}-01$ & 33 & $7.10200 E+03$ & $1.26700 \mathrm{E}+00$ \\
\hline 8 & $3.67900 E+06$ & 4.80377E-01 & 34 & $4.30700 E+03$ & $1.27370 \mathrm{E}+00$ \\
\hline 9 & $3.01200 E+06$ & $5.59543 \mathrm{E}-01$ & 35 & $3.35500 \mathrm{E}+03$ & $1.27970 \mathrm{E}+00$ \\
\hline 10 & $2.46600 \mathrm{E}-06$ & $6.44104 \mathrm{E}-01$ & 36 & $2.61300 E+03$ & $1.28640 E+00$ \\
\hline 11 & $2.01900 \mathrm{E}+06$ & 7.27624E-01 & 37 & $2.03500 E+03$ & $1.29219 \mathrm{E}+00$ \\
\hline 12 & $1.65300 E+06$ & 8.07973E-01 & 38 & $1.58500 E+03$ & $1.29859 \mathrm{E}+00$ \\
\hline 13 & $1.35300 \mathrm{E}+06$ & $8.84155 \mathrm{E}-01$ & 39 & $1.23400 \mathrm{E}+03$ & $1.30090 \mathrm{E}+00$ \\
\hline 14 & $1.10800 \mathrm{E}+06$ & $9.54882 \mathrm{E}-01$ & 40 & $9.61100 \mathrm{E}+02$ & $1.29933 \mathrm{E}+00$ \\
\hline 15 & $9.07600 E+05$ & $1.01825 E+00$ & 41 & $4.54000 \mathrm{E}+02$ & $1.30253 \mathrm{E}+00$ \\
\hline 16 & $7.42700 E+05$ & $1.07320 E+00$ & 42 & $2.14500 \mathrm{E}+02$ & $1.25907 \mathrm{E}+00$ \\
\hline 17 & $6.08100 \mathrm{E}+05$ & $1.12007 E+00$ & 43 & $1.01300 \mathrm{E}+02$ & $1.22880 E+00$ \\
\hline 18 & $4.97800 E+05$ & $1.15811 E+\infty 0$ & 44 & $4.78500 \mathrm{E}+01$ & $1.23420 \mathrm{E}+00$ \\
\hline 19 & $4.07600 E+05$ & $1.18736 \mathrm{E}+\infty 0$ & 45 & $2.26000 \mathrm{E}+01$ & $1.21014 \mathrm{E}+00$ \\
\hline 20 & $3.33700 E+05$ & $1.20946 \mathrm{E}+00$ & 46 & $1.06800 \mathrm{E}+01$ & $1.14298 E+00$ \\
\hline 21 & $2.73200 \mathrm{E}+05$ & $1.22430 \mathrm{E}+00$ & 47 & $5.04300 \mathrm{E}+00$ & $1.05081 E+00$ \\
\hline 22 & $2.23700 \mathrm{E}+05$ & $1.23395 \mathrm{E}+00$ & 48 & $2.38200 \mathrm{E}+00$ & $9.13858 \mathrm{E}-01$ \\
\hline 23 & $1.83200 \mathrm{E}+05$ & $1.23969 \mathrm{E}+00$ & 49 & $1.12500 E+00$ & $5.47583 \mathrm{E}-01$ \\
\hline 24 & $1.50000 \mathrm{E}+05$ & $1.24235 \mathrm{E}+00$ & 50 & $4.14000 \mathrm{E}-01$ & 0.0 \\
\hline 25 & $1.22800 \mathrm{E}+05$ & $1.24263 E+00$ & $51^{\mathrm{b}}$ & $1.00000 \mathrm{E}-01$ & 0.0 \\
\hline 26 & $8.65200 E+04$ & $1.24045 \mathrm{E}+00$ & & & \\
\hline
\end{tabular}

${ }^{a}$ Read as $1.49200 \times 10^{7}$.

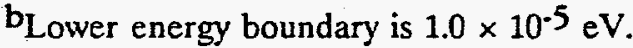


Table C5. 51-Group Response Function for the 8-inch Bonner Ball.

Units are: (Counts $\cdot \mathrm{cm}^{2} /$ ncutron).

\begin{tabular}{|c|c|c|c|c|c|}
\hline Group & $\begin{array}{c}\text { Upper } \\
\text { Energy (eV) }\end{array}$ & Response & Group & $\begin{array}{c}\text { Upper } \\
\text { Energy }(\mathrm{eV})\end{array}$ & Response \\
\hline 1 & $1.49200 \mathrm{E}+07^{2}$ & 4.32326E-01 & 27 & $5.24800 \mathrm{E}+04$ & $6.78782 E-01$ \\
\hline 2 & $1.22100 \mathrm{E}+07$ & $5.39892 \mathrm{E}-01$ & 28 & $4.08600 E+04$ & $6.55690 \mathrm{E}-01$ \\
\hline 3 & $1.00000 E+07$ & $6.67276 \mathrm{E}-01$ & 29 & $3.18300 \mathrm{E}+04$ & $6.35525 \mathrm{E}-01$ \\
\hline 4 & $8.18700 E+06$ & 7.86134E-01 & 30 & $2.47900 E+04$ & $6.17751 E-01$ \\
\hline 5 & $6.70300 E+06$ & $9.07779 \mathrm{E}-01$ & 31 & $1.90300 \mathrm{E}+04$ & $6.02962 E-01$ \\
\hline 6 & $5.48800 E+06$ & $9.90159 \mathrm{E}-01$ & 32 & $1.50300 \mathrm{E}+04$ & $5.78139 E-01$ \\
\hline 7 & $4.49300 \mathrm{E}+06$ & $1.06950 E+\infty$ & 33 & $7.10200 E+03$ & $5.51763 \mathrm{E}-01$ \\
\hline 8 & $3.67900 \mathrm{E}+06$ & $1.14892 E+\infty$ & 34 & $4.30700 E+03$ & $5.37200 \mathrm{E}-01$ \\
\hline 9 & $3.01200 E+06$ & $1.24834 \mathrm{E}+\infty 0$ & 35 & $3.35500 E+03$ & $5.28644 E-01$ \\
\hline 10 & $2.46600 \mathrm{E}-06$ & $1.33619 E+00$ & 36 & $2.61300 E+03$ & $5.20655 \mathrm{E}-01$ \\
\hline 11 & $2.01900 E+06$ & $1.39025 \mathrm{E}+\infty 0$ & 37 & $2.03500 E+03$ & $5.12425 \mathrm{E}-01$ \\
\hline 12 & $1.65300 \mathrm{E}+06$ & $1.41070 E+\infty 0$ & 38 & $1.58500 \mathrm{E}+03$ & $5.04734 \mathrm{E}-01$ \\
\hline 13 & $1.35300 \mathrm{E}+06$ & $1.40779 E+00$ & 39 & $1.23400 \mathrm{E}+03$ & 4.95599E-01 \\
\hline 14 & $1.10800 \mathrm{E}+06$ & $1.38561 \mathrm{E}+00$ & 40 & $9.61100 \mathrm{E}+02$ & 4.75849E-01 \\
\hline 15 & $9.07600 \mathrm{E}+05$ & $1.34711 \mathrm{E}+\infty 0$ & 41 & $4.54000 E+02$ & 4.50639E-01 \\
\hline 16 & $7.42700 E+05$ & $1.29614 E+\infty 0$ & 42 & $2.14500 \mathrm{E}+02$ & 4.12817E-01 \\
\hline 17 & $6.08100 \mathrm{E}+05$ & $1.23801 E+\infty$ & 43 & $1.01300 \mathrm{E}+02$ & 4.01933E-01 \\
\hline 18 & $4.97800 \mathrm{E}+05$ & $1.17527 \mathrm{E}+00$ & 44 & $4.78500 \mathrm{E}+01$ & 3.85773E-01 \\
\hline 19 & $4.07600 \mathrm{E}+05$ & $1.11077 \mathrm{E}+\infty 0$ & 45 & $2.26000 \mathrm{E}+01$ & 3.62755E-01 \\
\hline 20 & $3.33700 \mathrm{E}+05$ & $1.04936 \mathrm{E}+00$ & 46 & $1.06800 \mathrm{E}+01$ & $3.30363 E-01$ \\
\hline 21 & $2.73200 \mathrm{E}+05$ & 9.89847E-01 & 47 & $5.04300 \mathrm{E}+00$ & $2.94706 \mathrm{E}-01$ \\
\hline 22 & $2.23700 \mathrm{E}+05$ & $9.34578 \mathrm{E}-01$ & 48 & $2.38200 E+00$ & 2.50431E-01 \\
\hline 23 & $1.83200 \mathrm{E}+05$ & 8.84631E-01 & 49 & $1.12500 \mathrm{E}+00$ & $1.47241 E-01$ \\
\hline 24 & $1.50000 E+05$ & $8.39704 E-01$ & 50 & $4.14000 \mathrm{E}-01$ & 0.0 \\
\hline 25 & $1.22800 \mathrm{E}+05$ & $7.87495 \mathrm{E}-01$ & $51^{b}$ & $1.00000 \mathrm{E}-01$ & 0.0 \\
\hline 26 & $8.65200 E+04$ & 7.23162E-01 & & & \\
\hline
\end{tabular}

${ }^{2}$ Read as $1.49200 \times 10^{7}$.

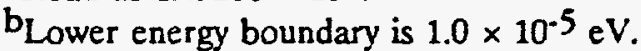


Table C6. 51-Group Response Function for the 10-inch Bonner Ball. Units are: (Counts $\cdot \mathrm{cm}^{2} /$ neutron).

\begin{tabular}{|c|c|c|c|c|c|}
\hline Group & $\begin{array}{c}\text { Upper } \\
\text { Energy (eV) }\end{array}$ & Response & Group & $\begin{array}{c}\text { Upper } \\
\text { Energy (eV) }\end{array}$ & Response \\
\hline 1 & $1.49200 \mathrm{E}+07^{2}$ & $5.78909 E-01$ & 27 & $5.24800 \mathrm{E}+04$ & $2.69356 \mathrm{E}-01$ \\
\hline 2 & $1.22100 \mathrm{E}+07$ & $7.00739 \mathrm{E}-01$ & 28 & $4.08600 \mathrm{E}+04$ & $2.57060 \mathrm{E}-01$ \\
\hline 3 & $1.00000 E+07$ & $8.39563 \mathrm{E}-01$ & 29 & $3.18300 \mathrm{E}+04$ & 2.46583E-01 \\
\hline 4 & $8.18700 E+06$ & $9.46638 \mathrm{E}-01$ & 30 & $2.47900 \mathrm{E}+04$ & 2.37427E-01 \\
\hline 5 & $6.70300 \mathrm{E}+06$ & $1.04566 E+\infty 0$ & 31 & $1.90300 \mathrm{E}+04$ & 2.29981E-01 \\
\hline 6 & $5.48800 \mathrm{E}+06$ & $1.10766 \mathrm{E}+\infty$ & 32 & $1.50300 E+04$ & $2.17578 \mathrm{E}-01$ \\
\hline 7 & $4.49300 \mathrm{E}+06$ & $1.12520 \mathrm{E}+\infty$ & 33 & $7.10200 \mathrm{E}+03$ & $2.04694 E-01$ \\
\hline 8 & $3.67900 \mathrm{E}+06$ & $1.14494 E+\infty$ & 34 & $4.30700 E+03$ & $1.97790 \mathrm{E}-01$ \\
\hline 9 & $3.01200 \mathrm{E}+06$ & $1.18825 E+00$ & 35 & $3.35500 \mathrm{E}+03$ & $1.93732 \mathrm{E}-01$ \\
\hline 10 & $2.46600 \mathrm{E}-06$ & $1.21060 \mathrm{E}+\infty 0$ & 36 & $2.61300 E+03$ & $1.89962 \mathrm{E}-01$ \\
\hline 11 & $2.01900 E+06$ & $1.18985 E+00$ & 37 & $2.03500 \mathrm{E}+03$ & $1.86172 \mathrm{E}-01$ \\
\hline 12 & $1.65300 \mathrm{E}+06$ & $1.13338 \mathrm{E}+\infty$ & 38 & $1.58500 \mathrm{E}+03$ & $1.82642 \mathrm{E}-01$ \\
\hline 13 & $1.35300 E+06$ & $1.06001 \mathrm{E}+00$ & 39 & $1.23400 E+03$ & $1.78640 \mathrm{E}-01$ \\
\hline 14 & $1.10800 E+06$ & $9.77245 \mathrm{E}-01$ & 40 & $9.61100 \mathrm{E}+02$ & $1.70306 \mathrm{E}-01$ \\
\hline 15 & $9.07600 \mathrm{E}+05$ & $8.90321 E-01$ & 41 & $4.54000 E+02$ & $1.59811 E-01$ \\
\hline 16 & $7.42700 E+05$ & $8.03739 \mathrm{E}-01$ & 42 & $2.14500 E+02$ & $1.45263 \mathrm{E}-01$ \\
\hline 17 & $6.08100 \mathrm{E}+05$ & $7.22208 \mathrm{E}-01$ & 43 & $1.01300 \mathrm{E}+02$ & $1.53973 E-01$ \\
\hline 18 & $4.97800 \mathrm{E}+05$ & $6.47158 \mathrm{E}-01$ & 44 & $4.78500 \mathrm{E}+01$ & $1.47152 \mathrm{E}-01$ \\
\hline 19 & $4.07600 \mathrm{E}+05$ & $5.79948 \mathrm{E}-01$ & 45 & $2.26000 \mathrm{E}+01$ & $1.37947 \mathrm{E}-01$ \\
\hline 20 & $3.33700 \mathrm{E}+05$ & $5.22467 \mathrm{E}-01$ & 46 & $1.06800 \mathrm{E}+01$ & $1.25359 \mathrm{E}-01$ \\
\hline 21 & $2.73200 \mathrm{E}+05$ & $4.72366 \mathrm{E}-01$ & 47 & $5.04300 \mathrm{E}+00$ & $1.11702 \mathrm{E}-01$ \\
\hline 22 & $2.23700 \mathrm{E}+05$ & 4.29680E-01 & 48 & $2.38200 \mathrm{E}+\infty$ & $9.48828 \mathrm{E}-02$ \\
\hline 23 & $1.83200 \mathrm{E}+05$ & $3.93933 \mathrm{E}-01$ & 49 & $1.12500 \mathrm{E}+\infty 0$ & $5.57513 E-02$ \\
\hline 24 & $1.50000 E+05$ & $3.63864 \mathrm{E}-01$ & 50 & $4.14000 \mathrm{E}-01$ & 0.0 \\
\hline 25 & $1.22800 \mathrm{E}+05$ & $3.31235 \mathrm{E}-01$ & $51^{b}$ & $1.00000 \mathrm{E}-01$ & 0.0 \\
\hline 26 & $8.65200 \mathrm{E}+04$ & $2.93774 \mathrm{E}-01$ & & & \\
\hline
\end{tabular}

${ }^{\text {a Read as }} 1.49200 \times 10^{7}$.

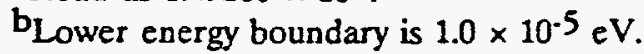


Table C7. 61-Group Response Function for the Bare $\mathrm{BF}_{3}$ Detector.

Units are: (Counts $\cdot \mathrm{cm}^{2} /$ neutron).

\begin{tabular}{|c|c|c|c|c|c|}
\hline Group & $\begin{array}{c}\text { Upper } \\
\text { Energy (eV) }\end{array}$ & Response & Group & $\begin{array}{c}\text { Upper } \\
\text { Encrgy (eV) }\end{array}$ & Response \\
\hline 1 & $1.49183 E+07^{2}$ & $6.08293 \mathrm{E}-05$ & 32 & $8.65168 E+04$ & $1.08158 \mathrm{E}-03$ \\
\hline 2 & $1.22140 \mathrm{E}+07$ & $9.63804 \mathrm{E}-05$ & 33 & $5.65622 \mathrm{E}+04$ & $1.08183 \mathrm{E}-03$ \\
\hline 3 & $1.00000 E+07$ & 1.15173E-04 & 34 & $5.24752 E+04$ & $1.35538 \mathrm{E}-03$ \\
\hline 4 & $8.18730 \mathrm{E}+06$ & $1.38942 E-04$ & 35 & $3.43067 \mathrm{E}+04$ & $1.56990 \mathrm{E}-03$ \\
\hline 5 & $6.70320 \mathrm{E}+06$ & $1.82032 \mathrm{E}-04$ & 36 & $2.85011 E+04$ & $1.64764 \mathrm{E}-03$ \\
\hline 6 & $5.48811 E+06$ & $1.88699 \mathrm{E}-04$ & 37 & $2.70001 E+04$ & $1.64764 \mathrm{E}-03$ \\
\hline 7 & $4.49329 E+06$ & $1.69186 \mathrm{E}-04$ & 38 & $2.60584 \mathrm{E}+04$ & $1.64810 \mathrm{E}-03$ \\
\hline 8 & $3.67879 E+06$ & $1.53398 \mathrm{E}-04$ & 39 & $2.47875 E+04$ & $1.87795 \mathrm{E}-03$ \\
\hline 9 & $3.01194 \mathrm{E}+06$ & $1.66165 \mathrm{E}-04$ & 40 & $2.35786 \mathrm{E}+04$ & $2.00018 E-03$ \\
\hline 10 & $2.46597 \mathrm{E}+06$ & $1.94736 \mathrm{E}-04$ & 41 & $1.50344 E+04$ & $2.71513 \mathrm{E}-03$ \\
\hline 11 & $2.34570 \mathrm{E}+06$ & $1.94736 \mathrm{E}-04$ & 42 & $9.11882 E+03$ & 3.19773E-03 \\
\hline 12 & $2.23130 \mathrm{E}+06$ & $1.94743 \mathrm{E}-04$ & 43 & $5.53084 E+03$ & $3.95969 \mathrm{E}-03$ \\
\hline 13 & $2.01897 \mathrm{E}+06$ & $2.41790 \mathrm{E}-04$ & 44 & $3.70744 E+03$ & 4.71797E-03 \\
\hline 14 & $1.65299 E+06$ & $1.70912 \mathrm{E}-04$ & 45 & $3.03539 E+03$ & $5.00935 E-03$ \\
\hline 15 & $1.35335 E+06$ & $1.36184 \mathrm{E}-04$ & 46 & $2.61259 E+03$ & $5.62130 \mathrm{E}-03$ \\
\hline 16 & $1.10803 E+06$ & $1.45472 \mathrm{E}-04$ & 47 & $2.24867 \mathrm{E}+03$ & $5.62257 \mathrm{E}-03$ \\
\hline 17 & $9.07180 E+05$ & $1.76148 \mathrm{E}-04$ & 48 & $2.03468 \mathrm{E}+03$ & $6.86570 \mathrm{E}-03$ \\
\hline 18 & $7.42735 E+05$ & 2.37194E-04 & 49 & $1.23410 \mathrm{E}+03$ & $9.46083 \mathrm{E}-03$ \\
\hline 19 & $6.08101 E+05$ & $3.25077 \mathrm{E}-04$ & 50 & $7.48518 E+02$ & $1.06452 \mathrm{E}-02$ \\
\hline 20 & $5.23397 \mathrm{E}+05$ & $3.25078 \mathrm{E}-04$ & 51 & $4.53999 E+02$ & $1.55501 \mathrm{E}-02$ \\
\hline 21 & $4.97870 E+05$ & $3.87073 \mathrm{E}-04$ & 52 & $2.75364 E+02$ & $1.89013 \mathrm{E}-02$ \\
\hline 22 & $3.87742 E+05$ & 4.31607E-04 & 53 & $1.67017 \mathrm{E}+02$ & $2.22467 \mathrm{E}-02$ \\
\hline 23 & $3.01974 E+05$ & $4.81773 \mathrm{E}-04$ & 54 & $1.01301 E+02$ & $3.18796 \mathrm{E}-02$ \\
\hline 24 & $2.98491 E+05$ & 4.81773E-04 & 55 & $4.78512 \mathrm{E}+01$ & 4.73204E-02 \\
\hline 25 & $2.97211 \mathrm{E}+05$ & $4.81773 E-04$ & 56 & $2.26033 E+01$ & $7.00402 \mathrm{E}-02$ \\
\hline 26 & $2.94518 E+05$ & 4.81773E-04 & 57 & $1.06770 \mathrm{E}+01$ & $1.01495 \mathrm{E}-01$ \\
\hline 27 & $2.73237 E+05$ & $6.04295 \mathrm{E}-04$ & 58 & $5.04348 E+00$ & $1.46601 \mathrm{E}-01$ \\
\hline 28 & $2.23708 E+05$ & $6.75630 \mathrm{E}-04$ & 59 & $2.38237 E+\infty$ & $2.42070 \mathrm{E}-01$ \\
\hline 29 & $1.83156 \mathrm{E}+05$ & 7.35095E-04 & 60 & $1.12535 E+00$ & $3.58887 \mathrm{E}-01$ \\
\hline 30 & $1.49956 \mathrm{E}+05$ & $7.97690 \mathrm{E}-04$ & $61^{b}$ & $4.13994 \mathrm{E}-01$ & $1.04347 E+\infty$ \\
\hline 31 & $1.22773 E+05$ & $8.93156 \mathrm{E}-04$ & & & \\
\hline
\end{tabular}

${ }^{2}$ Read as $1.49183 \times 10^{7}$.

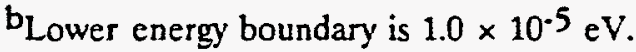


Table C8. 61-Group Response Function for the Cd-Covered $\mathrm{BF}_{3}$ Detector. Units are: (Counts $\cdot \mathrm{cm}^{2} /$ ncutron).

\begin{tabular}{|c|c|c|c|c|c|}
\hline Group & $\begin{array}{c}\text { Upper } \\
\text { Energy (eV) }\end{array}$ & Response & Group & $\begin{array}{c}\text { Upper } \\
\text { Energy (eV) }\end{array}$ & Response \\
\hline 1 & $1.49183 E+07^{2}$ & $6.08293 \mathrm{E}-05$ & 32 & $8.65168 E+04$ & $1.08158 \mathrm{E}-03$ \\
\hline 2 & $1.22140 E+07$ & 9.63804E-05 & 33 & $5.65622 \mathrm{E}+04$ & $1.08183 E-03$ \\
\hline 3 & $1.00000 E+07$ & $1.15173 \mathrm{E}-04$ & 34 & $5.24752 E+04$ & $1.35538 \mathrm{E}-03$ \\
\hline 4 & $8.18730 E+06$ & $1.38942 E-04$ & 35 & $3.43067 E+04$ & $1.56990 \mathrm{E}-03$ \\
\hline 5 & $6.70320 \mathrm{E}+06$ & $1.82032 \mathrm{E}-04$ & 36 & $2.85011 E+04$ & $1.64764 \mathrm{E}-03$ \\
\hline 6 & $5.48811 \mathrm{E}+06$ & $1.88699 \mathrm{E}-04$ & 37 & $2.70001 E+04$ & $1.64764 \mathrm{E}-03$ \\
\hline 7 & $4.49329 E+06$ & $1.69186 \mathrm{E}-04$ & 38 & $2.60584 E+04$ & $1.64810 \mathrm{E}-03$ \\
\hline 8 & $3.67879 E+06$ & $1.53398 \mathrm{E}-04$ & 39 & $2.47875 E+04$ & $1.87795 \mathrm{E}-03$ \\
\hline 9 & $3.01194 \mathrm{E}+06$ & $1.66165 \mathrm{E}-04$ & 40 & $2.35786 \mathrm{E}+04$ & $2.00018 \mathrm{E}-03$ \\
\hline 10 & $2.46597 \mathrm{E}+06$ & $1.94736 \mathrm{E}-04$ & 41 & $1.50344 \mathrm{E}+04$ & $2.71513 \mathrm{E}-03$ \\
\hline 11 & $2.34570 \mathrm{E}+06$ & $1.94736 \mathrm{E}-04$ & 42 & $9.11882 E+03$ & $3.19773 \mathrm{E}-03$ \\
\hline 12 & $2.23130 E+06$ & $1.94743 \mathrm{E}-04$ & 43 & $5.53084 E+03$ & $3.95969 \mathrm{E}-03$ \\
\hline 13 & $2.01897 \mathrm{E}+06$ & $2.41790 \mathrm{E}-04$ & 44 & $3.70744 E+03$ & 4.71797E-03 \\
\hline 14 & $1.65299 E+06$ & $1.70912 \mathrm{E}-04$ & 45 & $3.03539 E+03$ & $5.00935 E-03$ \\
\hline 15 & $1.35335 E+06$ & $1.36184 \mathrm{E}-04$ & 46 & $2.61259 E+03$ & $5.62130 \mathrm{E}-03$ \\
\hline 16 & $1.10803 E+06$ & $1.45472 \mathrm{E}-04$ & 47 & $2.24867 \mathrm{E}+03$ & $5.62257 \mathrm{E}-03$ \\
\hline 17 & $9.07180 E+05$ & $1.76148 \mathrm{E}-04$ & 48 & $2.03468 E+03$ & $6.86570 E-03$ \\
\hline 18 & $7.42735 E+05$ & $2.37194 \mathrm{E}-04$ & 49 & $1.23410 \mathrm{E}+03$ & $9.46083 E-03$ \\
\hline 19 & $6.08101 E+05$ & $3.25077 \mathrm{E}-04$ & 50 & $7.48518 \mathrm{E}+02$ & $1.06452 \mathrm{E}-02$ \\
\hline 20 & $5.23397 \mathrm{E}+05$ & $3.25078 \mathrm{E}-04$ & 51 & $4.53999 E+02$ & $1.55501 E-02$ \\
\hline 21 & $4.97870 \mathrm{E}+05$ & $3.87073 \mathrm{E}-04$ & 52 & $2.75364 \mathrm{E}+02$ & $1.89013 E-02$ \\
\hline 22 & $3.87742 \mathrm{E}+05$ & $4.31607 \mathrm{E}-04$ & 53 & $1.67017 \mathrm{E}+02$ & $2.22467 \mathrm{E}-02$ \\
\hline 23 & $3.01974 \mathrm{E}+05$ & 4.81773E-04 & 54 & $1.01301 \mathrm{E}+02$ & $3.18796 \mathrm{E}-02$ \\
\hline 24 & $2.98491 E+05$ & $4.81773 \mathrm{E}-04$ & 55 & $4.78512 E+01$ & $4.73204 \mathrm{E}-02$ \\
\hline 25 & $2.97211 E+05$ & $4.81773 \mathrm{E}-04$ & 56 & $2.26033 E+01$ & $7.00402 \mathrm{E}-02$ \\
\hline 26 & $2.94518 \mathrm{E}+05$ & 4.81773E-04 & 57 & $1.06770 \mathrm{E}+01$ & $1.01495 \mathrm{E}-01$ \\
\hline 27 & $2.73237 \mathrm{E}+05$ & $6.04295 \mathrm{E}-04$ & 58 & $5.04348 E+00$ & $1.46601 \mathrm{E}-01$ \\
\hline 28 & $2.23708 E+05$ & $6.75630 \mathrm{E}-04$ & 59 & $2.38237 E+\infty 0$ & $2.07679 \mathrm{E}-01$ \\
\hline 29 & $1.83156 \mathrm{E}+05$ & 7.35095E-04 & 60 & $1.12535 E+\infty 0$ & $2.28603 \mathrm{E}-01$ \\
\hline 30 & $1.49956 \mathrm{E}+05$ & $7.97690 \mathrm{E}-04$ & $61^{b}$ & 4.13994E-01 & 0.0 \\
\hline 31 & $1.22773 E+05$ & $8.93156 \mathrm{E}-04$ & & & \\
\hline
\end{tabular}

${ }^{2}$ Read as $1.49183 \times 10^{7}$.

bower energy boundary is $1.0 \times 10^{-5} \mathrm{eV}$. 
Table C9. 61-Group Response Function for the 3-inch Bonner Ball. Units are: (Counts $\cdot \mathrm{cm}^{2} /$ neutron).

\begin{tabular}{|c|c|c|c|c|c|}
\hline Group & $\begin{array}{c}\text { Upper } \\
\text { Energy (eV) }\end{array}$ & Response & Group & $\begin{array}{c}\text { Upper } \\
\text { Energy (eV) }\end{array}$ & Response \\
\hline 1 & $1.49183 \mathrm{E}+07^{\mathrm{a}}$ & $3.44697 \mathrm{E}-03$ & 32 & $8.65168 E+04$ & 2.35597E-01 \\
\hline 2 & $1.22140 \mathrm{E}+07$ & 4.36603E- 03 & 33 & $5.65622 E+04$ & $2.35627 \mathrm{E}-01$ \\
\hline 3 & $1.00000 E+07$ & $5.50542 E-03$ & 34 & $5.24752 E+04$ & $2.67017 \mathrm{E}-01$ \\
\hline 4 & $8.18730 E+06$ & $6.86520 \mathrm{E}-03$ & 35 & $3.43067 E+04$ & $2.86389 \mathrm{E}-01$ \\
\hline 5 & $6.70320 \mathrm{E}+06$ & 9.24655E-03 & 36 & $2.85011 E+04$ & $2.92975 \mathrm{E}-01$ \\
\hline 6 & $5.48811 E+06$ & $1.10363 \mathrm{E}-02$ & 37 & $2.70001 E+04$ & $2.92975 \mathrm{E}-01$ \\
\hline 7 & $4.49329 E+06$ & $1.34927 \mathrm{E}-02$ & 38 & $2.60584 E+04$ & $2.93010 \mathrm{E}-01$ \\
\hline 8 & $3.67879 E+06$ & $1.69485 \mathrm{E}-02$ & 39 & $2.47875 E+04$ & $3.10526 \mathrm{E}-01$ \\
\hline 9 & $3.01194 \bar{E}+06$ & 2.11139E-02 & 40 & $2.35786 \mathrm{E}+04$ & $3.18793 \mathrm{E}-01$ \\
\hline 10 & $2.46597 E+06$ & 2.61091E-02 & 41 & $1.50344 E+04$ & $3.61398 \mathrm{E}-01$ \\
\hline 11 & $2.34570 E+06$ & 2.61091E-02 & 42 & $9.11882 E+03$ & $3.84520 \mathrm{E}-01$ \\
\hline 12 & $2.23130 \mathrm{E}+06$ & $2.61100 \mathrm{E}-02$ & 43 & $5.53084 E+03$ & 4.18671E-01 \\
\hline 13 & $2.01897 \mathrm{E}+06$ & $3.19913 \mathrm{E}-02$ & 44 & $3.70744 E+03$ & 4.47832E-01 \\
\hline 14 & $1.65299 \mathrm{E}+06$ & $3.88334 \mathrm{E}-02$ & 45 & $3.03539 E+03$ & 4.58543E-01 \\
\hline 15 & $1.35335 \mathrm{E}+06$ & $4.67488 \mathrm{E}-02$ & 46 & $2.61259 E+03$ & $4.81026 \mathrm{E}-01$ \\
\hline 16 & $1.10803 E+06$ & $5.58410 \mathrm{E}-02$ & 47 & $2.24867 \mathrm{E}+03$ & $4.81064 \mathrm{E}-01$ \\
\hline 17 & $9.07180 E+05$ & $6.59897 \mathrm{E}-02$ & 48 & $2.03468 E+03$ & $5.17541 \mathrm{E}-01$ \\
\hline 18 & $7.42735 \mathrm{E}+0.5$ & $7.72032 \mathrm{E}-02$ & 49 & $1.23410 \mathrm{E}+03$ & $5.81143 \mathrm{E}-01$ \\
\hline 19 & $6.08101 E+05$ & 8.94339E-02 & 50 & $7.48518 E+02$ & $6.07197 \mathrm{E}-01$ \\
\hline 20 & $5.23397 \mathrm{tE}+05$ & $8.94340 \mathrm{E}-02$ & 51 & $4.53999 \mathrm{E}+02^{\prime}$ & $6.98568 \mathrm{E}-01$ \\
\hline 21 & $4.97870 E+05$ & $1.05238 \mathrm{E}-01$ & 52 & $2.75364 \mathrm{E}+02$ & $7.37472 \mathrm{E}-01$ \\
\hline 22 & $3.87742 \mathrm{E}+05$ & $1.21833 \mathrm{E}-01$ & 53 & $1.67017 \mathrm{E}+02$ & $7.76309 \mathrm{E}-01$ \\
\hline 23 & $3.01974 E+05$ & $1.30279 \mathrm{E}-01$ & 54 & $1.01301 E+02$ & $7.87411 \mathrm{E}-01$ \\
\hline 24 & $2.98491 E+05$ & $1.30279 \mathrm{E}-01$ & 55 & $4.78512 \mathrm{E}+01$ & $8.18826 \mathrm{E}-01$ \\
\hline 25 & $2.97211 E+05$ & $1.30279 \mathrm{E}-01$ & 56 & $2.26033 E+01$ & $9.40486 \mathrm{E}-01$ \\
\hline 26 & $2.94518 E+05$ & $1.30279 \mathrm{E}-01$ & 57 & $1.06770 \mathrm{E}+01$ & $1.04548 E+00$ \\
\hline 27 & $2.73237 \mathrm{E}+05$ & $1.44599 \mathrm{E}-01$ & 58 & $5.04348 E+\infty$ & $1.13840 E+\infty$ \\
\hline 28 & $2.23708 E+05$ & $1.59121 \mathrm{E}-01$ & 59 & $2.38237 E+\infty$ & $1.18443 E+\infty$ \\
\hline 29 & $1.83156 \mathrm{E}+05$ & $1.73593 \mathrm{E}-01$ & 60 & $1.12535 \mathrm{E}+00$ & $8.67016 \mathrm{E}-01$ \\
\hline 30 & $1.49956 \mathrm{E}+05$ & $1.87971 \mathrm{E}-01$ & $61^{b}$ & 4.13994E-01 & 0.0 \\
\hline 31 & $1.22773 E+05$ & 2.07011E-01 & & & \\
\hline
\end{tabular}

${ }^{2}$ Read as $1.49183 \times 10^{7}$.

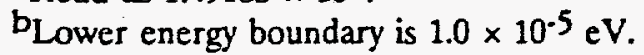


Table C10. 61-Group Response Function for the 5-inch Bonner Ball.

Units are: (Counts $\cdot \mathrm{cm}^{2} /$ neutron).

\begin{tabular}{|c|c|c|c|c|c|}
\hline Group & $\begin{array}{c}\text { Upper } \\
\text { Energy }(\mathrm{cV})\end{array}$ & Response & Group & $\begin{array}{c}\text { Upper } \\
\text { Energy (eV) }\end{array}$ & Response \\
\hline 1 & $1.49183 \mathrm{E}+07^{2}$ & $1.20669 \mathrm{E}-01$ & 32 & $8.65168 E+04$ & $1.24045 \mathrm{E}+\infty$ \\
\hline 2 & $1.22140 \mathrm{E}+07$ & $1.55406 \mathrm{E}-01$ & 33 & $5.65622 E+04$ & $1.24045 E+\infty 0$ \\
\hline 3 & $1.00000 E+07$ & $1.98617 \mathrm{E}-01$ & 34 & $5.24752 E+04$ & $1.23913 E+\infty$ \\
\hline 4 & $8.18730 E+06$ & $2.47835 \mathrm{E}-01$ & 35 & $3.43067 \mathrm{E}+04$ & $1.24014 \mathrm{E}+00$ \\
\hline 5 & $6.70320 E+06$ & $3.09156 \mathrm{E}-01$ & 36 & $2.85011 E+04$ & $1.24050 \mathrm{E}+00$ \\
\hline 6 & $5.48811 \mathrm{E}+06$ & $3.50826 \mathrm{E}-01$ & 37 & $2.70001 E+04$ & $1.24050 E+\infty 0$ \\
\hline 7 & $4.49329 \mathrm{E}+06$ & 4.11506E-01 & 38 & $2.60584 \mathrm{E}+04$ & $1.24051 E+\infty 0$ \\
\hline 8 & $3.67879 E+06$ & 4.80385E-01 & 39 & $2.47875 E+04$ & $1.24352 \mathrm{E}+\infty 0$ \\
\hline 9 & $3.01194 E+06$ & $5.59548 \mathrm{E}-01$ & 40 & $2.35786 \mathrm{E}+04$ & $1.24466 \mathrm{E}+00$ \\
\hline 10 & $2.46597 \mathrm{E}+06$ & $6.44104 \mathrm{E}-01$ & 41 & $1.50344 \mathrm{E}+04$ & $1.25469 \mathrm{E}+\infty$ \\
\hline 11 & $2.34570 \mathrm{E}+06$ & $6.44104 \mathrm{E}-01$ & 42 & $9.11882 E+03$ & $1.26085 \mathrm{E}+\infty 0$ \\
\hline 12 & $2.23130 \mathrm{E}+06$ & $6.44116 \mathrm{E}-01$ & 43 & $5.53084 \mathrm{E}+03$ & $1.26951 \mathrm{E}+00$ \\
\hline 13 & $2.01897 \mathrm{E}+06$ & $7.27626 \mathrm{E}-01$ & 44 & $3.70744 E+03$ & $1.27670 \mathrm{E}+\infty 0$ \\
\hline 14 & $1.65299 \mathrm{E}+06$ & 8.07973E-01 & 45 & $3.03539 E+03$ & $1.27971 \mathrm{E}+\infty 0$ \\
\hline 15 & $1.35335 \mathrm{E}+06$ & $8.84056 \mathrm{E}-01$ & 46 & $2.61259 \mathrm{E}+03$ & $1.28640 \mathrm{E}+\infty 0$ \\
\hline 16 & $1.10803 E+06$ & $9.55019 \mathrm{E}-01$ & 47 & $2.24867 \mathrm{E}+03$ & $1.28641 \mathrm{E}+\infty$ \\
\hline 17 & $9.07180 \mathrm{E}+05$ & $1.01825 E+\infty 0$ & 48 & $2.03468 \mathrm{E}+03$ & $1.29539 E+\infty 0$ \\
\hline 18 & $7.42735 \mathrm{E}+05$ & $1.07318 E+\infty 0$ & 49 & $1.23410 \mathrm{E}+03$ & $1.30011 \mathrm{E}+\infty 0$ \\
\hline 19 & $6.08101 E+05$ & $1.12007 \mathrm{E}+\infty$ & 50 & $7.48518 \mathrm{E}+02$ & $1.29933 \mathrm{E}+00$ \\
\hline 20 & $5.23397 \mathrm{E}+05$ & $1.12007 E+\infty 0$ & 51 & $4.53999 \mathrm{E}+02$ & $1.30253 \mathrm{E}+00$ \\
\hline 21 & $4.97870 \mathrm{E}+05$ & $1.16393 E+\infty$ & 52 & $2.75364 \mathrm{E}+02$ & $1.28078 \mathrm{E}+\infty 0$ \\
\hline 22 & $3.87742 E+05$ & $1.19619 \mathrm{E}+\infty 0$ & 53 & $1.67017 \mathrm{E}+02$ & $1.25907 \mathrm{E}+\infty$ \\
\hline 23 & $3.01974 E+05$ & $1.20946 \mathrm{E}+\infty 0$ & 54 & $1.01301 \mathrm{E}+02$ & $1.22880 \mathrm{E}+\infty 0$ \\
\hline 24 & $2.98491 E+05$ & $1.20946 \mathrm{E}+\infty 0$ & 55 & $4.78512 \mathrm{E}+01$ & $1.23420 E+\infty 0$ \\
\hline 25 & $2.97211 E+05$ & $1.20946 \mathrm{E}+00$ & 56 & $2.26033 \mathrm{E}+01$ & $1.21012 \mathrm{E}+\infty$ \\
\hline 26 & $2.94518 E+05$ & $1.20946 \mathrm{E}+00$ & 57 & $1.06770 \mathrm{E}+01$ & $1.14298 \mathrm{E}+00$ \\
\hline 27 & $2.73237 \mathrm{E}+05$ & $1.22429 \mathrm{E}+\infty 0$ & 58 & $5.04348 \mathrm{E}+\infty 0$ & $1.05082 E+00$ \\
\hline 28 & $2.23708 \mathrm{E}+05$ & $1.23395 \mathrm{E}+\infty 0$ & 59 & $2.38237 E+\infty 0$ & $9.13886 \mathrm{E}-01$ \\
\hline 29 & $1.83156 E+05$ & $1.23969 E+\infty 0$ & 60 & $1.12535 \mathrm{E}+00$ & $5.47689 \mathrm{E}-01$ \\
\hline 30 & $1.49956 \mathrm{E}+05$ & $1.24235 \mathrm{E}+\infty 0$ & $61^{b}$ & 4.13994E-01 & 0.0 \\
\hline 31 & $1.22773 E+05$ & $1.24263 E+\infty 0$ & & & \\
\hline
\end{tabular}

${ }^{2}$ Read as $1.49183 \times 10^{7}$.

bower energy boundary is $1.0 \times 10^{-5} \mathrm{eV}$. 
Table C11. 61-Group Response Function for the 8-inch Bonner Ball.

Units are: (Counts $\cdot \mathrm{cm}^{2} /$ neutron).

\begin{tabular}{|c|c|c|c|c|c|}
\hline Group & $\begin{array}{c}\text { Upper } \\
\text { Energy (eV) }\end{array}$ & Response & Group & $\begin{array}{c}\text { Upper } \\
\text { Energy (eV) }\end{array}$ & Response \\
\hline 1 & $1.49183 E+07^{a}$ & $4.32326 \mathrm{E}-01$ & 32 & $8.65168 E+04$ & $7.23162 \mathrm{E}-01$ \\
\hline 2 & $1.22140 E+07$ & $5.39716 \mathrm{E}-01$ & 33 & $5.65622 E+04$ & $7.23108 \mathrm{E}-01$ \\
\hline 3 & $1.00000 \mathrm{E}+07$ & $6.67276 \mathrm{E}-01$ & 34 & $5.24752 E+04$ & $6.69284 E-01$ \\
\hline 4 & $8.18730 E+06$ & $7.86112 \mathrm{E}-01$ & 35 & $3.4306 \pi \mathrm{E}+04$ & $6.43675 \mathrm{E}-01$ \\
\hline 5 & $6.70320 E+06$ & $9.07761 \mathrm{E}-01$ & 36 & $2.85011 E+04$ & $6.35525 \mathrm{E}-01$ \\
\hline 6 & $5.48811 E+06$ & $9.90151 E-01$ & 37 & $2.70001 \mathrm{E}+04$ & $6.35525 \mathrm{E}-01$ \\
\hline 7 & $4.49329 E+06$ & $1.06950 \mathrm{E}+00$ & 38 & $2.60584 \mathrm{E}+04$ & $6.35489 \mathrm{E}-01$ \\
\hline 8 & $3.67879 E+06$ & $1.14893 \mathrm{E}+\infty$ & 39 & $2.47875 E+04$ & $6.17751 \mathrm{E}-01$ \\
\hline 9 & $3.01194 E+06$ & $1.24834 E+00$ & 40 & $2.35786 \mathrm{E}+04$ & $6.10005 \mathrm{E}-01$ \\
\hline 10 & $2.46597 \mathrm{E}+06$ & $1.33619 \mathrm{E}+\infty 0$ & 41 & $1.50344 E+04$ & $5.78153 \mathrm{E}-01$ \\
\hline 11 & $2.34570 \mathrm{E}+06$ & $1.33619 E+\infty$ & 42 & $9.11882 E+03$ & $5.64949 \mathrm{E}-01$ \\
\hline 12 & $2.23130 \mathrm{E}+06$ & $1.33620 \mathrm{E}+\infty 0$ & 43 & $5.53084 E+03$ & $5.46305 \mathrm{E}-01$ \\
\hline 13 & $2.01897 \mathrm{E}+06$ & $1.39025 \mathrm{E}+00$ & 44 & $3.70744 E+03$ & $5.32917 \mathrm{E}-01$ \\
\hline 14 & $1.65299 \mathrm{E}+06$ & $1.41070 \mathrm{E}+00$ & 45 & $3.03539 E+03$ & $5.28635 \mathrm{E}-01$ \\
\hline 15 & $1.35335 E+06$ & $1.40779 \mathrm{E}+\infty 0$ & 46 & $2.61259 \mathrm{E}+03$ & $5.20655 \mathrm{E}-01$ \\
\hline 16 & $1.10803 E+06$ & $1.38552 \mathrm{E}+00$ & 47 & $2.24867 \mathrm{E}+03$ & $5.20642 E-01$ \\
\hline 17 & $9.07180 E+05$ & $1.34711 E+\infty$ & 48 & $2.03468 E+03$ & $5.08575 \mathrm{E}-01$ \\
\hline 18 & $7.42735 E+05$ & $1.29615 E+\infty 0$ & 49 & $1.23410 E+03$ & $4.85726 \mathrm{E}-01$ \\
\hline 19 & $6.08101 E+05$ & $1.23801 E+\infty 0$ & 50 & $7.48518 \mathrm{E}+02$ & $4.75849 \mathrm{E}-01$ \\
\hline 20 & $5.23397 \mathrm{E}+05$ & $1.23801 E+\infty 0$ & 51 & $4.53999 E+02$ & $4.50639 \mathrm{E}-01$ \\
\hline 21 & $4.97870 E+05$ & $1.16242 \mathrm{E}+\infty$ & 52 & $2.75364 \mathrm{E}+02$ & $4.31711 \mathrm{E}-01$ \\
\hline 22 & $3.87742 \mathrm{E}+05$ & $1.08623 E+00$ & 53 & $1.67017 \mathrm{E}+02$ & 4.12817E-01 \\
\hline 23 & $3.01974 \mathrm{E}+05$ & $1.04936 \mathrm{E}+00$ & 54 & $1.01301 E+02$ & $4.01933 \mathrm{E}-01$ \\
\hline 24 & $2.98491 E+05$ & $1.04936 E+00$ & 55 & $4.78512 E+01$ & 3.85773E-01 \\
\hline 25 & $2.97211 E+05$ & $1.04936 E+00$ & 56 & $2.26033 E+01$ & $3.62747 \mathrm{E}-01$ \\
\hline 26 & $2.94518 E+05$ & $1.04936 \mathrm{E}+\infty 0$ & 57 & $1.06770 \mathrm{E}+01$ & 3.30363E-01 \\
\hline 27 & $2.73237 \mathrm{E}+05$ & 9.89887E-01 & 58 & $5.04348 E+\infty 0$ & $2.94710 \mathrm{E}-01$ \\
\hline 28 & $2.23708 E+05$ & $9.34528 \mathrm{E}-01$ & 59 & $2.38237 \mathrm{E}+00$ & $2.50440 \mathrm{E}-01$ \\
\hline 29 & $1.83156 \mathrm{E}+05$ & $8.84565 \mathrm{E}-01$ & 60 & $1.12535 \mathrm{E}+00$ & $1.47271 \mathrm{E}-01$ \\
\hline 30 & $1.49956 \mathrm{E}+05$ & $8.39646 \mathrm{E}-01$ & $61^{b}$ & 4.13994E-01 & 0.0 \\
\hline 31 & $1.22773 E+05$ & $7.87488 \mathrm{E}-01$ & & & \\
\hline
\end{tabular}

${ }^{2}$ Read as $1.49183 \times 10^{7}$.

b Lower energy boundary is $1.0 \times 10^{.5} \mathrm{eV}$. 
Table C12. 61-Group Response Function for the 10-inch Bonner Ball.

Units are: (Counts $\cdot \mathrm{cm}^{2} /$ neutron).

\begin{tabular}{|c|c|c|c|c|c|}
\hline Group & $\begin{array}{c}\text { Upper } \\
\text { Energy (cV) }\end{array}$ & Response & Group & $\begin{array}{c}\text { Upper Energy } \\
(\mathrm{eV})\end{array}$ & Response \\
\hline 1 & $1.49183 E+07^{2}$ & $5.78909 \mathrm{E}-01$ & 32 & $8.65168 E+04$ & $2.93774 \mathrm{E}-01$ \\
\hline 2 & $1.22140 \mathrm{E}+07$ & $7.00540 \mathrm{E}-01$ & 33 & $5.65622 E+04$ & $2.93744 \mathrm{E}-01$ \\
\hline 3 & $1.00000 E+07$ & $8.39563 \mathrm{E}-01$ & 34 & $5.24752 \mathrm{E}+04$ & $2.64298 \mathrm{E}-01$ \\
\hline 4 & $8.18730 E+06$ & $9.46618 \mathrm{E}-01$ & 35 & $3.4306 \pi \mathrm{E}+04$ & $2.50817 \mathrm{E}-01$ \\
\hline 5 & $6.70320 E+06$ & $1.04564 E+\infty 0$ & 36 & $2.85011 E+04$ & 2.46583E-01 \\
\hline 6 & $5.48811 E+06$ & $1.10765 E+00$ & 37 & $2.70001 \mathrm{E}+04$ & $2.46583 \mathrm{E}-01$ \\
\hline 7 & $4.49329 E+06$ & $1.12520 \mathrm{E}+\infty 0$ & 38 & $2.60584 E+04$ & 2.46564E-01 \\
\hline 8 & $3.67879 E+06$ & $1.14494 E+\infty 0$ & 39 & $2.47875 E+04$ & $2.37427 \mathrm{E}-01$ \\
\hline 9 & $3.01194 \mathrm{E}+06$ & $1.18825 E+\infty 0$ & 40 & $2.35786 \mathrm{E}+04$ & 2.33527E-01 \\
\hline 10 & $2.46597 \mathrm{E}+06$ & $1.21060 \mathrm{E}+00$ & 41 & $1.50344 E+04$ & 2.17585E-01 \\
\hline 11 & $2.34570 E+06$ & $1.21060 \mathrm{E}+\infty 0$ & 42 & $9.11882 E+03$ & $2.11235 E-01$ \\
\hline 12 & $2.23130 E+06$ & $1.21060 E+00$ & 43 & $5.53084 \mathrm{E}+03$ & $2.02107 \mathrm{E}-01$ \\
\hline 13 & $2.01897 \mathrm{E}+06$ & $1.18985 E+00$ & 44 & $3.70744 \mathrm{E}+03$ & $1.95759 \mathrm{E}-01$ \\
\hline 14 & $1.65299 \mathrm{E}+06$ & $1.13338 E+\infty$ & 45 & $3.03539 E+03$ & $1.93728 \mathrm{E}-01$ \\
\hline 15 & $1.35335 \mathrm{E}+06$ & $1.06010 \mathrm{E}+00$ & 46 & $2.61259 E+03$ & $1.89962 \mathrm{E}-01$ \\
\hline 16 & $1.10803 E+06$ & $9.77055 \mathrm{E}-01$ & 47 & $2.24867 \mathrm{E}+03$ & $1.89956 \mathrm{E}-01$ \\
\hline 17 & $9.07180 E+05$ & $8.90321 E-01$ & 48 & $2.03468 E+03$ & $1.84405 \mathrm{E}-01$ \\
\hline 18 & $7.42735 E+05$ & $8.03759 \mathrm{E}-01$ & 49 & $1.23410 \mathrm{E}+03$ & $1.74474 \mathrm{E}-01$ \\
\hline 19 & $6.08101 E+05$ & $7.22208 \mathrm{E}-01$ & 50 & $7.48518 E+02$ & $1.70306 \mathrm{E}-01$ \\
\hline 20 & $5.23397 E+05$ & $7.22208 \mathrm{E}-01$ & 51 & $4.53999 E+02$ & $1.59811 \mathrm{E}-01$ \\
\hline 21 & $4.97870 E+05$ & $6.33772 \mathrm{E}-01$ & 52 & $2.75364 \mathrm{E}+02$ & $1.52531 \mathrm{E}-01$ \\
\hline 22 & $3.87742 E+05$ & $5.56978 \mathrm{E}-01$ & 53 & $1.67017 \mathrm{E}+02$ & $1.45263 \mathrm{E}-01$ \\
\hline 23 & $3.01974 E+05$ & $5.22467 \mathrm{E}-01$ & 54 & $1.01301 E+02$ & $1.53973 \mathrm{E}-01$ \\
\hline 24. & $2.98491 E+05$ & $5.22467 \mathrm{E}-01$ & 55 & $4.78512 E+01$ & $1.47152 \mathrm{E}-01$ \\
\hline 25 & $2.97211 E+05$ & $5.22467 \mathrm{E}-01$ & 56 & $2.26033 E+01$ & $1.37944 \mathrm{E}-01$ \\
\hline 26 & $2.94518 E+05$ & $5.22467 \mathrm{E}-01$ & 57 & $1.06770 \mathrm{E}+01$ & $1.25359 \mathrm{E}-01$ \\
\hline 27 & $2.73237 \mathrm{E}+05$ & $4.72399 \mathrm{E}-01$ & 58 & $5.04348 E+00$ & $1.11704 \mathrm{E}-01$ \\
\hline 28 & $2.23708 E+05$ & 4.29645E-01 & 59 & $2.3823 \pi E+\infty$ & $9.48862 \mathrm{E}-02$ \\
\hline 29 & $1.83156 \mathrm{E}+05$ & $3.93889 \mathrm{E}-01$ & 60 & $1.12535 \mathrm{E}+00$ & $5.57627 \mathrm{E}-02$ \\
\hline 30 & $1.49956 \mathrm{E}+05$ & $3.63828 \mathrm{E}-01$ & $61^{b}$ & 4.13994E-01 & 0.0 \\
\hline 31 & $1.22773 \mathrm{E}+05$ & $3.31231 \mathrm{E}-01$ & & & \\
\hline
\end{tabular}

${ }^{2}$ Read as $1.49183 \times 10^{7}$.

b Lower energy boundary is $1.0 \times 101^{.5} \mathrm{eV}$. 

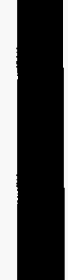


\section{REFERENCES}

1. F. J. Muckenthaler et al., "Measurements for the JASPER Program Radial Shield Attenuation Experiment," ORNL/TM-10371, May 1987.

2. W. A. Rhoades and R. L. Childs, "An Updated Version of the DOT 4 One- and Two-Dimensional Neutron/Photon Transport Code," ORNL-5851, July 1982.

3. W. W. Engle, Jr., "A User's Manual for ANISN: A One-Dimensional Discrete Ordinates Transport Code with Anisotropic Scattering," Union Carbide Nuclear Division Report K-1693, 1967.

4. D. E. Bartine, F. R. Mynatt, and E. M. Oblow, SWANLAKE, "A Computer Code Utilizing ANISN Radiation Transport Calculations for Cross Section Sensitivity Analysis," ORNL/TM-3809, May 1973.

5. R. E. Maerker et al., "Final Report on a Benchmark Experiment for Neutron Transport in Thick Sodium," ORNL-4880, 1974.

6. C. Y. Fu and D. T. Ingersoll, "VELM61 and VELM22: Multigroup Cross-Section Libraries for Sodium-Cooled Reactor Shield Analysis," ORNL/TM-10302, April 1987.

7. C. Y. Fu and D. M. Hetrick, "Update of ENDF/B-V Mod-3 Iron: Neutron-Producing Reaction Cross Sections and Energy-Angle Correlations," ORNLTM-9964 (ENDF-341), July 1986.

8. R. E. Maerker et al., "Response Functions for Bonner Ball Neutron Detectors," ORNLTM-3451, 1971.

9. W. W. Engle, Jr., "AMP (Activity Manipulation Program)," ORNLTM-5296, Union Carbide Corp., Oak Ridge National Lab., June 1975.

10. R. E. Maerker and F. J. Muckenthaler, "The Absolute Neutron Spectrum Emerging Through the Large Beam Collimator from the TSR-II Reactor at the Tower Shielding Facility," ORNL/TM-5183, 1976.

11. C. O. Slater, "Analysis of the ORNL/TSF Alternate Shielding Materials Experiment," ORNL/TM-10183, April 1987.

12. F. J. Muckenthaler et al., "Measurements for the LMR Alternate Shielding Materials Experiment," ORNL/TM-9977, October 1986. 

ORNLTM-10701

Dist. Category UC-535

\section{INTERNAL DISTRIBUTION}
1. B. R. Appleton
2. Y. Y. Azmy
3. J. M. Barnes
4. B. L. Broadhead
5. J. A Bucholz
6. T. J. Burns
7. R. L. Childs
8. J. D. Drischler
9. M. B. Emmett
10. W. W. Engle, Jr.
11. C. Y. Fu
12. L. B. Holland
13. F. J. Homan
14. J. L. Hull
15. H. J. Hunter
16. D. T. Ingersoll
17. J. O. Johnson
18. R. A. Lillie
19. R. E. Maerker
20. F. J. Muckenthaler
21. F. R. Mynatt
22-26. J. V. Pace, III
27. W. A Rhoades
28. R. W. Roussin
29. R. T. Santoro
30. A Shono
31-35. C. O. Slater
36. M. S. Smith
37. R. R. Spencer
38. J. S. Tang
39. R. C. Ward
40. J. D. White
41. J. E. White
42. L. R. Williams
43. EPMD Reports Office
44-45. Laboratory Records Department
46. Laboratory Records, ORNL-RC
47. Document Reference Section
48. Central Research Library
49. ORNL Patent Section

\section{EXTERNAL DISTRIBUTION}

50. Office of the Assistant Manager for Energy Research and Development, Department of Energy, Oak Ridge Operations, P.O. Box 2001, Oak Ridge, TN 37831-6269

51-62. Office of Scientific and Technical Information, P.O. Box 62, Oak Ridge, TN 37831

63. L. F. Blankner, Energy Research and Development, DOE-OR, P.O. Box 2008, Oak Ridge, TN 37831-6269

64. R. W. Brockett, Harvard University, Pierce Hall, 29 Oxford Street, Cambridge, MA 02138

65. L. L. Carter, Westinghouse Hanford Company, 400 Area Trailer 1, P.O. Box 1970, Richland, WA 99352

66. K. Chatani, Power Reactor and Nuclear Fuel Development Corporation, 4002 Narita-Cho, O-Arai-Machi, Ibaraki-Ken, 311-13, Japan

67. R. K. Disney, Westinghouse Electric Company, P.O. Box 158, Madison, PA 15663 
68. J. J. Dorning, Department of Nuclear Enginecring and Engincering Physics, Reactor Facility, University of Virginia, Charlottesville, VA 22903-2442

69. P. B. Hemmig, Safety and Physics Branch, Office of Technology Support Programs, DOE-Washington, Washington, DC 20585

70. J. E. Leiss, Rt. 2, Box 142C, Broadway, VA 22815

71. Professor Neville Moray, University of Illinois, Dept. of Mechanical and Ind. Engineering, 1206 West Green Street, Urbana, IL 61801

72. M. F. Wheeler, Department of Mathematics, Rice University, Post Office Box 1892, Houston, Texas 77251

73. K. Takahashi, Power Reactor and Nuclear Fuel Development Corporation, Sankaido Building, 9-13, 1-Chrome, Minato-Ku, Tokyo, Japan

74. M. Tsutsumi, Power Reactor and Nuclear Fuel Development CorporationWashington, Suite 715, 2600 Virginia Avenue NW, Washington, DC 20037

75-149. Given distribution as shown in DOE/OSTI-4500-R75, LMFBR-Reactor Core Systems, UC.535 